\title{
Swelling and compression of intervertebral disc tissue : model and experiment
}

Citation for published version (APA):

Houben, G. B. (1996). Swelling and compression of intervertebral disc tissue : model and experiment. [Doctoral Thesis, Maastricht University]. Universiteit Maastricht. https://doi.org/10.26481/dis.19961206gh

Document status and date:

Published: 01/01/1996

DOI:

10.26481/dis.19961206gh

Document Version:

Publisher's PDF, also known as Version of record

\section{Please check the document version of this publication:}

- A submitted manuscript is the version of the article upon submission and before peer-review. There can be important differences between the submitted version and the official published version of record.

People interested in the research are advised to contact the author for the final version of the publication, or visit the DOI to the publisher's website.

- The final author version and the galley proof are versions of the publication after peer review.

- The final published version features the final layout of the paper including the volume, issue and page numbers.

Link to publication

\footnotetext{
General rights rights.

- You may freely distribute the URL identifying the publication in the public portal. please follow below link for the End User Agreement:

www.umlib.nl/taverne-license

Take down policy

If you believe that this document breaches copyright please contact us at:

repository@maastrichtuniversity.nl

providing details and we will investigate your claim.
}

Copyright and moral rights for the publications made accessible in the public portal are retained by the authors and/or other copyright owners and it is a condition of accessing publications that users recognise and abide by the legal requirements associated with these

- Users may download and print one copy of any publication from the public portal for the purpose of private study or research.

- You may not further distribute the material or use it for any profit-making activity or commercial gain

If the publication is distributed under the terms of Article $25 \mathrm{fa}$ of the Dutch Copyright Act, indicated by the "Taverne" license above, 


\section{SWELLING AND COMPRESSION OF INTERVERTEBRAL DISC TISSUE \\ -model and experiment-}


Financial support by

Het Nationaal Reumafonds

for the publication of this thesis

is gratefully acknowledged.

Gerard Boudewijn Houben

Swelling and compression of intervertebral disc tissue: model and experiment/

Gerard Boudewijn Houben

Thesis Rijksuniversiteit Maastricht

Vormgeving: Gerard Houben

Druk: Ponsen \& Looijen B.V., Wageningen. Met lit. opg. - Met Nederlandse samenvatting

ISBN 90-9010054-7

Trefwoorden: biomechanics, intervertebral disc 


\section{SWELLING AND COMPRESSION OF INTERVERTEBRAL DISC TISSUE \\ -model and experiment-}

\section{PROEFSCHRIFT}

ter verkrijging van de graad van doctor aan de Universiteit Maastricht,

op gezag van de Rector Magnificus, Prof. mr. M.J. Cohen, volgens het besluit van het College van Decanen,

in het openbaar te verdedigen

op vrijdag 6 december 1996 orn 12.00 uur

door

Gerard Boudewijn Houben

geboren te Zaandam op 19 oktober 1961 
Promotores

Prof. Dr. Ir. J.D. Janssen

Prof. Dr. A. Huson (Technische Universiteit Eindhoven)

Co-promotor

Dr. Ir. J.M.R.J. Huyghe (Technische Universiteit Eindhoven)

Beoordelingscomissie Prof. Dr. H. Kuipers (voorzitter)

Prof. Dr. Ir. M.G.J. Arts

Dr. Ir. P.H.M. Bovendeerd ('Technische Universiteit Eindhoven)

Dr. Ir. C.W.J. Oomens (Technische Universiteit Eindhoven)

Prof. Dr. G.J. van der Vusse 



\section{Contents}

Nomenclature . . . . . . . . . . . . . . . . . . 1

1 General introduction 5

1.1 Introduction . . . . . . . . . . . . . . . . 6

1.2 Anatomy and biochemistry of the intervertebral disc $\ldots \ldots \ldots \ldots$

1.2 .1 Proteoglycans . . . . . . . . . . . . . . 8

1.2 .2 Collagen . . . . . . . . . . . . . . . . . 9

1.2 .3 Non-collagenous proteins . . . . . . . . . . . . . 10

1.2 .4 The nucleus pulposus . . . . . . . . . . . . 10

1.2 .5 The annulus fibrosus . . . . . . . . . . . . 11

1.2 .6 The end plates ........................ 11

1.3 The mechanical functioning of the disc . . . . . . . . . . 12

1.3.1 Poro-viscoelasticity and creep of the motion segment . . . . . 12

1.3.2 Swelling of cartilaginous tissues . . . . . . . . . 15

1.3.3 Tissue deformation; poro-viscoelastic properties of the IVD and IVD annulus . . . . . . . . . . . . . . . . . 25

1.4 Models of IVD and other cartilaginous tissues . . . . . . . . 29

1.4.1 Physico-chemical models . . . . . . . . . . . . . . 29

1.4 .2 Single phase and biphasic continuum models . . . . . . . . 30

1.4 .3 The triphasic model . . . . . . . . . . . . 30

1.4 .4 Finite element models . . . . . . . . . . . . . . 31

1.5 Aims and contents of this thesis . . . . . . . . . . . 33

2 Theory of triphasic mixtures $\quad 35$

2.1 Introduction . . . . . . . . . . . . . . . . . . 36

2.2 General mixture theory . . . . . . . . . . . . . . . 37

2.2 .1 The mass balance . . . . . . . . . . . . . . . 37

2.2 .2 The balance of momentum . . . . . . . . . . . . 38

2.2 .3 The balance of moment of momentum . . . . . . . . . 38

2.2.4 The balance of energy (first axiom of thermodynamics) . . . . . . 38

2.2 .5 The entropy inequality (second axiom of thermodynamics) . . . . 39

2.3 The three component mixture as a model for cartilaginous tissues . . . . 39

2.3.1 Assumptions regarding the three component mixture of cartilaginous tissues .................... . . . 40

2.3.2 Combining the first and second laws of thermodynamics to derive the differential equations for fluid flow and ion diffusion . . . . . . 42 
2.3.3 Constitutive behaviour; the Cauchy stresses, fluid pressure, strain energy function . . . . . . . . . . . 46

2.4 The differential equations based on chemical potentials $\ldots \ldots \ldots 47$

2.4 .1 The degrees of freedom . . . . . . . . . . . . . . 47

2.4 .2 The momentum equation . . . . . . . . . . . . . 49

2.4 .3 The continuity equation $\ldots \ldots \ldots \ldots \ldots$

2.4 The diffusion equation . . . . . . . . . . . . . . 50

2.4.5 Summary of differential equations and boundary conditions . . . . 50

3 A finite element formulation for the triphasic mechanics of cartilaginous materials based on the chemical potentials of fluid and ions $\mathbf{5 3}$

3.1 Introduction . . . . . . . . . . . . . . . . . . . . 54

3.2 The finite element model . . . . . . . . . . . . . . . . . . 55

3.2 .1 Discretization . . . . . . . . . . . . . . . . 58

3.3 The $1 \mathrm{D}$ computer implementation . . . . . . . . . . . . . . 61

3.3.1 Deformation dependencies; constitutive behaviour . . . . . . . . 63

3.4 Comparison of the ID model with analytical solutions . . . . . . . . 65

3.4.1 Linear biphasic compression . . . . . . . . . . . . . 65

3.4 .2 Diffusion . . . . . . . . . . . . . . . . . 67

3.5 The ID computer sirnulation of CSC experiments . . . . . . . 68

3.6 Discussion . . . . . . . . . . . . . . . . . . . . . . 72

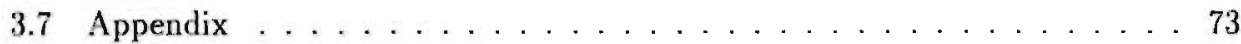

4 Non-homogeneous permeability of canine anulus fibrosus $\quad 75$

4.1 Summary . . . . . . . . . . . . . . . . . 76

4.2 Introduction . . . . . . . . . . . . . . . . 77

4.3 Methods . . . . . . . . . . . . . . . . . . . . . . . . . . 79

4.3 .1 Sample preparation . . . . . . . . . . . . . . . . 79

4.3.2 Confined swelling and compression setup . . . . . . 80

4.3 .3 Experimental protocol . . . . . . . . . . . . . . . . . 81

4.3.4 Data analysis . . . . . . . . . . . . . . 82

4.4 Results. . . . . . . . . . . . . . . . . . . . . . . 86

4.5 Discussion . . . . . . . . . . . . . . . . . . . . 90

4.5.1 Permeability as a function of radial position in the disc, and conditioning phase strain . . . . . . . . . . . . 90

4.5.2 Approximation of the permeability coefficient . . . . . . . 91

4.5 .3 The aggregate modulus . . . . . . . . . . . . 92

4.5 .4 In vitro versus in vivo state $\ldots \ldots \ldots \ldots \ldots \ldots \ldots \ldots$

4.5.5 Leaching of proteoglycans . . . . . . . . . . . . . 993

4.5 .6 Conclusions . . . . . . . . . . . . . . . . . 94

5 Triphasic material parameters of canine anulus fibrosus 95

5.1 Surnmary . . . . . . . . . . . . . . . . . . . 96

5.2 Introduction . . . . . . . . . . . . . . . . . . 98

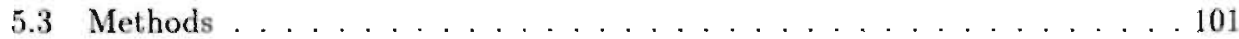

5.3 .1 Sample preparation . . . . . . . . . . . . 101 
5.3.2 Confined swelling and compression (CSC) setup . . . . . . . . . 102

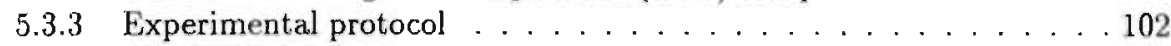

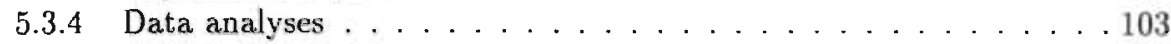

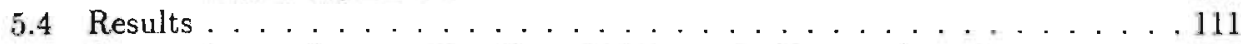

5.4.1 Parameters as a function of distance to the annulus edge . . . . . 111

5.4 .2 The mean activity and osmotic coefficients . . . . . . . . . . . 114

5.4 .3 The intrafibrillar water factor: $\phi_{c i} \ldots \ldots \ldots . \ldots . \ldots 114$

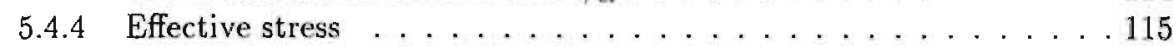

5.4.5 The 1D triphasic computer fits of the CSC experiments . . . . 116

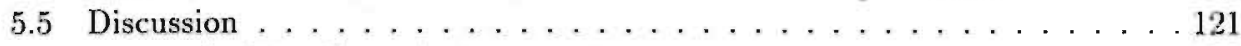

5.5.1 Position dependent parameters . . . . . . . . . . . . . . 122

5.5.2 Effective stress-strain law and stress free state of the sample . . . . 125

5.5.3 The influence of $\phi_{c i}$ on calculated results . . . . . . . . . . . 125

5.5.4 The 1D triphasic computer fits of the CSC experiments . . . . . 126

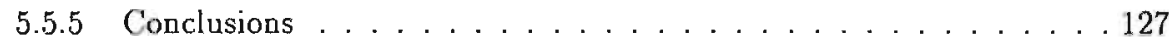

6 General discussion, conclusions and recommendations 129

6.1 Introduction . . . . . . . . . . . . . . . . . . . . . . . . . . . . . . . . . . .

6.2 The determination of chemo-mechanical quantities of the IVD AF . . . . 130

6.2.1 Fitting the equilibrium situations of the CSC experiments using Donnan osmosis and introducing extra- and intrafibrillar fluid com-

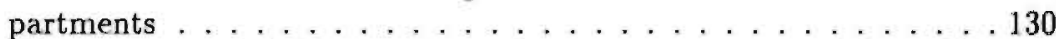

6.2.2 The computer fit of the swelling and compression stages of the CSC experiments with a single fluid compartment . . . . . . . . 132

6.2.3 The CSC experiment as an instrument to determine chemo-mechanical

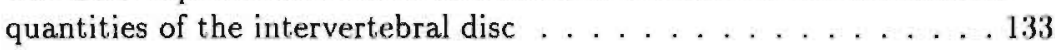

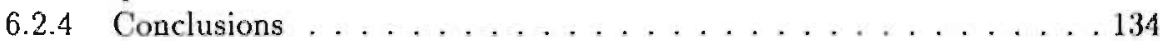

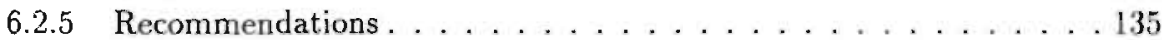

6.3 Dependence on radial position of chemo-mechanical parameters and properties of the annulus fibrosus of the canine intervertebral disc . . . . . . 136

6.3.1 Fixed charge density, hydroxyproline, and fluid fraction . . . . . . 136

6.3 .2 Permeability coefficient . . . . . . . . . . . . . . . . . . . . . . . . . . . . .

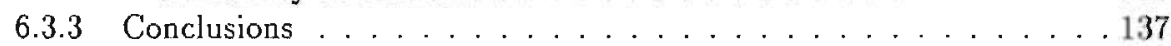

6.4 Relevance of this study and future use . . . . . . . . . . . 138

References

$\begin{array}{ll}\text { Summary } & 156\end{array}$

$\begin{array}{lr}\text { Samenvatting } & 159\end{array}$

$\begin{array}{lr}\text { Nawoord } & 162\end{array}$

$\begin{array}{lr}\text { Curriculum Vitae } & 164\end{array}$ 



\section{Nomenclature}

\section{Symbols}

Symbol SI unit

$\begin{array}{ll}A & \mathrm{~m}^{2} \\ A & \mathrm{~s}^{-1} \\ C & - \\ c & \mathrm{~mol} \cdot \mathrm{m}^{-3} \\ c^{\alpha} & \mathrm{mol} \cdot \mathrm{m}^{-3} \\ c_{ \pm}^{f} & \mathrm{~mol} \cdot \mathrm{m}^{-3} \\ c^{p g} & \mathrm{~mol} \cdot \mathrm{m}^{-3}\end{array}$

$\hat{c}^{\alpha}$

$\mathrm{kg} \cdot \mathrm{m}^{-3} \cdot \mathrm{s}^{-1}$

$c^{\kappa} \quad \mathrm{mol} \cdot \mathrm{m}^{-3}$

$D, D \quad \mathrm{~m}^{3} \cdot \mathrm{s} \cdot \mathrm{kg}^{-1}$

$D^{l} \quad \mathrm{~m}^{2} \cdot \mathrm{s}^{-1}$

$D_{t r i} \quad \mathrm{~m}^{2} \cdot \mathrm{s}^{-1}$

$D^{\alpha} \quad s^{-1}$

d $\mathrm{m}$

$\hat{e}^{\alpha} \quad \mathrm{J} \cdot \mathrm{m}^{-3} \cdot \mathrm{s}^{-1}$

$E^{s}, E^{s}$

$\begin{array}{ll}F & \mathrm{~J}\end{array}$

$F, F$

$F / A \quad \mathrm{~Pa}$

$G \quad$ J

$H_{A} \quad \mathrm{~Pa}$

$H_{e} \quad \mathrm{~Pa}$

hyd $\quad \mathrm{kg} \cdot \mathrm{kg}^{-1}$

hyp

$\boldsymbol{h}^{\alpha}$

J

$K, \boldsymbol{K} \quad \mathrm{m}^{2} \cdot \mathrm{s}^{-1}$

$K_{b i} \quad \mathrm{~m}^{4} \cdot \mathrm{N}^{-1} \cdot \mathrm{s}^{-1}$

$K_{t r i} \quad \mathrm{~m}^{4} \cdot \mathrm{N}^{-1} \cdot \mathrm{s}^{-1}$

$\mathrm{M} \quad \mathrm{mol} \cdot \mathrm{1}^{-1}$

$M^{\text {dif }} \quad \mathrm{kg} \cdot \mathrm{mnol}^{-1}$

$M^{\alpha} \quad \mathrm{kg} \cdot \mathrm{mol}^{-1}$

$M^{ \pm} \quad \mathrm{kg} \cdot \mathrm{mol}^{-1}$ meaning

surface, area

integration constant for Houbolt time integration dimensionless concentration

concentration of external salt per unit fluid volume concentration per unit fluid volume of component $\alpha$ $=\frac{2 v^{\prime}}{\gamma^{\ddagger}}$, variable in numerical formulation concentration per unit fluid volume of the fixed charges of the proteoglycans in an equivalent of moles (moleq) of a uni-valent ion per unit of fluid volume mass of other mixture components converted to mass of component $n$ per unit of volume and time $=\sqrt{\left(c^{p g}\right)^{2}+\left(c_{ \pm}^{f}\right)^{2} \epsilon_{c}}$, variable in numerical formulation diffusion coefficient/tensor in the numerical formulation general diffusion coefficient

triphasic diffusion coefficient derived from $D$

$=\frac{1}{2}\left[\left(\Delta \boldsymbol{v}^{\alpha}\right)^{c}+\left(\Delta \boldsymbol{v}^{\alpha}\right)\right]$, deformation rate tensor of component $\alpha$ distance from the middle of an annulus sample to the edge of the annulus

heat supply per unit of volume and time for component $\alpha$ by other components

Green-Lagrange strain (tensor) of the solid $=F^{s}+F^{\prime}+F^{ \pm}$, the Helmholz free energy of the mixture Helmholz free energy for component $\alpha$ deformation gradient (tensor) externally applied mechanical pressure

Gibbs free energy

compressive aggregate modulus

effective stiffness

hydration in mass of $\mathrm{H}_{2} \mathrm{O}$ per mass of dry weight

percentage of hydroxyproline

partial heat flux vector for component $\alpha$

$=\operatorname{det}(\boldsymbol{F})=d V / d V_{0}$, volume change quotient, elongation factor permeability coefficient/tensor in the numerical formulation biphasic permeability coefficient triphasic permeability coefficient derived from $K$

(without superscript) molar concentration per unit of fluid volume

$=M^{+}-M^{-}$, molar mass of cations minus that of anions

(with superscript) molar mass of component $\alpha$

$=M^{+}+M^{-}$, molar mass of cations plus that of anions 


\begin{tabular}{|c|c|}
\hline$m$ & $\mathrm{~kg}$ \\
\hline$\hat{m}^{\alpha}$ & $\mathrm{kg} \cdot \mathrm{m}^{-1} \cdot \mathrm{s}^{\cdots-2}$ \\
\hline $\mathrm{P}$ & - \\
\hline$p$ & $P_{n}$ \\
\hline $\mathrm{P}_{a}$ & - \\
\hline$\hat{\boldsymbol{p}}^{\alpha}$ & $\mathrm{kg} \cdot \mathrm{m}^{-2} \cdot \mathrm{s}^{-2}$ \\
\hline$q^{\alpha}$ & $m \cdot s^{-2}$ \\
\hline$R$ & $\mathrm{~J} \cdot \mathrm{K}^{-1} \cdot \mathrm{mol}^{-1}$ \\
\hline$\tilde{r}^{\alpha}$ & $\mathrm{J} \cdot \mathrm{kg}^{-1} \cdot \mathrm{s}^{-1}$ \\
\hline$S$ & $J \cdot K^{-1}$ \\
\hline$S, S$ & $\mathrm{~Pa}$ \\
\hline$\tilde{S}^{\alpha}$ & $\mathrm{J} \cdot \mathrm{kg}^{-1} \cdot \mathrm{K}^{-1}$ \\
\hline$T$ & - \\
\hline$T$ & $\mathrm{~K}$ \\
\hline$t$ & $s$ \\
\hline$\tilde{U}^{\alpha}$ & $\mathrm{J} \cdot \mathrm{kg}^{-1}$ \\
\hline$u$ & $\mathrm{~m}$ \\
\hline$V$ & $\mathrm{~m}^{3}$ \\
\hline$V^{\alpha}$ & $\mathrm{m}^{3}$ \\
\hline $\bar{V}^{\alpha}$ & $\mathrm{m}^{3} \cdot \mathrm{mul}^{-1}$ \\
\hline$v, \boldsymbol{v}$ & $\mathrm{m} \cdot \mathrm{s}^{-1}$ \\
\hline $\boldsymbol{w}^{\alpha}$ & $\mathrm{m} \cdot \mathrm{s}^{-1}$ \\
\hline$X$ & - \\
\hline$x$ & - \\
\hline$x, \boldsymbol{x}$ & $\mathrm{m}$ \\
\hline$x_{0}$ & $\mathrm{~m}$ \\
\hline$\beta$ & $\mathrm{kg} \cdot 111$ \\
\hline$\nabla$ & $m^{-1}$ \\
\hline$\nabla_{0}$ & $111^{-1}$ \\
\hline$\epsilon_{\mathrm{c}}$ & - \\
\hline$\epsilon_{p}$ & $=$ \\
\hline$\Phi$ & - \\
\hline$\Phi^{\prime}$ & - \\
\hline$\phi^{\alpha}$ & - \\
\hline$\gamma$ & $\mathrm{kg} \cdot \mathrm{Inol}^{-1}$ \\
\hline$\gamma^{\alpha}$ & - \\
\hline$\gamma^{ \pm}$ & - \\
\hline$\xi$ & - \\
\hline
\end{tabular}

mass

moment of momentum supplied per unit of volume and time by other components to component $\alpha$ probability of rejection of hypothesis hydrostatic fluid pressure probability of rejection of the hypothesis that the slope of a line does not equal zero momentum, supplied by other components to component $\alpha$ per unit of volume and time

body force per unit mass for component $\alpha$ $=8.31441 \mathrm{~J} /(\mathrm{K} \cdot \mathrm{mol})$, molar gas constant

partial specific external heat supply per unit of time for component $\alpha$

entropy

effective second Piola-Kirchhoff stress of the mixture specific entropy of component $\alpha$ dimensionless time absolute temperature time partial specific internal energy of component $\alpha$ $=\boldsymbol{x}-\boldsymbol{x}_{0}$, displacement vector in mixture volume volume taken by component $\alpha$ in the mixture partial molar volume of component $\alpha$ in the mixture velocity (vector)

velocity vector for component $\alpha$ relative to the mixture velocit dimensionless position

molar fraction

position (vector)

reference position vector in mixture

$=\frac{M^{ \pm}\left(c_{t}^{f}\right)^{2}}{4 c^{\kappa}}$, variable in numerical formulation

Eulerian gradient operator

$=\boldsymbol{F}^{c} \cdot \boldsymbol{\nabla}$, Lagrangian gradient operator

$=e^{\frac{\hat{\mu}^{ \pm}-\tilde{\mu}_{0}^{ \pm}}{R T}} M^{ \pm}$, variable in numerical formulation

$=e^{\frac{n^{\prime}-\hat{\mu}_{0}^{\prime}}{R T} M^{\prime}}$, variable in numerical formulation

osmotic coefficient

$=1+\frac{\partial \ln \gamma^{f}}{\partial \ln x^{f}}$, coefficient for variation of $\gamma^{f}$ with $x^{f}$ volume fraction of component $\alpha$

$=\frac{1}{2}\left(M^{\text {dif }}+\frac{M^{ \pm} c^{p s}}{c^{\kappa}}\right)$, variable in numerical formulation

(without superscript)

activity coefficient of component $\alpha$

$=\sqrt{\gamma^{+} \gamma^{-}}$, (average) activity coefficient of the ions

$=\frac{1}{2}\left(M^{d i f} c^{p g}+M^{ \pm} c^{\kappa}\right) \frac{1}{M^{ \pm} c^{\prime}}$, variable in numerical formulation 


$\begin{array}{lll}\xi^{\alpha} & \mathrm{J} \cdot \mathrm{m}^{-3} \cdot \mathrm{s}^{-1} \cdot \mathrm{K}^{-1} & \begin{array}{l}\text { entropy production per unit of volume } \\ \text { and time from component } \alpha\end{array} \\ \tilde{\mu}^{\alpha}, \tilde{M}^{\alpha} & \mathrm{J} \cdot \mathrm{kg}^{-1} & \begin{array}{l}\text { specific chemical potential (tensor) for component } \alpha \\ \pi\end{array} \\ \psi & \mathrm{Pa} & \text { osmotic pressure } \\ \psi & \mathrm{J} \cdot \mathrm{m}^{-3} & \text { strain energy function } \\ \rho^{\alpha} & \mathrm{kg} \cdot \mathrm{m}^{-3} & \text { mass density of component } \alpha \text { on mixture volume basis } \\ \rho_{t}^{\alpha} & \mathrm{kg} \cdot \mathrm{m}^{-3} & \text { true mass density of component } \alpha \text { on component volume basis } \\ \sigma_{e}, \sigma_{e} & \mathrm{~Pa} & \text { effective Cauchy stress (tensor) of the mixture } \\ \boldsymbol{\sigma}^{\alpha} & \mathrm{Pa} & \text { partial Cauchy stress tensor for component } \alpha \\ \tau & \mathrm{S} & \text { time constant }\end{array}$

\section{Abbreviations}

$\mathrm{AF}$

CSC

EP

$\mathrm{FC}$

FCD

$\mathrm{FE}(\mathrm{M})$

GAG

IVD

NP

$\mathrm{PG}$

dof

\section{Subscripts}

0

bi

c.

d

$d w$

e

exf

$\mathrm{h}$

inf

I

$\mathrm{s}$

$\mathrm{s}(\mathbf{w})$

sf

t.

tot

tri

$\mathrm{v}$

ww annulus fibrosus

confined swelling and compression

end plates

fixed (negative) charges

fixed charge density

finite element (model)

glycosaminoglycan

intervertebral disc

nucleus pulposus

proteoglycan(s)

degree of freedom

reference state, or conditioning stage

biphasic

compression stage

diffusion

dry weight

effective (for stress), or element (in FEM volume integrals)

(based on) extrafibrillar (volume)

high

(based on) intratibrillar (volume)

low

solid (only used in time differentiation)

swelling stage

stress free

true

(based on) total (volume)

triphasic

vivo

wet weight 


\section{Superscripts}

$\begin{array}{ll}+ & \text { pusitive ions (cations) } \\ - & \text { negative ions (anions) } \\ \pm & \text { salt molecules (ions), i.e., cations plus anions } \\ \text { dif } & \text { difference of molar masses of cations minus anions } \\ \mathrm{f} & \text { fluid } \\ \mathrm{l} & \text { standard (literature) definition, used on } D \\ \mathrm{~m} & \text { mixture } \\ \mathrm{pg} & \text { fixed charges of proteoglycans } \\ \mathrm{s} & \text { solid } \\ \alpha & \text { any of the components of a mixture } \\ \beta & \text { any of the components fluid or ions }\end{array}$

\section{Notation}

$:=$

$A, a$

$\boldsymbol{a}($ except $\sigma)$

$\|\boldsymbol{a}\|$

$\boldsymbol{A}, \boldsymbol{\sigma}$

I

$A^{*}$

$\tilde{A}$

$\ddot{A}$

$\hat{A}^{\alpha}$

$\bar{A}$

$\check{A}$

$A^{-1}$

$A^{e}$

$\operatorname{det}(\boldsymbol{A})$

$\operatorname{tr}(\boldsymbol{A}):=\boldsymbol{A}: \boldsymbol{I}$

$a b$

$a \cdot b$

$\boldsymbol{A} \cdot \boldsymbol{b}$

$\boldsymbol{A} \cdot \boldsymbol{B}$

$\boldsymbol{A}: \boldsymbol{B}:=\operatorname{tr}(\boldsymbol{A} \cdot \boldsymbol{B})$

time derivatives:

$\frac{\partial a^{\alpha}}{\partial t}$

$a^{\alpha}=\frac{D a^{\alpha}}{D t}=\frac{\partial a^{\alpha}}{\partial t}+v^{m} \cdot \nabla a^{\alpha}$

$\grave{a}^{\alpha}=\frac{D^{\alpha} a^{\alpha}}{\overline{D t}}=\frac{\partial a^{\alpha}}{\partial t}+v^{\alpha} \cdot \nabla a^{\alpha}$

equals by definition

scalar

vector

length of $a$

second order tensor

second order unit tensor

the asterix indicates a Lagrangian quantity

the tilde indicates a specific variable, i.e., per unit mass

the check indicates a volumetric variable, i.e., per unit volume

the hat indicates supply of quantity $A$ from other

components to component $\alpha$

the bar indicates a partial molar quantity

the breve indicates an estimate of $A$

inverse of $\boldsymbol{A}$

conjugate of $\boldsymbol{A}$

determinant of $\boldsymbol{A}$

trace $(\boldsymbol{A})$

diadic product of vectors $\boldsymbol{a}$ and $\boldsymbol{b}$

dot product of vectors $\boldsymbol{a}$ and $\boldsymbol{b}$

dot product of tensor $\boldsymbol{A}$ and vector $\boldsymbol{b}$

dot product of tensors $\boldsymbol{A}$ and $\boldsymbol{B}$

double dot product of tensors $\boldsymbol{A}$ and $\boldsymbol{B}$

variation of $a^{\alpha}$ in time observed fixed in space. (local time derivative)

variation of $a^{\alpha}$ in time, observed fixed to average mixtu motion

variation of $a^{\alpha}$ in time, observed fixed to component $\alpha$

$\left(a^{\alpha}\right)_{\zeta}=\frac{D^{\zeta} a^{\alpha}}{D t}=\frac{\partial a^{\alpha}}{\partial t}+v^{\zeta} \cdot \nabla a^{\alpha}$ variation of $a^{\alpha}$ in time, observed fixed to component $\zeta$ 
Chapter 1

General introduction 


\subsection{Introduction}

Have you ever experienced low back pain? The chance that you can answer this question positively is around $50 \%$. In an epidemiologic study on low back pain of a Dutch population (3091 men and 3493 women 20 years of age and older; study conducted between 1975 and 1978) Valkenburg and Haanen ${ }^{226}$ found that $51 \%$ of the men and $57 \%$ of the women said they had experienced low back pain. Low back pain at the time of examination occurred in $22 \%$ of the adult men and in $30 \%$ of the adult women. For a British survey ${ }^{114}$ these figures were $11 \%$ and $19 \%$, respectively. Of the Dutch survey, $58 \%$ of those men and women ever suffering from low back pain, suffered from it more than once in their lives. Over $30 \%$ had symptoms for more than 3 months, and a similar percentage stayed in bed because of it. Nearly half of the men and one third of the women reported that they had been unfit to work because of low back pain, and $8 \%$ of the men and $4 \%$ of the women had to change jobs as a result of it.

In the United States studies have indicated that impairments of the back and spine are the chronic conditions most frequently causing limitation of activity among persons under age 45 years. Back and spine conditions ranked third in limitation of activity after heart disease, and arthritis and rheumatism in persons 45 to 64 years of age (1969-70). ${ }^{37}$

The impact of low back pain especially on industry is large. In the state of Washington, figures from insurance claims indicated that industrial back injuries accounted for $24 \%$ of days lost from work (1968). ${ }^{137}$ Vermeer ${ }^{229}$ estimated the low back pain related costs in the Netherlands in 1983 about 1 billion US dollars.

The etiology of low back pain is often unclear. There is no consensus on the proportion of all cases of low back pain that is idiopathic (of unknown cause), or on the amount of disability and activity limitation that is attributable specifically to idiopathic low back pain. ${ }^{100}$ The connection between intervertebral disc disease and low back pain is also in many cases obscure. ${ }^{23,142,176,209}$ Psychological factors may play a role in the etiology of low back pain. ${ }^{36}$ It is, however, clear from published accounts that the intervertebral discs exhibit a wide range of microscopic and macroscopic features, which, in some cases, can be associated with specific diseases and syndromes. An example of this is disc herniation. $7,39,43,78,90,153,165$ In disc herniation the fibres of the annulus are ruptured, allowing the gelly-like nucleus to be pushed outwards through the fissure. In the case of a posterior hernia, expression of nucleus material may cause compression of the nerve root, causing low back pain radiating into the lower limb. A model that incorporates the mechanical properties of the solid fibres, and can calculate fibre stresses and strains, may be used to predict the circumstances under which failure of these fibres is most likely to occur. Such a model could also be used to simulate the effects of various changes in the disc's tissue structure, e.g., due to aging, or surgical treatment.

In order to understand the factors that play a role in the etiology of low back pain, the use of mechanical models may thus be helpful. Macromodels give insight in the mechanical functioning of the spine as a whole. Micromodels, that study the intervertebral disc, or even parts of the disc, shed light on the mechanisms that play a role when, e.g., annular ruptures (disc herniation), or change of properties during aging are involved. The development and assessment of this kind of model is the topic of this thesis. 


\subsection{Anatomy and biochemistry of the intervertebral disc}

The human spine is the basic load carrying structure of the upper body. Its structural components make possible a wide range of postures. The spine is build up out of vertebrae, ligaments, and 23 intervertebral discs (IVD).

The vertebrae are bony segments with a central porous cancellous part, and a compact stiff outer cortical shell, that form the vertebral body. Posteriorly, the vertebra has an arc

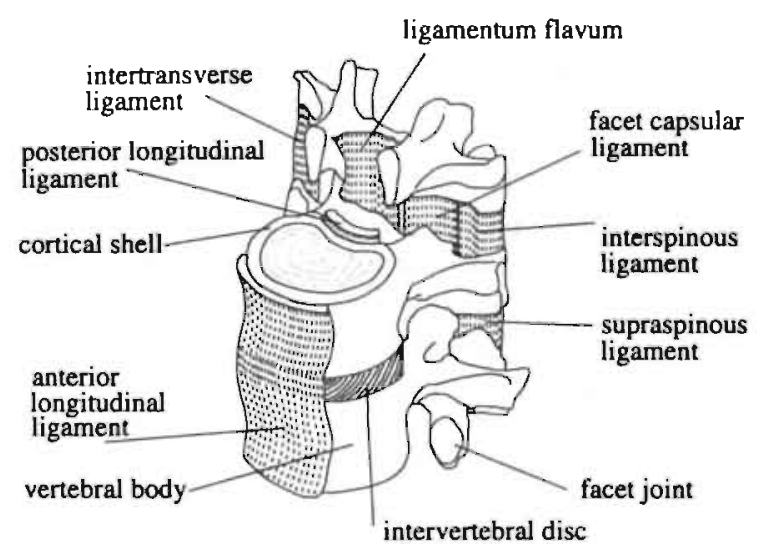

Figure 1.1: Schematic drawing of a lumbar motion segment: the intervertebral disc can be discerned between the vertebral bodies which are connected by the anterior longitudinal ligament. In order to show all the ligaments, a third posterior arc has been added (after White and Panjabi ${ }^{234}$ ).

cervical (C) discs.

The IVD is a largely avascular structure, ${ }^{46,47}$ consisting of a central gelatinous part, the nucleus pulposus (NP), surrounded by the fibrous annulus fibrosus (AF) that is organized

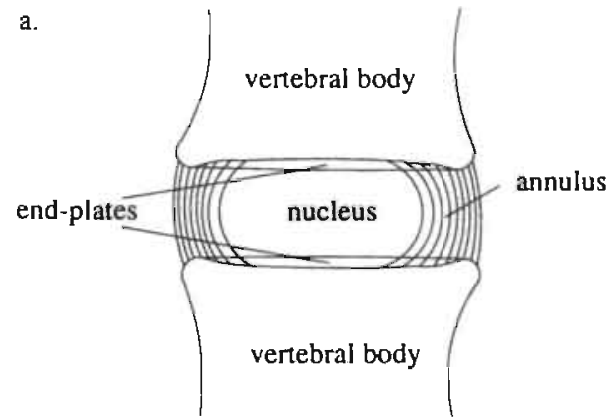

b.

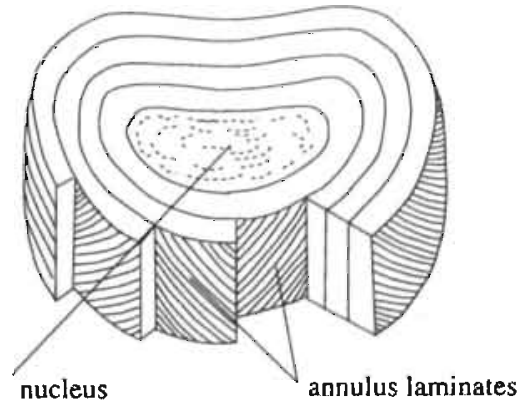

Figure 1.2: Schematic view of a sagital cross-section of a part of the motion segment: vertebral bodies and intervertebral disc (a), and (b) the intervertebral disc's laminate structure of the annulus fibrosus. Part of the annulus is removed to show the laminate structure (after White and Panjabi ${ }^{234}$ ). 
in lamellae, and two end plates (EP) that cover top and bottom of the IVD (Figure 1.2). The IVD consists of three tissue types: chordal tissue (the center of the NP), cartilaginous tissue (transitional zone from NP to AF, inner AF), and connective tissue (outer AF). Although the disc is a structure of three tissue types, it is often referred to as a (cartilaginous) tissue, a habit that will be followed throughout this thesis.

The disc is roughly cylindrical, with a diameter of a few $\mathrm{cm}$, and thickness of around $1 \mathrm{~cm}^{84}$ Disc shape and size vary from caudal to cranial, with the biggest discs in the lumbar region and the most rounded ones in the cervical region. A more detailed description of anatomy, function, structure, and chemical composition is given in Ghosh. ${ }^{62}$ All reference to the IVD will be, unless otherwise mentioned, to the non-degenerate adult human lumbar disc. An exception to this rule is made whenever cited properties are the same or comparable for human and non-human (mostly canine) discs. The main chemical constituents of the disc are water, proteoglycans (PG), collagen, non-collagenous proteins, and small solutes as $\mathrm{NaCl}^{16,22,31,40,58,183,199,237}$ The IVD's chemical composition depends on level and region of the disc, ${ }^{13,17,26,64,89,159,181,199,225}$ age of the animal, ${ }^{6,15}, 26,32,41,76,82,104,141,160,161,185,232$ and pathological condition of the disc. $^{20,21,91,101,117,118,140,156,157,162,180,182,227}$ The IVD have a mean cell density of 5800 cells $/ \mathrm{mm}^{3}$. This number is low compared with articular cartilage $\left(14,000\right.$ cells $\left./ \mathrm{mm}^{3}\right){ }^{132}$ Most of its volume is taken up by the extracellular space, which consists of all the chemical constituents mentioned above. The words "matrix", or "solid matrix" are used by some authors to indicate the proteoglycans and collagen fibres, by others to indicate the PG and water ("proteoglycan matrix" or "proteoglycan gel"). In this thesis we define the matrix, or solid matrix, as the combination of $P G$ and collagen fibres. The solid is defined as all tissue constituents excluding the water and small solutes, such as the mobile ions.

IVD tissue is a cartilaginous tissue as it contains the same components as cartilage, and parallels cartilage in aspects of its mechanical behaviour. Cartilaginous tissues are also referred to as soft, charged tissues, or as porous, fibre reinforced materials.

\subsubsection{Proteoglycans}

A proteoglycan molecule consists of sulphated glycosaminoglycan (GAG) side chains (chondroitin 4- and 6-sulphate (CS) and keratan sulfate (KS)) covalently bound to a protein core (Figure 1.3). GAGs are heteropolysaccharides composed of repeating disaccharide groups in which an $\mathrm{N}$-acetylated hexosamine is linked by a glycosidic bond to a nitrogen-free monosaccharide, usually a uronic acid. A distinctive feature of glycosaminoglycan chains is their high number of fixed charges. The concentration of these fixed charges is called the fixed charge density (FCD). The total negative charge for keratan sulphate is assumed to be one per disaccharide (one sulphate group; $\mathrm{SO}_{3}^{-}$), and that for chondroitin sulphate is two per disaccharide (one sulphate and one carboxyl group; $\left.\mathrm{COO}^{-}\right) .{ }^{133,134,224}$ These charges are fixed on the PG macromolecules, which in turn form a network entangled in the collagen fibres. Therefore, unlike the small ions like $\mathrm{Na}^{+}, \mathrm{Cl}^{-}$, $\mathrm{K}^{+}$, the charges of the proteoglycans are fixed in the tissue. 
a.

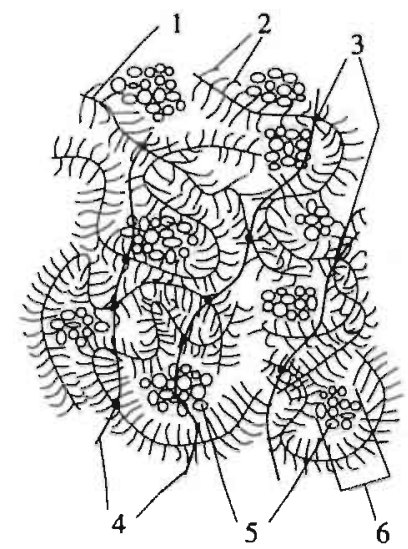

b.

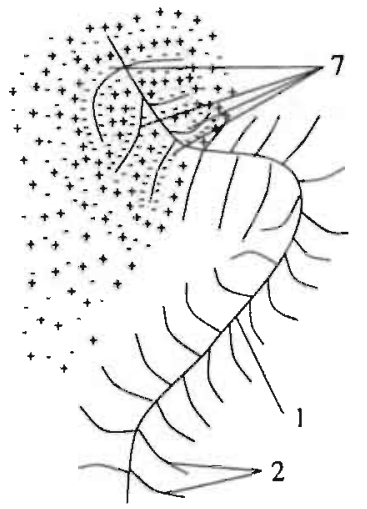

The FCD is an important determinant of the swelling properties (osmotic pressure) of the disc and other cartilaginous tissues. ${ }^{52,53,128,130,135,219,221-224}$

The FCD depends on age and region in the disc, and is about $0.13-0.28 \mathrm{moleq} / \mathrm{kg}$ tissue. ${ }^{219}$ Part of the PG molecules form aggregates as a result of the presence of hyaluronic acid. ${ }^{34,54}$ In these aggregates the protein core is bound to hyaluronic acid by a specific non-covalent interaction which is stabilized by link proteins, and for which ionized carboxyl groups are essential $^{54,173}$ (Figure 1.3a).

Hyaluronic acid is a non-sulphated GAG with a much longer chain length than the sulphated GAGs which form the side-chains of

Figure 1.3: Schematic view of intervertebral disc tissue composition (a), and a proteoglycan molecule (b). In (b) the fixed negative charges on the glycosaminoglycans (enclosed by dotted curves), and the mobile positive and negative charges for part of the structure are schematically shown. 1: protein core of a proteoglycan molecule, 2: glycosaminoglycan side chains, 3: link proteins for aggregation of proteoglycan molecules, 4 : chains of hyaluronic acid molecules, 5: collagen fibrils, 6: collagen fibre (enclosed by a dotted curve), 7: fixed charges on glycosaminoglycans.

the PG.

\subsubsection{Collagen}

The collagen molecule is a protein built of long polypeptide chains of amino acids. The amino acid sequence along each chain defines the primary structure of the collagen. ${ }^{172}$ The chains of amino acids are spatially arranged in a triple helical configuration, forming the molecule. Aggregations of collagen molecules form in most types of collagen various sized fibrils. The fibrils, in their turn, aggregate into the collagen fibre (Figure 1.3a). According to Eyre, eleven genetically distinct types of collagen have been discerned. ${ }^{55}$ These types can be subdivided into 3 classes. One of the distinguishing features of these classes is the length of the molecule. Class 1 contains molecules of a single, $300 \mathrm{~nm}$ long uninterrupted triple helix, class 2 molecules are at least as long as class 1 molecules, but have nontriple-helical domains, and can have globular extension sequences. Class 3 molecules have a much shorter helical domain than class 1 molecules. They have globular extensions at both ends, and the helix may be interrupted along its length. Collagens have charge groups that are mostly ionized at physiological $\mathrm{pH}$, but at $\mathrm{pH} 7.4$ the net charge of the collagen molecules has been shown to be practically zero (slightly positive) because of inter- and intra-molecular electrostatic linkages. ${ }^{115}$

The IVD contains 7 types of collagen: the nucleus contains types II, IV, IX, and XI and the annulus types I, II, III, V, VI, IX, and XI. The most important in the disc are types $I$ and II, both class 1 . Types I and II have opposing concentration gradients in the radial direction of the disc, type I concentration being highest in the outer annulus, decreasing to zero in the transition zone between nucleus and annulus, and type II decreasing from 
a maximum value in the central nucleus to zero in the transition zone.

\subsubsection{Non-collagenous proteins}

The non-collagenous proteins are all proteins other than collagen. These include the PG, glycoproteins, link proteins, elastic fibres, and enzymes. ${ }^{28,63,139}$ The non-collagenous protein content of the disc increases with age and constitutes from $20 \%$ (young discs) to 45 $\%$ (old age) of the dry weight of the nucleus, and from $5 \%$ (young) to $25 \%$ (old) of the dry weight of the annulus. ${ }^{49}$ In these percentages the protein cores of PG subunits may be included (these are already included in the PG content, and thus would be accounted for twice). Despite these high percentages, little is known about the composition and properties of these disc proteins. As the PG have already been discussed above, they will be left out here. Glycoproteins consist of a small number of monosaccharides or branches of oligosaccharides bound to protein. ${ }^{8}$ They bind firmly to collagen fibrils, and form the outer material of elastic fibres. It is believed that they may have an important role in determining the physical properties of collagen fibrils, for example by regulating fibril thickness, water content, and the quality and strength of interaction with PG. ${ }^{54}$ Link protein (also a glycoprotein ${ }^{54}$ ) plays a role in the stabilization of PG aggregates. ${ }^{173}$ Elastic fibres are present in small quantities in both the annulus and the nucleus. ${ }^{29,30,96}$ In the annulus the elastic fibres' shape is almost uniformly cylindrical and they lie parallel and closely related to the collagen fibres, in an arrangement similar to that found in tendons. ${ }^{28,44}$ Elastic fibres of the nucleus are more irregularly shaped, and their orientation is not correlated to that of the collagen fibrils. Although elastic fibres occupy only about $10 \%$ of the solid material of the annulus, with slightly diminishing content over time (12.6\% at the age of $26,8.2 \%$ at the age of 62$),{ }^{87,88,139}$ they are believed to confer elasticity to the $A F{ }^{75,76,143}$

A further contribution to the pool of non-collagenous proteins comes from the enzymes and other cell proteins. ${ }^{171}$

\subsubsection{The nucleus pulposus}

The gelatinous NP consists mainly of a highly hydrated PG mesh, with some criss-cross oriented loose collagen fibrils. In children and young adults, water accounts for over 80 $\%$ of the wet weight of the nucleus, decreasing to around $70 \%$ after the 5 th decade. ${ }^{66}$ PG contribute around $50 \%$ of the dry weight of the nucleus, and collagen in the young NP (3-19 years) is approximately $25 \%$ by dry weight, ${ }^{54}$ but its percentage can be as low as 6 to $8 \%$ in the very central part of the nucleus. ${ }^{212}$ On wet weight basis, collagen accounts for only about $5 \%$ of the weight of the nucleus. ${ }^{118}$ The nuclear collagen fibrils have a diameter of around $30 \mathrm{~nm}$, and are randomly dispersed and not organized into any more complex structure. ${ }^{86}$ Most of the collagen in the nucleus is of type II. ${ }^{63}$ The composition of the NP varies with age. E.g., the water concentration (by wet weight) and $\mathrm{PG}$ concentration (by dry weight) decrease with age. ${ }^{54,66,225}$ 


\subsubsection{The annulus fibrosus}

The AF differs in structure and composition from the nucleus. The chemical components of the annulus are basically the same as those in the nucleus, but they show small chemical differences. In addition, the AF structural organization differs greatly from that of the nucleus. The annulus $\mathrm{PG}$ tend to aggregate more than those in the nucleus, and thus are more firmly bound within the tissue. ${ }^{31,54}$ Furthermore, the annulus PG have a lower hexosamine ( = an amino sugar) content and a higher protein content than the NP..$^{31,54}$ The annulus contains approximately $70 \%$ water (by wet weight), 10-20\% PG by dry weight, and $67 \%$ of collagen by dry weight. ${ }^{54}$

The outer nucleus blurs into the inner annulus in the transitional area. There is no clear boundary between the nucleus and annulus. Going from the transitional area outwards the collagen fibrils are ever more circumferentially directed and organized in fibres, which in turn are organized in lamellae (Figure 1.2b). The outer AF has 15-25 circumferential lamellae. ${ }^{124}$ The successive lamellae have fibre directions that alternate from approximately $65^{\circ}$ to $-65^{\circ}$ to the vertical axis of the spine. ${ }^{79,208}$ The lamellae do not form complete rings, but split intricately or merge to interlock with other lamellae. ${ }^{124}$ A number of outer lamellae are anchored in the vertebrae. More inner annular lamellae merge into the cartilage end plate. ${ }^{85}$ There is a steady increase in the proportion of collagen and a decrease of PG content from the inner to the outer annulus, ${ }^{5,54,118}$ although for collagen content this has not always been confirmed. ${ }^{199}$ Water content decreases from inner to outer annulus. ${ }^{17,199,219}$ The collagen type also varies in radial direction. Inner annulus (transition zone to nucleus) contains predominantly type II (which predorninates in hyaline cartilage), outer annulus contains mainly type I (that can be found in tendon and fibrous cartilage). ${ }^{56,57}$ Collagen fibrils in the annular fibres are surrounded by a layer of hydrated proteoglycan gel. ${ }^{84}$ With increasing age, the annulus fibrils increase in diameter. ${ }^{76}$ There is no evidence of decrease of water content, or PG content. ${ }^{54}$

\subsubsection{The end plates}

The end plates close off the IVD top and bottom. They connect to the vertebrae that enclose the IVD in a layer of calcified material. ${ }^{95}$ On the side facing the nucleus and annulus they are directly connected to the lamellae which form the inner one third of the annulus. ${ }^{10,86}$ The end plates are composed of hyaline cartilage. This type of cartilage lacks the fibrous appearance of annulus, and is more translucent. The orientation of the collagen fibrils is generally parallel to the surface of the end plate. The thickness of the end plate has a mean value of $0.6 \pm 0.3 \mathrm{~mm} .{ }^{183}$ Generally the end plate is thinnest over the nucleus. The end plate contains approximately $55 \%$ of water by wet weight, and $62 \%$ collagen by dry weight. ${ }^{183}$ These values are about the same for both end plate regions over the nucleus and the annulus. PG content is reported by Roberts et al. ${ }^{183}$ as an amount of $\mathrm{D}$ (i)M(ethylmethylene) $\mathrm{B}$ (lue) reactive material. They found for the end plate over the nucleus a DMB content of $36 \%$ of the average nucleus DMB content, and for the end plate over the annulus a DMB content of $48 \%$ of the average annulus DMB content. 


\subsection{The mechanical functioning of the disc}

The primary mechanical function of the vertebral column is to support the upper body and maintain its stability, while being flexible at the same time. The specific structure of the vertebral column makes possible a wide variety of movements and postures. Stability is achieved by the presence of the ligaments, muscles, and vertebrae with their facet joints, while the IVD are constructed in such a manner that movements like bending and rotation of the spine are possible. The IVD is a poro-viscoelastic structure that can maintain very large loads without disintegrating. ${ }^{230}$ E.g., Nachemson ${ }^{151}$ found in vivo pressure in the nucleus of a lower lumbar IVD ${ }^{\text {a }}$ of a person weighing $70 \mathrm{~kg}$ to be as high as $2100 \mathrm{~N}$, depending on the position of the body and the weight that is carried. The IVD also functions as a shock absorber. ${ }^{48,177}$

Because of the presence of the hydrophilic PG, that attract water by means of osmosis, the disc is a highly hydrated vessel. It is sealed off by the annular rings and the carti-

elastic force viscous force osmotic force

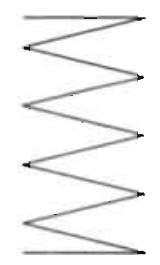

- clasticity of solid material

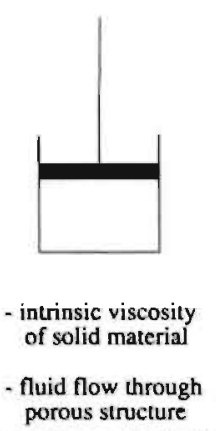

Figure 1.4: Schematic drawing of the forces that play a role in the mechanics of the intervertebral disc. laginous end plates. An increase in the axial (i.e., in the direction of the curve that follows the spine along its length) compressive force on the IVD causes the nucleus pressure to rise. As a result the annular fibres are circumferentially stretched as they are pushed outwards, i.e., the annular fibres take up stress in the circumferential direction, as the disc is axially compressed. During compression of the disc, tissue water is expressed out of the disc under the influence of the compressive force. Viscous forces (fluid-fluid and fluid-solid interaction) control the rate of fluid loss.

The forces that play a role in the mechanics of the intervertebral disc can be divided into a viscoelastic force associated with the stress-strain behaviour of the solid, an osmotic force associated with the presence of the hydrophilic PG, and a viscous force, associated with the relative motion of fluid and solid in the porous structure. These forces are depicted schematically in Figure 1.4.

\subsubsection{Poro-viscoelasticity and creep of the motion segment}

Mechanical testing of the motion segment (Figure 1.1) has concentrated on the response of the motion segment on various types of loading: axial compression, bending, rotation, and shear. In these studies poro-viscoelastic properties of the motion segment as a whole were determined, ${ }^{27,99,102,159,168,169,208}$ for canine motion segments, ${ }^{239}$ and in vivo porcine IVD. ${ }^{97,96}$ The poro-viscoelastic behaviour is caused by the intrinsic viscoelasticity of the

-This thesis focuses on the lower lumbar disc. 
annular fibres, and fluid flow inside the disc (indicated by the "poro" in poro-viscoelasticity). ${ }^{27,97,99,102,159,168,169}$

The relative importance of intrinsic viscoelasticity of the solid (energy dissipation due to relative solid-solid motion), and fluid flow (energy dissipation due to relalive fluid-solid and fluid-fluid motion) to creep of the IVD has been subject to controversial findings. Koeller et al. ${ }^{102}$ have demonstrated, using normal and desiccaled (very low water content) discs, that the creep and recovery characteristics of the disc are due to intrinsic viscoelasticity of annular fibres during extension and contraction, and not to fluid flow. Kraemer et al. ${ }^{103}$ maintain that creep is due to fluid flow. Kazarian ${ }^{84}$ found that a significant fraction of the disc's height loss under compression is likely to arise from fluid loss. Adams and Hutton ${ }^{4}$ found that a disc can loose as much as $20 \%$ of its fluid content, after having been loaded for 4 hours in vitro. Broberg ${ }^{27}$ in a study on height change of IVD in humans concluded that viscoelastic deformation of annulus fibres contributes approximately one quarter of the height change obtained after several hours normal activity, but dominates during the first hour. Poro-viscoelasticity is by some authors referred to as viscoelasticity, despite the fact that relative fluid flow evidently plays an important role in the time-dependent deformational behaviour of an IVD.

McNally and Adams ${ }^{138}$ measured horizontal and vertical stress profiles across cadaveric human discs under compression with a strain gauge transducer. The pressure sensitive membrane of the transducer was mounted on the side of a needle, and directed either with its surface parallel to the horizontal plane, or to the vertical plane. Ligaments and the vertebral arch had been left intact in these measurements. The stress they measured was partly a result from the hydrostatic fluid pressure and partly a result from the solid pushing against the sensing membrane. Their measurement results revealed little or no differences between the vertical and horizontal stress profiles in a central region of undegenerated or slightly degenerated discs. This central region, associated with elevated measured stress, was termed the functional nucleus by the authors, the remaining outer region with decreases stress was called the functional annulus. They concluded that the nucleus in those discs behaved like a fluid. However, horizontal and vertical stresses in the annulus did show differences, indicating that the direction of the annular fibres played a role in the measured stress. Measurements before creep in a disc under a purely compressive force (zero bending moment) yielded for young discs flat profiles for the functional nucleus, and decreasing stress in the functional annulus, with sometimcs local increases of stress shown in both horizontal and vertical profiles of the functional annulus. Older discs tended to have more irregular profiles with a much larger functional annulus. After creep (a $3 \mathrm{~h}$ period of compressive loading at $1200 \mathrm{~N}$ ) the overall sagittal diameter increased by $5-13 \%$, and disc height decreased by about $10 \%$, which is the same order of magnitude found in the diurnal variation observed in vivo. ${ }^{2,4}$ Creep frequently caused a considerable increase in vertical stresses in the posterior annulus when the discs were loaded with a pressure distribution, resulting in a constant flexion angle. ${ }^{138}$ The hydrostatic pressure in the nucleus after creep often fell to very low levels, indicating a high degree of stress shielding (absorbance of the compressive force) by the posterior annulus and the facet: joints. In an example McNally and Adams ${ }^{138}$ give, the size of the functional nucleus (the region of elevated compression prossure) had considerably decreased indicating loss of fuid from the nucleus, and a possible inward motion of annulus tissue.

The measurements of $\mathrm{McNally}$ and Adams ${ }^{138}$ on creep demonstrate that while the 
nucleus before creep has the highest stress and thus carries the major part of the compressive load, after creep this was done by the annulus. It should be noted however, that they surrounded their motion segments with a thin polyethylene film to minimize dehydration, not enabling the disc to absorb extra external water when under compression. In vivo, the discs are surrounded by interstitial fluid that can be attracted into the disc. ${ }^{27}$

Adams et al. ${ }^{3}$ found creep deformation in cadaveric lumbar spines subjected to static loading. The height changes found in the experiments were similar to the physiologic values of diurnal variation in lumbar disc height (about $1.5 \mathrm{~mm}$ ). ${ }^{2}$ Adams et al. ${ }^{3}$ found the water content to fall, the radial disc bulge (outward motion of outer annulus edge) to increase, the disc to become stiffer in compression and more flexible in bending, and the disc tissue to show mechanical changes. It became more elastic, and its affinity for water increased. Disc prolapse was provoked less easily with reduced water content. They concluded that different spinal structures are more heavily loaded at different times of the day, i.e., the stress distribution over the IVD and facet joints changes during the day.

It can be concluded, that the mechanics of the non- and slightly degenerate disc depends on the water content of the disc, and that this water content depends on the loading history of the disc. Under mechanical compressive loading, the disc creeps due to fluid flow and intrinsic solid viscoelasticity. The rate of deformation is thus governed by the poro-viscoelastic properties of the material. 


\subsubsection{Swelling of cartilaginous tissues}

Loss of disc water due to mechanical loading is compensated through attraction of water by a chemical pressure, ${ }^{134,219,222,224,225}$ which is generally called the osmotic, or Donnan osmotic pressure. Cartilage also attracts water, ${ }^{52,125,126,136,150,223}$ but swells much less (swelling is more restrained by the solid matrix) than IVD tissue. ${ }^{134,219}$ Whether a sample of cartilaginous tissue swells when in contact with an aqueous solution, depends on the relative magnitudes of the mechanical and chemical pressures, and the solid tensile stresses (mainly from collagen fibres) that restrain the swelling. The tissue swelling pressure is defined by Urban and Maroudas and colleagues as the external pressure that needs to be applied on the tissue to keep the tissue volume constant (at equilibrium). It can alternatively be defined as the pressure at which there is no driving force for fluid flow. ${ }^{130,222-224}$ In a formula:

$$
p_{\text {swell }}=\pi-\sigma_{e}=p_{\text {extern }} .
$$

In this equation $p_{\text {swell }}$ is the swelling pressure, which is the difference between the water attraction pressure $\pi$ and the effective stress, $\sigma_{\ell}$. The effective stress is defined as the total stress in the material minus the fluid pressure. It is also called the solid stress by some authors. pextern is the externally applied pressure.

The origin of the chemical attraction pressure for water in cartilaginous tissues is mainly found in the presence of the fixed negative charges of the macromolecular PG in the tissue. The fixed negative charges on the PG cause positive ions to be attracted to ensure bulk electroneutrality. This causes the total ion concentration inside the tissue to be larger than that outside the tissue. The imbalance of ions between the tissue and the external solution gives rise to an osmotic effect, called Donnan osmosis. The osmotic theory developed by Donnan ${ }^{50}$ gives a reasonable quantitative estimate of the water attraction pressure, exerted by cartilaginous tissue on its environment. The basic experiment, described by Donnan theory, is that of two solutions separated by a semipermeable nembrane. One solution contains multiply charged macromolecules (such as PG), cations and anions, and solvent (usually water), the other solution only contains cations, anions, and solvent (Figure 1.5).

The discussion of the osmotic phenomena in this thesis will be limited to monovalent ions. Ions with a higher valency pose problems that will not be addressed here. E.g., counterion condensation, the chemical binding of an ion to a fixed charge, occurs frequently with bivalent ions, and much less with monovalent ions. This phenomenon is not included in the present model. The molar concentrations: per unit fluid are indicated with the symbol c. Superscripts "+, -, f, s, pg" indjcate cations, anions, fluid, solid, and proteoglycans respectively. A single symbol $c$ 


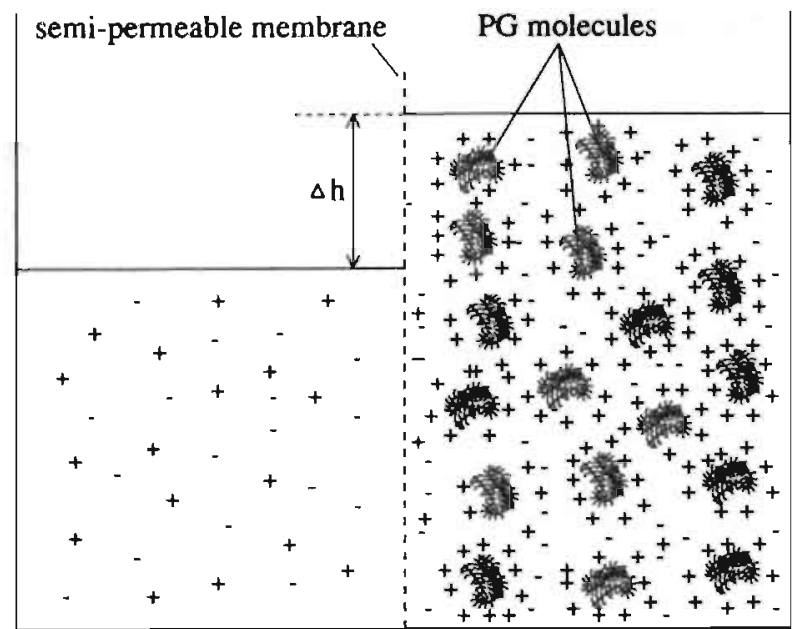

I: cations and anions

$$
\mathrm{c}_{\mathrm{l}}^{+}=\mathrm{c}_{\mathrm{l}}^{-}:=\mathrm{c} \quad \mathrm{c}_{\mathrm{ll}}^{+}=\mathrm{c}_{\mathrm{ll}}^{-}+\mathrm{c}_{\mathrm{n}}^{\mathrm{pg}} \text { or } \mathrm{c}^{+}=\mathrm{c}^{-}+\mathrm{c}^{\mathrm{pg}}
$$

Figure 1.5: Schematic representation of Donnan osmosis for monovalent ions: two compartments, separated by a semipermeable membrane, contain solutions of cations and anions (compartment I), and cations and anions and multiply charged macromolecules (PG; compartment II). The semi-permeable membrane is permeable to the solvent (water) and the monovalent ions, but not to the PG molecules. Due to the demand of bulk electroneutrality for each compartment, the concentrations of positive and negative charges must equal each other in each compartment. The concentration of the fixed charges on the macromolecules (FCD) is given by $c^{p g}$ in equivalent moles of univalent ions. The osmotic pressure resulting from the Donnan equilibrium is higher in the compartment with the PG molecules, indicated by the difference in height of the water columns $\Delta h$.

motic pressure for cartilaginous tissues. with no superscript is reserved for the concentration of the external salt. The fixed charge density (F(:D) is denoted by the symbol $c^{p g}$, which indicates the number of equivalent moles of a univalent ion. The situation of a sample of cartilaginous tissue, immersed in a salt solution, is analogous to the situation of two solutions, separated by a semi-permeable membrane. The sample of cartilaginous tissue is represented by the solution of charged macromolecules and small ions. The semi-permeable membrane is represented in the tissue itself, because the charged macromolecules are entangled in the tissue (Figure 1.3). and can not migrate out of the tissue into the external solution. The tissue is, however, permeable to the solvent (water), and the small ions. The analogy between two solutions, separated by a semipermeable membrane, and a sample of cartilaginous tissue, immersed in a salt solution, thus serves as a basis for the calculation of the Donnan os-

\section{Chemical potentials of ions and fluid in cartilaginous tissues}

Before turning to the theory of Donnan, it is imperative to explain the concept of chemical potential, because Donnan theory is based on that concept. The molar chemical potential of a component $i$ in a system, e.g. a mixture of gases, or a sample of cartilaginous tissue, immersed in a salt solution, is defined as the partial molar Gibbs free energy of the system at constant pressure, temperature, and numbers of moles of other constituents: ${ }^{38,92,179}$

$$
\begin{aligned}
& G=U-T S+p V, \\
& \mu^{i}:=\left(\frac{\partial G}{\partial n^{i}}\right)_{p, T, n^{\prime}},
\end{aligned}
$$


with:

$G=$ the Gibbs free energy of the system,

$U=$ the internal energy of the system,

$T=$ absolute temperature,

$S=$ the entropy of the system ${ }^{b}$,

$p=$ the fluid pressure,

$V=$ the volume of the system,

$\mu^{i}=$ the molar chemical potential of component $i$,

$n^{i, j}=$ the number of moles of component $i, j ; j$ refers to all components other than $i$.

Alternatively, the chemical potential is defined from the Helmholz free energy $F$ of the system, taking the partial differential with $n^{i}$ at constant volume, temperature, and numbers of moles of other components:

$$
\begin{gathered}
F=U-T S=G-p V, \\
\mu^{i}:=\left(\frac{\partial F}{\partial n^{i}}\right)_{V, T, n^{\prime}},
\end{gathered}
$$

with:

$F=$ the Helmholz free energy of the system.

Consider a system composed of a number of phases each open to component $i$. Such a system may be a sample of cartilaginous tissue together with its bathing fluid. The components capable of movement in such a system are the fluid and the small ions. The phases are defined as the external salt solution (I), and the tissue sample (II). In the situation where the pressure and the absolute temperature of the system are constant, and the system is at equilibrium, the Gibbs free energy is at a minimum. This is a consequence of the second law of thermodynamics (entropy law). Suppose that $\mathrm{d} n^{\mathrm{i}}$ moles from component $i$ are transferred from phase I to phase II. The loss of Gibbs free energy of phase I is $\mu_{I}^{i} \mathrm{~d} n^{i}$, and the gain of Gibbs free energy of phase II is $\mu_{I I}^{i} \mathrm{~d} n^{i}$. The total net gain of phases I and II together (the system) is $\mu_{I I}^{i} \mathrm{~d} n^{i}-\mu_{I}^{i} \mathrm{~d} n^{i}$. If the system is at equilibrium, i.e., the Gibbs free energy is at a minimum, the net increase of $G$ is zero, and hence: $\mu_{I}^{i}=\mu_{I I}^{i}$. This result can be generalized to any number of phases: at equilibrium the chemical potential of any component that can pass between them must be the same in each.

On account of the Maxwell relation $\left(\frac{\partial G}{\partial p}\right)_{T, n^{2}, n}=V$, the pressure dependence of the chemical potential is:

$$
\left(\frac{\partial \mu^{i}}{\partial p}\right)_{T, n^{i}, n^{\prime}}=\left[\frac{\partial}{\partial p}\left(\frac{\partial G}{\partial n^{i}}\right)_{p, T, n^{j}}\right]_{T, n^{i}, n^{j}}=\left[\frac{\partial}{\partial n^{i}}\left(\frac{\partial G}{\partial p}\right)_{T, n^{i}, n^{s}}\right]_{p, T, n^{\prime}}=\left(\frac{\partial V}{\partial n^{i}}\right)_{p, T, n^{j}}=\bar{V}^{i}
$$

\footnotetext{
${ }^{b}$ In the rest of this thesis, the symbol $S$ is reserved for the effective second Piola-Kirchhoff stress.
} 
where $V=$ volume, and $\bar{V}^{i}=$ partial molar volume of component $i$. The partial molar volumes are assumed constant, i.e., independent of composition and pressure. When we integrate (1.6) with respect to the pressure, assuming $\widehat{V}^{i}$ constant, we get:

$$
\mu^{i}=\mu_{c}^{i}+p \bar{V}^{i} .
$$

The constant of integration, $\mu_{c}^{i}$, may depend on temperature and composition (the variables that were kept constant during the integration), but is independent of pressure. From osmotic pressures measurements of dilute solutions, it was found that the pressure independent part of the chemical potential can be written as: $:^{74,92}$

$$
\mu_{c}^{i}=\mu_{0}^{i}(T)+R T \ln x^{i}
$$

where $\mu_{0}(T)$ is an integration constant, which may be a function of the temperature only, and $x^{i}=$ the molar fraction of the component $i$. For non-ideal (non-dilute) solutions an activity coefficient, $\gamma^{i}$, is added, that has a value between 0 and 1 :

$$
\mu_{c}^{i}=\mu_{0}^{i}(T)+R T \ln \gamma^{i} x^{i} .
$$

Inserting (1.9) in (1.7), the expression for the chemical potential becomes:

$$
\mu^{i}=\mu_{0}^{i}(T)+R T \ln \gamma^{i} x^{i}+p \tilde{V}^{i} .
$$

If we write the chemical potential in terms of the Helmholz free energy, using (1.3) and (1.4), we get:

$\mu^{i}=\left(\frac{\partial G}{\partial n^{i}}\right)_{p, T, n^{j}}=\left(\frac{\partial(F+p V)}{\partial n^{i}}\right)_{p, T, n^{j}}=\left(\frac{\partial F}{\partial n^{i}}\right)_{T, n^{\jmath}}+p\left(\frac{\partial V}{\partial n^{i}}\right)_{p, T, n^{j}}=\left(\frac{\partial F}{\partial n^{i}}\right)_{T, n^{j}}+p \bar{V}^{i}$.

Comparing (1.7) and (1.11), and using (1.9) we find:

$$
\left(\frac{\partial F}{\partial n^{i}}\right)_{T, n, s}=\mu_{0}(T)+R T \ln \gamma^{i} x^{i}
$$

where we see that the term $\left(\frac{\partial F}{\partial n^{i}}\right)_{T, n s}$ is independent of the pressure.

The molar chemical potential of a uni-univalent salt, $\mu^{ \pm}$, neglecting the pressure term, is defined as the sum of the separate chemical potentials of the cations and anions:

$$
\mu^{ \pm}:=\mu_{0}^{ \pm}+R T \ln \left(\left(\gamma^{ \pm}\right)^{2} x^{+} x^{-}\right),
$$

with:

$\mu_{0}^{ \pm}=$the standard molar chemical potential of the salt, ${ }^{\mathrm{c}}$

$\gamma^{ \pm}=\sqrt{\gamma^{+} \overline{\gamma^{-}}}=$average activity coefficient of the ions,

$x^{+}=$molar fraction of the positive ions,

$x^{-}=$molar fraction of the negative ions.

\footnotetext{
"We have left out the explicit dependence of $T$.
} 
We can neglect the pressure term $p \tilde{V}^{ \pm}$, with $\bar{V}^{ \pm}=$partial molar volume of the cations plus anions, because for the range of ion concentrations $(0.15-0.46 \mathrm{M})$, and fluid pressures: (0.08-0.2 MPa) considered in this thesis, it is small compared to the logarithmic term. ${ }^{d}$

For the fluid we define a chemical potential, $\mu^{f}$, using (1.10):

$$
\mu^{\prime}=\mu_{0}^{f}+R T \ln \left(\gamma^{f} x^{f}\right)+p \bar{V}^{f}
$$

with:

$\mu_{0}^{\prime}=$ the standard molar chemical potential of the fluid,

$\gamma^{J}=$ activity coefficient of the fluid,

$x^{f}=$ molar fraction of the positive fluid,

$\bar{V}^{f}=$ partial molar volume of the fluid.

\section{Donnan osmosis in cartilaginous tissues}

Regarding cartilaginous tissue as a mixture of a solid, a fluid, and positive and negative monovalent ions, we can write the mole fraction $x^{\alpha}$, of a component $\alpha$ in the mixture as follows:

$$
x^{\alpha}=\frac{c^{\alpha}}{\sum_{\alpha} c^{\alpha}}=\frac{c^{\alpha}}{c^{j}+c^{+}+c^{-}+c^{s}}, \quad \alpha=f,+,-, s .
$$

The molar concentration of the solid, $c^{s}$, is neglected because the molecular weight of the solid molecules ( $\mathrm{PG}+$ collagen) is high compared to that of the water molecules. The molar concentration of the water is approximated to the value of pure water (55 M). For the ionic concentrations considered in this thesis $(0.15-0.46 \mathrm{M})$, this is considered a reasonable approximation. For the calculation of the ionic molar fractions, $x^{+}$and $x^{-}$, their molar concentrations are neglected compared to the fluid molar concentration. We thus get:

$$
\begin{array}{r}
x^{f} \approx \frac{c^{f}}{c^{f}+c^{+}+c^{-}}, \\
x^{+} \approx \frac{c^{+}}{c^{f}} ; \quad x^{-} \approx \frac{c^{-}}{c^{f}} .
\end{array}
$$

In order for a sample of cartilaginous tissue to be in equilibrium with its environment (external salt solution), the chemical potentials of the fluid and the ions inside the tissue have to equal those of the external solution. In addition, the sample and external bath are required to be electrically neutral. ${ }^{38,92,179}$ The requirement for bulk electroneutrality of the external solution (Figure 1.5; compartment I) means that the concentrations of cations and anions equal each other. Inside the tissue (compartment II) the total negative charge ( $\mathrm{PG}$ molecules + anions) has to equal the total positive charge (cations):

$$
c_{I}^{+}=c_{I}^{-}:=c ; \quad c_{I I}^{+}:=c^{+}=c_{I I}^{-}+c_{I I}^{p g}:=\bar{c}^{-}+c^{p g} .
$$

\footnotetext{
${ }^{\mathrm{d}}$ E.g., for $\gamma^{ \pm}=0.7, c^{+}=c^{-}=0.46 \mathrm{M}, x^{+}=x^{-} \approx 0.008\left(x^{+} \approx \frac{c^{+}}{c^{\prime}}, x^{-} \approx \frac{c^{-}}{c^{\prime}}, c^{\prime}\right.$ = concentration of water $\approx 55 \mathrm{M}$ ), $p=0.2 \mathrm{MPa}, \bar{V}^{ \pm} \approx 0.0022 \mathrm{~m}^{3} \cdot \mathrm{mol}^{-1}$ (concentration of salt is $0.46 \mathrm{M}$ ), $R T=2.4^{*} 10^{5}$ $\mathrm{J} \cdot \mathrm{mol}^{-1}$, we get: $R T \ln \left(\left(\gamma^{ \pm}\right)^{2} x^{+} x^{-}\right)=-2.5^{*} 10^{10 \mathrm{~J}} \cdot \mathrm{mol}^{-1}$, and $p V^{ \pm}=4.4^{*} 10^{2} \mathrm{~J} \cdot \mathrm{mol}^{-1}$, the absolute of which is much smaller than the absolute of the logarithmic term.
} 
Because the concentrations of the cations and anions in compartment I equal each other, both concentrations are referred to with the symbol $c$, and the concentrations of ions and fixed charges in compartment II are referred to without the subscript "II".

The demand of equal chemical potentials for ions inside and outside the tissue during equilibrium, results in the following equation:

$$
c^{2}=c^{+} c^{-} .
$$

(1.19) was deducted using (1.13), (1.17) and (1.18), and assuming $\gamma_{I}^{ \pm}=\gamma_{I I}^{ \pm}$(which is valid for dilute solutions). For the fluid we again assume a dilute solution. The demand of equal chemical potentials (in equilibrium) for the fluid inside and outside the tissue leads to:

$$
p_{I}-\pi_{I}=p_{I I}-\pi_{I I},
$$

where $\pi_{I}$, and $\pi_{I I}$ are the ideal Donnan osmotic pressures for the external salt solution, and the tissue respectively:

$$
\pi_{I}=2 R T c ; \quad \pi_{I I}=R T\left(c^{+}+c^{-}\right)=R T\left(2 c^{-}+c^{p g}\right) .
$$

$(1.20)$ and (1.21) were derived using (1.14), (1.16), and (1.18), and the approximations $\ln (1 /(1+x))=-x, c_{I}^{f} \bar{V}_{I}^{f}=c_{I I}^{\prime} \bar{V}_{I I}^{\prime}=1$.

When we substitute (1.21) into (1.20) we get:

$$
p_{I I}-p_{I}=\pi_{I I}-\pi_{I}=\Delta \pi=R T\left(2 c^{-}+c^{p g}-2 c\right) .
$$

Equation (1.22) shows that the a hydrostatic pressure difference between compartment $I$ and II exists to compensate for the Donnan osmotic pressure difference $\Delta \pi$ (also frequently called the Donnan osmotic pressure; Figure 1.5).

For cartilaginous tissues departure from ideality (e.g., for non-dilute solutions) is accounted for by introducing osmotic coefficients, which generally are different inside and outside the tissue. The non-ideal Donnan osmotic pressure difference thus is:

$$
\Delta \pi=R T\left(\Phi_{I I}\left(2 c^{-}+c^{p g}\right)-\Phi_{I}(2 c)\right),
$$

where $\Phi_{I I}$ is the osmotic coefficient inside the tissue, and $\Phi_{I}$ is the osmotic coefficient of the external bath. 


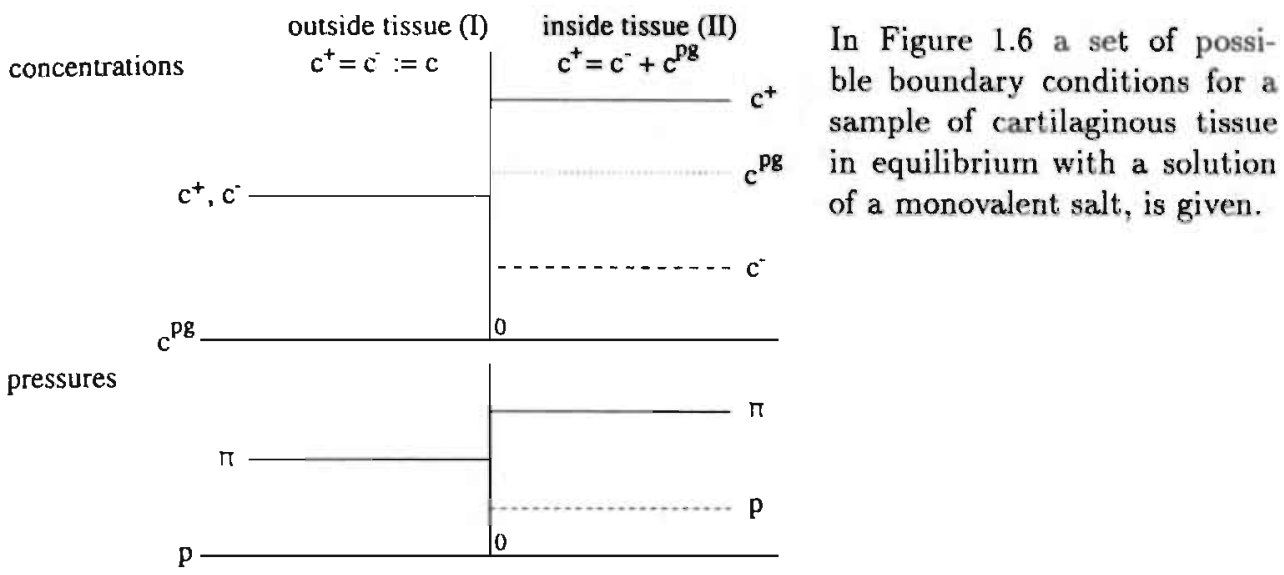

chemical potentials

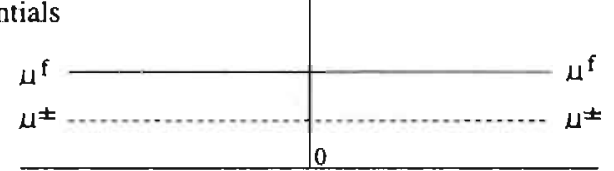

Figure 1.6: Schematic overview of a possible set of boundary values for a sample of cartilaginous tissue, in equilibrium with a solution of a monovalent salt. Generally, the concentrations of the cations $\left(c^{+}\right)$, and anions $\left(c^{-}\right)$, as well as the fluid $(p)$, and osmotic $(\pi)$ pressures are different outside and inside the tissue, in contrast to the chemical potentials of the ions $\left(\mu^{ \pm}\right)$, and the fluid $\left(\mu^{f}\right)$. Note that the fluid pressure outside the tissue has been arbitrarily set to zero.

\section{Electrical potentials in cartilaginous tissues}

The Donnan equilibrium for cartilaginous tissues predicts concentration gradients of cations and anions across its boundaries (the semipermeable membrane; Figures 1.5 and 1.6). From compartment I to compartment II the cations concentration increases and the anion concentration decreases. These concentration gradients cause - and are subsequently counteracted by - an electrical potential difference across the boundary: The Nernst or Donnan potential. ${ }^{33,68,166}$ The Donnan potential for a given ionic species is calculated from the equilibrium condition for the electrochemical potential of the ion. The electrochemical potential for an ionic species $i$ equals the chemical potential, plus an electrical term that accounts for electrical potential gradients: $:^{92,179}$

$$
\mu_{E}^{i}:=\mu_{0}^{i}+R T \ln \left(\gamma^{i} x^{i}\right)+z^{i} F_{a} \Psi_{E}
$$

with:

$\mu_{E}^{i}=$ the electrochemical potential for the ionic species $i$,

$z^{i}=$ the valency of the ion,

$F_{\alpha}==96,487 \mathrm{coul} / \mathrm{mol}$, the Faraday constant,

$\Psi_{E}=$ the electrical potential relative to a potential in a reference state. 
If the electrical potential in the salt bath is taken as the reference potential, the potential in the tissue in the equilibrium state is the Donnan potential, $\Psi_{D}$, which can be written in terms of $c^{+}$, or $c^{-166}$ We combine expression (1.24) to equal the electrochemical potentials for the cation in the salt bath (compartment I) and the tissue (compartment II) with eqs. (1.17) and (1.18), and get:

$$
\Psi_{D}=\frac{R T}{F_{a}} \ln \frac{\gamma_{I}^{+} c}{\gamma_{I I}^{+} c^{+}},
$$

where $\Psi_{D}$ is the Donnan potential.

In this thesis we consider combinations of cations and anions, that travel in neutral pairs. We therefore defined the chemical potential of the salt molecules, which is the sum of the electrochemical potentials of the separate ions. Because the electrical potential term for the anions is the negative of that for the cations, these terms cancel, and expression (1.13) is obtained upon summation of the electrochemical potentials of the cations and anions. When ions are assumed to move in neutral pairs, the Donnan potential does not influence their movement, and the chemical potential of the salt can be used as the driving force for the salt molecules.

Movement of ions across a boundary of and inside a sample of cartilaginous tissue goes accompanied by another potential difference that is caused by the presence of the fixed charges in the tissue. The negative fixed charges attract cations, and repel anions. In a situation when fluid with ions flows relative to the solid (fixed charges), the cations are separated from the anions, resulting in a potential difference in the direction of the fluid flow: the streaming potential. ${ }^{74,92,125}$ During a diffusion process, a similar situation occurs, resulting in a diffusion potential. Streaming and diffusion potentials may influence ions diffusion, and fluid flow to and from cartilaginous tissues. ${ }^{125}$

In order to model the effects of electrical potentials in the mechanics of cartilaginous tissues, one should define the electrochemical potentials of the cations and anions separately in the model. It should be noted that the effects of the streaming and diffusion electrical potentials only play a role in transitional situations, where the tissue is not in equilibrium with its environment. In the model for cartilaginous tissues that is used in this thesis, the streaming and diffusion potentials are not included.

\section{Other osmotic and swelling forces}

Swelling of cartilaginous tissues can also be explained in terms of the electrostatic charge to charge repulsive forces that the PG exert on each other (Figure 1.3b). This way of describing tissue swelling can be seen as an alternative for Donnan osmosis. In cartilage, aggregates of the PG may be restrained to $1 / 10$ th of their volume in free solution. ${ }^{72,148}$ At this concentration their fixed negative charges are so close to each other that they repel each other, resulting in swelling of the tissue. This charge to charge repelling force is called the chemical stress, ${ }^{52.53}$ or the chemical expansion stress. ${ }^{109}$ The chemical ex pansion stress, like the Donnan osmotic pressure, depends on the concentration of fixed charges (FCD), and the ions concentration in the external solution. The external ions concentration determines the internal ions concentrations via the demands of bulk electroneutrality and identical chemical potentials for ions and fluid in equilibrium situations. 
The internal cations concentration determines the amount of charge shielding the fixed charges of the PG experience between themselves. The more cations that are present between the fixed charges of the $\mathrm{PG}$, the less these charges will repel each other. ${ }^{\circ}$

In the situation where the large molecules do not carry electrical charges, water would be attracted into the tissue as a result of an osmotic pressure difference due to the presence of the large molecules. This type of osmosis for cartilaginous tissues has been termed excluded volume osmosis. ${ }^{52,223}$ Its magnitude was found to depend on the number of large molecules, ${ }^{223}$ notwithstanding the fact that these molecules are entangled.

Summarizing we have the following components that can explain the chemical attraction of water by cartilaginous tissues:

1. the Donnan osmosis component, $52,53,109,223$

2. the chemical expansion component, ${ }^{52,53,109}$

3. the excluded volume osmosis component, ${ }^{52,223}$

The nature of the third of these components is non-electrical. Urban et al. ${ }^{223}$ examined osmotic pressure from extracted PG from cartilage and IVD, and concluded that the size and degree of aggregation had no measurable influence on the osmotic pressure, and that the excluded volume component under physiological conditions contributed for not more than $15 \%$ to the total osmotic pressure.

The two PG electrical charge associated forces, Donnan osmosis and chemical expansion, can both be used to model swelling phenomena with fixed electrical charges. In the case chemical expansion is used to model the swelling behaviour, an extra term is added to the stress in the stress-strain law of the tissue (chemical stress; Eisenberg and Grodzin$\mathrm{sky}^{52,53}$ ), or to the stress-strain law of the solid matrix (chemical contraction force; Myers et al. ${ }^{150}$ ). Lai et al. ${ }^{109}$ developed a chemo-mechanical theory that uses the Donnan theory to predict osmotic pressure and ion distribution, and at the same time model the chemical expansion stress as a term in the stress-strain relationship. They maintained that without chemical expansion stress, one-dimensional free swelling experiments can not be modelled correctly. However, Snijders ${ }^{200}$ et al. ${ }^{203}$ fitted one-dimensional swelling and compression experiments using Donnan osmosis alone without chemical expansion stress.

\section{Extra- and intrafibrillar water}

Urban and McMullin, ${ }^{224,225}$ Maroudas and Bannon, ${ }^{130}$ and Maroudas and collcagues ${ }^{93,135,252}$ have shown that the Donnan equilibrium model, although used by many authors, ${ }^{1019,203}$ is oversimplified. The cartilaginous sample cannot be viewed as having a single fluid compartment. They distinguish between extra- and intrafibrillar compartments. In rartilaginous tissues, a certain fraction of water is trapped insicle the collagen fibrils. This fraction of the total tissue water is called the intrafibrillar water. The fraction of intrafibrillar water was found to depend on the osmotic pressure difference between the intraand extrafibrillar compartments. ${ }^{93,135}$

\footnotetext{
eThe amount of charge shielding can be expressed in the electrical interaction distance (Debye length) buween the fixed charges. The greater the charge shielding, the greater the Debye length.
} 
The PG, because of their large size, are excluded from the intrafibrillar space. Therefore, the fixed charge density should be based on the amount of extrafibrillar water, because only then swelling pressures of cartilaginous tissues can be predicted from swelling pressures of proteoglycan solutions. This has been found both for cartilage, ${ }^{130,136}$ and IVD. ${ }^{224}$ The effective concentration of PG, the effective FCD, is thus higher than the value based on total tissue water. The osmotic pressure, estimated from extrafibrillar or effective FCD is correspondingly higher than that based on total tissue water. 


\subsubsection{Tissue deformation: poro-viscoelastic properties of the IVD and IVD annulus}

The equilibrium deformation of IVD tissue and other cartilaginous tissues is governed by the balance of mechanical and chemical loads, and the solid stress. Consider a sample of tissue equilibrated in an external salt solution experiencing a constant mechanical pressure. When the mechanical pressure is increased stepwise, the solid creeps, changing the solid stress, and fluid is squeezed out of the tissue. This increases the FCD ( ${ }^{p g}$ on fluid volume basis), and causes an increase in the Donnan osmotic pressure inside the tissue $\left(\pi_{I I}\right.$ in eq. (1.21)). The increased osmotic pressure inside the tissue results in a decrease of fluid expression out of the tissue. Eventually, a new equilibrium is reached where the mechanical and chemical loads are again balanced by the solid stress. In the new equilibrium situation the ions concentrations, FCD, and solid stress and strain are generally different.

The transition from one equilibrium state to the other is governed by the poroviscoelastic properties of the tissue. These include the diffusion properties for the small ions involved, the hydraulic permeability (permeability coefficient) for fluid flow, and the intrinsic viscoelasticity of the solid. The osmotic swelling properties (osmotic coefficients, F(DD, ionic activities) also determine the transitions from equilibrium states. Osmotic swelling has been treated in the last section. Here, we will focus on the poro-viscoelastic properties of the IVD.

\section{Exchange of fluid and solutes, diffusion of small ions}

In an intact IVD, fluid exchange and the exchange of small solutes, such as $\mathrm{Na}^{+}, \mathrm{K}^{+}$, $\mathrm{Cl}^{-}, \mathrm{SO}_{4}^{2-}, \mathrm{Ca}^{2+}$, with its environment goes along two pathways: via thr annulus and via the end plates. The relative importance of these two routes for the fluid depends on the spatial variation of the magnitude of the permeability coefficient, and the mechanical and osmotic pressure gradients. ${ }^{27,159}$ Ohshima and colleagues ${ }^{159}$ examined the uptake and washout of tritiated water in the pig tail IVD. The molar partition coefficient of ${ }^{3} \mathrm{H}_{2} \mathrm{O}$ was found to be almost equal to 1 even under a compression load, which suggests that water is freely exchangeable. The diffusion of ${ }^{3} \mathrm{H}_{2} \mathrm{O}$ in the unloaded disc for both uptake and washout was about 2 to 3 times larger in the peri-annular route than in the end plate route.

The diffusion of solutes plays a role in the swelling of cartilaginous tissues as the. distribution of these solutes determines the magnitude of the osmotic pressure gradicnt. For the transient behaviour (time-dependent transition from one equilibrium state to another) it is therefore important to know the rate at which a solute moves by molecular diffusion. This rate is characterized by the diffusion coefficient $D$, which is an increasing function of the temperature. Solutes diffuse more slowly in the disc than in free solution. This is due to the presence of solids in the tissue, which act as obstacles, and effectively increase the path length to cover a certain distance (increased tortuosity). A formula, derived by Mackie and Meares ${ }^{19}$ for porous media and based on purely geometrical considerations relates the diffusion coefficient in the tissue, $D^{\prime}$ to that in free solution 
$D_{\text {free: }}^{l}$

$$
\frac{D^{l}}{D_{f r e t}^{l}}=\frac{\left(\dot{o}^{\prime}\right)^{2}}{\left(2-\dot{\phi}^{f}\right)^{2}},
$$

where $\phi^{f}$ is the volume fraction of the fluid. It was concluded by Maroudas ${ }^{129}$ that even at the extreme ends of the physiological range it appears that the reduction of the diffusion coefficient can be estimated reasonably well from equation (1.26). For the small charged solutes $\mathrm{Na}^{+}$and $\mathrm{Cl}^{-}$that play a role in Donnan osmotic swelling, Urban ${ }^{216}$ measured the diffusion coefficients in the disc at $4{ }^{\circ} \mathrm{C}$, and from these values calculated values at $25^{\circ} \mathrm{C}$ (units are ${ }^{*} 10^{-9 *} \mathrm{~m}^{2} / \mathrm{s}$ ): $\mathrm{Na}^{+}: 0.518$ at $4^{\circ} \mathrm{C}, 0.86$ at $25^{\circ} \mathrm{C} ; \mathrm{Cl}^{-}: 0.806$ at $4^{\circ} \mathrm{C}, 1.35$ at $25^{\circ} \mathrm{C}$.

For small solutes, Urban et al. ${ }^{217}$ found that the route for uptake in the canine IVD (in vivo study) depends on the charge of the solute. For a small anion such as sulphate, the amount supplied through the periphery of the annulus is almost twice as much as that diffusing through the end plates. For a small uncharged solute such as glucose the two routes are of equal importance, while for cations the end plate route is more effective. In another study, also on dogs, Urban et al. ${ }^{215}$ concluded that small solutes, e.g. $\mathrm{O}_{2}$, glucose, and sulphate are transported into the disc chiefly by diffusion. For large solutes bulk fluid flow might be of more importance, because of their low diffusivities.

\section{The permeability coefficient}

The permeability coefficient for fluid flow determines at a given driving force the rate of How, and is thus important for the mechanical functioning and possibly important for the transport of large solutes of the IVD. ${ }^{215}$ For cartilage, research has been done on the permeability coefficient as a function of distance from the articular surface (measurements in permeability tube). ${ }^{125,128,149}$ The permeability coefficient was found to increase toward the articular surface. In some cases there was an increase of the permeability coefficient from the deeper zones to the superficial layers and then a decrease to the articular surface. Measurements of the permeability as a function of fixed charge density (FCD) and collagen content yielded an inverse relationship for both, i.e., permeability decreased as FCD, and/or collagen content increased. This was found for FCD by Maroudas, ${ }^{125}$ and for FCD and collagen by Muir et al. ${ }^{149}$

Mansour and Mow ${ }^{122}$ measured the permeability in bovine cartilage in a permeability tube as a function of compressive strain and high pressures. They found that the permeability coefficient decreased linearly with the strain, and non-linearly with the pressure.

To date, little has been published about the permeability coefficient of the IVD. Urban and Maroudas ${ }^{22}$ determined the permeability coefficient of human radial annulus samples, and axial nucleus samples, and gave its variation with hydration for one disc. They found that the permeability rose with hydration. Best et al. ${ }^{17}$ determined the biphasic permeability coefficient $\left(K_{b i}\right)$ from confined compression experiments of human L3-4, and L4-5 discs. They found no differences in $K_{b i}$ for different regions and radial positions in the disc. Drost et al. ${ }^{51}$ tested canine IVD annulus, also in confined compression experiments (L4-5, L5-6, L6-7, L7-S1), and found no significant differences in the permeability coefficient for different regions in the disc. They did not test the radial dependence of $K_{b i}$. 


\section{Viscoelastic properties}

Tensile tests have been performed to determine the viscoelastic properties of the annulus fibrosus. The tensile properties of the annulus vary with sample orientation, ${ }^{61,123}$ location in the disc, ${ }^{61}$ and tissue hydration. ${ }^{61,167,169}$ Albeit small in magnitude, there was also a variation found with age (position was found to be a more important factor). ${ }^{1} \mathrm{Wu}$ and $Y{ }^{236}$ tested annulus samples and compared the results with those of a theory of fibrous materials. Skaggs et al. ${ }^{199}$ tested single lamella AF specimens. They found a non-linear stress-strain relationship, and significant radial and circumferential variations in tensile properties, the anterior being stiffer than the posterolateral regions, and the outer being stiffer than the inner regions. Also, they found the tensile modulus of adjacent single layer specimens with opposite fibre orientation not to vary with orientation.

Compressive mechanical properties of the annulus and the confined swelling and compression experiment

Few studies have been conducted to assess the compressive mechanical properties of IVD annulus. Best et al. ${ }^{17}$ measured the compressive mechanical properties of the human lumbar annulus fibrosus, and tested their relationship to biochemical composition, as well as their regional variation. Drost et al..$^{51}$ compared the permeability, compressive or aggregate modulus, and transport coefficient of axial versus radial specimens. Both studies were done with confined compression, ${ }^{17}$ and swelling and compression ${ }^{51}$ (CSC) experiments. Biphasic theory ${ }^{145,211}$ was used to determine the mechanical parameters.

The CSC experiment is used for the determiuation of mechanical properties, and at the same time it can be used for the assessment of mechanical models of the IVD. In the experiment a cylindrical sample of annulus material is confined in a cylindrical chamber. Compressive load is conferred to the sample by means of a loading piston that fits tightly in the chamber. The sample is placed in contact with a bathing solution of known osmotic strength by placing it on a filter through which the solution circulates (Figure 1.7a). The sample's height variation (piston displacement) in time as a function of mechanical and chemical load is measured. In Figure $1.7 \mathrm{~b}$ an example of a time-sample hight curve of a CSC experiment is given. 
a.

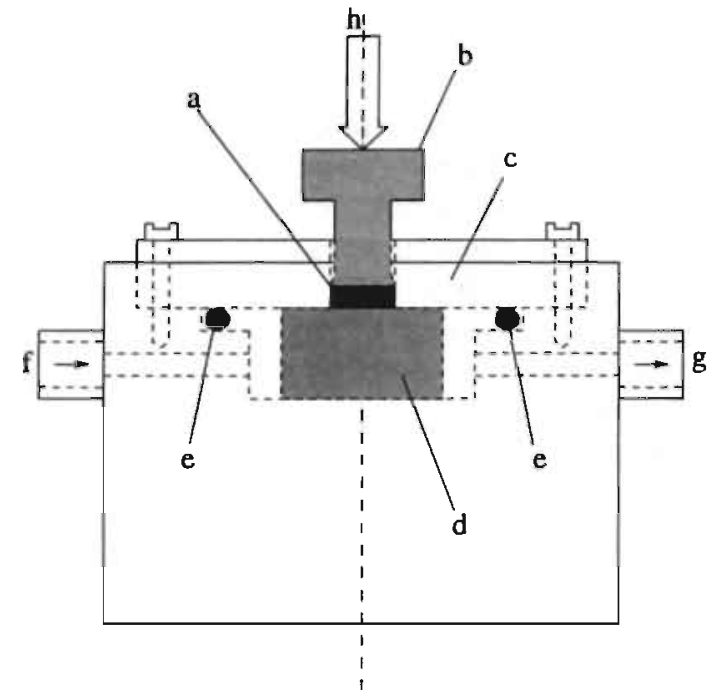

b.

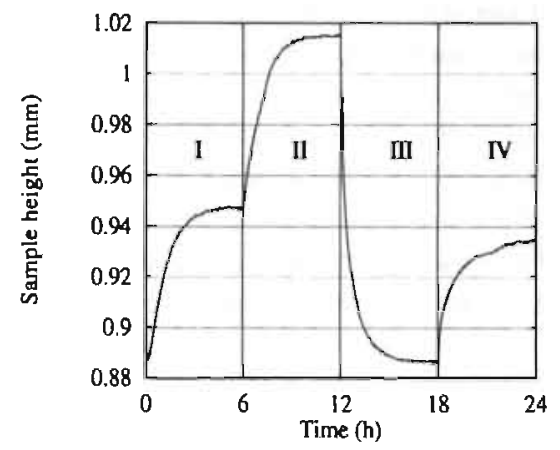

Figure 1.7: Schematic view of the measurement chamber of a confined swelling and compression experiment (a), and an example of a time-(sample beight) curve of such an experiment (b). One can discern 4 phases (I: conditioning, Il: swelling, III: compression, IV: control). Between each two phases the mechanical pressure on the sample, and/or the osmotic strength of the bathing solution is changed, resulting in a transient from one equilibrium situation (at the end of each phase) to another. Explanation of the symbols in (a): a: sample, b: piston, c: cover platen, $d$ : filter, e: O-ring, $f$ : fluid inlet, $g$ : fluid outlet, h: mechanical load.

In the CSC experiment the time-dependent deformation of the tissue is expected to be largely governed by the poro-elastic properties (relative fluid flow through an elastic solid) of the tissue, rather than the intrinsic viscoelastic property of the solid, because fluid can be exchanged freely with the external salt bath. Using a poro-elastic model to describe the mechanical behaviour of the tissue in this type of experiment therefore seems appropriate. 


\subsection{Models of IVD and other cartilaginous tissues}

Theories that describe the mechanical behavjour of cartilaginous tissues can be divided into three categories.

1. The physico-chemical theories that are based on microscopic or macroscopic continuum electrical models. The classical Donnan theory for aqueous polyelectrolyte solutions is an example of the macroscopic continuum electrical model. Physicochemical theories do not explicitly model the solid phase.

2. Solid (single phase) or solid-fluid (biphasic) continuum theories that do not explicitly model PG electrical charge related swelling phenomena.

3. Solid-fluid models that take ionic effects into account by either implicitly modeling them (hybrid biphasic models), or explicitly modeling the ionic phase (triphasic models).

\subsubsection{Physico-chemical models}

The mechanical behaviour of cartilaginous tissues, especially with regards to the transport of fluid (swelling) and solutes (nutrition), charged or uncharged, is largely influenced by their fixed charges. ${ }^{52,68,70,109,125-128,131,203,217,218}$ In the approach to the modeling of fixed electrical charges related phenomena in cartilaginous tissues there are two possible models: a macroscopic continuum model, or a microscopic continuum model. In the microscopic continuum approach the tissue is modeled as having a space varying electrical potential between the charged molecules in the matrix. ${ }^{11,33,68}$ The Poisson-Boltzmann (PB) cell. model is used to predict electrostatic interactions, osmotic pressures, ion distributions, and ion activity coefficients. ${ }^{33}$ An example of a PB unit cell for cartilaginous tissues consists of a cylindrical model of a GAG chain (the PG side chain). ${ }^{33}$ The characteristic radius of such a cell is in the order of the intercharge distance along the GAG chain (0.64 $\mathrm{nm}$ for cartilage ${ }^{42,174}$ ).

Macroscopic continuum models, in contrast to microscopic continuum models, assume smooth volume fixed charge density, potential profile, and mobile ion concentration profile. ${ }^{68}$ Characteristic dimensions in this continuum contain many tissue molecules, and are so large compared to a Debye length (the characteristic decay length in an electrostatic field) that quasi-electroneutrality is assumed to be valid everywhere in the tissue phase. In equilibrium, mobile ions are assumed to be distributed according to the Donnan theory. Quasi-neutrality is violated only at the tissue/electrolyte bath interfaces, where the potential changes steeply on the scale of a Debye length from its intratissue value to that of the electrolyte bath (Donnan potential; eq. $(1.25)^{33,166}$ ).

Buschmann and Grodzinsky ${ }^{33}$ compared the macroscopic Donnan and microscopic $\mathrm{PB}$-cell models, and concluded that for the range of $\mathrm{PG}$ concentrations in adult bovine articular cartilage there is a better correspondence between the PB-cell model predictions of swelling pressure and measurements, than for the ideal Donnan model. Bulk small ion partitioning was acceptably predicted by the ideal Donnan model. Basser and Grodzinsky ${ }^{11}$ also compared these models and found that the Donnan approximation is accurate at low ionic strength, but less faithful at physiologic and higher ionic strength. In these 
cases osmotic coefficients have to be introduced in the Donnan theory to account for the non-ideality.

Although the PB-cell models seem to be more accurate in predicting electromechanical behaviour of cartilaginous tissues, they are numerically complex and have not been widely used. Most researchers have employed the macroscopic (non-ideal Donnan) theory to interpret and predict electromechanical phenomena of soft charged tissues. Macroscopic physico-chemical models can be used to explain fluid and solute transport in soft hydrated tissues, $125-128,131,132,215,217,218,222$ and small ions distribution and swelling of these tissues. ${ }^{130,134,136,220,222-225}$ As these models do not explicitly take the solid phase into account, they are not able to account for deformational behaviour of the tissue. Neither can they be used for the modeling of failure mechanisms of the solid.

\subsubsection{Single phase and biphasic continuum models}

The development of mechanical models that explicitly model the solid phase of soft charged tissues has started with single-phase models: e.g. elastic, ${ }^{73}$ and single phase viscoelastic. ${ }^{60}$ Because of the importance of fluid flow in the tissue relative to the solid matrix, biphasic models were developed. These models define immiscible solid and fluid phases that are both intrinsically incompressible. The sum of the phases is called the mixture. A detailed theoretical treatment on a general mixture of $\mathrm{N}$ phases is given by Bowen. ${ }^{25}$ The interaction of the phases in mixture cumplies with the laws of thermodynamics: conservation laws (mass, momentum, moment of momentum, energy), and increase of entropy for the total mixture. In a biphasic mixture momentum transfer of one phase to the other is possible through frictional drag. This kind of interaction has been used to account for the apparent viscoelastic properties in the compression of hydrated soft tissues. ${ }^{144}$ Another way to do this is by defining a constitutive relationship for an intrinsic viscoelastic solid. ${ }^{120}$ Biphasic theories have been extended to include other non-linear behaviours such as strain-dependent permeability, ${ }^{81,110}$ and finite (non-linear) deformation. ${ }^{80,108,146}$

\subsubsection{The triphasic model}

It is the merit of these biphasic theories that they have been reasonably successful in describing compressive behaviour of cartilaginous tissues, treating the collagen-proteoglycan matrix as a solid with the time-dependent properties depending on all the underlying physico-chemical effects. However, biphasic theories are not equipped to model phenomena such as Donnan osmotic and chemical expansion swelling. These PG charge related phenomena are responsible for the stressed state of the collagen fibres, and increased fluid pressure, inside a tissue sample bathed in a solution of $\mathrm{NaCl}$, when there is no external mechanical load. This is often referred to as the collagen pre-stress or elastic stress in the solid matrix. ${ }^{69,127,128,130}$ Several researchers have attempted to incorporate the ionic effect, but have done so in varying and incomplete ways (hybrid biphasic models). ${ }^{52,53,112,113,147,150,193,194}$

The first theory to model the ionic effect explicitly was reported by Lai et al. ${ }^{109}$ Their theory incorporates the Donnan ion distribution and osmotic pressure theory for polyelectrolyte solutions. In addition, PG chemical expansion stress is incorporated to 
account for swelling also. They define three phases: (1) the interstitial fluid phase and (2) the collagen-proteoglycan solid phase, both immiscible, and a fluid miscible phase: (3) the ionic phase. In this triphasic theory, the stresses in the solid matrix and the chemical potentials for the interstitial fluid and ions are related to the Helmholz energy functions in accordance with the laws of energy balance and entropy. The triphasic theory thus unites the continuum biphasic theories, ${ }^{145,146}$ and macrocontinuum physico-chemical theories $^{50,127,128,219,220}$ for the mechanics of cartilaginous tissues.

Snijders ${ }^{200}$ developed a similar triphasic theory, based on the theory of Lai et al.. The main difference between the two theories is that Snijders neglects chemical expansion stress, but uses only Donnan theory to account for the swelling.

\subsubsection{Finite element models}

The complexity of the bi- and triphasic continuum mixture models as a result of geometrical complexity (IVD, motion segment, diarthrodial joints), and non-linear, time dependent behaviour, calls for numerical methods to solve them. Many researchers have chosen the finite element (FE) method as the numerical approach to solve the equations of their models, as this method is the most appropriate due to its ability to solve problems of complicated geometry, material properties, and loading. Many FE models have been constructed for the intervertebral disc, ${ }^{14,111,154,178,186-188,204,205}$ motion segment, ${ }^{65,105,116,155,186,190-192,196,197,214}$ and spine, ${ }^{189}$ in relation to its normal functioning, but also dysfunctioning.

Ion swelling was introduced in a FE model by Simon and Gaballa. ${ }^{195}$ They used an uncoupled differential equation for the ion diffusion, and imposed its effect upon the porcelastic problem. The degrees of freedom were the solid displacement $\boldsymbol{u}$, a relative fluid displacement parameter and the local ion concentration. Snijders et al. ${ }^{203}$ have developed an FE model based on their triphasic theory with coupled differential equations. 'This formulation used as degrees of freedom the solid displacement $\boldsymbol{u}$, the fluid pressure $p$, and the ions mass density $\rho^{ \pm}$. To solve the differential equations they used the weighted residual method and a total Lagrange formulation. Snijders et al. demonstrated the ability of the model (1) to fit confined swelling and compression measurements of annulus fibrosus tissue $^{202}$ and (2) to simulate compression of an axisymmetric intervertebral disc. ${ }^{201}$

Snijders et al. paid particular attention to the correct handling of pressure and concentration fields within the domain and at the boundary of the domain. The classical requirement for the integral

$$
\int \frac{d^{n} f}{d X^{n}} d V
$$

to be defined, is the continuity of

$$
\frac{d^{n-1} f}{d X^{n-1}}
$$

within the domain V. In section 1.3 .2 (Figure 1.6) it is shown that generally $p, c^{+}$and $c^{-}$ are not continuous when $c^{p g}$ is not continuous. This is the case, e.g., at the boundaries of a sample of cartilaginous tissue, but discontinuities can also play a role inside a sample. One can imagine that at places where fissures occur in the solid structure, the fixed charge 
density and fluid fraction are also discontinuous. The ions density is defined by:

$$
\rho^{ \pm}=\phi^{f}\left(M^{-} c^{-}+M^{+} c^{+}\right),^{\mathfrak{f}}
$$

where $M^{i}$ are molar masses, and $\phi^{f}$ is the fluid volume fraction. From (1.29) it can be seen that $\rho^{ \pm}$is not continuous when $c^{+}, c^{-}$, or $\phi^{\prime}$ are not continuous. We can thus conclude that generally $p$ and $\rho^{ \pm}$are not continuous when $c^{p g}$, or $\phi^{f}$ are not continuous, and that this is always the case at the boundaries.

As $p$ and $\rho^{ \pm}$are only piecemeal continuous, only piecemeal integration of the integrals $\int \nabla p d V$ and $\int \nabla \rho^{ \pm} d V$ is possible. Special interface elements were designed by Snijders et al. ${ }^{203}$ in order to cope with pressure and density (concentration) jumps along the element boundaries, using a penalty method.

Snijders has implemented the numerical formulation of Snijders et al. ${ }^{203}$ with boundary elements in the commercial finite element software package DIANA. Code was developed for $1-, 2-$, and $3-\mathrm{D}$ elements.

'Note that eq. (1.29) is not an expression for the $\rho^{ \pm}$of a neutral salt, as is the $\rho^{i}$ in Lai et al. ${ }^{109}$ Inside the swelling material generally $c^{+} \neq c^{-}$. 


\subsection{Aims and contents of this thesis}

The development and assessment of the tiriphasic micromodel of cartilaginous tissues, and the determination of mechano-chemical characteristics of the annulus fibrosus of the intervertebral disc is the topic of this thesis. The triphasic model views cartilaginous tissues as a superposition of three continua (components): the solid, fluid and ions components. The model incorporates Donnan osmosis to account for the swelling of the material.

Sophisticated models as the triphasic model can only be solved numerically. The finite element method (FEM) has been successfully employed by Snijders et al. ${ }^{203}$ However, Snijders et al. used degrees of freedom that are not continuous when certain material properties of the tissue (such as the fixed charge density and the fluid fraction) are not continuous, which is the case at boundaries of the material, but can also occur inside the material, when, e.g., discontinuities occur in the solid properties. In these cases it is more convenient to base the numerical formulation on degrees of freedom that are continuous, regardless of discontinuities in the material properties. A logical choice for continuous degrees of freedom is that of the chemical potentials of the ions and the fluid (next to the solid displacement, which is also used in the FEM of Snijders et al ${ }^{203}$ ). In this thesis a numerical formulation based on the chemical potentials of ions and fluid, and the solid displacement is developed (chapters 2 and 3 ) and successfully employed to simulate onedimensional confined swelling and compression experiments of samples of IVD annulus fibrosus.

The view of cartilaginous tissue as a superposition of solid, fluid and ions has as a consequence that the properties of the components in such a model are separatind. This yields a physically realistic picture of the material: the influence of changes in the properties of the separate components can be studied. One advantage of this lies in the domain of the development of model materials, that mimic the properties of the real tissue. It is the merit of the triphasic model that it is capable of separating the elastic (solid), viscous (fluid), and osmotic (ions) contributions to the mechano-chemic'al behaviour of cartilaginous tissues. This separation turned out to be insufficient to explain material swelling quantitatively. As was shown by Urban and McMullin, ${ }^{24,}{ }^{225}$ Maroudas and Bannon, ${ }^{130}$ and Maroudas and colleagues ${ }^{93,135,232}$ the tissue fluid needs to be further divided into two compartments: the extra- and intrafibrillar compartments.

The development and assessment of the triphasic model depends on the determination of the material properties of the tissue. These material properties are parameters in the model. In some cases the material properties can be measured directly via experiments, and used as input for the model. Examples of these are the permeability coefficient for fluid flow and the diffusion coefficients of small solutes, such as ions.

Other material properties can not (easily) be determined directly from "xperinunts. These properties must be determined from model fits of results of carefully designr'd experiments. An example of such a material property is the solid compressive stressstrain relationship. It is difficult to determine this relationship directly, because of the presence of fluid in the tissue that influences the stiffness measurements of the solid.

The parameter that determines the viscous property of cartilaginous tissues is the permeability coefficient. The permeability coefficient is an important parameter not only for the viscous behaviour of the intervertebral disc, but also plays an important role in the nutrition of the disc. The disc is an avascular structure, and thus depends for it.s 
nutrition on the flow of water with its solutes into the disc. The rate of fluid flow through the disc depends directly on the permeability coefficient. The permeability coefficient thus also plays a role in the diurnal variation of disc height. In chapter 4 a method based on biphasic theory is used to determine the radial variation in the permeability coefficient for fluid flow in the radial direction of the annulus fibrosus of the canine IVD.

The separation of the elastic, viscous, and osmotic contributions in the mechanochemical behaviour of the annulus fibrosus of the IVD is undertaken in chapter 5 . In addition to the division of the material in solid, fluid and ions components, the fluid is divided between an extra- and intrafibrillar compartment. One-dimensional confined swelling and compression experiments serve as a basis for the model parameter determinations. From the equilibrium situations (Figure 1.7b) of these experiments estimates are made for the effective stress 8 versus the compressive strain of the samples, and the Donnan osmotic coefficients and activity coefficients. Estimates of the fluid content in the extrafibrillar and intrafibrillar compartments, and the concentration of fixed charges based on the extrafibrillar fluid volume are also made from these equilibrium situations. From the transients of the confined swelling and compression experiments the permeability and diffusion coefficients are estimated via fits of the experiments with the results of the one-dimensional computer implementation of the triphasic model.

BThe solid stress can be determined from the effective stress. 


\section{Chapter 2}

\section{Theory of triphasic mixtures}




\subsection{Introduction}

In models of the mechanical behaviour of swelling materials the most commonly used theory is that of mixtures. Mixture theory views upon the material as a mixture of $\nu$ constituents, that interact with each other. The interaction involves, e.g., mass transfer, momentum exchange, energy exchange. The theory of deforming porous media was originally developed by Terzaghi, ${ }^{210}$ and Biot. ${ }^{18,19}$ Later, Biot's theory was derived from the theory of mixtures by Bowen. ${ }^{25}$

The first mixture models of cartilage were biphasic: they involved a porous incompressible solid saturated with an incompressible fluid. ${ }^{145}$ Further development led to the triphasic model of swelling tissues, which accounts for osmotic swelling by means of the third or ion component ${ }^{\mathrm{a}}$, Lai et al., ${ }^{109}$ Snijders, ${ }^{200}$ and Snijders et al. ${ }^{203}$ The mixture of a solid, fluid and ions component is called a triphasic mixture, and the theory is referred to as triphasic (mixture) theory. Snijders et al. have developed a triphasic model with coupled differential equations based on the following degrees of freedom (dofs): the three solid displacements, $\boldsymbol{u}$, the fluid pressure, $p$, and the ions mass density $\rho^{ \pm}$.

In the general introduction of this thesis (sections 1.3.2 and 1.4.4) it is shown that the ions mass density, and fluid pressure are not continuous when the fixed charge density $\left(c^{p g}\right)$, or the fluid fraction $\left(\phi^{j}\right)$ are not continuous. In order to bypass numerical problems arising from discontinuities in the degrees of freedom, in this chapter a set of differential equations is derived, based on the chemical potentials of the ions and fluid, which are continuous when $c^{p s}$, or $\phi^{f}$ are not continuous.

In the first part of this chapter, the general mixture theory is outlined. The assumptions regarding the application of the mixture theory to cartilaginous tissue with three components are then given. Using the first and second laws of thermodynamics, the derivation of the differential equations for fluid flow and ions diffusion, based on the chemical potentials, is given. Finally, the degrees of freedom are introduced, on the basis of which the differential equations for momentum, fluid flow, and ions diffusion are written. The degrees of freedom are the three solid displacements $\boldsymbol{u}$, and two e-powers containing the chemical potentials for the ions, and the fluid respectively. The chemical potentials are not used directly, because that would lead to algebraically intricate expressions in the finite element formulation that is introduced in chapter 3 . This is in part overcome by using e-powers of the chemical potentials.

\footnotetext{
${ }^{a}$ Components are also sometimes unjustly called phases, a word usually reserved for the different states (gaseous, fluid, solid) one component can be in.
} 


\subsection{General mixture theory}

In this section the general mixture equations for a mixture with $\nu$ components are given.

\subsubsection{The mass balance}

The true mass density $\rho_{t}^{\alpha}$ for a component $\alpha$ in the mixture is given by:

$$
\rho_{t}^{\alpha}=\frac{m^{\alpha}}{V^{\alpha}}
$$

where $m^{\alpha}$ is the mass of component $\alpha$ and $V^{\alpha}$ is the volume taken by component $\alpha$ in the mixture. The local apparent mass density $\rho^{\alpha}$ is defined as follows:

$$
\rho^{\alpha}=\frac{m^{\alpha}}{V^{m}}
$$

where $m^{\alpha}$ represents the mass of component $\alpha$ in a representative mixture volume $V^{m}$. $V^{m}$ is large enough to be an averaging volume and small enough relative to the mixture volume. The volume fraction of component $\alpha$ is defined by:

$$
\phi^{a}=\frac{V^{\alpha}}{V^{m}} .
$$

From definitions (2.2) and (2.3) it is clear that:

$$
\sum_{\alpha=1}^{\nu} \phi^{\alpha}=1
$$

where $\nu$ is the number of components, and

$$
\rho^{\alpha}=\phi^{\alpha} \rho_{t}^{\alpha} .
$$

Equation (2.4) is the saturation constraint. The local form of the mass balance for component $\alpha$ is given by:

$$
\frac{\partial \rho^{\alpha}}{\partial t}+\nabla \cdot\left(\rho^{\alpha} v^{\alpha}\right)=\hat{c}^{\alpha},
$$

with:

$$
\begin{aligned}
& \frac{\partial \rho^{\alpha}}{\partial t}=\text { local time derivative of } \rho^{\alpha} \\
& \nabla=\text { Eulerian gradient operator, } \\
& \cdot=\text { dot vector (tensor) product operator, } \\
& v^{\alpha}=\text { velocity vector of component } \alpha, \\
& \hat{c}^{\alpha}=\text { mass of other components converted to mass of component } \alpha \text { per unit of volume } \\
& \text { and time. }
\end{aligned}
$$




\subsubsection{The balance of momentum}

The balance of momentum for the $\alpha$-th component is given by:

$$
\rho^{\alpha} \dot{\boldsymbol{v}}^{\alpha}=\boldsymbol{\nabla} \cdot \boldsymbol{\sigma}^{\alpha}+\rho^{\alpha} \boldsymbol{q}^{\alpha}+\hat{\boldsymbol{p}}^{\alpha},
$$

with:

$\dot{\boldsymbol{v}}^{\alpha}=$ time derivative of $\boldsymbol{v}^{\alpha}$, observed fixed to component $\alpha$, $\boldsymbol{\sigma}^{\alpha}=$ partial Cauchy stress tensor of component $\alpha$, $\boldsymbol{q}^{\alpha}=$ body force per unit mass of component $\alpha$,

$\hat{\boldsymbol{p}}^{\alpha}=$ momentum supply by other components for component $\alpha$.

\subsubsection{The balance of moment of momentum}

The balance of moment of momentum is given by:

$$
\hat{m}^{\alpha}-s^{\alpha}=0,
$$

with:

$\hat{\boldsymbol{m}}^{\alpha}=$ the moment of momentum supplied by other components, $\boldsymbol{s}^{\alpha}=$ axial vector of the skew symmetric tensor $\boldsymbol{\sigma}^{\alpha}-\left(\boldsymbol{\sigma}^{\alpha}\right)^{c}$.

\subsubsection{The balance of energy (first axiom of thermodynamics)}

The local form of conservation of energy for component $\alpha$ is given by (Truesdell and Toupin ${ }^{213}$ section 243 ):

$$
\rho^{\alpha} \hat{U}^{\alpha}=\rho^{\alpha} \tilde{r}^{\alpha}-\nabla \cdot \boldsymbol{h}^{\alpha}+\boldsymbol{\sigma}^{\alpha}: \boldsymbol{D}^{\alpha}+\hat{e}^{\alpha},
$$

with:

$\tilde{U}^{\alpha}=$ partial specific internal energy of component $\alpha$,

$\tilde{r}^{\alpha}=$ partial specific external heat supply other than from temperature gradients per unit of time for component $\alpha$,

$h^{\alpha}=$ partial heat llux vector for component $\alpha$,

$D^{\alpha}=\frac{1}{2}\left[\left(\Delta \boldsymbol{v}^{\alpha}\right)^{c}+\left(\Delta \boldsymbol{v}^{\alpha}\right)\right]=$ deformation rate tensor of component $\alpha$,

$\hat{e}^{\alpha}=$ heat supply per unit of time and volume for component $\alpha$ by other components.

The energy balance for the mixture is found when we add the energy terms from all possible interaction between the constituents (direct heat exchange $\left(\hat{e}^{\alpha}\right)$, monnentum supply $\left(\hat{\boldsymbol{p}}^{\alpha}\right)$, and mass exchange $\left.\left(\hat{c}^{\alpha}\right)\right)$. The sum of the energy exchange terms between the constituents must be zero:

$$
\sum_{\alpha}\left(\hat{e}^{\alpha}+\boldsymbol{w}^{\alpha} \cdot \hat{\boldsymbol{p}}^{\alpha}+\hat{\boldsymbol{c}}^{\alpha}\left(\tilde{U}^{\alpha}+\frac{1}{2} \boldsymbol{w}^{\alpha} \cdot \boldsymbol{w}^{\alpha}\right)\right)=0,
$$


with:

$$
\boldsymbol{w}^{\alpha}=\boldsymbol{v}^{\alpha}-\boldsymbol{v}^{m}
$$

$\boldsymbol{w}^{\alpha}$ is the difference between the component velocity $\boldsymbol{v}^{\alpha}$ and the mixture velocity $\boldsymbol{v}^{m}$, which is defined as:

$$
v^{m}=\frac{1}{\rho^{m}} \sum_{\alpha} \rho^{\alpha} v^{\alpha}
$$

where $\rho^{m}=\sum_{\alpha} \rho^{\alpha}$ is the mass density of the mixture, and the summation is over the components of the mixture.

\subsubsection{The entropy inequality (second axiom of thermodynam- ics)}

The second law of thermodynamics (the entropy inequality) states that the cntropy of a. system can only increase or stay the same. We apply the second law to a representative volume of the mixture. This implies that the entropy change of the different components: of the mixture can increase or decrease, as long as the entropy change of the total mixture is greater than or equal to zero. For each component $\alpha$ it is hypothesized that the entropy production per unit of volume and time, $\xi^{\alpha}$, equals: ${ }^{25}$

$$
\xi^{\alpha}=\rho^{\alpha} \dot{S}^{\alpha}+\nabla \cdot\left(\frac{\boldsymbol{h}^{\alpha}}{T^{\alpha}}\right)-\frac{\rho^{\alpha} \tilde{r}^{\alpha}}{T^{\alpha}}+\hat{c}^{\alpha} \tilde{S}^{\alpha},
$$

with:

$\tilde{S}^{\alpha}=$ specific entropy of component $\alpha$, $T^{\alpha}=$ absolute temperature of component $\alpha$.

The entropy production $\xi^{\alpha}$ consists of a specific entropy term, $\rho^{\alpha} \overline{\tilde{S}}^{\alpha}$, a term associated with heat flux due to temperature gradients, $\nabla \cdot\left(\frac{h^{a}}{T^{a}}\right)$, a term due to heat supply other than from temperature gradients, $\frac{p^{a} r^{\alpha}}{T^{\alpha}}$, and a term related to mass supply from other constituents, $\hat{c}^{\alpha} \tilde{S}^{\alpha}$.

\subsection{The three component mixture as a model for car- tilaginous tissues}

The class of problems in cartilage mechanics this thesis considers comprises quasi-static compression, extension, rotation and bending of a mixture of a solid, fluid and uniunivalent salt, such as $\mathrm{Na}$ ('l. Swelling of these mixtures as a result of fluid and osmotic pressure gradients is an essential feature of triphasic mixture models. Relative fluid flow due to fluid and osmotic pressure gradients as well as diffusion of small ions are both important in the mechanics of charged swelling materials. Donnan osmosis is chosen as the model for material swelling. ${ }^{11,33,50,203}$ Relative fluid flow is governed by the gradient in fuid pressure $p$ minus the osmotic pressure $\pi, p-\pi$. Internal electrical fields that are a consequence of fluid flow, or ion diffusion (streaming potential, diffusion potential ${ }^{74,92,125}$ ) 
are not accounted for in the triphasic theory. A Donnan electrical potential difference is defined across the boundary. This potential difference, however, does not influence ions diffusion nor fluid flow, because ions are assumed to travel in neutral pairs of salt molecules.

\subsubsection{Assumptions regarding the three component mixture of cartilaginous tissues}

The three component mixture consists of a solid (superscript $s$ ), a fluid $(f$ ) and ions (univalent salt; \pm ). The superscript $\alpha$ stands for $s, f$, and \pm , the superscript $\beta$ stands for $f$ and \pm . Summation over $\alpha$ means summation over $s, f$, and \pm . Summation over $\beta$ means summation over $f$ and \pm .

Compressibility of components, temperature, the ions component

- The components are assumed to be intrinsically incompressible.

- The absolute temperature of the components is assumed constant in time and place:

$$
\begin{gathered}
T^{s}=T^{f}=T^{ \pm}, \\
\dot{T}^{\alpha}=0, \\
\nabla T^{\alpha}=\mathbf{0} .
\end{gathered}
$$

- We restrict the scope of the model to an ions component that cursists of a monovalent salt, for which we will take $\mathrm{NaCl}$, as this is the most common monovalent salt in biological tissues. We neglect the phenomenon of counterion condensation, ${ }^{74}$ i.e., the chemical binding of ions to the fixed charges, which primarily occurs with multivalent cations, such as $\mathrm{Ca}^{2+}$.

The amounts of the separate ions are often expressed as a function of their concentration per unit fluid volume $\left(c^{+}\right.$and $\left.c^{-}\right)$. We define the velocity of the salt as the mass average of the velocity of the ions:

$$
\begin{aligned}
\rho^{+}=M^{+} \phi^{f} c^{+} ; \quad & \rho^{-}=M^{-} \phi^{\jmath} c^{-}, \\
& \rho^{ \pm}=\rho^{+}+\rho^{-}, \\
\rho^{ \pm} v^{ \pm}= & \rho^{+} v^{+}+\rho^{-} v^{-},
\end{aligned}
$$

with:

$M^{+}, M^{-}=$the molar masses of positive and negative ions,

$v^{ \pm}=$the velocity vector of the salt,

$v^{+}=$the velocity vector of the cation,

$v^{-}=$the velocity vector of the anion.

The volume fraction of the ions is neglected compared to those of the solid and the fluid (dilute solution).

$$
\phi^{s}+\phi^{f}+\phi^{\sharp} \approx \phi^{s}+\phi^{f}=1 \text {. }
$$


The maximum volume fraction of the ions for a $0.6 \mathrm{M} \mathrm{NaCl}$ solution is approximately 0.008 in pure water.

The mass balance The mass of other components converted to mass of component $\alpha$, is assumed to be zero:

$$
\hat{c}^{\alpha}=0
$$

This means that there are no chemical reactions between the components. We further assume that the true densities are constant, i.e., not dependent of composition and pressure:

$$
\rho_{t}^{\alpha}=\text { constant. }
$$

This implies that partial molar volumes in the mixture equal molar volumes of the unmixed component.

It follows from (2.5), (2.19), and (2.20), that we can write for the local mass balances of the components (2.6):

$$
\frac{\partial \phi^{\alpha}}{\partial t}+\nabla \cdot\left(\phi^{\alpha} v^{\alpha}\right)=0
$$

Summation of the mass balances (2.21) and eliminating the local time derivatives leads to the mass balance of the mixture:

$$
\sum_{\alpha} \nabla \cdot\left(\phi^{\alpha} v^{\alpha}\right)=0
$$

On account of (2.18), we neglect the term with $\phi^{ \pm}$in (2.22), and get for the mass balance of the mixture:

$$
\nabla \cdot\left(\phi^{s} v^{s}\right)+\nabla \cdot\left(\phi^{f} v^{f}\right)=0
$$

which can also be written as:

$$
\nabla \cdot v^{s}+\nabla \cdot\left(\phi^{f}\left(v^{f}-v^{s}\right)\right)=0
$$

The balance of momentum Momentum is assumed to be conserved for the total mixture, so the sum of the momentum supplies from the components $\alpha$ equals 0 :

$$
\sum_{\alpha} \hat{p}^{\alpha}=0
$$

We only consider situations with negligible accelerations, and neglect the inertial term $\rho^{\alpha} \dot{v}^{\alpha}$ in (2.7). Body forces $\left(q^{\alpha}\right)$ from, e.g., gravitational and electromagnetic fields are also neglected. Because of these assumptions (2.7) results into:

$$
\boldsymbol{\nabla} \cdot \boldsymbol{\sigma}^{\alpha}+\hat{\boldsymbol{p}}^{\alpha}=\mathbf{0}
$$

A summation over the three components in (2.26) and using (2.25) leads to:

$$
\nabla \cdot \sigma^{s}+\nabla \cdot \sigma^{f}+\nabla \cdot \sigma^{ \pm}=0
$$

Equation (2.27) is the basis for the momentum differential equation, as is shown later. 
The balance of moment of momentum We assume that the moment of momentum supply by other components equals 0 . This implies that the partial Cauchy stress tensor of component $\alpha$ is symmetrical, and means physically that there is no exchange of rotational energy between the components, which is acceptable in media where pores intercommunicate in a random way:

$$
\sigma^{\alpha}=\left(\sigma^{\alpha}\right)^{c}
$$

The entropy inequality According to (2.19) we can leave out the term with $\hat{c}^{\alpha}$ in the entropy inequality (2.13). The entropy change of the total mixture is assumed to be greater than or equal to zero, thus the sum of entropy productions of the separate components must be positive or zero:

$$
\sum_{\alpha} \xi^{\alpha}=\sum_{\alpha}\left(\rho^{\alpha} \tilde{\tilde{S}}^{\alpha}+\nabla \cdot\left(\frac{\boldsymbol{h}^{\alpha}}{T^{\alpha}}\right)-\frac{\rho^{\alpha} \tilde{r}^{\alpha}}{T^{\alpha}}\right) \geq 0 .
$$

\subsubsection{Combining the first and second laws of thermodynamics to derive the differential equations for fluid flow and ion diffusion}

In this section we combine the first law of thermodynamics (conservation of energy) with the second law (entropy inequality). The result is an inequality that can only be satisfied when certain terms in this inequality equal zero. The inequality that remains after elimination of the terms that are zero, is satisfied by introducing $\boldsymbol{B}$ tensors, which are semi-positive definite. The equations resulting from the introduction of the $\boldsymbol{B}$ tensors (with chemical potentials) are the basis for the differential equations for ions diffusion and fluid flow.

We define the specific chemical potential tensor $\tilde{M}^{\alpha}$ for component $\alpha$ as: ${ }^{24}$

$$
\rho^{\alpha} \tilde{\boldsymbol{M}}^{\alpha}=\rho^{\alpha} \tilde{F}^{\alpha} \boldsymbol{I}-\boldsymbol{\sigma}^{\alpha}, \mathrm{b}
$$

with:

$$
\tilde{F}^{\alpha}=\tilde{U}^{\alpha}-T^{\alpha} \tilde{S}^{\alpha}
$$

the Helmholz free energy per unit mass of component $\alpha$.

Combining the energy balance equations (2.9) and (2.10), and the entropy inequality (2.29), with restrictions (2.14)-(2.16), (2.19), and using (2.30) and (2.31) leads to:

$$
\sum_{\alpha}\left(-\dot{F} \alpha-\rho^{\alpha} \tilde{\boldsymbol{M}}^{\alpha}: D^{\alpha}-\hat{\boldsymbol{p}}^{\alpha} \cdot \boldsymbol{w}^{\alpha}\right) \geq 0 \text {, }
$$

with:

$$
\check{F}^{\alpha}=\rho^{\alpha} \check{F}^{\alpha},
$$

${ }^{b}$ It is possible to write the chemical potential tensor as a scalar, which is later identified with the familiar form of equation (1.10). Note that the chemical potential tensor introduced here, is defined per unit of mass (specific), while the chemical potential from eq. (1.10) is defined as a molar quantity. Conversion of one form to the other is easily done by dividing the molar chemical potential by the molar mass. 
the Helmholz free energy per unit of mixture volume for component $\alpha$.

With regards to the derivation of (2.32) we remark that the energy balance for the mixture (2.10) was used for the elimination of $\hat{e}^{\alpha},(2.31)$ was used for the elimination of $\tilde{U}^{\alpha},(2.33)$ was used for the elimination of $\tilde{F}^{\alpha}$, and the mass balance (2.6) with $\hat{c}^{\alpha}=0$ is responsible for the elimination of the term $\rho^{\alpha} \tilde{F}^{\alpha} \boldsymbol{I}: \boldsymbol{D}^{\alpha}=\rho^{\alpha} \tilde{F}^{\alpha} \boldsymbol{\nabla} \cdot \boldsymbol{v}^{\alpha}$, that arises from the introduction of the chemical potential tensor (2.30).

To account for the mass balance of the mixture (2.22), we put it, multiplied by a Lagrange multiplier $\lambda$, in the inequality (2.32) and get:

$$
\sum_{\alpha}\left(-\check{F}^{\alpha}-\rho^{\alpha} \check{M}^{\alpha}: D^{\alpha}-\hat{\boldsymbol{p}}^{\alpha} \cdot \boldsymbol{w}^{\alpha}+\lambda \nabla \cdot\left(\phi^{\alpha} \boldsymbol{v}^{\alpha}\right)\right) \geq 0
$$

Making use of the saturation constraint (2.4) and the demand of conservation of momentum for the mixture (2.25) we rewrite (2.34) as:

$$
\begin{aligned}
& \sum_{\alpha}\left(-\dot{F}^{\alpha}-\left(\rho^{\alpha} \tilde{\boldsymbol{M}}^{\alpha}-\lambda \phi^{\alpha} \boldsymbol{I}\right): D^{\alpha}\right)- \\
& \sum_{\beta}\left(\hat{\boldsymbol{p}}^{\beta}-\lambda \nabla \phi^{\beta}\right) \cdot\left(\boldsymbol{v}^{\beta}-\boldsymbol{v}^{o}\right) \geq 0 .
\end{aligned}
$$

To satisfy (2.35) we first have to choose a set of independent variables as a function of which we can write the inequality. Because of the demand of frame indifference for the constitutive equations, we use Lagrangian forms. This choice ensures that rigid body rotations of the medium do not alter its properties. Within these restrictions there are a number of possibilities to choose a set from. Considerations for a certain choice come from experimental data and other practical matters such as how complex the model is to be. The above restrictions and reasoning resulted in the following set of independent variables ${ }^{c}$ :

$$
\boldsymbol{E}^{s}, \phi^{\beta}, \boldsymbol{v}^{* \beta} ; \quad \beta=f_{2} \pm
$$

with:

$$
\boldsymbol{v}^{* \beta}=\boldsymbol{F}^{-1} \cdot\left(\boldsymbol{v}^{\beta}-\boldsymbol{v}^{s}\right)^{\mathrm{d}},
$$

the Lagrangian velocity difference between the fluid or ions and the sulid, and: $F^{-1}=$ the inverse of the deformation gradient tensor of the mixture or solid, $\boldsymbol{E}^{s}=$ Green-Lagrange strain tensor of the solid.

For the dependent variables of (2.35) we define:

$$
\begin{aligned}
\check{F}^{\alpha} & =\dot{F}^{\alpha}\left(\boldsymbol{E}^{s}, \phi^{\beta}, \boldsymbol{v}^{* \beta}\right), \\
\rho^{\alpha} \tilde{\boldsymbol{M}}^{\alpha}-\lambda \dot{\phi}^{\alpha} \boldsymbol{I} & =\boldsymbol{F} \cdot \tilde{\boldsymbol{M}}^{* \alpha}\left(\boldsymbol{E}^{s}, \phi^{\beta}, \boldsymbol{v}^{* \beta}\right) \cdot \boldsymbol{F}^{c}, \\
\hat{\boldsymbol{p}}^{f}-\lambda \nabla \phi^{J} & =\boldsymbol{F} \cdot \hat{\boldsymbol{p}}^{-J}\left(\boldsymbol{E}^{s}, \phi^{\beta}, \boldsymbol{v}^{-\beta}\right), \\
\hat{\boldsymbol{p}}^{ \pm}-\lambda \nabla \phi^{ \pm} & =\boldsymbol{F} \cdot \hat{\boldsymbol{p}}^{* \pm}\left(\boldsymbol{E}^{s}, \phi^{\beta}, \boldsymbol{v}^{* \beta}\right) .
\end{aligned}
$$

Note that the independent variables do not equal the degrees of freedom.

Note that the Lagrangian velocity difference is an independent variable, and that (2.37) does not serve to indicate a dependence of $v^{* \beta}$ on $\boldsymbol{F}^{-1}$, or $\boldsymbol{v}^{\alpha}$. It merely gives the relationship between Lagrangian and Eulerian variables. 
The terms with an asterisk are Lagrangian forms, those without are Eulerian forms. ${ }^{e}$ Conversion from Eulerian to Lagrangian forms, and back transformation is done using:

$$
\begin{array}{r}
b=\boldsymbol{F} \cdot \boldsymbol{a}^{*} ; \quad a^{*}=\boldsymbol{F}^{-1} \cdot \boldsymbol{b}, \\
\boldsymbol{B}=\boldsymbol{F} \cdot \boldsymbol{A}^{*} \cdot \boldsymbol{F}^{c} ; \quad A^{*}=\boldsymbol{F}^{-1} \cdot \boldsymbol{B} \cdot \boldsymbol{F}^{-c},
\end{array}
$$

where $\boldsymbol{b}, \boldsymbol{B}$ are a Eulerian vector, respectively tensor, and $\boldsymbol{a}^{*}$ and $\boldsymbol{A}^{*}$ are Lagrangian forms. Because of the demand of equipresence, all the dependent variables are functions of the complete set of independent variables.

When we substitute (2.38) in (2.35), using the chain rule of differentiation to write the derivatives of functions on the right hand side of (2.38) as functions of the independent variables, we get:

$$
\begin{aligned}
& -\left[\boldsymbol{F} \cdot\left(\tilde{\boldsymbol{M}}^{* s}+\frac{\partial \check{F}}{\partial \boldsymbol{E}^{s}}\right) \cdot \boldsymbol{F}^{c}\right]: \boldsymbol{D}^{s} \\
& -\sum_{\boldsymbol{l}}\left[\boldsymbol{F} \cdot \tilde{\boldsymbol{M}}^{* \beta} \cdot \boldsymbol{F}^{c}-\phi^{\beta} \frac{\partial \check{F}}{\partial \phi^{\beta}} \boldsymbol{I}\right]: \boldsymbol{D}^{\beta} \\
& -\sum_{\beta} \frac{\partial \check{F}}{\partial \boldsymbol{v}^{* \hat{\beta}}} \cdot\left(\boldsymbol{v}^{* \beta}\right), \\
& +\left[-\boldsymbol{F} \cdot \dot{\boldsymbol{p}}^{* \pm}-\nabla \check{F}^{ \pm}+\frac{\partial \check{F}}{\partial \phi^{ \pm}} \nabla \phi^{ \pm}\right] \cdot\left(\boldsymbol{v}^{ \pm}-\boldsymbol{v}^{f}\right) \\
& +\left[-\boldsymbol{F} \cdot\left(\dot{\boldsymbol{p}}^{* f}+\hat{\boldsymbol{p}}^{* \pm}\right)-\nabla \check{F}^{f}-\nabla \check{F}^{ \pm}\right. \\
& \left.+\frac{\partial \check{F}}{\partial \phi^{\prime}} \nabla \phi^{f}+\frac{\partial \check{F}}{\partial \phi^{ \pm}} \nabla \phi^{ \pm}\right] \cdot\left(\boldsymbol{v}^{f}-\boldsymbol{v}^{s}\right) \geq 0,
\end{aligned}
$$

with:

$$
\dot{F}=\check{F}^{*}+\check{F}^{\prime}+\check{F}^{ \pm}
$$

the Helmholz free energy of the mixture per unit of mixture volume, and:

$$
\left(v^{* \beta}\right)_{s}=\frac{\partial v^{* i+3}}{\partial t}+v^{s} \cdot \nabla v^{* ;}
$$

the variation of $v^{* \beta}$ in time, observed fixed to the solid component.

The inequality $(2.40)$ has to hold for any values of $D^{\alpha}, \boldsymbol{v}^{* \beta}$, and $\left(\boldsymbol{v}^{-\beta}\right)_{s}$. Therefore. because the terms in front of the $D^{\alpha}$ and $\left(v^{* 3}\right)_{s}$ are not functions of these velocity gradients and time derivative's, these terms must be set to zero in order to comply with the inequality:

$$
\begin{gathered}
\tilde{\boldsymbol{M}}^{* s}=-\frac{\partial \check{F}}{\partial \overline{\boldsymbol{E}^{s}}}, \\
\boldsymbol{F} \cdot \tilde{\boldsymbol{M}}^{* \beta} \cdot \boldsymbol{F}^{c}=\phi^{\beta} \frac{\partial \check{F}}{\partial \phi^{\beta}} \boldsymbol{I},
\end{gathered}
$$

"Note that the Lagrangian forms have been conveniently converted from the Eulerian forms used in (2.35), implying that the symbol $\boldsymbol{M}^{* a}$ does not stand for the Lagrangian chemical potential tensor, but the Lagrangian conversion of the sum of the mass density $\rho^{\alpha}$ times the Eulerian $M^{\alpha}$ and the term $-\lambda \phi^{\circ} I$. The same reasoning applies to the $\hat{p}$ terms. 


$$
\frac{\partial \check{F}}{\partial \boldsymbol{v}^{* \beta 3}}=\mathbf{0}
$$

From (2.44) we deduce that the chemical potentials of the fluid and ions can be written as scalars. Writing these as $\tilde{\mu}^{f}$ and $\tilde{\mu}^{ \pm}$respectively:

$$
\begin{gathered}
\tilde{\mu}^{f}=\frac{1}{\rho_{t}^{f}}\left(\frac{\partial \check{F}}{\partial \phi^{\prime}}+\lambda\right), \\
\tilde{\mu}^{ \pm}=\frac{1}{\rho_{t}^{ \pm}}\left(\frac{\partial \check{F}}{\partial \phi^{ \pm}}+\lambda\right) .
\end{gathered}
$$

Another important conclusion can be drawn from (2.45): the Helmholz free energy of the mixture does not depend on the relative velocities of the components.

We now rewrite the remaining inequality:

$$
\begin{aligned}
& {\left[-\boldsymbol{F}^{c} \cdot \boldsymbol{F} \cdot \hat{\boldsymbol{p}}^{* \pm}-\nabla_{0} \check{F}^{ \pm}+\frac{\partial \check{F}}{\partial \phi^{ \pm}} \nabla_{0} \phi^{ \pm}\right] \cdot\left(\boldsymbol{v}^{* \pm}-\boldsymbol{v}^{* f}\right)} \\
& +\left[-\boldsymbol{F}^{c} \cdot \boldsymbol{F} \cdot\left(\hat{\boldsymbol{p}}^{* f}+\hat{\boldsymbol{p}}^{* \pm}\right)-\nabla_{0} \check{F}^{f}-\nabla_{0} \check{F}^{ \pm}\right. \\
& \left.\left.+\frac{\partial \check{F}}{\partial \phi^{f}} \nabla_{0} \phi^{f}+\frac{\partial \check{F}}{\partial \phi^{ \pm}} \nabla_{0} \phi^{ \pm}\right] \cdot \boldsymbol{v}^{* f}\right) \geq 0
\end{aligned}
$$

where we have used the Lagrangian gradients with respect to the reference configuration "0":

$$
\boldsymbol{\nabla}=\boldsymbol{F}^{-\varepsilon} \cdot \nabla_{0}
$$

The terms in (2.48) are not linear in the velocities $v^{* \pm}$ and $v^{* f}$, because the momentum interaction terms $\left(\hat{\boldsymbol{p}}^{*}\right)$ are functions of these velocities. Therefore, the terms by which the velocity differences are multiplied, cannot be set to zero. We salisfy the inequality (2.48) by demanding that:

$$
\begin{gathered}
\boldsymbol{F}^{c} \cdot \boldsymbol{F} \cdot \hat{\boldsymbol{p}}^{* \pm}+\nabla_{0} \check{F}^{ \pm}-\frac{\partial \check{F}}{\partial \phi^{ \pm}} \nabla_{0} \phi^{ \pm}=-\boldsymbol{B}^{f \pm} \cdot\left(\boldsymbol{v}^{* \pm}-\boldsymbol{v}^{* f}\right), \\
\boldsymbol{F}^{c} \cdot \boldsymbol{F} \cdot\left(\hat{\boldsymbol{p}}^{* f}+\hat{\boldsymbol{p}}^{* \pm}\right)+\nabla_{0} \check{F}^{f}+\nabla_{0} \check{F}^{ \pm} \\
-\frac{\partial \check{F}}{\partial \phi^{f}} \nabla_{0} \phi^{f}-\frac{\partial \dot{F}}{\partial \phi^{ \pm}} \nabla_{0} \phi^{ \pm}=-\boldsymbol{B}^{s f} \cdot\left(\boldsymbol{v}^{* f}-\boldsymbol{v}^{* s}\right),
\end{gathered}
$$

where $B^{s f}$ and $B^{J \pm}$ are semi-positive definite tensors, and $\boldsymbol{v}^{* s}(=0)$ has been added for the sake of uniform notation.

We can now find relations that link the gradients of the chemical potentials to the Lagrangian velocity differences multiplied by the $B$ tensors. We use (2.26), (2.30), (2.46) - (2.51) to find:

$$
\rho^{ \pm} \nabla_{0} \bar{\mu}^{ \pm}=-B^{f \pm} \cdot\left(v^{* \pm}-v^{* f}\right),
$$

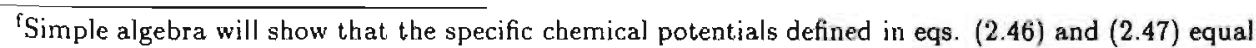
the molar chemical potential of eqs. $(1.10)$ and (1.11), divided by the molar mass. The $\lambda$ will later be identified with the fluid pressure $p$, and the term with derivative of the Helmholz free energy of the mixture equals the expression in (1.12) divided by the molar mass. 


$$
\rho^{f} \nabla_{0} \tilde{\mu}^{f}=-B^{s f} \cdot \boldsymbol{v}^{* f}+B^{f \pm} \cdot\left(\boldsymbol{v}^{* \pm}-\boldsymbol{v}^{* f}\right) .
$$

Adding (2.52) and (2.53) leads to the extended Darcy equation:

$$
\boldsymbol{v}^{* f}=-\left(\boldsymbol{B}^{s f}\right)^{-1} \cdot\left(\rho^{ \pm} \nabla_{0} \tilde{\mu}^{ \pm}+\rho^{f} \nabla_{0} \tilde{\mu}^{f}\right) .
$$

In (2.54) we see that fluid flow relative to the solid is caused by gradients in the chemical potentials of the ions and the fluid.

\subsubsection{Constitutive behaviour; the Cauchy stresses, fluid pres- sure, strain energy function}

The constitutive relationships for the partial Cauchy stresses are determined from (2.30), (2.38), (2.43), and (2.44):

$$
\begin{gathered}
\boldsymbol{\sigma}^{s}=\left(\check{F}^{s}-\lambda \phi^{s}\right) \boldsymbol{I}+\boldsymbol{F} \cdot \frac{\partial \check{F}}{\partial \boldsymbol{E}^{s}} \cdot \boldsymbol{F}^{c} \\
\boldsymbol{\sigma}^{\prime}=\left(\check{F}^{f}-\lambda \phi^{\prime}-\phi^{f} \frac{\partial \check{F}}{\partial \phi^{f}}\right) \boldsymbol{I}, \\
\boldsymbol{\sigma}^{ \pm}=\left(\check{F}^{ \pm}-\lambda \phi^{ \pm}-\phi^{ \pm} \frac{\partial \dot{F}}{\partial \phi^{ \pm}}\right) \boldsymbol{I} .
\end{gathered}
$$

From (2.56) and (2.57) the partial stresses of the fluid and ions can be written as scalars. The sum of the three partial stresses yields the total stress $\sigma$, which can be written as the sum of an effective stress, $\sigma_{e}$, and the negative of the fluid pressure $-p$ times the unit tensor $\boldsymbol{I}$ :

$$
\boldsymbol{\sigma}^{s}+\boldsymbol{\sigma}^{\jmath}+\boldsymbol{\sigma}^{ \pm}=\boldsymbol{\sigma}_{e}-p \boldsymbol{I}
$$

From (2.55) - (2.58), the Lagrange multiplier $\lambda$ can be interpreted as the fluid pressure $p$.

Mechanical loads are supported by the effective stress and fluid pressure. The boundary condition for the momentum of the mixture is:

$$
\left(\sigma_{e}-p I\right) \cdot n=t
$$

with:

$\boldsymbol{n}=$ the unit normal vector on the boundary,

$t=$ the boundary stress vector.

To find a relationship between the strain energy function $\psi$ and the effective stress, we use the Lagrangian forms of the dependent and independent variables. Thus, we write the strain energy function as the free energy per unit of reference volume:

$$
\psi\left(\boldsymbol{E}^{s}, \phi^{\jmath} \operatorname{det} \boldsymbol{F}, \phi^{ \pm} \operatorname{det} \boldsymbol{F}\right)=\dot{F}\left(\boldsymbol{E}^{s}, \phi^{f}, \phi^{ \pm}\right) \operatorname{det} \boldsymbol{F} .
$$

Using $(2.55)-(2.60)$ one can show:

$$
\boldsymbol{\sigma}_{\varepsilon}=(\operatorname{det} \boldsymbol{F})^{-1} \boldsymbol{F} \cdot \frac{\partial \psi}{\partial \boldsymbol{E}^{5}} \cdot \boldsymbol{F}^{c} .
$$




\subsection{The differential equations based on chemical po- tentials}

\subsubsection{The degrees of freedom}

The driving forces for fluid flow and ion diffusion in a porous charged swelling material are proportional to the gradients of their respective chemical potentials. ${ }^{74,179}$ These chemical potentials are continuous at boundaries with pressure and concentration jumps. For this reason expressions with the chemical potentials were chosen as degrees of freedom for the present formulation.

We use specific chemical potentials in the numerical formulation, and thus divide the molar chemical potentials by the molar masses. Using (1.13), we get for the specific chemical potential of the salt, $\tilde{\mu}^{ \pm}$:

$$
\tilde{\mu}^{ \pm}=\tilde{\mu}_{0}^{ \pm}+\frac{R T}{M^{ \pm}} \ln \left(\left(\gamma^{ \pm}\right)^{2} x^{+} x^{-}\right),
$$

with:

$\tilde{\mu}_{0}^{ \pm}=$the standard specific chemical potential of the salt, $M^{ \pm}=M^{+}+M^{-}=$molar mass of the salt.

In the situation of an ion exchange material with fixed charges, the molar fraction of the cations exceeds that of the anions, because a part of the positive ions is needed to neutralize the fixed negative charges. Normally, eq. (2.62) is used for the chemical potential of the neutral salt. We have a neutral salt outside the material. However, inside the swelling material, (2.62) is used as an approximation of the chemical potential of all ions, positive and negative. From (1.14) we get for the specific chemical potential $\tilde{\mu}^{f}$ of the fluid:

$$
\tilde{\mu}^{f}=\tilde{\mu}_{0}^{f}+\frac{R T}{M^{\prime}} \ln \left(\gamma^{f} x^{f}\right)+p \tilde{V}^{\prime},
$$

with:

$\tilde{\mu}_{0}^{f}=$ the standard specific chemical potential of the fluicl,

$M^{f}=$ molar mass of the fluid,

$\tilde{V}^{f}=1 / \rho_{t}^{f}=$ partial specific volume of the fluid. ${ }^{\mathrm{g}}$

Because the chemical potentials contain logarithmic terms, which make the formulation algebraically complex, we opted for forms of the chemical potentials with e-powers, thus eliminating the logarithmic term in $\tilde{\mu}^{ \pm}$:

$$
\begin{aligned}
& \epsilon_{c}=e^{\frac{\tilde{p}^{\mathbf{t}}-\tilde{\mu}_{0}^{ \pm}}{\tilde{R} T} M^{ \pm}}, \\
& \epsilon_{p}=e^{\frac{\hat{p}^{\prime} / \tilde{\mu}_{0}^{\prime}}{R T} M^{\prime}} .
\end{aligned}
$$

${ }^{8}$ Note that in agreement with the assumption of constant true densities, the partial specific volume of the fluid is assumed to be constant and equal to the specific volume $1 / p_{6}$. 
Thus, the degrees of freedom (dofs) used in the present formulation are $\boldsymbol{u}\left(u_{1}, u_{2}, u_{3}\right)$, the solid displacements, $\epsilon_{p}$, a function of the chemical potential of the fluid, and $\epsilon_{\varepsilon}$, a function of the chemical potential of the salt (ions).

To formulate the differential equations in terms of $\epsilon_{p}$ and $\epsilon_{c}$, we need to write the negative ion concentration $c^{-}$, the pressure $\mathrm{p}$, and the ion density $\rho^{ \pm}$in terms of them. For this, we use the dilute solution approximations for the molar fractions (1.16) and (1.17), and the demands of bulk electroneutrality (1.18). From (2.62) and (2.64), with approximations (1.17), and using (1.18), $\epsilon_{c}$ can be written as:

$$
\epsilon_{c}=\left(\frac{\gamma^{ \pm}}{c^{f}}\right)^{2} c^{+} c^{-}=\left(\frac{\gamma^{ \pm}}{c^{f}}\right)^{2}\left(c^{-}+c^{p g}\right) c^{-} .
$$

From (2.66) we write $c^{-}$as a function of $\epsilon_{c}$ and $c^{p g}$ :

$$
c^{-}=\frac{1}{2}\left(-c^{p g}+\sqrt{\left(c^{p g}\right)^{2}+\left(\frac{2 c^{f}}{\gamma^{ \pm}}\right)^{2} \epsilon_{c}}\right) .
$$

For ease of notation we abbreviate some terms:

$$
\begin{gathered}
c_{ \pm}^{f}:=\frac{2 c^{J}}{\gamma^{ \pm}}, \\
c^{\kappa}:=\sqrt{\left(c^{p g}\right)^{2}+\left(\frac{2 c^{f}}{\gamma^{ \pm}}\right)^{2} \epsilon_{c} .}
\end{gathered}
$$

From (1.16), (2.63), (2.65), (2.67), and (2.69) we write for the fluid pressure $p$ as a function of $\epsilon_{p}, \epsilon_{c}$, and $c^{p g}$ :

$$
p=c^{J} R T\left[\ln \epsilon_{p}-\ln \gamma^{J} x^{J}\right]=c^{J} R T \ln \left[\frac{\epsilon_{p}}{\gamma^{f}}\left(1+\frac{c^{\kappa}}{c^{f}}\right)\right]
$$

From (1.18), (2.17), (2.67), and (2.69) we write $\rho^{ \pm}$as a function of $\epsilon_{c}, c^{p g}$, and $\phi^{f}$ :

$$
\rho^{ \pm}=\frac{1}{2} \phi^{J}\left[M^{d i f} c^{p g}+M^{ \pm} c^{\kappa}\right]
$$

with:

$M^{\text {dif }}=M^{+}-M^{-}$.

Note that we write $c^{-}, p$, and $\rho^{ \pm}$as functions of $c^{p g}$, whilst $c^{p g}$ is not a dof. It is a function of the deformation and therefore a function of the dofs $u_{i}$. This is done for simplicity of notation.

The gradients of $p$ and $\rho^{ \pm}$for the reference state indicated with a " $0^{\prime \prime}$ are given by:

$$
\begin{aligned}
& \nabla_{\mathrm{o}} p=c^{J} R T\left[\frac{\boldsymbol{\nabla}_{0} \epsilon_{p}}{\epsilon_{p}}+\Phi^{f} \frac{c^{p g} \nabla_{0} c^{p g}+\frac{1}{2}\left(c_{ \pm}^{f}\right)^{2} \nabla_{0} \epsilon_{c}}{c^{\kappa}\left(c^{f}+c^{\kappa}\right)}\right], \\
& \boldsymbol{\nabla}_{\mathrm{O} \rho^{ \pm}}=\frac{1}{2}\left[M^{d i f} c^{p g}+M^{ \pm} c^{\kappa}\right] \nabla_{0} \phi^{f} \\
& \quad+\frac{1}{2} \phi^{J}\left[\left(M^{d i f}+\frac{M^{ \pm} c_{c}^{p g}}{c^{\kappa}}\right) \nabla_{0} c^{p g}+M^{ \pm} \frac{\frac{1}{2}\left(c_{ \pm}^{f}\right)^{2} \nabla_{0} \epsilon_{c}}{c^{\epsilon}}\right] .
\end{aligned}
$$


In $(2.72) \Phi^{f}$ is defined by:

$$
\Phi^{f}=1+\frac{\partial \ln \gamma^{f}}{\partial \ln x^{f}}
$$

The gradients of $\epsilon_{p}$ and $\epsilon_{c}$ are given by:

$$
\begin{aligned}
& \nabla_{0} \epsilon_{c}=\epsilon_{c} \frac{M^{ \pm}}{R T} \nabla_{0} \tilde{\mu}^{ \pm}, \\
& \nabla_{0} \epsilon_{p}=\epsilon_{p} \frac{M^{f}}{R T} \nabla_{0} \tilde{\mu}^{f} .
\end{aligned}
$$

Substituting (1.16) into (2.63), and (1.17) into (2.62) we find for a dilute solution:

$$
\begin{aligned}
& \nabla_{0} \tilde{\mu}^{ \pm}=\nabla_{0}\left(c^{+} c^{-}\right), \\
& \nabla_{0} \tilde{\mu}^{\prime}=\nabla_{0}(p-\pi) .
\end{aligned}
$$

In $(2.78) \pi$ is the Donnan osmotic pressure:

$$
\pi \approx R T\left(c^{+}+c^{-}\right), \quad \text { or } \quad \pi=\Phi R T\left(c^{+}+c^{-}\right),
$$

with $\Phi$ the osmotic coefficient $(0<\Phi \leq 1)$. For $(2.79)$ approximations $\ln (1 / 1-x) \approx$ $\ln (1+x) \approx x$ were used, and both $\gamma^{f}$ and $\gamma^{ \pm}$were assumed constant for (2.78) and (2.77) respectively.

\subsubsection{The momentum equation}

Using (2.27) and (2.58) we get the relationship:

$$
\nabla_{0} \cdot\left(\sigma_{c}-p I\right)=0 .
$$

We now substitute (2.72) in (2.80) and get the momentum equation in the dofs and $c^{p g}$ :

$$
\nabla_{0} \cdot \sigma_{e}-c^{f} R T\left[\frac{\nabla_{0} \epsilon_{p 1}}{\epsilon_{\bar{p}}}+\Phi^{f} \frac{c^{p g} \nabla_{0} c^{p g}+\frac{1}{2}\left(c_{t}^{f}\right)^{2} \nabla_{0} \epsilon_{c}}{c^{\kappa}\left(c^{f}+c^{\kappa}\right)}\right]=\mathbf{0} .
$$

\subsubsection{The continuity equation}

We start out with (2.54), substitute (2.71) for $\rho^{ \pm}, \phi^{J} \rho_{t}^{J}$ for $\rho^{J}$, use (2.76) and (2.75), and get:

$$
-\boldsymbol{v}^{* f}=\phi^{f} \boldsymbol{K}^{*} \cdot\left[\xi \frac{\nabla_{0} \epsilon_{c}}{\epsilon_{c}}+\frac{\nabla_{0} \epsilon_{p}}{\epsilon_{p}}\right],
$$

with $\boldsymbol{K}^{*}$ a Lagrangian perneability tensor:

$$
\boldsymbol{K}^{*}:=c^{f} R T\left(\boldsymbol{B}^{s f}\right)^{-1}
$$

and:

$$
\xi:=\frac{1}{2}\left(M^{d i f} c^{p g}+M^{ \pm} c^{\kappa}\right) \frac{1}{\bar{M}^{ \pm} \bar{c}^{\prime}} .
$$

Multiplying the left and right hand sides of $(2.82)$ with $\phi^{J} \boldsymbol{F}$, taking the divergence with respect to the current configuration, and using the mass balance of the mixture (2.24), results into the continuity equation:

$$
\boldsymbol{\nabla} \cdot \boldsymbol{v}^{s}=\boldsymbol{\nabla} \cdot\left[\left(\phi^{\prime}\right)^{2} \boldsymbol{F} \cdot \boldsymbol{K}^{*} \cdot\left(\xi \frac{\boldsymbol{\nabla}_{0} \epsilon_{c}}{\epsilon_{c}}+\frac{\nabla_{0} \epsilon_{p}}{\epsilon_{p}}\right)\right] .
$$




\subsubsection{The diffusion equation}

We use the mass balance of the ions $(2.6)\left(\hat{c}^{ \pm}=0\right),(2.37)$, and (2.52) to arrive at an expression for the diffusion of the ions in terms of their density. The ion diffusion is given in terms of the gradient of the chemical potential of the ions and the Eulerian fluid velocity:

$$
\frac{\partial \rho^{ \pm}}{\partial t}=-\nabla \cdot\left[-\left(\rho^{ \pm}\right)^{2} \boldsymbol{F} \cdot\left(\boldsymbol{B}^{f \pm}\right)^{-1} \cdot \nabla_{0} \tilde{\mu}^{ \pm}+\rho^{ \pm} \boldsymbol{v}^{f}\right]
$$

Summing the terms $\frac{\partial \rho^{ \pm}}{\partial t}$ and $\nabla \cdot\left(\rho^{ \pm} v^{f}\right)$, and using the mass balance for the fluid frum (2.6) with $\hat{c}^{\jmath}=0$, and using (2.71), we get:

$$
\frac{\partial \rho^{ \pm}}{\partial t}+\nabla \cdot\left(\rho^{ \pm} v^{f}\right)=\phi^{f}\left[\gamma\left(c^{\dot{p} g}\right)_{f}+\beta\left(\dot{\epsilon}_{c}\right)_{f}\right]
$$

with:

$$
\begin{gathered}
\left(c^{\dot{p} g}\right)_{f}=\left(c^{\dot{p} g}\right)_{s}+\boldsymbol{v}^{-f} \cdot \nabla_{0} c^{p y} . \\
\left(\dot{\epsilon}_{c}\right)_{f}=\left(\dot{\epsilon}_{c}\right)_{s}+\boldsymbol{v}^{* f} \cdot \nabla_{0} \epsilon_{c}, \\
\gamma:=\frac{1}{2}\left(M^{d i f}+\frac{M^{ \pm} c^{p g}}{c^{k}}\right), \\
\beta:=\frac{M^{ \pm}\left(c_{ \pm}^{f}\right)^{2}}{4 c^{*}} .
\end{gathered}
$$

Substituting (2.87) in (2.86), using (2.75) yields the diffusion equation:

$$
\phi^{f}\left[\gamma\left(\dot{c}^{\dot{p} g}\right)_{f}+\beta\left(\dot{\epsilon}_{c}\right)_{f}\right]=\boldsymbol{\nabla} \cdot\left[\frac{\left(\rho^{ \pm}\right)^{2}}{\epsilon_{c}} \boldsymbol{F} \cdot \boldsymbol{D}^{*} \cdot \nabla_{\mathrm{u} \epsilon_{c_{c}}}\right],
$$

with:

$$
\boldsymbol{D}^{*}:=\frac{R T}{M^{ \pm}}\left(\boldsymbol{B}^{f \pm}\right)^{-1},
$$

the Lagrangian diffusion tensor.

2.4.5 Summary of differential equations and boundary conditions

The differential equations

1. The momentum equation:

$$
\boldsymbol{\nabla}_{0} \cdot \boldsymbol{\sigma}_{e}-c^{J} R T\left[\frac{\nabla_{0} \epsilon_{p}}{\epsilon_{p}}+\Phi^{f} \frac{c^{p g} \nabla_{0} c^{p g}+\frac{1}{2}\left(c_{ \pm}^{f}\right)^{2} \nabla_{0} \epsilon_{c}}{c^{\kappa}\left(c^{f}+c^{\kappa}\right)}\right]=0
$$

2. the continuity equation:

$$
\boldsymbol{\nabla} \cdot \boldsymbol{v}^{s}=\boldsymbol{\nabla} \cdot\left[\left(\phi^{\prime}\right)^{2} \boldsymbol{F} \cdot \boldsymbol{K}^{*} \cdot\left(\xi \frac{\boldsymbol{\nabla}_{0} \epsilon_{c}}{\epsilon_{c}}+\frac{\boldsymbol{\nabla}_{0} \epsilon_{p}}{\epsilon_{p}}\right)\right],
$$


3. the diffusion equation:

$$
\phi^{f}\left[\gamma\left(\dot{c}^{\dot{p} y}\right)_{f}+\beta\left(\dot{\epsilon}_{c}\right)_{f}\right]=\nabla \cdot\left[\frac{\left(\rho^{ \pm}\right)^{2}}{\epsilon_{c}} \boldsymbol{F} \cdot \boldsymbol{D}^{*} \cdot \nabla_{\alpha_{i}}\right],
$$

with:

$$
\begin{array}{r}
\sigma_{e}=(\operatorname{det} \boldsymbol{F})^{-1} \boldsymbol{F} \cdot \frac{\partial \psi^{j}}{\partial \boldsymbol{E}^{s}} \cdot \boldsymbol{F}^{c}, \\
\boldsymbol{K}^{*}=c^{f} R T\left(\boldsymbol{B}^{s f}\right)^{-1}, \\
\xi=\frac{1}{2}\left(M^{d i f} c^{p g}+M^{ \pm} c^{\kappa}\right) \frac{1}{M^{ \pm} c^{j}}, \\
\gamma=\frac{1}{2}\left(M^{d i f}+\frac{M^{ \pm} c^{p g}}{c^{\kappa}}\right), \\
\beta=\frac{M^{ \pm}\left(c_{ \pm}^{f}\right)^{2}}{4 c^{\kappa}}, \\
c_{ \pm}^{f}:=\frac{2 c^{f}}{\gamma^{ \pm}}, \\
c^{\kappa}:=\sqrt{\left(c^{p g}\right)^{2}+\left(c_{ \pm}^{f}\right)^{2} \epsilon_{i}}, \\
\rho^{ \pm}=\frac{1}{2} \phi^{f}\left[M^{d i f} c^{p g}+M^{ \pm} c^{\kappa}\right], \\
D^{*}=\frac{R I}{M^{ \pm}}\left(\boldsymbol{B}^{f \pm}\right)^{-1},
\end{array}
$$

The boundary conditions

All degrees of freedom have continuous boundary values. We use the superscript "out" for values just outside the boundary, and "in" for values just inside the boundary. We then get:

$$
\begin{gathered}
(\boldsymbol{u})^{\text {out }}=(\boldsymbol{u})^{\text {in }}, \\
\left(\epsilon_{p}\right)^{\text {out }}=\left(\epsilon_{p}\right)^{\text {in }}, \\
\left(\epsilon_{c}\right)^{\text {out }}=\left(\epsilon_{c}\right)^{\text {in }} .
\end{gathered}
$$




\section{Chapter 3}
A finite element formulation for the triphasic mechanics of cartilaginous materials based on the chemical potentials of fluid and ions 


\subsection{Introduction}

Different finite element models (FEM) (for textbooks see, e.g., Zienkiewicz ${ }^{238}$ and Bathe ${ }^{12}$ ) have been developed to solve the differential equations of bi- and triphasic models. Degrees of freedom for biphasic models can be: solid displacement and fluid pressure ( $\boldsymbol{u}$ $p$ ), or solid displacement and relative fluid displacement $(\boldsymbol{u}-\boldsymbol{w}) \cdot{ }^{198}$ In case fluid pressure is not a degree of freedom, an incompressibility constraint is accounted for by means of a penalty method. ${ }^{2016}$

Ion swelling was introduced in a FEM by Simon and Gaballa. ${ }^{195}$ They used an uncoupled differential equation for the ion diffusion, and imposed its effect upon the poro-elastic problem. The degrees of freedom were the solid displacement $\boldsymbol{u}$, a relative fluid displacement parameter and the local ion concentration. Snijders, ${ }^{200}$ et al. ${ }^{203}$ developed a FEM of the triphasic model with coupled differential equations. based on the degrees of freedom solid displacement $\boldsymbol{u}$, fluid pressure $p$, and ions density $\rho^{ \pm}$, of which the fluid pressure and ions density are not continuous at the boundaries. In this chapter a FEM is developed, based on the solid displacement, $\boldsymbol{u}$, and the chemical potentials of fluid and ions, which are continuous at boundaries. The differential equations derived in the previous chapter are written in finite element form, using the weighted residual method with a total Lagrange formulation. A one-dimensional element is developed, the results of which are compared with analytical solutions of a biphasic compression, and a diffusion problenn. Finally, experimental results of confined swelling and compression experiments are fitted using the $1 \mathrm{D}$ element. 


\subsection{The finite element model}

The differential equations, derived in the previous chapter, are written in a form that is suitable for the finite element (FE) solution process. They are written in an integral form using the weighted residual method. All equations are written with respect to a reference configuration (total Lagrange formulation).

For the weighted residual method, we multiply the differential equations with arbitrarily chosen weighting functions: $z$ for the momentum equation, $g$ for the continuity equation, and $\mathrm{h}$ for the diffusion equation. These equations are integrated over the current volume $V$. By applying Gauss' theorem, volume integrals are transformed to surface integrals. For the Lagrange formulation, the integrations are taken over the reference volume $V_{0}$, where it is understood that:

$$
\begin{array}{r}
d V=J d V_{0}, \\
\boldsymbol{n} d A=J \boldsymbol{F}^{-c} \cdot \boldsymbol{n}_{0} d A_{0},
\end{array}
$$

with:

$V=$ current volume,

$J=$ volume change factor,

$V_{0}=$ volume in the reference state,

$\boldsymbol{n}=$ surface outward normal in the current state,

$A=$ current surface,

$\boldsymbol{F}^{-c}=$ inverse of the conjugate of the deformation gradient tensor $\boldsymbol{F}$,

$\boldsymbol{n}_{0}=$ surface outward normal in the reference state,

$A_{0}=$ surface in the reference state.

For the transformation from current to reference configuration (see (2.39)), we introduce the effective second Piola Kirchhoff stress tensor $S$, defined by:

$$
\boldsymbol{S}=J \boldsymbol{F}^{-1} \cdot \boldsymbol{\sigma}_{e} \cdot \boldsymbol{F}^{-c}
$$

To transform the momentum equation we make use of some tensor/vector calculation rules:

$$
\begin{array}{r}
\boldsymbol{A}:(\boldsymbol{B} \cdot \boldsymbol{C})=(\boldsymbol{A} \cdot \boldsymbol{B}): \boldsymbol{C}, \\
\boldsymbol{A}^{c}: \boldsymbol{B}^{c}=\boldsymbol{A}: \boldsymbol{B}, \\
(\boldsymbol{A} \cdot \boldsymbol{a}) \cdot \boldsymbol{b}=\boldsymbol{A}: \boldsymbol{a b} .
\end{array}
$$

Applying the above to the differential equations (2.94)-(2.96), we get:

1. the momentum equation:

$$
\int_{V_{0}}\left(\nabla_{0} z\right)^{c}:\left[\boldsymbol{S} \cdot \boldsymbol{F}^{c}-p J \boldsymbol{F}^{-1}\right] d V_{0}=\int_{A_{0}} \boldsymbol{t} \cdot \boldsymbol{z} d A_{0},
$$

2. the continuity equation:

$$
\begin{aligned}
\int_{V_{0}}\left[g\left(\boldsymbol{F}^{-c} \cdot \nabla_{0}\right) \cdot \boldsymbol{v}^{s}+\left(\phi^{J}\right)^{2} \nabla_{0} g \cdot \boldsymbol{K}^{*} \cdot\left(\xi \frac{\boldsymbol{\nabla}_{0} \epsilon_{\varepsilon_{e}}}{\epsilon_{c}}\right.\right. & \left.\left.+\frac{\boldsymbol{\nabla}_{0} \epsilon_{p}}{\epsilon_{p}}\right)\right] J d V_{0} \\
& =\int_{A_{0}} g q^{J} d A_{0},
\end{aligned}
$$


3. the diffusion equation:

$$
\begin{array}{r}
\int_{V_{0}}\left[h \phi^{J}\left[\gamma\left(\dot{c}^{p g}\right)_{f}+\beta\left(\dot{\epsilon}_{c}\right)_{f}\right]+\frac{\left(\rho^{ \pm}\right)^{2}}{\epsilon_{c}} \nabla_{0} h \cdot D^{*} \cdot \nabla_{0} \epsilon_{i}\right] J d l_{0} \\
=\int_{A_{0}} h q^{i} d \Lambda_{0}
\end{array}
$$

with:

$\boldsymbol{t}=\left(\boldsymbol{F}^{-\boldsymbol{c}} \cdot \boldsymbol{n}_{0}\right) \cdot\left(\boldsymbol{\sigma}_{e}-p \boldsymbol{I}\right) J$,

$\xi=\frac{1}{2}\left[M^{d i f} c^{p g}+M^{ \pm} c^{\kappa}\right] \frac{1}{M^{ \pm c}}$,

$q^{f}=J\left(\phi^{f}\right)^{2} \boldsymbol{n}_{0} \cdot \boldsymbol{K}^{*} \cdot\left[\xi \frac{\boldsymbol{\nabla}_{0 \epsilon_{c}}}{\epsilon_{c}}+\frac{\nabla_{0 \epsilon_{P}}}{\epsilon_{p}}\right]$,

$\gamma=\frac{1}{2}\left(M^{d i \rho}+\frac{M^{ \pm} c^{p g}}{c^{x}}\right)$,

$\beta=\frac{M^{ \pm}\left(c_{x}^{\prime}\right)^{2}}{4 c^{x}}$,

$q^{i}=J \frac{\left(\rho^{ \pm}\right)^{2}}{\epsilon_{c}} n_{0} \cdot D^{*} \cdot \nabla_{0} \epsilon_{c}$.

In the solution process of the non-linear equations, the variables are estimated with a modified Newton-Raphson iterative procedure. The difference between these estimates and the correct value is indicated by a $\delta$ term. For instance, for $p$ we have: $p=\breve{p}+\delta p$. The estimate is marked by a " ". We express the estimates and deviations in dofs, and get, using linearizations:

$$
\begin{array}{r}
\breve{\boldsymbol{F}}=\boldsymbol{I}+\left(\boldsymbol{\nabla}_{0} \boldsymbol{u}^{*}\right)^{c}, \\
\delta \boldsymbol{F}=\left(\nabla_{0} \delta \boldsymbol{u}\right)^{c}, \\
\boldsymbol{F}^{-1}=\left(\boldsymbol{I}-\breve{\boldsymbol{F}}^{-1} \cdot \delta \boldsymbol{F}\right) \cdot \breve{\boldsymbol{F}}^{-1}, \\
J=\breve{J}\left(1+\breve{\boldsymbol{F}}^{-1}: \delta \boldsymbol{F}\right)=\breve{J}\left(1+\breve{\boldsymbol{F}}^{-1}:\left(\boldsymbol{\nabla}_{0} \delta \boldsymbol{u}\right)^{c}\right), \\
\delta \boldsymbol{S}=\frac{\partial \breve{\boldsymbol{S}}}{\partial \breve{\boldsymbol{E}}^{s}}: \delta \boldsymbol{E}^{s}, \\
\delta \boldsymbol{E}=\frac{1}{2}\left(\delta \boldsymbol{F}^{c} \cdot \breve{\boldsymbol{F}}+\breve{\boldsymbol{F}}^{c} \cdot \delta \boldsymbol{F}\right) .
\end{array}
$$

The quantities $c^{p g}$ and $\phi^{f}$ are both functions of the deformation. If we assume intrinsical incompressibility of the solid and fluid and negligible volume fraction of the ions (2.18), we can write $c^{p g}$ and $\phi^{f}$ in terms of their values in a reference state (indicated with subscript " 0 "), and the determinant $J$ of the deformation gradient tensor:

$$
\begin{gathered}
c^{p g}=\frac{c_{0}^{p g}}{1-\frac{1-J}{\phi_{0}^{\prime}}}, \\
\phi^{f}=1-\frac{1-\phi_{0}^{f}}{J} .
\end{gathered}
$$

The deviations of $c^{p g}$ and $\phi^{f}$ are written as a linear form in $\delta \boldsymbol{u}$, where we approximate $\breve{J}$ for $J$ :

$$
\delta c^{p g}=-\frac{c_{0}^{p g}}{\phi_{0}^{f}\left(1-\frac{1-J}{\phi_{0}^{J}}\right)^{2}} \breve{J}^{-1}:\left(\nabla_{0} \delta \boldsymbol{u}\right)^{c},
$$




$$
\delta \phi^{f}=\frac{1-\phi_{0}^{f}}{J} \breve{\boldsymbol{F}}^{-1}:\left(\boldsymbol{\nabla}_{0} \delta \boldsymbol{u}\right)^{c} .
$$

In the following, all deviations are expressed in those of the dofs $u, \epsilon_{p}$, and $\epsilon_{\varphi}$. In the processing of the equations we neglect the quadratic terms in the deviations. In stead of using the " " for the estimates, we will write them without this sign from now on. The" deviations of $p, \xi, \beta, \gamma$, are given in the appendix.

For the momentum equation we get:

$$
\begin{array}{r}
\int_{V_{0}}\left(\nabla_{0} \boldsymbol{z}\right)^{c}:\left[\boldsymbol{S} \cdot \boldsymbol{F}^{c}-p J \boldsymbol{F}^{-1}\right] d V_{0} \\
+\int_{V_{0}}\left(\nabla_{0} z\right)^{c}:\left[\left(\boldsymbol{F}^{c} \cdot \frac{\partial \boldsymbol{S}}{\partial \boldsymbol{E}^{s}} \cdot \boldsymbol{F}\right): \nabla_{0} \delta \boldsymbol{u}\right. \\
\left.+\boldsymbol{S} \cdot \boldsymbol{\nabla}_{0} \delta \boldsymbol{u}-\boldsymbol{F}^{-1}\left(\boldsymbol{F}^{-1}:\left(\boldsymbol{\nabla}_{0} \delta \boldsymbol{u}\right)^{c}\right) p_{1} J\right] d V_{0} \\
-\int_{V_{0}}\left(\nabla_{0} \boldsymbol{z}\right)^{c}: \boldsymbol{F}^{-1}\left(p_{2} \delta \epsilon_{p}+p_{3} \delta \epsilon_{c}\right) d V_{0} \\
=\int_{A_{0}}(\boldsymbol{t}+\delta \boldsymbol{t}) \cdot \boldsymbol{z} d A_{0} .
\end{array}
$$

with:

$$
\begin{array}{r}
p_{1}=-R T \Phi^{f} \frac{\left(c^{p g}\right)^{3}}{c^{\kappa}\left(1+\frac{c^{\kappa}}{c^{\jmath}}\right) \phi_{0}^{f} c_{0}^{p g}}, \\
p_{2}=R T \frac{c^{f}}{\epsilon_{p}}, \\
p_{3}=R T \Phi^{f} \frac{\left(c_{ \pm}^{f}\right)^{2}}{2 c^{\kappa}\left(1+\frac{c^{\kappa}}{c^{f}}\right)} .
\end{array}
$$

The continuity equation is approximated as:

$$
\begin{array}{r}
\int_{V_{0}}\left[g \nabla \cdot \boldsymbol{v}^{s}+\left(\phi^{f}\right)^{2}\left[\frac{\xi}{\epsilon_{c}} \nabla_{0} g \cdot \boldsymbol{K}^{*} \cdot \nabla_{0} \epsilon_{c}+\frac{1}{\epsilon_{p}} \nabla_{0} g \cdot \boldsymbol{K}^{*} \cdot \nabla_{0} \epsilon_{p}\right]\right] J d V_{0} \\
+\int_{V_{0}} g \nabla \cdot \delta \boldsymbol{v}^{s} J d V_{0} \\
\left.+\int_{V_{0}}\left(\phi^{\prime}\right)^{2}\left[\frac{\xi}{\epsilon_{c}} \nabla_{0} g \cdot \boldsymbol{K}^{*} \cdot \nabla_{0} \delta \epsilon_{c}+\frac{1}{\epsilon_{p}} \nabla_{0} g \cdot \boldsymbol{K}^{*} \cdot \nabla_{0} \delta \epsilon_{p}\right]\right] J d V_{0} \\
-\int_{A_{0}} g(q+\delta q) d A_{0}=0 .
\end{array}
$$

The diffusion equation is deduced, with the above linearizations and approximations:

$$
\begin{aligned}
& \int_{V_{0}}\left[h \phi^{f}\left(\gamma\left(\dot{c}^{p g}\right)_{j}+\beta\left(\dot{\epsilon}_{c}\right)_{j}+\frac{\left(\rho^{ \pm}\right)^{2}}{\epsilon_{\varepsilon}} \nabla_{0} h \cdot \boldsymbol{D}^{*} \cdot \nabla_{0} \epsilon_{c}\right] J d V_{0}\right. \\
+ & \int_{V_{0}}\left\{h \phi ^ { J } \left(\gamma \frac{c^{p g}}{\phi^{f}} \boldsymbol{F}^{-1}:\left(\nabla_{0} \delta \boldsymbol{v}^{s}\right)^{c}+\beta\left[\left(\delta \epsilon_{c}\right)_{s}+\boldsymbol{v}^{* J} \cdot \nabla_{0} \delta_{c_{c}}\right]\right.\right.
\end{aligned}
$$




$$
\begin{array}{r}
-\frac{\phi^{j} \xi}{\epsilon_{c}}\left[\gamma \nabla_{0} c^{p g}+\beta \nabla_{0} \epsilon_{c}\right] \cdot \boldsymbol{K}^{*} \cdot \nabla_{0} \delta \epsilon_{c} \\
\left.-\frac{\phi^{\prime}}{\epsilon_{p}}\left[\gamma \nabla_{0} c^{p g}+\beta \nabla_{0} \epsilon_{c}\right] \cdot \boldsymbol{K}^{*} \cdot \nabla_{0} \delta \epsilon_{p}\right) \\
\left.+\frac{\left(\rho^{ \pm}\right)^{2}}{\epsilon_{c}} \nabla_{0} h \cdot \boldsymbol{D}^{*} \cdot \nabla_{0} \delta \epsilon_{c}\right\} J d V_{0} \\
-\int_{A_{0}} h\left(q^{i}+\delta q^{i}\right) d \cdot \mathcal{A}_{0}=0 .
\end{array}
$$

\subsubsection{Discretization}

Spatial discretization To calculate the volume integrals from the previous section, the volume is divided into sub-volumes, the elements. The integral over the total volume is thus the sum of integrals over the elements. The elements consist of fixed sets of material points. Therefore, with deformation of the solid, the elements also change in shape. Within the elements, $n_{u}$ geometrical nodes are defined, with position vector $\boldsymbol{x}^{j}$ for the $\mathrm{j}$-th node $\left(\mathrm{j}=1, \ldots, n_{u}\right)$. For interpolation between the nodes, shape functions are defined. With the above definitions, the position vector $\boldsymbol{x}\left(\eta_{\alpha}\right)$ of a material point $\eta_{\alpha}$ is given by:

$$
\boldsymbol{x}\left(\eta_{\alpha}\right)=\varphi^{j}\left(\eta_{\alpha}\right) \boldsymbol{x}^{j} ; \quad j=1, \ldots, n_{u},
$$

with:

$\varphi^{j}=$ the shape function for the position vector belonging to node $j$,

$\eta_{\alpha}=$ the material coordinates $(\alpha=1,2,3)$,

$\boldsymbol{x}^{j}=$ the position vector of node $\mathrm{j}$,

$n_{u}=$ the number of geometrical nodes.

Note that we have used the summation convention over the indexes that appear twice. In the following we will use the summation convention unless otherwise indicated. The dofs, $\boldsymbol{u}, \epsilon_{p}, \epsilon_{c}$, are interpolated as follows:

$$
\begin{array}{rr}
\boldsymbol{u}=\varphi^{j} \boldsymbol{u}^{j}=\varphi^{j} u_{\alpha}^{j} \boldsymbol{g}_{\alpha i} \quad j=1, \ldots, n_{u ;} & \alpha=1,2,3, \\
\epsilon_{p}=\psi^{s} \epsilon_{j ;}^{s} ; & s=1, \ldots, n_{p}, \\
\epsilon_{c}=\chi^{w} \epsilon_{\varepsilon}^{w} ; & w=1, \ldots, n_{c},
\end{array}
$$

with:

$\varphi^{j}=$ the displacement shape function of node $\mathrm{j}$,

$\boldsymbol{u}^{j}=$ the displacement vector of node $\mathrm{j}$,

$\boldsymbol{g}_{\alpha}=$ a covariant base vector $(a=1,2,3)$,

$u_{\alpha}^{j}=\alpha$-th coordinate of displacement vector $\boldsymbol{u}^{j}$ of node $\mathrm{j}$ with respect to base vector $\boldsymbol{g}$,

$\psi^{s}=$ the $\epsilon_{p}$ shape function for node $s$,

$\chi^{w}=$ the $\epsilon_{c}$ shape function for node $w$,

$\hat{c}_{p}^{s}=$ the $\epsilon_{p}$ value in node $s$,

$\epsilon_{c}^{w}=$ the $\epsilon_{c}$ value in node $\mathrm{w}$. 
The weighting functions $\boldsymbol{z}, \mathrm{g}$, and h are interpolated in the same way as the dofs:

$$
\begin{gathered}
\boldsymbol{x}=\varphi^{i} z_{\alpha}^{i} g_{\alpha}, \\
g=\psi^{r} g^{r}, \\
h=\chi^{\nu} h^{\nu} .
\end{gathered}
$$

Time discretization We use a Houbolt ${ }^{12}$ third order time integration with variable time step. The Houbolt method yields for an arbitrary function $y(t)$ :

$$
\dot{y}(t)=A y(t)+B y\left(t-\tau_{1}\right)+C y\left(t-\tau_{2}\right)+D y\left(t-\tau_{3}\right)
$$

where $\tau_{i}$ are time constants, and $A, B, C$, and $D$ integration constants depending on the $\tau_{i}$. For the first two time steps a special starting procedure is needed. By definition (3.19) the deviation in the time derivative of the function $y$ is given by:

$$
\delta \dot{y}=A \delta y .
$$

Discretization of the weighted residual equations The volume integrals (3.12), (3.14), and (3.15) are calculated as the sum of integrals over the elements. Discretization of the element integrals leads to the following equations (momentum, continuity, diffusion):

$$
\begin{array}{r}
z_{\alpha}^{i}\left[\left(I_{\alpha \beta}^{i j}+(G E)_{\alpha \beta}^{i j}+P_{\alpha \beta}^{i j}\right) \delta u_{\beta}^{j}+R_{\alpha}^{i s} \delta \epsilon_{p}^{s}+O_{i \alpha}^{i w} \delta \epsilon_{c}^{w}=\left(F_{t x t}\right)_{\alpha}^{i}-\left(F_{i n t}\right)_{\alpha}^{i}\right], \\
g^{r}\left[A S_{\alpha}^{i r} \delta u_{\alpha}^{i}+L^{r \gamma} \delta \epsilon_{p}^{\gamma}+K^{r \nu} \delta \epsilon_{c}^{\nu}=Q_{e x t}^{r}-Q_{i n t}^{r}\right], \\
h^{\nu}\left[A T_{\alpha}^{i \nu} \delta u_{\alpha}^{i}+F^{\nu s} \delta \epsilon_{p}^{s}+\left[A M^{\nu w}+V^{\nu w}+D^{\nu w}+E^{\nu \nu w}\right] \delta \epsilon_{c}^{w}=C_{\varepsilon x t}^{\nu}-C_{i n t}^{\nu}\right] .
\end{array}
$$

The indexes $\alpha$ and $\beta$ run over the three covariant base vectors. The indexes $\mathrm{i}, \mathrm{r}, \nu, \mathrm{j}, \mathrm{s}, \mathrm{w}, \gamma$ run over the nodes $\left(n_{u}\right.$ geometrical nodes, $n_{p} \epsilon_{p}$ nodes and $n_{c} \epsilon_{c}$ nodes). The elements in the matrix (3.21) are:

momentum:

$$
\begin{aligned}
& I_{\alpha \beta}^{i j}=\int_{V_{0}^{e}}\left(\boldsymbol{\Phi}_{\alpha}^{i}\right)_{0}^{c}:\left(S \cdot\left(\boldsymbol{\Phi}_{\beta}^{j}\right)_{0}\right) d V_{0}^{e}, \\
& (G E)_{\alpha \beta}^{i j}=\int_{V_{0}^{c}}\left(\boldsymbol{\Phi}_{\alpha}^{i}\right)_{0}^{c}:\left[\left(\boldsymbol{F}^{c} \cdot \frac{\partial \boldsymbol{S}}{\partial \boldsymbol{E}^{s}} \cdot \boldsymbol{F}\right):\left(\boldsymbol{\Phi}_{\beta}^{j}\right)_{0}\right] d V_{0}^{e}, \\
& P_{\alpha \beta}^{i j}=-\int_{V_{0}^{e}} p_{1}\left(\boldsymbol{\Phi}_{\alpha}^{i}\right)_{0}^{c}: \boldsymbol{F}^{-1}\left(\boldsymbol{F}^{-1}:\left(\boldsymbol{\Phi}_{\beta}^{j}\right)_{0}^{c}\right) J d V_{0}^{e}, \\
& R_{* x}^{i s}=-\int_{V_{0}^{e}} p_{2} \psi^{s}\left(\Phi_{i x}^{i}\right)_{0}^{c}: \boldsymbol{F}^{-1} d V_{0}^{c} \\
& O_{\alpha}^{i w}=-\int_{V_{0}^{c}} p_{3} \chi^{\prime \prime}\left(\boldsymbol{\Phi}_{a}^{i}\right)_{0}^{c}: \boldsymbol{F}^{-1} d V_{0}^{e} \text {, } \\
& \left(F_{t x t}\right)_{i,}^{i}=\int_{A_{0}^{\epsilon}} \varphi^{i}(t+\delta t) \cdot g_{\alpha} d A_{0}^{\epsilon} \\
& \left(F_{\text {int }}\right)_{\alpha}^{i}=\int_{V_{u}^{r}}\left(\Phi_{, q}^{i}\right)_{0}^{c}:\left(\boldsymbol{S} \cdot \boldsymbol{F}^{c}-p J \boldsymbol{F}^{-1}\right) d V_{0}^{e},
\end{aligned}
$$


60

continuity:

$$
\begin{array}{r}
S_{\alpha}^{i r}=\int_{V_{0}^{e}} \psi^{r}\left(\boldsymbol{\Phi}_{\alpha}^{i}\right)_{0}^{c}: \boldsymbol{F}^{-1} J d V_{0}^{e}, \\
L^{r \gamma}=\int_{V_{0}^{e}}\left(\phi^{f}\right)^{2} \frac{J}{\epsilon_{p}} \psi_{0}^{r} \cdot \boldsymbol{K}^{*} \cdot \boldsymbol{\psi}_{0}^{\gamma} d V_{0}^{e}, \\
K^{r \nu}=\int_{V_{0}^{e}}\left(\phi^{f}\right)^{2} \frac{J \xi}{\epsilon_{c}} \psi_{0}^{r} \cdot \boldsymbol{K}^{*} \cdot \boldsymbol{\chi}_{0}^{\nu} d V_{0}^{e}, \\
Q_{e x t}^{r}=\int_{A_{0}^{e}} \psi^{r}(q+\delta q) d A_{0}^{e}, \\
Q_{\text {int }}^{r}=\int_{V_{0}^{-}}\left[\psi^{r}(\dot{J})_{s}+\left(\phi^{J}\right)^{2}\left(\frac{\xi}{\epsilon_{c}} \psi_{0}^{r} \cdot \boldsymbol{K}^{*} \cdot \nabla_{0} \epsilon_{c}\right.\right. \\
\left.\left.+\frac{1}{\epsilon_{p}} \boldsymbol{\psi}_{0}^{r} \cdot \boldsymbol{K}^{*} \cdot \nabla_{0} \epsilon_{p}\right) J\right] d V_{0}^{\prime e},
\end{array}
$$

diffusion:

$$
\begin{aligned}
& T_{\alpha}^{i \nu}=\int_{V_{0}^{e}} \chi^{\nu} \gamma c^{p g} \boldsymbol{F}^{-1}:\left(\boldsymbol{\Phi}_{\alpha}^{i}\right)_{0}^{\varepsilon} J d V_{0}^{e}, \\
& F^{\nu s}=\int_{V_{0}^{e}} \chi^{\nu} \frac{\left(\phi^{J}\right)^{2}}{\epsilon_{p}}\left[\gamma \nabla_{0} c^{p g}+\beta \nabla_{0} \epsilon_{c}\right] \cdot \boldsymbol{K}^{*} \cdot \psi_{0}^{s} J d V_{0}^{e}, \\
& M^{\nu w}=\int_{V_{0}^{e}} \chi^{\nu} \chi^{w} \phi^{f} \beta J d V_{0}^{e}, \\
& V^{\nu w}=\int_{V_{0}^{e}} \chi^{\nu} \phi^{f} \beta v^{* f} \cdot \chi_{0}^{w} J d V_{0}^{e}, \\
& D^{\nu w}=\int_{V_{0}^{e}} \frac{\left(\rho^{ \pm}\right)^{2}}{\epsilon_{c}} \boldsymbol{\chi}_{0}^{\nu} \cdot \boldsymbol{D}^{*} \cdot \boldsymbol{\chi}_{0}^{w} J d V_{0}^{e}, \\
& E^{\nu w}=-\int_{V_{G}^{e}} \chi^{\nu}\left(\phi^{J}\right)^{2} \frac{\xi}{\epsilon_{c}}\left[\gamma \nabla_{0} c^{p g}+\beta \nabla_{0} \epsilon_{c}\right] \cdot K^{*} \cdot \chi_{0}^{w} J d V_{0}^{e}, \\
& C_{e x q}^{\nu}=\int_{A_{0}^{e}} \chi^{\nu}\left(q^{i}+\delta q^{i}\right) d A_{0}^{e}, \\
& C_{\text {int }}^{\nu}=\int_{V_{0}^{e}}\left(\chi^{\nu}\left[-\gamma c^{p g}\left\{(j)_{s}+\boldsymbol{v}^{* f} \cdot \boldsymbol{F}^{-\varepsilon} \cdot \nabla_{0} J\right)\right\}\right. \\
& \left.+\phi^{f} \beta\left(\left(\dot{\epsilon}_{c}\right)_{s}+\boldsymbol{v}^{* f} \cdot \boldsymbol{F}^{-c} \cdot \nabla_{0} \epsilon_{c}\right) J\right] \\
& \left.+\frac{\left(\rho^{ \pm}\right)^{2}}{\epsilon_{c}} \chi_{0}^{\nu} \cdot \boldsymbol{D}^{*} \cdot \nabla_{0} \epsilon_{c} J\right) d V_{0}^{e},
\end{aligned}
$$

with definitions:

$$
\begin{array}{r}
\left(\boldsymbol{\Phi}_{\alpha}^{i}\right)_{0}=\nabla_{0}\left(\varphi^{i} \boldsymbol{g}_{\alpha}\right), \\
\psi_{0}^{r}=\nabla_{0} \psi^{r}, \\
\chi_{0}^{\nu}=\nabla_{0} \chi^{\nu} .
\end{array}
$$




\subsection{The 1D computer implementation}

A one-dimensional element was implemented in a computer program for the simulation of confined swelling and compression experiments. We used linear shape functions, and constructed a symmetrical stiffness matrix. The reference height of the elements is $h_{0}$, the actual height is $h$. The elements have 2 nodes each. The isoparametric coordinate $\eta$ runs from -1 to +1 over an element. We define node 1 , referred to with sub- or superscript "1", at $\eta=-1$ and node 2, referred to with sub- or superscript "2", at $\eta=1$. The shape functions $\varphi^{i}(\eta), \chi^{i}(\eta), \psi^{i}(\eta)$ for the displacement $u, \epsilon_{p}$, and $\epsilon_{c}$ are defined as follows:

$$
\varphi^{i}(\eta)=\chi^{i}(\eta)=\psi^{i}(\eta):=\frac{1}{2}\left(1+\eta(-1)^{i}\right)
$$

where $i=1$ for node 1 , and $i=2$ for node 2 . The value for $\epsilon_{p}(\eta)$ at material point $\eta$ is thus given by:

$$
\epsilon_{p}(\eta)=\frac{1}{2} \epsilon_{p 1}(1-\eta)+\frac{1}{2} \epsilon_{p 2}(1+\eta),
$$

for $\eta$ from -1 to $+1 . \epsilon_{p 1}$ is the value of $\epsilon_{p}$ in node $1, \epsilon_{p 2}$ is the value of $\epsilon_{p}$ in node 2 . For the one-dimensional situation, we have the following definitions:

$$
F=\frac{h}{h_{0}} ; \quad J=\frac{h}{h_{0}} ; \quad S=\frac{h_{0}}{h} \sigma_{e}=\frac{1}{J} \sigma_{e},
$$

with:

$F=$ the deformation gradient,

$J=$ the volume (length) quotient,

$S=$ the effective second Piola-Kirchboff stress,

$\sigma_{e}=$ the effective Cauchy stress.

The element stiffness matrix ELSTIF is given by:

$\left[\begin{array}{cccccc}(I(G E) P)^{11} & R^{11} & O^{11} & (I(G E) P)^{12} & R^{12} & O^{12} \\ A S^{11} & L^{11} & K^{11} & A S^{21} & L^{12} & K^{12} \\ A T^{11} & F^{11} & ((A M) V D E)^{11} & A T^{21} & F^{12} & ((A M) V D E)^{12} \\ (I(G E) P)^{21} & R^{21} & O^{21} & (I(G E) P)^{22} & R^{22} & O^{22} \\ A S^{12} & L^{21} & K^{21} & A S^{22} & L^{22} & K^{22} \\ A T^{12} & F^{21} & ((A M) V D E)^{21} & A T^{22} & F^{22} & ((A M) V D E)^{22}\end{array}\right]$

with:

$(I(G E) P)^{i j}=I^{i j}+(G E)^{i j}+P^{i j}, i=1,2 ; j=1,2$,

$((A M) V D E)^{i j}=A M^{i j}+V^{i j}+D^{i j}+E^{i j}, i=1,2 ; j=1,2$,

$A=$ time integration constant.

The set of equations to be solved iteratively such that the deviations $\delta u_{i}, \delta \epsilon_{p i}$, and $\delta \epsilon_{c i}$, 
$i=1,2$, tend to zero is:

$$
[\operatorname{ELSTIF}]\left[\begin{array}{c}
\delta u_{1} \\
\delta t_{p_{1}} \\
\delta \epsilon_{11} \\
\delta u_{2} \\
\delta e_{p 2} \\
\delta \epsilon_{c 2}
\end{array}\right]=\left[\begin{array}{c}
F_{\text {ext }}^{1}-F_{i n t}^{1} \\
Q_{e r t}^{1}-Q_{i n t}^{1} \\
C_{e r t}^{1}-C_{i n t}^{1} \\
F_{\text {ext }}^{2}-F_{i n t}^{2} \\
Q_{t x t}^{2}-Q_{i n t}^{2} \\
C_{e x t}^{2}-C_{i n t}^{2}
\end{array}\right]
$$

A symmetrical nuatrix was devised. For this we approximated $R^{i j} . R^{i j}$ is given by:

$$
R^{i j}=-\frac{1}{4} c^{J} R T \frac{h}{h_{0}}(-1)^{i} \int_{-1}^{1} \frac{1+\eta(-1)^{j}}{\frac{1}{2} \epsilon_{p 1}(1-\eta)+\frac{1}{2} \epsilon_{p 2}(1+\eta)} d \eta
$$

$S^{i j}$ is given by:

$$
S^{i j}=\frac{1}{4}(-1)^{i} \int_{-1}^{1}\left(1+\eta(-1)^{j}\right) d \eta
$$

We approximated for $R^{i j}$ : a

$$
R^{i j} \approx-\frac{1}{4} c^{f} R T(-1)^{i} \int_{-1}^{1}\left(1+\eta(-1)^{j}\right) d \eta=-c^{J} R T S^{i j}
$$

The above approximation is valid for values of $\epsilon_{p 1}$ and $\epsilon_{p 2}$ close to unity, and $h$ close to $h_{0}$. Using approximation (3.33), we write $R^{i j}=-c^{f} R T S^{i j}$, and multiply the second and fifth rows with $\frac{c^{f} R T}{-A}$. If we further leave out the remaining non-symmetrical terms, we get:

$$
\left[\begin{array}{cccccc}
(I(G E) P)^{11} & -c^{f} R T S^{11} & 0 & (I(G E) P)^{12} & -c^{f} R T S^{12} & 0 \\
-c^{f} R T S^{11} & \left(\frac{c^{f} R T}{-A}\right) L^{11} & 0 & -c^{f} R T S^{21} & \left(\frac{c^{f} R T}{-A}\right) L^{12} & 0 \\
0 & 0 & ((A M) D)^{11} & 0 & 0 & ((A M) D)^{12} \\
(I(G E) P)^{12} & -c^{J} R T S^{21} & 0 & (I(G E) P)^{22} & -c^{f} R T S^{22} & 0 \\
-c^{J} R T S^{12} & \left(\frac{c^{f} R T}{-A^{-A}}\right) L^{12} & 0 & -c^{f} R T S^{12} & \left(\frac{c^{f} R T}{-A}\right) L^{22} & 0 \\
0 & 0 & ((A M) D)^{21} & 0 & 0 & ((A M) D)^{22}
\end{array}\right]
$$

with:

$((A M) D)^{i j}=A M^{i j}+D^{i j} ; i=1,2 ; j=1,2$.

The corresponding right hand side is:

$$
\left[\begin{array}{c}
F_{\text {ext }}^{1}-F_{\text {int }}^{1} \\
\frac{c^{\prime} F T}{-A}\left(Q_{\text {ext }}^{1}-Q_{\text {int }}^{1}\right) \\
C_{\text {ext }}^{1}-C_{\text {int }}^{1} \\
F_{\text {ext }}^{2}-F_{\text {int }}^{2} \\
\frac{c^{t} R T}{-A}\left(Q_{\text {ext }}^{2}-Q_{\text {int }}^{2}\right) \\
C_{\text {ext }}^{2}-C_{\text {int }}^{2}
\end{array}\right]
$$

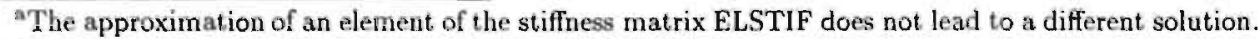
It only changes the direction in which a solution is sought. Ultimately, when the set of equations (3.30) is solved (within a preset accuracy), the magnitude of the elements in ELSTIF is of no importance. 
The expressions for the remaining elements in the symmetrical stiffness matrix are:

$$
\begin{aligned}
& I^{i j}=\frac{\sigma_{\varepsilon}}{h}(-1)^{i+j}, \\
& G E^{i j}=\frac{h^{2}}{h_{0}^{3}} \frac{\partial S}{\partial E^{s}}(-1)^{i+j}, \\
& P^{i j}=\frac{h_{0}}{2 h^{2}} \frac{R T \Phi^{J}}{n_{0}^{J} c_{0}^{p g}}\left(c^{p g}\right)^{3}(-1)^{i+j} \int_{-1}^{1} \frac{d \eta}{\left(1+\frac{1}{d} c^{\kappa}\right) c^{\kappa}}, \\
& L^{i j}=\frac{1}{2} \frac{h}{h_{0}^{2}}\left(\phi^{J}\right)^{2} K(-1)^{i+j} \int_{-1}^{1} \frac{d \eta}{l_{p}}, \\
& M^{i j}=\frac{h M^{ \pm}\left(c^{j}\right)^{2} \phi^{J}}{8\left(\gamma^{ \pm}\right)^{2}} \int_{-1}^{1} \frac{\left(1+\eta(-1)^{i}\right)\left(1-\eta(-1)^{j}\right)}{c^{\kappa}} d \eta, \\
& D^{i j}=\frac{R T\left(\phi^{f}\right)^{2}}{8 h_{0} M^{ \pm}}(-1)^{i+j} D \int_{-1}^{1} \frac{1}{\epsilon_{c}}\left[\left(\left(M^{ \pm}\right)^{2}+\left(M^{\alpha i j}\right)^{2}\right)\left(c^{p s}\right)^{2}+\right. \\
& \left.\left(M^{ \pm}\right)^{2}\left(c_{ \pm}^{J}\right)^{2} \epsilon_{c}+2 M^{ \pm} M^{r d i} c^{p g} c^{\kappa}\right] d \eta .
\end{aligned}
$$

The one-dimensional forms of the internal forces are:

$$
\begin{aligned}
& \left(F_{\text {int }}\right)^{i}=(-1)^{i}\left(\sigma_{e}-\frac{1}{2} R T c^{J} \int_{-1}^{1} \ln \left[\frac{t_{p}}{\gamma^{\prime}}\left(1+\frac{\tilde{c}^{\alpha}}{c^{f}}\right] d \eta\right),\right. \\
& \left(Q_{\text {int }}\right)^{i}=\frac{v_{2}^{s}-v_{1}^{s}}{2}+\frac{h}{h_{0}^{2}} K\left(\phi^{f}\right)^{2}(-1)^{i}\left(\frac{\epsilon_{c 2}-\epsilon_{c 1}}{4 M^{ \pm} c^{f}} \int_{-1}^{1} \frac{M^{d i f} c^{p g}+M^{ \pm} c^{\kappa}}{\epsilon_{c}} d \eta+\ln \frac{\epsilon_{p 2}}{\epsilon_{p 1}}\right) \text {, } \\
& \left(C_{\text {int }}\right)^{i}=\frac{h}{4} \int_{-1}^{1}\left(1+\eta(-1)^{i}\right)\left(\frac{1}{2}\left[M^{d i f}+\frac{M^{ \pm} c^{p g}}{c^{\alpha}}\right]\left[-c^{p g} \frac{v_{2}^{p}-v_{1}^{f}}{h}+\phi^{f}\left(v^{f}-v^{s}\right) \frac{\frac{\rho}{p g}^{p}-c^{p g}}{h}\right]\right. \\
& \left.+\phi^{f} \frac{M^{ \pm}\left(c_{ \pm}^{f}\right)^{2}}{4 c^{\kappa}}\left[\frac{1}{2}(1-\eta)\left(\dot{\epsilon}_{c 1}\right)_{s}+\frac{1}{2}(1-\eta)\left(\dot{\epsilon}_{\mathrm{c} 2}\right)_{s}+\left(v^{f}-v^{s}\right) \frac{\epsilon_{c}-\epsilon_{c 1}}{h}\right]\right) d \eta \\
& +\frac{h}{8 h_{0}^{2}}\left(\phi^{J}\right)^{2} D\left(\epsilon_{c 2}-\epsilon_{c 1}\right)(-1)^{i} \int_{-1}^{1} \frac{\left(M^{d i f} c^{p D}+M^{ \pm} \epsilon^{\kappa}\right)^{2}}{\epsilon_{c}} d \eta
\end{aligned}
$$

\subsubsection{Deformation dependencies; constitutive behaviour}

A number of model quantities are deformation dependent. We have already mentioned the fixed charge density and the porosity (3.8) and (3.9). The permeability and the diffusion coefficients $K^{*}$ and $D^{*}$ are also deformation dependent. The principle of equipresence would mean that $K^{*}$ and $D^{*}$ are dependent on all of the independent fields. In the literature, data are given on the dependency of $K^{*}$ and $D^{*}$ on fluid content. We will limit ourselves to the dependencies of $K^{*}$ and $D^{*}$ on fluid content, which is a function of the deformation.

The definitions of $K^{*}$ and $D^{*}$ we use in this numerical formulation differ from those normally encountered in the literature. To get values that can be compared with literature values (permeability coefficient in $\mathrm{m}^{4} / \mathrm{Ns}$, diffusion coefficient in $\mathrm{m}^{2} / \mathrm{s}$ ) we convert our $K^{*}$ and $D^{*}$ values:

$$
\begin{gathered}
K=K^{*} \frac{h^{2}}{\left(h_{0}\right)^{2}} ; \quad K_{t r i}=K \frac{\phi^{2}}{c^{J} R T}, \\
D=D^{*} \frac{h^{2}}{\left(h_{0}\right)^{2}} ; \quad D_{t r i}=D \frac{\left(\rho^{ \pm}\right)^{2}}{M^{ \pm}}\left(\frac{1}{c^{-}}+\frac{1}{c^{+}}\right) .
\end{gathered}
$$


The Lagrangian forms $K^{*}$ and $D^{*}$ are converted to Eulerian forms, $K$ and $D$, by multiplying with the factor $\frac{h^{2}}{\left(h_{0}\right)^{2}} . h_{0}$ is the height in the reference state of the sample. We shall use the stress free (subscript "sf") state of the sample as the reference state. The triphasic Eulerian permeability and diffusion coefficients (converted according to (3.38) and (3.39)) are referred to as " $K_{t r i}$ ", and " $D_{t r i}$ ", respectively. $K_{t r i}$ and $D_{t r i}$ are defined in the usual units of $\mathrm{m}^{4} / \mathrm{Ns}$, and $\mathrm{m}^{2} / \mathrm{s}$, respectively. To discern between permeability coefficients determined using biphasic theory and triphasic theory, biphasic permeability coefficients are referred to with the symbol " $K_{b i}$ " ( $K_{b i}$ is also defined in units of $\mathrm{m}^{1} / \mathrm{Ns}$ ). Note that $K$ and $D$ are deformation dependent, when $K_{t r i}$ and $D_{\text {tri }}$ respectively are assumed constant, because of the deformation dependencies of $\phi, \rho^{ \pm}$, and $c^{+}$and $c^{-}$.

The permeability is a function of the hydration. It increases as the hydration increases. We determined a $K_{b i}$ as a function of the hydration by fitting the compression phase of CSC experiments with a biphasic solution (chapters 4 and 5 ). The $K_{6 i}$-hydration data was fitted with a quadratic function:

$$
h_{h i}=0 . \bar{i} 10 *(h y d)^{2}-0.482 * h y d,
$$

where $K_{6 i}$ is in units of $10^{-16} \mathrm{~m}^{4} / \mathrm{Ns}$, and hyd is the hydration in $\mathrm{g} \mathrm{H}_{2} \mathrm{O}$ per g dry weight. The $K_{b i}$-hydration relationship (3.40) is similar to a relationship found by Urban and Maroudas. ${ }^{222}$ We used the $K_{b i}$-hydration relationship in our model as an estimate for the $K_{t r}$-hydration relationship, with addition of the constant $\Delta K_{t r i}$. We thus get as estimate for $K_{\text {tri }}$ :

$$
K_{\text {tri }}=0.710 *(h y d)^{2}-0.482 * h y d+\Delta K_{\text {tri }} .
$$

The diffusion of ions in a porous medium depends on the charges (fixed or mobile) present in the medium, and the concentration gradient of the mobile ions. The volume fractions of fluid and solid also determine the diffusional properties of the mobile ions in the material, because solid particles obstruct the movement of the ions. A formula, derived by Mackie and Meares ${ }^{119}$ for porous media, and based on purely geometrical considerations relates the diffusion coefficient in a porous medium, $D^{l}$, to that in free solution $D_{\text {free }}^{l}$. We use this formula to define $D_{\text {tri }}$ :

$$
D_{t r i}:=D^{l}=D_{f r e e}^{l} \frac{\left(\phi^{f}\right)^{2}}{\left(2-\phi^{f}\right)^{2}}
$$

In the model, diffusion of positive and negative ions is coupled into diffusion of the neutral salt. For the diffusion coefficient of the salt, the harmonic average of the diffusion coefficients of the separate ions has been used. Thus, for $\mathrm{NaCl}$, the diffusion coefficient in free solution is defined by:

$$
D_{\text {free }}^{\mathrm{NaCl}}=\frac{2}{\frac{1}{D^{\mathrm{Na}} \mathrm{t}}+\frac{1}{D^{C l^{-}}}},
$$

where $D^{N a^{+}}$and $D^{C^{-}}$are the diffusion coefficients of $\mathrm{Na}^{+}, \mathrm{Cl}^{-}$respectively, in an aqueous solution at physiological strength $(0.15 \mathrm{M} \mathrm{NaCl}),(3.43)$ is used to calculate the $D_{\text {free }}$ in (3.42). At $25^{\circ} \mathrm{C}$ the values for the diffusion coefficients are: ${ }^{129} D^{N \alpha^{+}}=1.33^{*} 10^{-9} \mathrm{~m}^{2} / \mathrm{s}$; $D^{C l^{-}}=2.04^{*} 10^{-9} \mathrm{~m}^{2} / \mathrm{s} ; D_{\text {free }}^{\mathrm{NaCl}}=1.61^{*} 10^{-9} \mathrm{~m}^{2} / \mathrm{s}$. 
The average activity coefficient of the ions $\gamma^{ \pm b}$ depends on the fixed charge density and the concentrations of the ions. When its value is not measured, it can be estimated from semi-empirical equations (Manning, ${ }^{121} \mathrm{Kwak}^{106}$ and Wells ${ }^{233}$ ). According to these relationships, the average activity coefficient is the product of a mobile-mobile ion interaction part, $\gamma^{M M}$, and a poly-ion/mobile ion interaction part, $\gamma^{P M}$. For the mobile-mobile ion interaction the relationship between $\gamma^{M M}$ and the ion concentration at the co-ion (i.e. the anion) concentration was used. For the calculation of $\gamma^{M M}$, data relating the average activity coefficient, $\gamma^{ \pm}$, to $\mathrm{NaCl}$ concentration in aqueous solutions, reproduced in Maroudas, ${ }^{128}$ was used. For the poly ion-mobile ion interaction, $\gamma^{P M}$, the equations proposed by Manning ${ }^{121}$ (equations also in Urban et al. $^{223}$ ) were used, using the average of the concentrations of the cations and anions. In chapter 5 the procedure for determining the average ionic activity coefficients in equilibrium situations in CSC experiments is given.

For the effective stress-strain relationship a linear relationship between the effective 2nd Piola-Kirchhoff stress $S$ and the Green-Lagrange strain $E^{3}$ of the solid was chosen:

$$
S=H_{e} * E^{s}
$$

where $H_{e}$ is the effective linear stiffness. The Green-Lagrange strain $E^{s}$ is defined relative to the a-priori unknown stress-free height.

\subsection{Comparison of the $1 \mathrm{D}$ model with analytical so- lutions}

The 1D computer model was tested against analytical solutions. As there are no analytical solutions for the differential equations of the model, solutions of a linear biphasic confined compression and a diffusion problem were used for comparisons. For the biphasic problem, ions diffusion, and osmotic pressure were switched off. For the diffusion problem, solid displacement, and fluid flow were switched off.

\subsubsection{Linear biphasic compression}

The linear biphasic confined compression test consists of compressing a sanople, confined in a cylindrical chamber with a force $F$. The bottom of the chamber is permeable for fluid. The top of the chamber consists of an impervious piston. The experimental setup is that of the confined swelling and compression experiment, described in section 1.3.3, Figure 1.7. The sample consists of a porous linearly elastic solid, the pores of which are filled with a fluid, normally water. Both the solid and the fluid are intrinsically incompressible. The sample has height $h$, cross section $A$, a compressive aggregate modulus (stiffness) $H_{A}$, and a permeability coefficient $K_{b i} . H_{A}$ and $K_{b i}$ are assumed to be constant (independent of deformation). The fluid pressure $p$ during linear biphasic compression is determined

\footnotetext{
${ }^{b}$ In the numerical formulation $\gamma^{ \pm}$is defined on the basis of the total fluid volume in the mixture. In chapter 5 average ions activity coefficients $\gamma_{e x \rho}^{ \pm}$, and $\gamma_{10 t}^{ \pm}$are defined, respectively on extrafibrillar and total fluid volume basis.
} 
from the following differential equation: ${ }^{164,211}$

$$
\frac{\partial p}{\partial t}=H_{A} K_{b i} \frac{\partial^{2} p}{\partial x^{2}}
$$

where $t=$ time, and $x=$ distance in the direction of the compressive force.

The differential equation (3.45) can be written with non-dimensional variables (Figure $3.2)$ :

$$
X:=\frac{x}{h} ; \quad T:=\frac{t}{\tau_{b i}}=\frac{H_{A} K_{b i}}{h^{2}} t ; \quad P:=\frac{A}{F} p,
$$

where $\tau_{b i}=$ compression time constant. Substitution of (3.46) in (3.45) yields:

$$
\frac{\partial P}{\partial T}=\frac{\partial^{2} P}{\partial X^{2}} .
$$

The initial condition of the experiment is given by:

$$
P=1, \text { for } 0 \leq X \leq 1 \text { at } T=0 .
$$

The boundary conditions are given by:

$$
\begin{aligned}
P & =0 \quad \text { at } X=0 \quad \text { (free drainage), } \\
\frac{\partial P}{\partial X}=0 \quad \text { at } X & =1 \quad \text { (no flow through } X=1) .
\end{aligned}
$$

The analytical solution of this problem is given by: ${ }^{35}$

$$
P=\sum_{n=0}^{\infty} \frac{2}{M} \sin (M X) e^{-M^{2} T},
$$

where $M=\frac{p i}{2}(2 n+1)$.

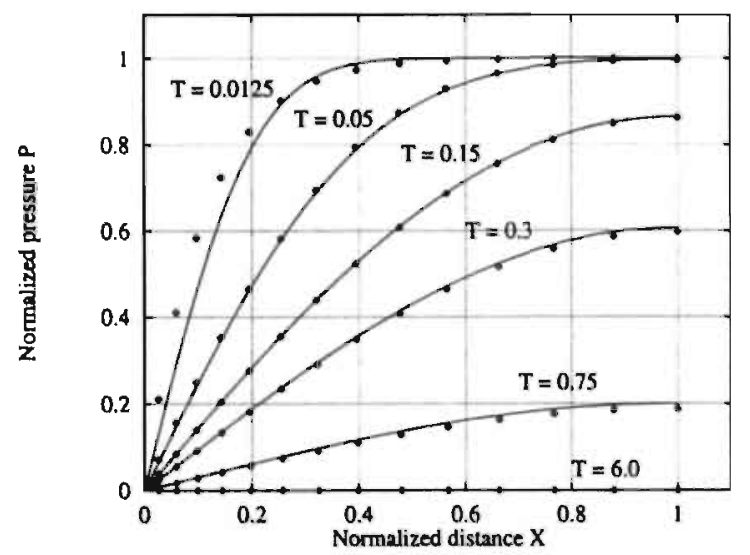

In order to simulate a biphasic linear compression with the triphasic computer model, the osmotic pressure was shut off by setting the $c^{p s}$, and $c^{+}$and $c^{-}$values to zero. In this case, the compressive force is only counteracted by the elastic (solid deformation) and viscous (fluid flow) forces. In Figure 3.1 the comparison between the analytical, and numerical results for $\tau_{b i}=400 \mathrm{~s}$ is shown. We see that the agreement between the analytical and numerical soFigure 3.1: Comparison between analytical (solid lines), and lutions is good. For $T=0.0125$ a numerical (diamonds) solutions of a linear biphasic confined small difference between the two compression experiment. The time constant $\tau_{b i}$ equals $400 \mathrm{~s}$, solutions is found. $H_{A}=5 \mathrm{MPa}, K_{b i}=5^{*} 10^{-16} \mathrm{~m}^{4} / \mathrm{Ns}_{1} h=1 \mathrm{~mm}$. 


\subsubsection{Diffusion}

Consider the situation of two solutions of $\mathrm{NaCl}$ in water, I and II, brought in contact with each other at time $t=0$. Solution $\mathrm{I}$ is of lower strength (concentration

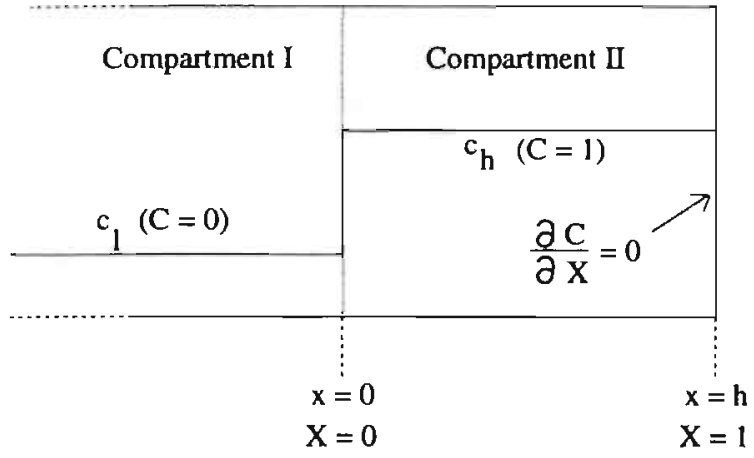

Figure 3.2: Schematic representation of one-dimensional diffusion between two compartments. The concentration at $\mathrm{t}=0$ $s$ is $c_{l}(C=0)$ in compartment $I$, and $c_{h}(C=1)$ in compartmeat II. Compartment II has length $h$. $c_{l}$ ) compared to solution II (con* centration $c_{h}$ ). Compartment. II has length $h$, compartment II is of infinite length (Figure 3.2). Because of the concentration difference, solute (salt molecules) from compartment II diffuses into compartment $I$. The rate of diffusion is determined by the concentration gradient, and the diffusion coefficient, $D^{l}$. Assuming a constant $D^{\prime}$, the differential equation that describes the concentration $c$ as a function of time and place in compartment II during the diffusion process is given by:

$$
\frac{\partial c}{\partial t}=D^{l} \frac{\partial^{2} c}{\partial x^{2}}
$$

Introducing dimensionless quantities:

$$
X:=\frac{x}{h} ; \quad T:=\frac{t}{\tau_{d}}=t \frac{D^{l}}{h^{2}} ; \quad C:=\frac{c-c_{l}}{c_{h}-c_{l}}
$$

where $\tau_{d}=$ diffusion time constant, we get substituting (3.52) into (3.51):

$$
\frac{\partial C}{\partial T}=\frac{\partial^{2} C}{\partial X^{2}}
$$

which is identical to (3.47), when $C$ is substituted by $P$. The diffusion problem also has the same initial (3.48), and boundary conditions (3.49) as the biphasic problem (with $C$ substituted for $P$ ), and thus the same solution (3.50). The initial condition $C=1$ for $0 \leq \mathrm{X} \leq 1$ at $T=0$ means that the initial concentration inside compartment II equals $c_{h}$. In compartment $I$, the concentration for $T \geq 0$ equals $c_{l}$ (first boundary condition). At $X=1$, compartment II is impermeable (no solute flow at $X=1$ boundary; second boundary condition).

The simulation of the above described diffusion process was performed by the computer program. The nodal displacements were set to zero, as was $c^{p g}$. By set- 


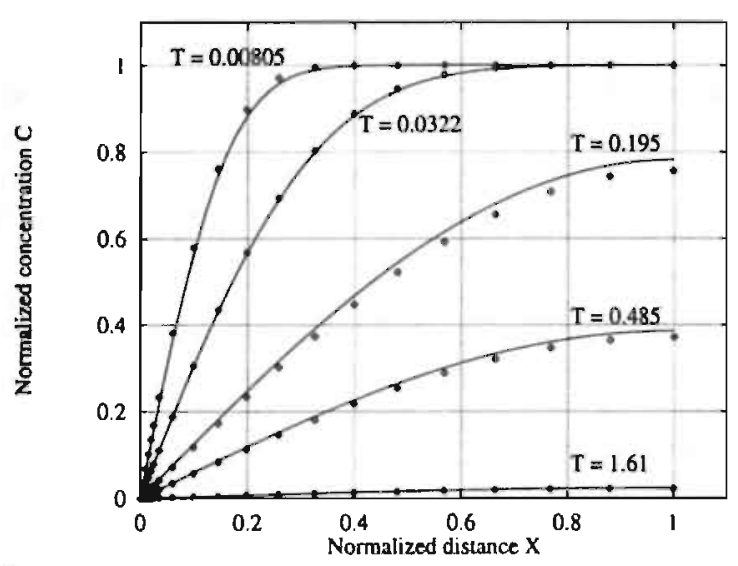

Figure 3.3: Comparison between analytical (solid lines), and numerical (diamonds) solutions of a diffusion problem. The time constant $\tau_{d}$ equals $621 \mathrm{~s}, h=1 \mathrm{~mm}$.

efficient for $\mathrm{NaCl}$ in water at $25^{\circ} \mathrm{C}$ ). We see that the numerical and analytical models show small differences for $\mathrm{T}=0.195$ and $\mathrm{T}=0.485$. These differences are possibly due to the approximation of $D$ from $D_{t r i}$ in eq. (3.39).

\subsection{The $1 \mathrm{D}$ computer simulation of CSC experiments}

In a confined swelling and compression experiment (CSC) a cylindrically shaped tissue sample is tightly fitted in a cylindrical chamber. The top of the chamber is a piston via which a mechanical load can be applied. The bottom of the chamber is closed by a filter through which a salt solution circulates (Figure 1.7a). Water can be expelled out of the sample through the filter by means of a step increase of the force on the piston (mechanical load) or by means of a step increase of the salt concentration in the filter (chemical load). The height of the sample is a function of the mechanical and chemical loads, and time. In a CSC, the sample height is measured as a function of time.

In this section we outline the simulation of the 1D program of two CSC experiments performed in our laboratories. The samples were specimens of annulus fibrosus of canine intervertebral disc, of $1 \mathrm{~mm}$ height and $4 \mathrm{~mm}$ diameter. Details of these measurements can be found in chapter 5. The experiments consisted of four stages, the conditioning, swelling, compression, and control stages (Figures 1.7b, 3.4a). The duration of each stage was $6 \mathrm{~h}$ (sufficiently long in order to reach equilibrium), so that the total experiment time was $24 \mathrm{~h}$. The mechanical and chemical loads for both experiments are tabulated in table 3.1. Stage 1 is the conditioning stage. The sample is equilibrated from an unknown initial state to the conditioning equilibrium $(\approx 0.0775 \mathrm{MPa}, \approx 0.46 \mathrm{M})$. 


\begin{tabular}{|c||c|c||c|c||c|c||c|c||}
\hline \multicolumn{1}{|c||}{} & \multicolumn{2}{c||}{ conditioning } & \multicolumn{2}{c||}{ swelling } & \multicolumn{2}{c||}{ compression } & \multicolumn{2}{c|}{ control } \\
\hline exp. & W (MPa) & $\mathrm{C}(\mathrm{M})$ & W (MPa) & $\mathrm{C}(\mathrm{M})$ & W $(\mathrm{MPa})$ & $\mathrm{C}(\mathrm{M})$ & W (MPa) & $\mathrm{C}(\mathrm{M})$ \\
\hline $\csc 1$ & 0.0772 & 0.460 & 0.0772 & 0.159 & 0.193 & 0.159 & 0.0772 & 0.460 \\
\hline $\csc 2$ & 0.0778 & 0.461 & 0.0778 & 0.151 & 0.194 & 0.151 & 0.0778 & 0.461 \\
\hline
\end{tabular}

Table 3.1: The mechanical (W) and chemical loads ( $\mathrm{C}: \mathrm{M}=\mathrm{mol} / \mathrm{l}$ ) of $2 \mathrm{CSC}$ experiments, csel and csc2.

Then the sample is swollen by decreasing the external solution ionic strength to $\approx 0.15$ M. Due to time needed for the unmixing of the external salt concentration in the filter, 1 he change in external salt concentration is not taken as a step change in the simulation: an exponential function for the decrease or increase of the external salt solution with a time constant of $50 \mathrm{~s}$ is assumed. At the end of the swelling stage the sample is compressed during the compression stage by increasing the mechanical load to $\approx 0.19 \mathrm{MPa}$. Finally, in the control stage the same loading conditions are applied as in the conditioning stage.

The fit procedure The swelling and compression stages were filted by trial and error. c 14 elements were used. The duration of the time steps was $5 \mathrm{~s}$ for the first half hour of each phase, and after that $50 \mathrm{~s}$. Using longer time steps than $50 \mathrm{~s}$ resulted in changes in the transients, so it is advised to limit the length of the time step to $50 \mathrm{~s}$.

Constants in the calculations were: ${ }^{45}$

$\mathrm{Na}^{+}, \mathrm{Cl}^{-}$, and water molar masses: $M^{+}=22.99 \mathrm{~g} / \mathrm{mol}, M^{-}=35.45 \mathrm{~g} / \mathrm{mol}, M^{J}=18.00$ $\mathrm{g} / \mathrm{mol}$.

water concentration: $c^{f}=55.0 \mathrm{M}$. RT $=2.436^{*} 10^{3} \mathrm{~J} \mathrm{~mol}{ }^{-1}(\mathrm{~T}=293 \mathrm{~K})$.

The solid density, $\rho^{s}$, had been determined from the CSC experiments. The procedure by which this is done, is described in chapter 5 . The respective values were: experiment csc1: $\rho^{s}=0.00142 \mathrm{~g} / \mathrm{mm}^{3}$, experiment csc2: $0.00252 \mathrm{~g} / \mathrm{mm}^{3}$. For lack of data, the activity coefficient for the water, $\gamma^{f}$, was chosen constant. For both internal solution and external bath $\gamma^{f}$ equaled unity, which is valid for dilute solutions.

For the experiments' conditioning equilibrium, values for the liuid fraction, $\phi_{0}^{f}$, and the fixed charge density, $c_{0}^{p g}$, were determined from measurements of fixed charges and fluid content. These values were fixed input values for the fit procedure. Deformation dependencies (3.8) and (3.9) were used to calculate the values of $c^{p / 5}$, and $\phi^{S}$, respectively, during swelling and compression. The average ions activity coefficient, $\gamma^{ \pm}$, a parameter that also determines the equilibrium heights, was calculated automatically using the semi-empirical formulae of Manning. ${ }^{121}$ Because the values of $\gamma^{ \pm}$were estimated iteratively, concurrently with the ions concentrations, the starting value, $\gamma_{0}^{\frac{1}{0}}$, had no influence on the equilibrium values of this parameter (see chapter 5 ). Because of material loss that occurred in some degree during the experiments, a parameter that accounted for the loss of fixed charges, $\frac{d c^{p g}}{d t}$, was included. A linear loss of fixed charges after the onset of the swelling stage (in these two experiments at $6 \mathrm{~h}$ ) was assumed. The values for the effective stiffness, $H_{\epsilon}$, and the ratio of the stress free height of the sample, and the conditioning equilibrium height, $J_{s j, 0}{ }^{d}$, were determined from the fits.

\footnotetext{
${ }^{c} \ln$ rhapter 5 the fitting of CSC experiments is also described.

"Note that the subscript " 0 " is used to indicate the conditioning stage of the CSC experiments, as well as the reference state in the numerical formulation. The reference state in a CSC experiment is the
} 
The fit was performed as follows. First, the equilibrium heights of the swelling and compression stages were fitted by adjusting the stress free height ratio, $J_{s f, 0}$, effective stiffness, $H_{e}$, and fixed charges loss parameter, $\frac{d c^{p g}}{d t}$. Then, the compression transient was fitted by adjusting the permeability coefficient. It appeared that the swelling transient (II) could not be fitted correctly concurrently with the compression transient (III). Attention was focused on the correct fitting of the compression curve. The parameter values are shown in table 3.2 for both experiments.

\begin{tabular}{|c|c|c|c|c|c|}
\hline exp. & $c_{0}^{p g}(\mathrm{moleq} / \mathrm{l})$ & $\frac{d N^{p g}}{d t}\left(\mathrm{moleq} /\left(\mathrm{l}^{*} \mathrm{~h}\right)\right)$ & $\phi_{0}^{\prime}$ & $J_{s f, 0}$ & $H_{e}(\mathrm{MPa})$ \\
\hline $\mathrm{cscl}$ & 0.0848 & $-0.139^{*} 10^{-3}$ & 0.72 & 1.070 & 0.89 \\
\hline $\mathrm{csc} 2$ & 0.114 & 0 & 0.83 & 1.051 & 1.02 \\
\hline
\end{tabular}

Table 3.2: The parameter values for the $\operatorname{CSC}$ fit: $c_{0}^{p g}$ and $\phi_{0}^{\prime}$ were determined directly from measurements. $\frac{d e^{p s}}{d t}, J_{s f_{1} 0}$, and $H_{e}$ were determined in the fit procedure by trial and error.

For $K_{t r i}, D_{t r i}$ and $\gamma^{t}$ the values for the equilibrium states of the swelling and compression stages are given in table 3.3. In Figure 3.4 the results from the fits of experiments $\csc 1$ (a) and $\csc 2(\mathrm{~b})$ are depicted.

\begin{tabular}{|l||c|c||c|c||c|c|}
\hline \multicolumn{1}{|l||}{} & \multicolumn{2}{c||}{$K_{\text {tri }}\left({ }^{*} 10^{-16} \mathrm{~m}^{4} / \mathrm{Ns}\right)$} & \multicolumn{2}{c||}{$D_{\text {tri }}\left({ }^{*} 10^{-7} \mathrm{~m}^{2} / \mathrm{s}\right)$} & \multicolumn{2}{c|}{$\gamma^{ \pm}$} \\
\hline exp. & swel. & compr. & swel. & compr. & swel. & compr. \\
\hline csc1 & 2.09 & 1.36 & 3.45 & 2.93 & 0.698 & 0.691 \\
\hline csc2 & 2.92 & 2.36 & 5.27 & 4.91 & 0.687 & 0.682 \\
\hline
\end{tabular}

Table 3.3: The values of $K_{\text {tri }}, D_{t r i}$, and $\gamma^{ \pm}$for the equilibrium states of the swelling and compression stages determined from the computer simulation.

In both Figures $3.4 \mathrm{a}$ and $3.4 \mathrm{~b}$ the compression phases (III; from $12 \mathrm{~h}$ to $18 \mathrm{~h}$ ) are shown to be fitted excellently: there is no distinction visible between the fit (dashed line) 

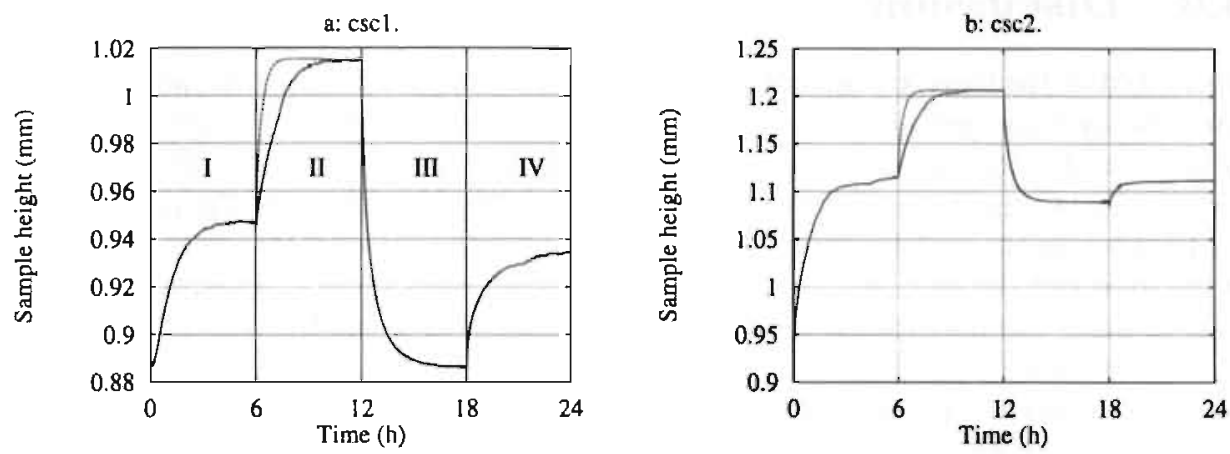

Figure 3.4: CSC fits of the swelling and compression phases of two CSC experiments, cscl and csc2. The solid line shows the experiment, the dashed line the fit. In (a) the 4 stages of the CSC experiment are indicated: $\mathrm{I}=$ conditioning, $\mathrm{II}=$ swelling, III $=$ compression, $\mathrm{IV}=$ control.

and the experiment (solid line). However, the transients of the swelling phase show that the fit rises faster than the experimental curve. The diffusion coefficients had to be chosen many times higher than the value of $D_{f r c c}^{\mathrm{NaCl}}\left(\right.$ at $25^{\circ} \mathrm{C}$ ). In the simulation the value $1^{*} 10^{-7}$ $\mathrm{m}^{2} / \mathrm{s}$ for $D_{f r e e}^{N a C l}$ was chosen (resulting in the equilibrium values shown in table 3.3 ), which is 62 times the real value of $D_{f r e e}^{\mathrm{NaCl}}$ in an aqueous solution $\left(1.61^{*} 10^{-9} \mathrm{~m}^{2} / \mathrm{s}\right)$.

Choosing the values of $D_{\text {free }}^{\mathrm{NaCl}}$ for the fit still higher had no effect in terms of slowing down the swelling response in the fit. This could only be achieved by reducing the value of the permeability coefficient. Reducing $K_{t r i}$ to fit the swelling transient, still resulted in too high values of $D_{t r i}$, and deteriorated the fit for the compression stage.

Further results of CSC: experiment fits are given in chapter 5. 


\subsection{Discussion}

This FEM of the three phase mixture theory for swelling tissues is to the author's knowledge the first one with degrees of freedom that are continuous at the boundaries. The advantage of a formulation with continuous degrees of freedom is that there is no need for interface elements, contributing greatly to the user-friendliness of the program.

In choosing the continuous dofs one could consider the choice of the chemical potentials of the fluid and the ions, and not expressions with e-powers thereof. This, however, would lead to more complex forms, because of the incidence of gradients and time derivatives in the formulae.

In the current formulation the Lagrangian forms of the permeability and diffusion tensors, $\boldsymbol{K}^{*}$ and $\boldsymbol{D}^{*}$, are used. This is important for the objectivity of these tensors: their values should not be influenced by rigid rotations. This is ensured by the total Lagrange formulation. However, deformation dependency of these tensors should be accounted for. In our 1D implementation we chose for relationships coupling the $K_{t r i}$ and $D_{\text {tri }}$ to the fluid content. Both relationships are based on experimental results.

The 1D computer model was used for the simulation of the swelling and compression phases of two CSC experiments. It was possible to fit the equilibria of the swelling and compression phases, by adjusting the stress free height and solid stiffness of the samples. The parameter for the decrease of fixed charge density was used to account for continuous loss of height in the compression phase, and proved a valuable parameter in fitting the compression phase of experiment 1 .

The compression transient was fitted by adjusting the permeability coefficient. Very good fits of the compression phase were obtained in this way. The values of the permeability coefficients $\left((1.36-2.92)^{*} 10^{-16} \mathrm{~m}^{4} / \mathrm{Ns}\right)$ are in the same range as those found by Urban and Maroudas: $\left.{ }^{222}(0.5-11.5)^{*} 10^{-16} \mathrm{~m}^{4} / \mathrm{Ns}\right)$. The values of the average ionic activity coefficients $(0.682-0.698)$ are in the range of values for cartilage $(0.65-0.72)$ reported by Maroudas. ${ }^{128}$

Although the experiments and fits show excellent agreement for the equilibria and compression transients, there were problems with the swelling transient (II; from $6 \mathrm{~h}$ to $12 \mathrm{~h}$ ). The diffusion coefficient had to be chosen so high that further increase in $D_{t_{r i}}$ did not result in further decrease in swelling speed of the fit. Even decreasing $K_{\text {iri }}$ which resulted in a poorer fit of the compression phase, did not yield acceptable values for $D_{\text {tri }}$, i.e., values smaller than the diffusion coefficient of $\mathrm{NaCl}$ in free water, although then it was possible to fit the swelling transient. This finding is consistent with data of Snijders. ${ }^{200}$ Oomens et al. ${ }^{163}$ show a similar conclusion for swelling of synthetic hydro-gel. Therefore, we conclude that the triphasic model, on which this formulation is based, is inadequate to simulate swelling phenomena with a correct diffusion coefficient.

Summarizing, the 1D computer implementation of the triphasic model performs adequately for equilibrium situations and compression transients. However, for swelling the triphasic model appears to overestimate the diffusion coefficient. Although a $1 \mathrm{D}$ computer implementation with continuous dofs has been proven to work, no comparison of convergence rates with and without boundary elements has been conducted. Further research should focus on the convergence rates and 2- and 3D generalization of the present work, combining a numerical formulation with continuous dofs with an adaptation of the triphasic theory that models swelling adequately. 


\subsection{Appendix}

In this appendix we give the deviations of $p, \xi, \beta$, and $\gamma$, as a function of deviation in the dofs $u, \epsilon_{p}$, and $\epsilon_{c}$. In order to write the variables only as functions of the dofs, we have to write $\delta c^{p g}$ and $\delta \phi^{f}$ also as functions of them. We first repeat some definitions of variables, and then give their deviations.

$$
\begin{gathered}
c_{ \pm}^{f}=\frac{2 c^{f}}{\gamma^{ \pm}} . \\
c^{p g}=\frac{c_{0}^{p g}}{1-\frac{1-J}{\phi_{0}^{J}}} . \\
\phi^{f}=1-\frac{1-\phi_{0}^{f}}{J} . \\
c^{\kappa}=\sqrt{\left(c^{p g}\right)^{2}+\left(\frac{2 c^{f}}{\gamma^{ \pm}}\right)^{2} \epsilon_{c} .} \\
\delta \phi^{f}=\frac{1-\phi_{0}^{f}}{J} \boldsymbol{F}^{-1}:\left(\nabla_{0} \delta \boldsymbol{u}\right)^{c} . \\
\delta c^{p g}=-\frac{J\left(c^{p g}\right)^{2}}{\phi_{0}^{f} c_{0}^{p g}} \boldsymbol{F}^{-1}:\left(\nabla_{0} \delta \boldsymbol{u}\right)^{c} . \\
-\frac{\left(c^{p g}\right)^{3} J}{\phi_{0}^{f} c_{0}^{p g}} \boldsymbol{F}^{-1}:\left(\nabla_{0} \delta \boldsymbol{u}\right)^{c}+\frac{1}{c^{\kappa}}\left(\frac{1}{2}\left(c_{ \pm}^{f}\right)^{2} \delta \epsilon_{c} .\right.
\end{gathered}
$$

The pressure $p$ is given by:

$$
p=c^{f} R T\left[\ln \epsilon_{p}-\ln \gamma^{f} x^{f}\right]=c^{f} R T \ln \left[\frac{\epsilon_{p}}{\gamma^{f}}\left(1+\frac{c^{\kappa}}{c^{f}}\right)\right] .
$$

The deviation in $\mathrm{p}$ is given by:

$$
\delta p=p_{1} J \boldsymbol{F}^{-1}:\left(\nabla_{0} \delta \boldsymbol{u}\right)^{c}+p_{2} \delta \epsilon_{p}+p_{3} \delta \epsilon_{c},
$$

with:

$$
\begin{array}{r}
p_{1}=-R T \Phi^{f} \frac{\left(c^{p g}\right)^{3}}{c^{\kappa}\left(1+\frac{c^{\kappa}}{c^{j}}\right) \phi_{0}^{f} c_{0}^{p g}}, \\
p_{2}=R T \frac{c^{j}}{c_{p}}, \\
p_{3}=R T \Phi^{f} \frac{\left(c_{ \pm}^{f}\right)^{2}}{2 c^{\kappa}\left(1+\frac{c^{\kappa}}{c^{j}}\right)},
\end{array}
$$

with:

$\Phi^{\prime}=1+\frac{\partial \ln \gamma^{f}}{\partial \ln x^{\prime}}$ 
$\xi$ is given by:

$$
\xi=\frac{1}{2}\left(M^{d i J} c^{p g}+M^{ \pm} c^{\kappa}\right) \frac{1}{M^{ \pm} c^{\prime}} .
$$

The deviation in $\xi$ is given by:

$$
\delta \xi=\xi_{1} \boldsymbol{F}^{-1}:\left(\nabla_{0} \delta \boldsymbol{u}\right)^{c}+\xi_{2} \delta \epsilon_{c},
$$

with:

$$
\begin{array}{r}
\xi_{1}=-\frac{\left(c^{p g}\right)^{2} J}{2 c^{f} \phi_{0}^{f} c_{0}^{p g}}\left(\frac{M^{d i f}}{M^{ \pm}}+\frac{c^{p g}}{c^{\kappa}}\right), \\
\xi_{2}=\frac{\left(c_{ \pm}^{j}\right)^{2}}{4 c^{f} c^{\kappa}} .
\end{array}
$$

$\beta$ is given by:

$$
\beta=\frac{M^{ \pm}\left(c_{ \pm}^{f}\right)^{2}}{4 c^{\kappa}}
$$

The deviation in $\beta$ is given by:

$$
\delta \beta=\beta_{1} \boldsymbol{F}^{-1}:\left(\nabla_{0} \delta \boldsymbol{u}\right)^{c}+\beta_{2} \delta \epsilon_{c},
$$

with:

$$
\begin{array}{r}
\beta_{1}=\frac{M^{ \pm}\left(c_{ \pm}^{f}\right)^{2}\left(c^{p y}\right)^{3} J}{4 \phi_{0}^{j} c_{0}^{p g}\left(c^{\kappa}\right)^{3}}, \\
\beta_{2}=-\frac{M^{ \pm}\left(c_{ \pm}^{j}\right)^{4}}{8\left(c^{\kappa}\right)^{3}} .
\end{array}
$$

$\gamma$ is given by:

$$
\gamma=\frac{1}{2}\left(M^{d i f}+\frac{M^{ \pm} c^{p g}}{c^{n}}\right) .
$$

The deviation in $\gamma$ is given by:

$$
\delta \gamma=\gamma_{1} \boldsymbol{F}^{-1}:\left(\nabla_{0} \delta \boldsymbol{u}\right)^{c}+\gamma_{2} \delta \epsilon_{\varepsilon},
$$

with:

$$
\begin{gathered}
\gamma_{1}=\frac{M^{ \pm} J c^{p g}}{2 c_{0}^{p g} \phi_{0}^{f} c^{\kappa}}\left(\frac{c^{p g}}{c^{\kappa}}-1\right), \\
\gamma_{2}=-\frac{M^{ \pm}\left(c_{ \pm}^{f}\right)^{2} c^{p g}}{4\left(c^{\kappa}\right)^{3}} .
\end{gathered}
$$

The time derivatives of $c^{p g}$ and $\delta c^{p g}$ are given by:

$$
\begin{gathered}
\left(c^{\dot{p} g}\right)_{s}=-\frac{c_{0}^{p g}}{\phi_{0}^{J}} \frac{(\dot{J})_{s}}{\left(1-\frac{1-J}{\phi_{1}^{J}}\right)^{2}}, \\
\left(\delta \dot{c}^{p g}\right)_{s}=\frac{c_{0}^{p g}}{\phi_{0}^{J}}\left[\frac{2(j)_{s}\left(c^{p g}\right)^{3} J}{\phi_{0}^{J}\left(c_{0}^{p g}\right)^{3}} \boldsymbol{F}^{-1}:\left(\boldsymbol{\nabla}_{0} \delta \boldsymbol{u}\right)^{c}\right. \\
\left.+\frac{\left(c^{p g}\right)^{2}}{\left(c_{0}^{p g}\right)^{2}}\left((j)_{s} \boldsymbol{F}^{-1}:\left(\boldsymbol{\nabla}_{0} \delta \boldsymbol{u}\right)^{c}+J\left(\boldsymbol{F}^{-1}\right)_{s}:\left(\nabla_{0} \delta \boldsymbol{u}\right)^{c}+J \boldsymbol{F}^{-1}:\left(\boldsymbol{\nabla}_{0}(\delta \boldsymbol{u})_{s}\right)^{c}\right)\right] .
\end{gathered}
$$


Chapter 4

Non-homogeneous permeability of canine anulus fibrosus 


\subsection{Summary}

Study design. This paper examines the permeability coefficient and aggregate modulus of slices of annulus cut from canine lumbar intervertebral discs.

Objectives. The purpose was to examine the influence of radial position on these material properties, including outer samples with intact annulus edge.

Summary of background data. The outer edge of annulus fibrosus shows radial bulge during axial compression of motion segments. The radial bulge increases monotonically when the axial compression is sustained for several hours, until a plateau is reached. Triphasic modeling of axial compression shows that this time course of radial bulge can not be obtained using a uniform permeability coefficient according to literature values.

Methods. Confined compression experiments (controlled load) were designed to measure the time course of uni-axial deformation of samples of diameter $4 \mathrm{~mm}$, and height $1 \mathrm{~mm}$. The rotation symmetry axis of the samples was taken in the radial direction of the disc. The radial permeability coefficient as well as the aggregate modulus were determined by fitting the compression data using linear biphasic theory.

Results. The permeability coefficient was lower at the periphery than in deeper layers of the annulus. Outer samples with outer surface 0.0 to $0.5 \mathrm{~mm}$ from the annulus edge had an average permeability coefficient of $(1.02 \pm 0.57)^{*} 10^{-16} \mathrm{~m}^{4} / \mathrm{Ns}(\mathrm{n}=24)$. Inner samples at 2.0 to $2.5 \mathrm{~mm}$ had an average permeability coefficient of $(2.81 \pm 0.98)^{*} 10^{-16}$ $\mathrm{m}^{4} / \mathrm{Ns}(\mathrm{n}=13)$. The aggregate modulus $H_{A}$ of outer samples was significantly higher $\left(H_{A}=1.56 \pm 0.34 \mathrm{MPa}\right)$ than that of inner samples $\left(H_{A}=1.31 \pm 0.47 \mathrm{MPa}\right)$.

Conclusions. The fact that the outer annulus is less permeable than the inner may explain why radial bulge of annulus fibrosus increases monotonically in time to an equilibrium value during sustained axial compression of a motion segment. [Key words: annulus fibrosus, intervertebral disc, permeability, biphasic]

Accepted by Spine (publication in January 1997) with co-authors: Maarten R. Drost, Jacques M. Huyghe, Jan D. Janssen, and Anthony Huson. 


\subsection{Introduction}

The intervertebral disc (IVD) consists of collagen fibrils and fibres embedded in a proteoglycan network. In the central part of the disc, the nucleus, the collagen fibrils are randomly oriented, whilst in the annulus the fibres are organized in lamellae, enveloping the nucleus. The fibre direction alternates from lamella to lamella, and makes angles of about $65^{\circ}$ or $-65^{\circ}$ with the axis of the spine. Bottom and top of the IVD are bordered by the end plates, to which the inner lamellae of the annulus attach. The hydration of the disc decreases with age: for the nucleus from $88 \%$ at birth to $65 \%$ at 77 years, for the annulus from $78 \%$ at birth to $70 \%$ at 30 years ${ }^{156,175}$ (all percentages by weight).

For the mechanical functioning of the disc both the biochemical composition and the structural organization are important. Biochemical composition depends on age, level in the spine, region in the disc, pathology (e.g. herniation, scoliosis). A comprehensive review on the biochemical composition, structure and mechanical functioning is given by Ghosh. ${ }^{62}$

For cartilage, research has been done on the permeability coefficient a as a function of distance from the articular surface. ${ }^{125,128,149}$ The permeability coefficient was found to increase toward the articular surface. In some cases there was an increase of the permeability coefficient from the deeper zones to the superficial layers and then a decrease to the articular surface. Measurements of the permeability coefficient as a function of fixed charge density (FCD) and collagen contents yielded an inverse relationship for both: for FCD, ${ }^{125}$ and for FCD and collagen. ${ }^{149}$

Mansour and Mow ${ }^{122}$ measured the permeability coefficient in bovine cartilage as a function of compressive strain and high pressures. They found that the permeability coefficient decreased linearly with the strain, and non-linearly with the pressure.

To date, little has been published about the permeability coefficient of the IVD. Urban and Maroudas ${ }^{222}$ determined a biphasic permeability coefficient of human radial annulus samples, and axial nucleus samples, and gave the variation of $K_{b i}$ with hydration for one disc. They found that $K_{b i}$ rose with hydration. Best et al. ${ }^{17}$ determined the biphasic permeability coefficient $K_{b i}$ from confined compression experiments of human L3-4, and L4-5 discs. They found no differences in $K_{b i}$ for different regions and radial positions in the disc. Drost et al. ${ }^{51}$ tested canine discs, also in confined compression experiments ( $\mathrm{L} 4$ $5, \mathrm{~L} 5-6, \mathrm{~L} 6-7, \mathrm{~L} 7-\mathrm{Sl}$ ), and found no significant differences in the permeability coefficient for different regions in the disc. They didn't test the radial dependence of $K_{b i}$.

Using the triphasic chemo-mechanical theory of Lai et al., ${ }^{109} \mathrm{Gu}$ and coworkers ${ }^{71}$ determined an expression for the permeability coefficient, which was used in the simulation of a one-dimensional permeation experiment. Their permeability law sustains an increase of the permeability coefficient with increasing hydration and decreasing FCD. Snijders, ${ }^{200}$ in his triphasic model of cartilaginous tissues uses an exponential function for the deformation dependency of $K_{\text {tri }}$ defining an increasing $K_{\text {tri }}$ with increasing hydration.

In the IVD there are two routes for fluid exchange: via the annulus and the end

${ }^{\mathrm{a}}$ Generally, the symbol $K$ is used for the permeability coefficient, which has different definitions. In this chapter the biphasic $K_{b i}$ is determined. Whenever reference is made to the permeability coefficient in this chapter, the $K_{b i}$ is meant. The permeability coefficient defined in the numerical formulation in chapter 3 of this thesis has the symbol $K$, whereas the triphasic permeability coefficient is designated with the symbol $K_{t r i}$. For a more detailed explanation, see chapter 3. 
plates, as diffusion studies have shown, e.g. ${ }^{152,158,159,218}$ Fluid flow along these pathways is governed by mechanical and osmotic pressure gradients in the disc and its permeability coefficient. E.g., under axial compression, permeability influences the time course of amplitude of axial deformation and radial disc bulge. Experiments on motion segments that were axially compresse $\mathrm{d}^{67}$ performed in our laboratory showed a monotonically increasing radial bulge up to a time of $4 \mathrm{~h}$. The computer simulation using the numerical model of Snijders et al. ${ }^{203}$ calculated an initial increase of bulge to a local maximum after about $1 \mathrm{~h}$, followed by a decrease to an equilibrium value after $4 \mathrm{~h}$. For the simulation the same $K_{t r i}$ for both nucleus and annulus (constant over the entire disc) was used. The $K_{\text {tri }}$ was deformation dependent with an initial value of $9^{*} 10^{-16} \mathrm{~m}^{4} / \mathrm{Ns}$. This value was based on measurements of Urban and Maroudas ${ }^{22}$ who found $K_{6 i}$ to lie in the range of $(0.5-11.5)^{*} 10^{-16} \mathrm{~m}^{4} / \mathrm{Ns}$. Other material parameters were also derived from literature data. The discrepancy in measured and simulated results motivated further study of the constitutive properties of the disc in vitro. In particular the magnitude of the hydraulic permeability as a function of radial position in the disc was thought to play a role in the explanation of the differences found between the simulation and the experiment of the radial bulge.

A possible reason for the existence of an inhomogeneous permeability coefficient in the annulus fibrosus might be that the outer annulus, which according to our information had never been measured with intact edge, has a lower permeability than the rnore inner regions. It was therefore the goal of the present study to investigate the radial variation in the permeability coefficient in the annulus fibrosus. 


\subsection{Methods}

\subsubsection{Sample preparation}

The lower lumbar spines of 7 dogs ( 2 males, 5 females; races were shepherd, bouvier des Flandres, and bobtail) were obtained postmortem. See also Drost et al., ${ }^{31}$ who performed similar studies. Body weights ranged 22-26 kg. Ages at death ranged from 1 to 4 years. Within 1-3 h after death the spines were dissected. Spines were sawed off above the lumbar region, and below L7-S1. The ventral sides of the discs were partially freed of muscle tissue, and the longitudinal anterior ligament, leaving the surface of the discs intact for measurements. After dissection, the spines were sealed in plastic bags, and kept frozen at $-65^{\circ} \mathrm{C}$.

Within two and a half weeks, the frozen spines were sawed, excising in radial direction parts of the discs in more or less rectangular slabs, of dimensions of roughly
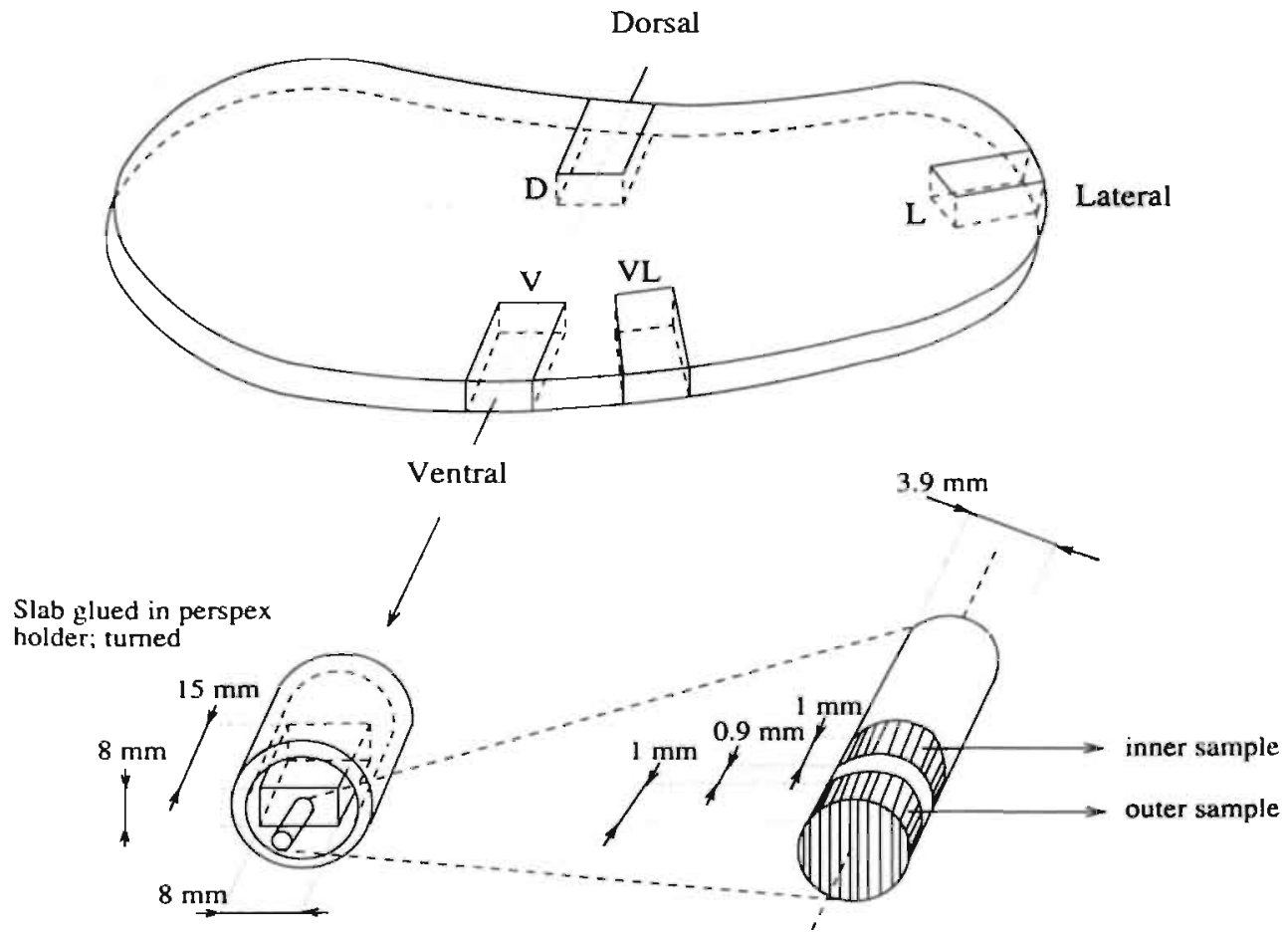

Figure 4.1: Preparation of the samples. Slabs are sawed out of frozen discs, glued in cylindrical holders, and finally turned on a lathe.

$15($ radial $) * 8$ (circumferential) $* 8$ (axial) $\mathrm{mm}^{3}$. The slabs were from discs L7-S1 to L4-5, and from either of the ventral (V), ventrolateral (VL), or dorsal (D) regions (Figure 4.1). The length axes of the slabs were approximately perpendicular to the outer surface of the anuulus. 
Within 9 days the slabs were glued into perspex cylindrical holders of inner diameter 10.5 $\mathrm{mm}$, or $11.7 \mathrm{~mm}$, with outer diameter $15.0 \mathrm{~mm}$, and height of $25-30 \mathrm{~mm}$, with a closed bottom. In the exceptional case of very small slabs $(n=3)$ they were glued in holders of inner diameter $9.0 \mathrm{~mm}$, outer diameter $10.0 \mathrm{~mm}$ and height $25-30 \mathrm{~mm}$. The slabs were glued with the outer surface of the annulus perpendicular to the axis of the holders, the surface sticking out about $1 \mathrm{~mm}$. Tissue-tek (OCT Compound 4583, Miles Diagnostics, Elkhart, IN, USA) was used to glue the slabs. During gluing, the slabs were kept frozen, using liquid nitrogen $\left(-196{ }^{\circ} \mathrm{C}\right)$. Upon contact with the liquid nitrogen, the tissue-tek solidified.

In case the surfaces of the ventral slabs were polluted with blood, their surfaces were scraped clean with a scalpel, taking care not to damage them. The same was done with dorsal slabs of which roughly $50 \%$ contained remnants of the spinal cord that had not been broken off cleanly during sawing, or blood stains.

Within 3 days the glued slabs were turned on a lathe. Radial samples of approximately $1 \mathrm{~mm}$ height with a $3.9 \mathrm{~mm}$ diameter were turned keeping the chisel and slabs cooled with liquid nitrogen. When possible 2 neighbouring samples per slab, with an interspace (lost material from the thickness of the chisel) of $0.9 \mathrm{~mm}$ were produced. The outer samples were either with intact outer annulus surfaces, or these were flattened on the lathe. Loss of material for flattening the outside surface of outer samples amounted to approximately $0.5 \mathrm{~mm}$. The inner samples were flattened on both sides on the lathe.

Thus, we get 4 categories of samples:

1. Outer samples with intact outer surface (osi) at $0.0 \mathrm{~mm}$ from the annulus edge ( $\mathrm{n}=$ 15),

2. Outer samples with non-intact, flattened outer surfaces (osf) at approximately $0.5 \mathrm{~mm}$ from the annulus edge $(n=9)$,

3. Inner samples neighbouring outer samples with an intact outer surface (isi) at approximately $2.0 \mathrm{~mm}$ from the annulus edge $(n=10)$,

4. Inner samples neighbouring outer samples with a flattened outer surface (isf) at approximately $2.5 \mathrm{~mm}$ from the annulus edge $(\mathrm{n}=3)$.

The samples were put in aluminum cups and stored to a naximum of 17 days before being used in the experinent. During all the stages of preparation the samples had been kept frozen, using liquid nitrogen as a coolant. In total $3 i$ samples of the 74 were successfully tested.

\subsubsection{Confined swelling and compression setup}

Three identical measurement setups were used in parallel. In Figure 4.2a the setup is given schematically. A description of the measurement apparatus is also 
a.

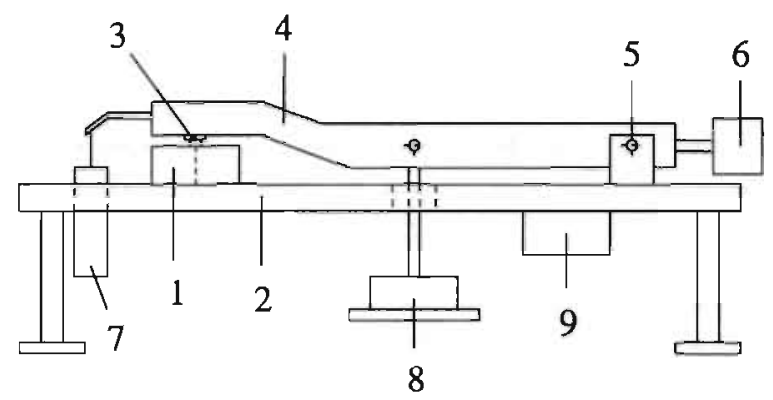

b.

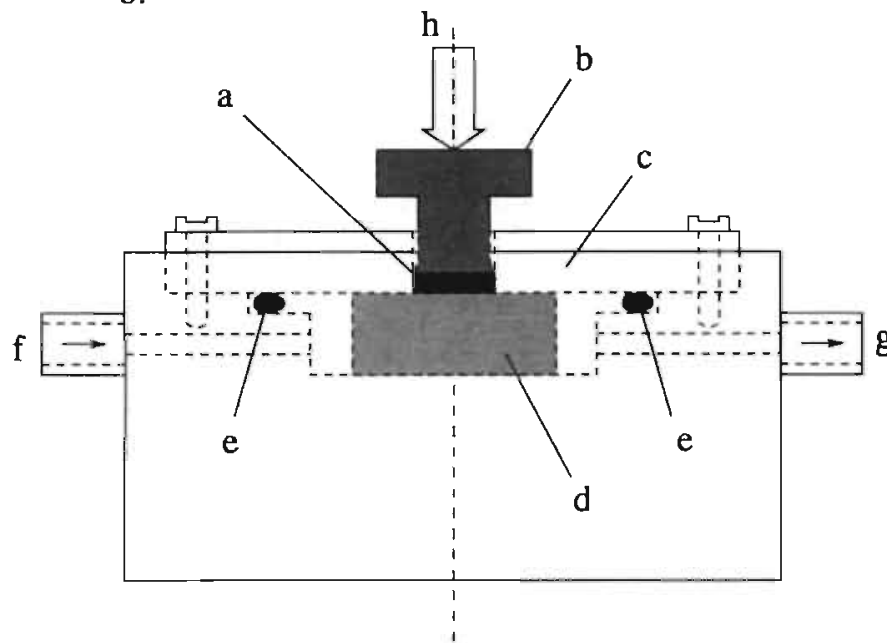

Figure 4.2: Schematic view of the measurement setup (a), with detail of the measurement chamber (b). (a) setup; 1: measurement chamber, 2: table, 3: piston, 4: loading arm, 5: rotation axis, 6 : counter weight, 7 : linear displacement transducer, 8: weight, 9: vibrator. (b) measurement chamber; $a$ : sample, b: piston, $c$ : cover platen, $d$ : filter, e: O-ring, f: fluid inlet, $\mathrm{g}$ : fluid outlet, $\mathrm{h}$ : mechanical load.

(Scientific Solutions Inc., Solon, Ohio, U.S.A.) to an IBM-AT. A vibrator was attached to the setup to overcome sticking of the sample and piston to the chamber wall. $0.5 \mathrm{~s}$ after each sample point, the setup was vibrated at $50 \mathrm{~Hz}$ during $2 \mathrm{~s}$. The data acquisition sampling frequency was $0.125 \mathrm{~Hz}$.

\subsubsection{Experimental protocol}

To allow for free movement of the piston the measurement chamber was placed on a film of silicon oil, and the circumference of the piston greased with vaseline. Using a scalpel, the frozen sample was freed of irregularities that resulted from the turning. The sample was then placed in the chamber, and the piston placed on top. The sample was allowed to thaw while the salt solution was circulated through the filter. During the thawing the given in Snijders, ${ }^{200}$ and Drost et al. ${ }^{51}$ The heart of the experimental setup for the confined swelling and compression measurements consisted of a cylindrical stainless steel chamber of diameter $4.030 \pm$ $0.004 \mathrm{~mm}$. The bottom of this chamber was formed by a sintered glass filter (pore sizes 16-40 $\mu \mathrm{m}$, permeability coefficient $10^{-12}$ $\mathrm{m}^{4} / \mathrm{Ns}$ ). The top of the chamber was closed off by a piston, see Figure 4.2b.

Mechanical loading of the samples was accomplished by a cantilever arm on which the loading piston was mounted. Weights were hung on this arm, the compressive load of which was transferred via the piston. Chemical loading was done by flowing a bathing solution of $\mathrm{NaCl}$ through the filter. The loading arm was connected to a DC operated linear variable displacement transducer (LVDT, Schaevitz) interfaced by a Labmaster 12 bit $\mathrm{AD}$ converter 
sample automatically expanded against the wall, filter and piston. Data acquisition was started. The temperature was kept at $21 \pm 1{ }^{\circ} \mathrm{C}$.

The load scheme consisted of 3 stages: the conditioning, compression, and control stages. For all stages the bathing solution had the same strength: a solution of 0.16 $\pm 0.01 \mathrm{M} \mathrm{NaCl}$, buffered with $5 \mathrm{mM}$ Tris at $\mathrm{pH} 7.9$ was used. A salt strength around the physiological had been chosen, because it is closest to the in vivo situation. The $\mathrm{pH}$ was kept higher than 6 , because in this range the fixed charge density in cartilage is independent of the $\mathrm{pH} .{ }^{128}$ We assume that this is also valid for intervertebral disc annulus. Unlike the experiments described by Drost et al., ${ }^{51}$ only the mechanical load was varied from one stage to another. This suffices for determination of the permeability coefficient. During the conditioning stage the sample was equilibrated against a mechanical load of 0.0772 , or $0.0778 \mathrm{MPa}$ (depending on the setup). In most cases the sample swelled during the conditioning stage, but in 6 out of the 37 cases (which were all outer samples) the sample decreased in height. The compression stage (used for the determination of the permeability coefficient) consisted of an increase of the mechanical load to $0.194 \mathrm{MPa}$, resulting in a decrease of sample height. Finally, in the control stage the sample was again loaded with 0.0772 or $0.0778 \mathrm{MPa}$, equaling the loading conditions to those in the conditioning stage. The changes of weights for the 2 nd and $3 \mathrm{rd}$ stages were done using a computer controlled step motor.

The total testing time was chosen as short as possible in view of possible tissue changes and loss of material (proteoglycans in the filter ${ }^{51}$ ) during the experiment. However, in order to reach equilibrium (i.e., a horizontal tangent in the time-(sample height) curve) in the conditioning stage, which is imperative for the determination of the permeability coefficients, the testing time of the conditioning stage had to be sufficiently long. It appeared that per stage the equilibration times were approximately the same. Equilibration times were influenced by the thickness of the sample. The thicker the sample, the longer the equilibration time. Also, outer samples with intact outer surfaces required longer equilibration times than non-intact outer samples or inner samples. The total testing time was either $30 \mathrm{~h}$ or $15 \mathrm{~h}$. For samples with intact outer surface (osi) and their neighbours (isi), a $30 \mathrm{~h}$ experimentation time was used. To maintain the same experimental conditions for their neighbours (isi), and because these samples were in general thicker than the isf samples, these were also tested for $30 \mathrm{~h}$.

For non-intact outer samples (osf) and their neighbours (isf), the experiment lasted $15 \mathrm{~h}$. For the $15 \mathrm{~h}$ experiments the duration per stage was $5 \mathrm{~h}$, except in three cases, where the conditioning stages lasted $4.5 \mathrm{~h}$ and the compression stages $5.5 \mathrm{~h}$. For the 30 $\mathrm{h}$ experiments the duration per stage was $10 \mathrm{~h}$.

\subsubsection{Data analysis}

The compression phases of the experiments were used to determine the biphasic permeability coefficients. The compression phase was fitted with a generalized, cross-validatory, cubic B-spline 235 to remove noise. The exact moment of the beginning of the compression phase was estimated visualiy from the raw data files. The end point of the compression phase was taken at $5 \mathrm{~h}$ aiter the start of this phase. This means that for the $15 \mathrm{~h}$ experiments with 5 h phases, the complete measured compression phase was used. For the 15 $\mathrm{h}$ experiments with $5.5 \mathrm{~h}$ compression measurement time, and for the $30 \mathrm{~h}$ experiments 
with $10 \mathrm{~b}$ compression measurement time thus not all the measurement data was used. This was motivated by the fact that after $15 \mathrm{~h}$ of experiment time height loss presumably due to loss of proteoglycans seemed to play a far more important role, than between 0 and $15 \mathrm{~h}$, as could be seen from the time-(sample height) curves. In a number of the 30 $\mathrm{h}$ experiments $(\mathrm{n}=9)$ an increase in the rate of height loss was noticed $15 \mathrm{~h}$ after the start of the experiment.

For an experiment to be used for permeability determination a number of selection criteria had to be met. Visual inspection of the sample had to be satisfactory, i.e. there had to be no bone fragments present, or nucleus material. Bone or nucleus material, especially the latter, were sometimes not visible when the sample was in a frozen state, before being used in an experiment. So, in some cases, results were discarded upon visual inspection after the experiment. Sometimes experimental problems (e.g. air in the fluid circuit, displacement outside the range of the AD converter) rendered the results unacceptable.

Furthermore, it was demanded that the conditioning phase was sufficiently equilibrated, so that the results of the different experiments were comparable. All compression phases had to start from the same equilibrated state $(0.16 \mathrm{M}, 0.0772$ or $0.0778 \mathrm{MPa})$. We discarded the experiment when the absolute value of the sample's average velocity of height change during the last hour of the conditioning phase was too high, because then the conditioning phase was not sufficiently equilibrated. This velocity was defined in terms of a \% of the average height of the last hour of the conditioning phase per hour. An upper limit of $0.89 \% / \mathrm{h}$ was chosen based on what was deemed a reasonable measure from visual inspection of the time-displacement plots. The mean \pm standard deviation of the velocity was: $0.22 \pm 0.32 \% / \mathrm{h}$. The mean velocity was determined by linear regression through the last hour of the phase.

In roughly $50 \%$ of the experiments that complied with the above-mentioned criteria, the compression phase was not at equilibrium after $5 \mathrm{~h}$. However, it was not a demand that the compression phase was equilibrated, because the displacement-time curve calculated with biphasic theory can also be fitted on a non-equilibrated curve. We thus fitted both equilibrated and unequilibrated compression phases, and used the equilibrium height of the biphasic fit as an estimate for the real equilibrium height after compression.

Finally, the relative least squares error in the determination of the compression time constant $\tau$ (see below) was required to be less than 5 percent. This relative error was defined as the average sum of squares between the fit and the raw data divided by $\tau$.

Errors due to sample impurities, experimental problems, and the non-equilibration criterion resulted together in the exclusion of 36 experiments. The demand of the maximum $5 \%$ error in the least squares fit for $\tau$ resulted in the exclusion of one experiment. This left 37 experiments eligible for permeability determination.

The sample height loss for any sampling point of the compression phase was normalized with respect to the total height loss of that phase:

$$
U(i)=\frac{h(i)-h\left(i_{\text {begin }}\right)}{-\Delta h} .
$$

with:

$U(i)=$ the normalized sample height for sampie point $\mathrm{i}$, 
$h(i)=$ the sample height for sample point $\mathrm{i}$,

$i_{\text {begin }}=$ the last plateau point before the compression phase,

$i_{\text {end }}=$ the last point of the compression phase,

$\Delta h=-\left(\mathrm{h}\left(i_{\text {begin }}\right)-\mathrm{h}\left(i_{\text {end }}\right)\right)=$ the total height loss for the compression phase.

The normalized compression phase was fitted with a non-linear least square method to the analytical solution of linear biphasic compression: ${ }^{211}$

$$
\begin{array}{r}
U=\frac{h(t)-h(t=0)}{-\Delta h_{f i t}}, \\
U(T)=\sum_{n=0}^{\infty} \frac{2}{M^{2}}\left(1-e^{-M^{2} T}\right), \\
T=\frac{t}{\tau} ; M=\frac{\pi}{2}(2 n+1),
\end{array}
$$

with:

$U=$ normalized sample height,

$h=$ sample height,

$\Delta h_{\text {fit }}=-(h(t=\infty)-h(t=0))$,

$T=$ normalized time,

$t=$ time since the beginning of the compression phase,

$\tau=$ compression time constant.

The time constant $\tau$ is dependent on the sample height $h_{s}$. For $h_{s}$ the height of the sample at the end of the conditioning phase (i.e. the heginning of the compression phase) was chosen because this was the best equilibrated phase: $h_{s}=\mathrm{h}\left(i_{\text {begin }}\right)$. In working out the equations of the biphasic theory, defining a linear elastic solid, it is found that:

$$
\tau=\frac{h_{s}^{2}}{H_{A} * K_{b i}},
$$

with:

$H_{A}=$ the compressive aggregate modulus,

$K_{b i}=$ the biphasic permeability coefficient.

The compressive aggregate modulus is defined as the quotient of total Cauchy stress $(\sigma)$ from the external load (= weight/surface) and Cauchy's infinitesimal strain $(\epsilon)$. The infinitesimal strain is taken as the quotient of the height difference of the compression phase fit $\Delta h_{\text {fit }}$ and the sample height after conditioning $h_{s}$.

$$
\begin{gathered}
H_{A}=\frac{\sigma}{\epsilon}, \\
\epsilon=\frac{\Delta h_{f i t}}{h_{s}} .
\end{gathered}
$$

From equations (4.3) and (4.4) the aggregate modulus $H_{A}$, and the permeability coefficient $\kappa_{b i}$ can be determined. 
Note that the height difference of the fitted compression phase $\Delta h_{f i t}$ does not have to equal the height difference $\Delta h$ of equation (4.1). In a number of cases (especially the $30 \mathrm{~h}$ experiments) the compression phase had not reached equilibrium after $5 \mathrm{~h}$. In these cases there is a difference between $\Delta h_{f i t}$ and $\Delta h$.

We used the non-parametric Mann-Whitney $U$, and the Wilcoxon matched pairs signed ranks statistics to test for differences between groups. Where there is no reference, the Mann-Whitney U test has been used. 


\subsection{Results}

For two of the experiments Figure 4.3 shows the curves of the sample height versus time. The fit of the compression stage is given by the smooth solid line. The sample's

a.

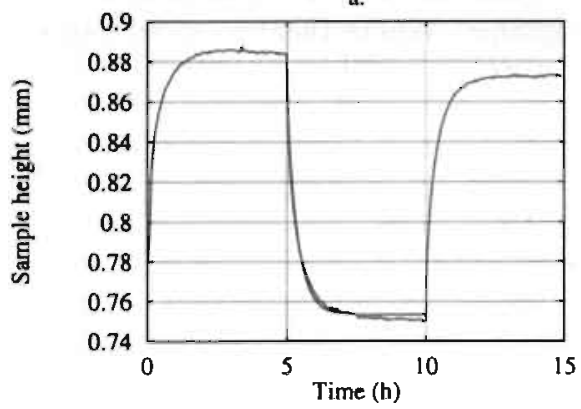

b.

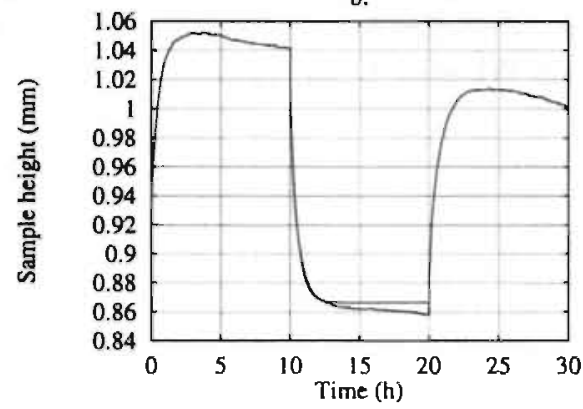

Figure 4.3: Sample height versus time for an osf (15 h) experiment (sample from outer regions of annulus with a flattened outer surface) (a), and an isi $(30 \mathrm{~h}$ ) experiment (sample from inner regions of annulus, neighbouring an outer sample with intact outer surface; b). The fit for the compression phase is given by the solid unragged line.

decrease in height during the control phase of the $30 \mathrm{~h}$ experiment (Figure $4.3 \mathrm{~b}$ ) is characteristic of those experiments. A perfect equilibrium (horizontal tangent) during the compression phase of the $30 \mathrm{~h}$ experiments was only reached in two cases.

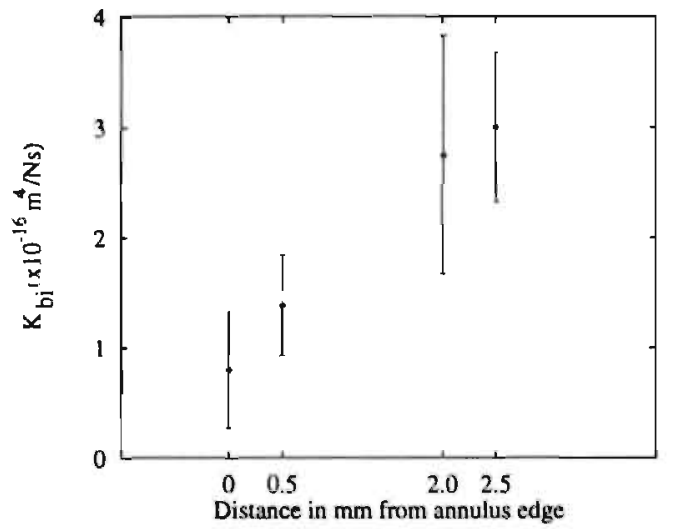

Figure 4.4: Average biphasic permeability coefficients \pm SD (error-bars) for the groups osi at $0.0 \mathrm{~mm}$, osf at 0.5 $\mathrm{mm}$, isi at $2.0 \mathrm{~mm}$, isf at $2.5 \mathrm{~mm}$ from the outer edge of the annulus (distances are average distances). distance from the annulus edge is scaled onto the horizontal axis of the plot, beginning with zero distance at osi. For all matching pairs of inner/outer samples of which both the permeability coefficients had been measured successfully ( 3 isf, 3 osf, 8 isi, and 8 osi), the permeability coefficient of the inner sample was always higher. isf) we calculated the average permeability coefficients and standard deviations. We did this also for ositosf (outer samples) and isitisf (inner samples). In Figure 4.4 the results are given for the first 4 groups. For the outer $(0.0-0.5 \mathrm{~mm}$ from annulus edge) and inner samples (2.0-2.5 mm from annulus edge) we found for $K_{b i}$ : outer: $K_{b i}=(1.02 \pm 0.57)^{*} 10^{-16}$ $\mathrm{m}^{4} / \mathrm{Ns}$,

inner: $K_{b i}=(2.81 \pm 0.98)^{*} 10^{-16}$ $\mathrm{m}^{4} / \mathrm{Ns}$.

In Figure 4.4 a clear rising of the permeability coefficients toward the inner regions of the annulus can be seen. The results have been displayed in such a way that the average

For each of the 4 groups (osi, isi, osf, 


\begin{tabular}{|c|c|c|c|c|c|}
\hline & osi & osf & isi & isf & isi+isf \\
\hline osi & & 0.0027 & $<0.0001$ & 0.0013 & \\
osf & & & 0.0011 & 0.0046 & \\
isi & & & & $0.3462^{*}$ & \\
osi+osf & & & & & $<0.0001$ \\
\hline
\end{tabular}

Table 4.1: One tailed $\mathrm{P}$ values (Mann-Whitney $U$ test) for differences in permeability coefficients. The bypothesis is tested that the permeability coefficients of the vertical groups are smaller than those of the horizontal groups. The only non significant $P$ value on a $5 \%$ level is indicated with a $" * n$.
Differences in permeability coefficients were significant for all but 2 groups (isi and isf). In table 4.1 the 1-tailed probabilities are summarized, where the hypothesis had been tested that the radial permeability coefficient decreases in the outer direction.

\begin{tabular}{|c|c|c|}
\hline \multicolumn{3}{|c|}{ Regions } \\
\hline group & $K_{b i}\left({ }^{*} 10^{-16} \mathrm{~m}^{4} / \mathrm{Ns}\right)$ & $\mathrm{n}$ \\
\hline osi, D & $0.91 \pm 0.35$ & 6 \\
osi, V+VL & $0.73 \pm 0.63$ & 9 \\
isi+isf, D & $3.19 \pm 1.10$ & 4 \\
isi+isf, V+VL & $2.46 \pm 0.93$ & 6 \\
\hline \multicolumn{3}{|c|}{ Levels } \\
\hline osi, L7-S1 & $0.86 \pm 0.26$ & 6 \\
osi, L5-6 & $0.73 \pm 0.96$ & 4 \\
\hline
\end{tabular}

Table 4.2: A verage biphasic permeability coeffirients \pm SD of groups per region ( $D=$ dorsal, $V=$ ventral, $\mathrm{VL}=$ ventrolateral), and for levels L7-SI, L5-6 for osi samples.

are summarized.
We also compared permeability coefficients of dorsal versus ventral + ventrolateral regions for the osi samples and the inner (isi+isf) samples. Neither for the osi, nor for the inner samples was there a significant difference in permeability coefficients ( $P=0.22$ for osi samples, $P=0.48$ for isitisf samples; 2-tailed), although there was a trend for the dorsal samples to have a higher permeability coefficient. For the osi samples we compared the L7-S1 permeability coefficients with the L.5-6 permeability coefficients (all regions), and did not find a significant difference ( $P=0.26 ; 2$-tailed). In table 4.2 the results of region and level

An interesting relationship between the strain of the conditioning phase, $\epsilon_{c o n d}$, and the permeability coefficient was found. The strain of the conditioning phase was defined as the height difference between the conditioning plateau, and the maximum or minimum height during the beginning of the conditioning phase, divided by the height of the conditioning plateau. In the case of a maximum height, there was height loss during this phase, and a negative conditioning strain. In some cases there was only an estimate available of the maximum or minimum height, because data acquisition had begun after the sample had expanded in the measurement chamber, and begun to swell or be compressed. Therefore, the absolute values of the strains are in some cases underestimations. The conditioning strains of outer $(0.038 \pm 0.093)$ versus inner $(0.182 \pm 0.055)$ samples were significantly 


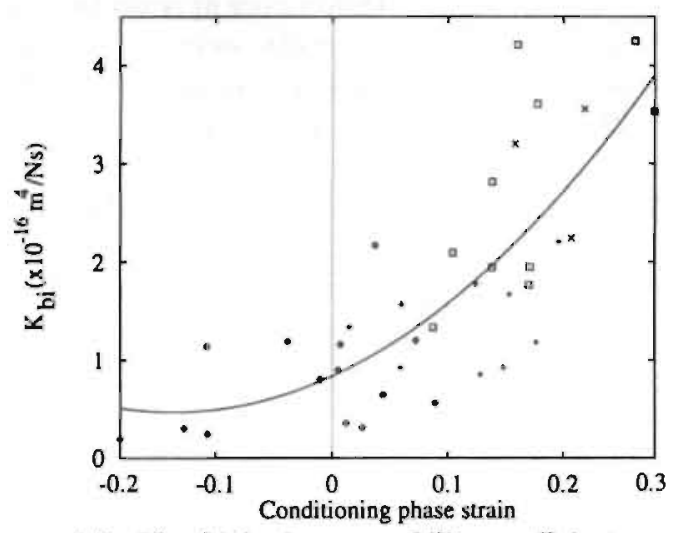

Figure 4.5: The biphasic permeability coefficient as a function of the strain of the conditioning phase, $\epsilon_{\text {cond }} .0$ : osi $_{i}+$ : osf, $\square$ : isi, $x$ : isf. Absolute values of strains are in some cases underestimated. The quadratic least squares regriession line is also given: $K_{b i}=\left(0.84+5.43^{*} \epsilon_{\text {cond }}+\right.$ $\left.19.92 * \epsilon_{\text {cond }}^{2}\right)^{*} 10^{-16} \mathrm{~m}^{4} / \mathrm{Ns}\left(R^{2}=0.61\right)$. smaller $(\mathrm{P}<0.00005)$. The permeability coefficient was found to rise with the conditioning phase strain as can be seen in Figure 4.5. Quadratic least squares regression gave an $R^{2}$ of 0.61 .

To test the relative importance of variation of $K$ with distance $d$ of the mid-sample to the annulus edge and conditioning phase strain $\epsilon_{\text {cond }}$, we performed a bilinear regression test on the variation of $K$ with $d$ and $\epsilon_{\text {cond }}$. as independent variables. We found that $K$ increased significantly with both $d$ and $\epsilon_{\text {cond }}$. The bilinear equation was $K=(0.538 * d+0.545 *$ $\left.\epsilon_{\text {cond }}+0.247\right)^{*} 10^{-16} \mathrm{~m}^{4} / \mathrm{Ns}$, where the distance was $d$ was measured in $\mathrm{mm}$. The $\mathrm{P}$ values were: $\mathrm{P}=0.0022$ for $d$ and $\mathrm{P}=0.0030$ for $\epsilon_{\text {cond }}$.

The average values of the compression phase strain $\epsilon$ and aggregate modulus $H_{A}$ (at 0.2 $\mathrm{MPa}$ ) for all the experiments were: $\epsilon=0.139 \pm 0.038, H_{A}=1.47 \pm 0.41 \mathrm{MPa}(\mathrm{n}=37)$, for the outer samples: $\varepsilon=0.127 \pm 0.023, H_{A}=1.56 \pm 0.34 \mathrm{MPa}(\mathrm{n}=24)$, and for the inner samples: $\epsilon=0.162 \pm 0.048, H_{A}=1.31 \pm 0.47 \mathrm{MPa}(\mathrm{n}=13)$. The values of $H_{A}$ of outer versus inner samples were significantly different at a $5 \%$ level $(2$-tailed exact $\mathrm{P}=$ 0.0148 ). This means that the outer samples were stiffer than the inner samples.

To assess if the PG loss resulted in a significant influence on the calculated results of $K_{b i}$ and $H_{A}$, we corrected for the PG loss, ${ }^{b}$ and again determined the $K_{b i}$ and $H_{A}$ values. The correction was done by assuming that before $5 \mathrm{~h}$ of testing no PG loss occurred ( 4.5 $\mathrm{h}$ for three experiments with a $4.5 \mathrm{~h}$ conditioning phase), and after that a linear decrease in sample height was assumed. The linear loss was defined by the line connecting the sample height at $5 \mathrm{~h}(4.5 \mathrm{~h})$, and at the end of the experiment $(15 \mathrm{~h}$, or $30 \mathrm{~h})$. The 5 $h$ point was taken because it marks the end of the conditioning phase for all but three $15 \mathrm{~h}$ experiments, and it is close to the $5.56 \mathrm{~h}$ found by Best et al. within which time no PG loss was found to occur. For the $30 \mathrm{~h}$ experiments the conditioning phase was almost in equilibrium after $5 \mathrm{~h}$, so the height at $5 \mathrm{~h}$ was also a close approximation of the equilibrium height for those experiments.

\footnotetext{
${ }^{\mathrm{b}} \mathrm{PG}$ loss was not directly measured, but inferred from height loss in the time-(sample height) curve.
} 


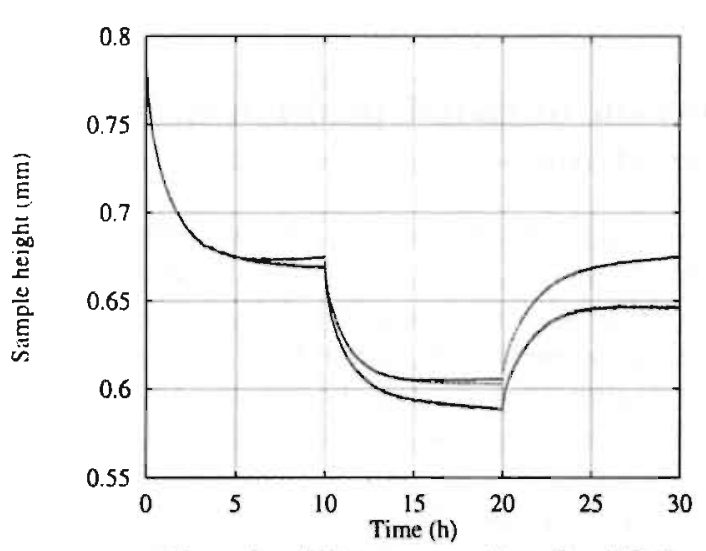

Figure 4.6: Example of linear correction for $P G$ loss. From $5 \mathrm{~h}$ on, the linear assumed loss is added to the sample height. This results in the upper curve. The lower curve gives the original data. The dashed line (visible in the compression phase under the top curve) is the biphasic fit.
An example of a PG correction is given in Figure 4.6. The top curve is corrected for PG loss, the bottom curve the original one. The dashed line, which is just visible in the compression phase under the top curve, is the fit of the PG corrected curve. For this correction the $K_{b i, c o r r}$ was $0.204^{*} 10^{-16} \mathrm{~m}^{4} / \mathrm{Ns}$, with an uncorrected $K_{b i}$ of $0.195^{*} 10^{-16} \mathrm{~m}^{4} / \mathrm{Ns}$. The aggregate moduli were: $H_{A_{\text {rcorr }}}$ $=1.83 \mathrm{MPa}$, and $H_{A}=1.67 \mathrm{MPa}$.

After correction, the calculated values of $K_{b i}$ and $H_{A}$ were found to lie close to the uncorrected results. The average values were (the subscript "corr" indicates corrected results):

outer samples $(\mathrm{n}=24): K_{b i}=(1.02$ $\pm 0.57)^{*} 10^{-16} \mathrm{~m}^{4} / \mathrm{Ns}, K_{\text {bi,corr }}=(1.04 \pm 0.53)^{*} 10^{-16} \mathrm{~m}^{4} / \mathrm{Ns}(\mathrm{P}=0.0008) ; H_{A}=1.56 \pm$ : $0.34 \mathrm{MPa}, H_{A, \text { carr }}=1.67 \pm 0.36 \mathrm{MPa}(\mathrm{P}=0.23)$, inner samples: $(\mathrm{n}=13) K_{b i}=(2.81 \pm 0.98)^{*} 10^{-16} \mathrm{~m}^{4} / \mathrm{Ns}, K_{b i, c o r r}=(3.19 \pm 1.13)^{*} 10^{-16}$ $\mathrm{m}^{4} / \mathrm{Ns}(\mathrm{P}=0.0015) ; H_{A}=1.31 \pm 0.47 \mathrm{MPa}, H_{A, \text { corr }}=1.42 \pm 0.50 \mathrm{MPa}(\mathrm{P}=0.38)$.

The changes in the calculated values of $K_{b i}$ and $H_{A}$ for uncorrected and PG loss corrected data analysis were significant for the $K_{b i}$, and insignificant for $H_{A}$ (Wilcoxon statistic; $\mathrm{P}$ indicates the probability that the samples of the 2 groups come from the same population). The differences for $K_{b i}$ were significant in a Wilcoxon test, which is a matched pairs test. This means that after correction for PG loss the $K_{b i}$ value is expected to be higher. However, the differences above indicate that the inciease in $\boldsymbol{K}_{b i}$, especially for outer samples, is small. We further notice that the difference in $K_{b_{i}}$ of outer versus inner samples is enhanced after PG correction. 


\subsection{Discussion}

\subsubsection{Permeability as a function of radial position in the disc, and conditioning phase strain}

From the results in Figure 4.4 it is clear that, derived on the basis of linear biphasic theory, the radial permeability coefficient of annulus fibrosus decreases towards the edge.

Conditioning strain has been found to correlate with the permeability coefficient. The smaller the strain, the smaller the permeability coefficient (Figure 4.5). The conditioning strain for outer samples was significantly smaller (in a number of cases negative, implying a shrinkage of the specimen in the conditioning phase) than that of the inner samples. This means that the outer samples, physiologically subjected to low compressive loads, ${ }^{138}$ tend to shrink or swell a little in the conditioning phase, whereas the inner samples, physjologically subjected to higher compressive loads, tend to swell more in the conditioning phase. Because the inner samples swell more during conditioning than outer samples, inner samples are expected to have a higher permeability coefficient after conditioning. It is thus the difference in conditioning swelling behaviour, that can at least in part explain the differences found in the biphasic permeability coefficients of outer versus inner samples. The results from the bilinear regression test verify this, but do at the same time demonstrate that the increase of $K$ with $d$ can not be entirely explained by the increase of $K$ with $\epsilon_{\text {cond }}$.

The edge of the annulus is covered by a small layer of semi translucent material of thickness about $0.5 \mathrm{~mm}$. It can be discerned from the adjacent part, which is opaque and milky white. Because the osf samples had been flattened on the turning lathe, part or all of the outer semi translucent layer had been removed, leaving less of this material compared to the osi samples. Also, the osf samples were uniform in thickness in contrast to the osi samples. The inner samples contained only opaque material. It is the authors' opinion, that the outer semi translucent layer is in part responsible for the decrease in the radial permeability coefficient of annulus samples toward the edge.

Urban and Maroudas ${ }^{22}$ found the permeability coefficient of human discs to increase with increasing hydration. This is in accordance with our finding of increasing $K_{\text {ti }}$ when going from outward to inward regions in the annulus, because hydration increases in that direction in the annulus. ${ }^{219}$

Drost et al. ${ }^{51}$ did not test radial dependency of the permeability coefficient, but compared radial (with the specimen's surface parallel to the axis of the spine) with axial specimens (surface perpendicular to the axis of the spine). They used the same experimental techniques and data processing as we did. They found $K_{b i}$ to be $(1.8 \pm 0.5)^{*} 10^{-16}$ $\mathrm{m}^{4} / \mathrm{Ns}$ for radial specimens, and $(3.2 \pm 1.5)^{*} 10^{-16} \mathrm{~m}^{4} / \mathrm{Ns}$ for axial specimens. Their radial value corresponds well with our (radial) value: $(1.6 \pm 1.1)^{*} 10^{-16} \mathrm{~m}^{4} / \mathrm{Ns}$.

Best et al. ${ }^{17}$ found values for the radial permeability coefficient $K_{b i}$ (human L3-4, L4-5 discs) corresponding in order of magnitude to our canine values (we give the overa!l average): $(2.5 \pm 1.1)^{*} 10^{-16} \mathrm{~m}^{4} / \mathrm{Ns}$. They found no dependence on position whatsoever (radial position, level of the disc, or region in the disc). They also used a confined compression setup and biphasic theory. However, they did not apply the same mechanical load for each experiment, but selected stresses "based on preliminary tests, to induce physiological levels of strain $(6-10 \%)$ in the tissue, and to ensure that in vitro strains 
did not exceed the assumptions of the linear model used to describe the mechanical behaviour of the annulus fibrosus". Their compressive stresses were in the range of 0.02 to $0.11 \mathrm{MPa}$. We used the same compressive stress of $0.194 \mathrm{MPa}$ for each experiment. The chemical loads were comparable: $0.15 \mathrm{M}$ for Best et al. and $0.16 \pm 0.01 \mathrm{M}$ for our experiments.

To explain the difference in our average overall value of the permeability coefficient and that of Best et al, it is probable that the difference in applied mechanical loads is responsible. We used a higher load than Best et al., which results in a lower biphasic permeability coefficient. As to the fact that Best et al. did not find significant differences of $K_{b i}$ between outer and inner samples, and we did, there are a number of possibilities. The use of different protocols (constant strain versus constant stress), is one probable cause. It is also likely that Best et al. did not perform experiments with a certain amount of semi translucent material, which in our opinion can be responsible for the lowering of $K_{b i}$ of outer samples. Finally, we performed experiments on canine discs, and they used human material.

Best et al. used shorter total experimentation times (less than $4.17 \mathrm{~h}$ ), because they could operate their loading mechanism in displacement control mode. In this fixed displacement mode it appeared that samples were equilibrated faster (less than $1.39 \mathrm{~h}$ ). Although we used longer times $(15 \mathrm{~h}$ ) for permeability coefficient determination, we do not believe that this influenced the differences found in permeability coefficients of outer and inuer samples. We found that the effects of PG loss on the calculated coefficients is small (see the section on leaching of $\mathrm{PG}$ ).

Wo found no evidence for a dependence of the permeability coefficient on level in the spine (L7-S1 versus L5-6), or region in the spine (dorsal versus ventral + ventrolateral). It was not the aim of this paper to test differences of material properties of IVD annulus along the complete spine. Our finding of a non-dependence of $K_{b i}$ on the levels L7-S1 versus L5- 6 does therefore not mean that there can not be a dependence when different levels are chosen.

\subsubsection{Approximation of the permeability coefficient}

Determination of the permeability coefficient was done using linear biphasic theory, with the equations given by Terzaghi. ${ }^{211}$ It implies assumptions of linearity of the stress-strain relationship of the solid, the absence of a swelling pressure, and $K_{b i}$ is assumed to be constant. Recently developed chemo-mechanical triphasic theories, ${ }^{109,200}$ and numerical implementations of those ${ }^{71,203}$ deal with the mechanics of swelling materials in a physically more realistic fashion, incorporating swelling pressure, deformation dependency of permeability and diffusion. Usage of triphasic models to compute permeability coefficients by fitting experimental data is hampered though by insufficient knowledge of a number of triphasic parameters and constitutive behaviour of cartilaginous materials, such as the stress-strain relationship for the solid, activity coefficients for water and ions, the osmotic coefficient, and the deformation and concentration dependency of permeability and diffusion. " Therefore we judged that it is beyond the scope of this study to fit triphasic simulations of compression experiments to their experimental counterparts.

\footnotetext{
"The stress-strain relationship for the solid, and the deformation dependency of the permeability are
} also unknowns in biphasic theory. 


\subsubsection{The aggregate modulus}

The aggregate modulus $H_{A}$ was found to differ significantly between outer and inner samples. The outer samples were stiffer. This is difficult to interpret physically because in biphasic theory solid stiffness and osmotic stiffness are lumped into one parameter. This means that the difference in total stiffness could be a result of differences in either of these stiffness parameters. Also, $H_{A}$ is deformation dependent, but was assumed constant. To compare $H_{A}$ with results from other researchers, the chemical equilibrium conditions (the external salt concentration), as well as the mechanical loading conditions need to be the same. This is neither the case for Best et al., ${ }^{17}$ nor for Drost et al. ${ }^{51}$

We applied a mechanical load of $0.194 \mathrm{MPa}$, and a chemical load of $(0.16 \pm 0.01)$ $\mathrm{M} \mathrm{NaCl}$, and found for the overall $H_{A}: H_{A}=(1.47 \pm 0.41) \mathrm{MPa}$. Best et al. applied mechanical loads ranging from 0.02 to $0.11 \mathrm{MPa}$, and a chemical load of $0.15 \mathrm{M} \mathrm{NaCl}$, and found: $H_{A}=(0.38 \pm 0.16) \mathrm{MPa}$. Drost et al. used $0.194 \mathrm{MPa}$, and $0.2 \mathrm{M}$ and found for the overall $H_{A}$ of the compression stage for radial samples: $H_{A}=(0.66 \pm 0.30) \mathrm{MPa}$. For axial samples they found $(1.01 \pm 0.31) \mathrm{MPa}$.

We found a significant difference in $H_{A}$ between outer and inner samples: outer samples: $H_{A}=(1.56 \pm 0.34) \mathrm{MPa}$, inner samples: $H_{A}=(1.31 \pm 0.47) \mathrm{MPa}$. The strains for outer and inner samples were: outer samples: (12.7 \pm 2.3$) \%$, inner samples: (16.2 \pm $4.8) \%$. Best et al. did not find significant differences between outer and inner samples, but did find a trend for middle and outer samples to be stiffer than innermost samples. They also did find a significant negative correlation between $H_{A}$ and the water content. As water content decreases from inner to outer annulus, this corresponds with the finding of a stiffer outer annulus.

Thus, although the methodology of Best et al. did not lead to the conclusion of differences in permeability coefficient of inner versus outer annulus, they did find a trend for the outer annulus to be stiffer. We found both for the permeability coefficient and the aggregate modulus a significant difference between outer and inner annulus samples: from inner to outer annulus the permeability decreases, and the aggregate modulus increases.

\subsubsection{In vitro versus in vivo state}

The values for the mechanical and chemical loads on the samples were chosen within the range of physiological loads for humans. The salt concentration $(0.16 \pm 0.01 \mathrm{M})$ is physiological. The mechanical loads of $0.0772,0.0778$, and $0.194 \mathrm{MPa}$ compare with in vivo values found in the literature. ${ }^{9,151}$ For the supine position the average load on a human L3-4 disc is about $0.11 \mathrm{MPa}$. Keller et al. ${ }^{98}$ found for the disc pressure in the central part of the nucleus in the normal immature porcine disc $0.0357 \pm 0.0060 \mathrm{MPa}$ (L1-2 disc, prone position, general anaesthesia).

Lacking data on the physiological range of disc pressures, and salt concentrations in the canine, we have taken recourse to human data presented above, and to one measurement in the porcine disc. In a study of Zimmerman et al. ${ }^{239}$ the canine disc showed a similar axial stiffness (after normalizing for size differences), but significantly higher torsional modulus compared to human values. Differences of more than a factor 3 between the intradiscal pressures of humans and canines are unlikely, because of the comparable anatomy and axial stiffnesses of the discs and a difference in size of a factor 2 to 3 . 
Deep freezing and storage of samples of cartilaginous tissues may cause changes in the material properties of these tissues. A number of researchers have investigated the influence of deep freezing and storage on the mechanical properties of cartilaginous tissues. Rabbit annulus fibrosus samples were investigated by Hickey and Hukins. ${ }^{77}$ They found no significant changes in the distribution of collagen fibrils in the sample (by Xray measurement) for three methods of conservation: (1) fixation in formol saline, (2) freezing, (3) freezing in liquid nitrogen. Fixation, however, caused the collagen molecules to be more closely packed. Panjabi et al. ${ }^{170}$ investigated the response of human thoracic motion segments to compressive, torsional, and bending loads as a function of storage time. The specimens were stored in a deep freezer. The freezing procedure consisted of sealing the spinal segments in double plastic bags and storing at $-18^{\circ} \mathrm{C}$. The specimens were thawed at room temperature $\left(22^{\circ} \mathrm{C}\right)$ at the time of testing. They concluded that the biomechanical properties of the spinal specimens were not significantly altered by either short- or long-term storage (up to 7 months) in air-tight containers at $-18^{\circ} \mathrm{C}$. Kwan et al. ${ }^{107}$ tested the effect of storage (not deep frozen) on the biomechanical behaviour of articular cartilage for large strains. They stored medial plateaus of the right knee of mongrel canines in closed containers at $4^{\circ} \mathrm{C}$ filled with a culture media. The plateaus were stored for either $3,7,28$, or 60 days. They used confined compression tests to compare the mechanical behaviour. Their conclusion was that stored articular cartilage maintains a similar ability as normal fresh tissues in response to large applied stresses, and that the effects on storage time on the compressive modulus are insignificant. In our own laboratories (results not published) the viscosity of nucleus pulposus material was tested on a rheometer. The conclusion of these experiments was that deep freezing slightly alters the viscosity of the specimens.

The storage temperature for the experiments in this study was $-65^{\circ} \mathrm{C}$, and average time of storage $38.6 \pm 15.7$ days. On the basis of the facts presented above, we do not believe that there were gross changes in the mechanical properties of the annulus specimens we tested, and that the sample's state directly after thawing approximately represented the in vivo state.

The temperature at which we conducted the experiments $\left(22 \pm 1^{\circ} \mathrm{C}\right)$ was well below the physiological temperature $\left(37^{\circ} \mathrm{C}\right.$ ). This was done for practical purposes (lab temperature), and had the additional advantage that autolysis is reduced compared to the situation, where testing is done at physiological temperature. The influence of this temperature difference was not investigated.

\subsubsection{Leaching of proteoglycans}

Leaching of proteoglycans during testing influences the mechanical behaviour of the tissue. Periods of equilibration substantially longer than $18 \mathrm{~b}$ lead to losses of GAG (glycosaminoglycans) for cartilage. ${ }^{134}$ Best et al. ${ }^{17}$ found no changes in equilibrium isometric swelling pressure or aggregate modulus only within the first $5.56 \mathrm{~h}$ of testing (annulus of IVD). During our experiments the samples suffered material loss, especially for the $30 \mathrm{~h}$ experiments. Most of the 30 h experiment samples ( $84 \%$ ) showed a significant progressive decrease in height during the control stage, an example of which can be seen in the right graph of Figure 4.3. The height loss of outer versus that of inner samples at the end of the experiment $(15 \mathrm{~h}$ or $30 \mathrm{~h})$ was somewhat higher for inner samples $((5.7 \pm 2.1) \%$ versus 
$(3.8 \pm 3.1) \%$ ), but this difference was a lot smaller after $15 \mathrm{~h}$ (a time which was not exceeded for the determination of coefficients) for the $30 \mathrm{~h}$ experiments. The influence of correction for height loss turned out to be small for the permeability coefficient values, and the differences for $K$ between inner and outer samples were even enhanced. We can thus conclude that leaching of $\mathrm{PG}$ does not alter the result of this study that the $K$ of outer samples is significantly lower than that of inner samples.

\subsubsection{Conclusions}

The biphasic permeability coefficient decreases with decreasing distance to the annulus edge. Whether this finding explains the discrepancy between the model simulation and experiment with respect to bulging of the edge of the annulus, should be investigated further. Similar experimental studies on the permeability coefficient of the end plate could shed more light on the boundary conditions along this outflow surface. 
Chapter 5

\section{Triphasic material parameters of canine anulus fibrosus}




\subsection{Summary}

Study design. The chemo-mechanical material properties for swelling and compression of slices of annulus cut from canine intervertebral discs were determined, as well as the permeability coefficient and aggregate modulus, as a function of radial position in the disc. A triphasic one-dimensional computer model was assessed.

Objectives. The purpose was to determine material properties in radial direction as a function of radial position, distinguishing between elastic, osmotic, and viscous forces, and to test a computer implementation of the triphasic mixture model for intervertebral disc.

Summary of background data. The deformation of intervertebral disc tissue in response to external load is associated with (1) elastic forces, (2) osmotic forces, and (3) viscous forces. Mixture models have been designed to separate the influences of the constituents to the chemo-mechanical behaviour of swelling porous materials, such as cartilage and intervertebral disc.

Methods. Confined swelling and compression (CSC) experiments were designed to measure the time course of uni-axial deformation of samples of diameter $4 \mathrm{~mm}$, and height 1 mm. The rotation symmetry axis of the samples was taken in the radial direction of the disc. Chemo-mechanical material properties such as osmotic coefficients, activity coefficients, fraction of intrafibrillar water and an effective stress-strain law were determined from the time-displacement data, and using semi-empirical laws and literature data. The equilibrium results were interpreted in terms of Donnan osmotic theory, the division of the fluid compartment into an extra- and intrafibrillar compartment, and porous media concepts. The time-displacement data including transients were fitted with computer simulations of a one-dimensional implementation of the triphasic model, based on one fluid compartment. Fixed charge density and hydroxyproline measurements were performed as necessary input for the model equations. Linear biphasic theory was used to determine biphasic radial permeability coefficients and aggregate moduli. Approximations of in vivo triphasic permeability coefficients were determined from the computer fits of the $\mathrm{CSC}$ experiments.

Results. The fixed charge density, bi- and triphasic permeability coefficients and aggregate modulus increased with distance from the outer annulus, hydroxyproline decreased. (A) equilibrium 2-fluid compartment results. The material appeared to be under compression in radial direction in the in vivo situation, as well as in most cases of the experimental equilibrium states. A linear fit between the (2nd Piola-Kirchhoff) effective stress and (Green-Lagrange) strain yielded for the effective stiffness: $H_{\epsilon}=1.09 \pm 0.66$ $\operatorname{MPa}(n=20)$. The average fraction of intrafibrillar water was $1.16 \mathrm{~g} / \mathrm{g}$ collagen. The results were sensitive for changes in the activity and osmotic coefficients, and the fraction of intrafibrillar water.

(B) computer fit with one fluid compartment. Triphasic computer fits based on a single compartment (no distinction between intra- and extrafibrillar fluid) yielded parameter values close to the two-fluid-compartments equilibrium results. The equilibria and compression transient could easily be fitted using the two-fluid-compartments parameter values as starting input. The swelling transient, however, could not be fitted concurrently with the compression transient.

Conclusions. This study demonstrates how confined swelling and compression experi- 
ments can be combined with a number of physico-chemical measurements to separate the elastic, osmotic, and viscous contributions of annulus fibrosus to the overall behaviour of the intervertebral disc. The triphasic (one-fluid-compartment) model can be used to simulate the CSC experiment with parameter values that are close to the two-fluidcompartments equilibrium analysis results. Biphasic and triphasic vivo permeability coefficients were shown to be greater for inner annulus compared to outer annulus. [Key words: annulus fibrosus, intervertebral disc, triphasic, mechanics, permeability, aggregate modulus, biphasic, canine]

An abridged version of this chapter was submitted to Spine with co-authors: Jarques M. Huyghe, Maarten R. Drost, Jan D. Janssen, and Anthony Husun. 


\subsection{Introduction}

Because of the role the intervertebral disc (IVD) annulus fibrosus (AF) may play in the etiology of low back pain, e.g. in disc herniations, ${ }^{39,43,78,90,165}$ it is important to understand its mechanical functioning. The annulus contains about $70 \%$ of water (by wet weight), $10-20 \%$ proteoglycans (PG) by dry weight, and $67 \%$ of collagen by dry weight. ${ }^{54}$ The AF is organized into circumferential fibrous lamellae that connect to the nucleus, end plates, vertebral bodies, and ligaments. There is a steady increase in the proportion of collagen and a decrease of PG content from the inner to outer annulus, ${ }^{5,54,118}$ although for collagen content this has not always been confirmed. ${ }^{199}$

AF has a strong swelling propensity because of the presence of the hydrophilic PG. The fixed negative charges on these $\mathrm{PG}$ are the main cause of tissue swelling. This swelling behaviour can be explained in terms of Donnan osmotic pressure, ${ }^{134,219,222,224,225}$ charge to charge repulsion forces (chemical expansion), ${ }^{5,53}$ or both. ${ }^{109}$ The swelling tendency increases with the concentration of the fixed negative charges, which is called the fixed charge density (FCD).

Urban and McMullin, ${ }^{224,225}$ Maroudas and Bannon, ${ }^{130}$ and Maroudas and colleagues $93,135,232$ have shown that the concentration of fixed charges inside the tissue should be based on the amounts of extrafibrillar water. In cartilaginous tissues part of the water is trapped inside the collagen fibrils. This fraction of the total tissue water is called the intrafibrillar water. The PG, because of their large size, are excluded from this intrafibrillar space. Maroudas et al. ${ }^{135}$ performed experiments to determine the dependence of the fraction of intrafibrillar water on the pressure. They loaded proteoglycan-free bovine articular cartilage with either a mechanical (unconfined compression) or an osmotic pressure (sample in dialysis sac, immersed in $0.15 \mathrm{M} \mathrm{NaCl}+\mathrm{P}$ (oly)E(thylene) G(lycol) solution), and found that for both types of loading the pressure-total water relationship is the same. Thus the amount of extra- + intrafibrillar water for PG depleted bovine cartilage is the same function for mechanical and osmotic pressures. To determine the amount of intrafibrillar water as a function of pressure they used an X-ray scattering technique. The diffraction patterns of the X-rays yield information on the lateral packing of collagen molecules. From the interaxial spacing of the collagen molecules, the mass of intrafibrillar water per $\mathrm{g}$ of collagen, $\phi_{c i}$, can be calculated.

Maroudas and colleagues also tested the dependence of the intrafibrillar water content of native cartilage as a function of PEG osmotic pressure. They found that the PEG osmotic pressure- $\phi_{c i}$ profile was the same for native and depleted cartilage. Thus, the intercollagen spacing is the same whether the osmotic pressure on the fibres comes directly from PEG (PG depleted cartilage), or from the PG from the extrafibrillar space, which, in their turn were compressed by the PEG across a dialysis membrane (native cartilage). From this they concluded that the intercollagen spacing and thus the intrafibrillar water content in cartilage is regulated primarily by the magnitude of the osmotic pressure gradient between the extra- and intrafibrillar compartments. ${ }^{93,135,231}$

For IVD no studies have been performed to determine $\phi_{c i}$ under well defined loading conditions. Urban and $\mathrm{McMullin}^{224}$ determined a value of 1.33 for $\phi_{c i}$ by comparing osmotic pressures of extracted PG with swelling pressures of disc slices, assuming that $\phi_{c i}$ was constant. However, having fitted their data with a straight line to obtain the value for $\phi_{c i}$, they found that a quadratic relationship gave a better fit. Furthermore, 
they had not matched the extracted PG and disc PG for GAG composition.

Because of the PG exclusion from intrafibrillar water, the effective concentration of PG, the effective FCD, is higher than the value based on total tissue water. The osmotic pressure, estimated from extrafibrillar or effective FCD is higher than that based on total tissue water, and corresponds well with osmotic pressures determined from isolated PG solutions, both for cartilage, ${ }^{130}$ and IVD. ${ }^{224}$

Theories that describe the mechanical behaviour of cartilaginous tissues such as intervertebral disc annulus fibrosus can be divided into three categories. (1) The physicochemical theories that are based on microscopic or macroscopic continuum electrical models. The classical Donnan theory for aqueous polyelectrolyte solutions is an example of the macroscopic continuum electrical model. Physico-chemical theories do not explicitly model the solid phase. ${ }^{125,126,128,132,222}$ (2) Solid (single phase), ${ }^{60,73}$ or solid-fluid (biphasic) $)^{80,108,110,120,144,146}$ continuum theories that do not explicitly model PG electrical charge related swelling phenomena (ionic effects). (3) Models that take both ionic effects and solid deformation into account. They model the ionic effects either implicitly (hybrid biphasic models), ${ }^{52,53,112,113,147,150,193}$ or explicitly (triphasic models). ${ }^{109,200}$

Macroscopic physico-chemical models can be used to explain fluid and solute transport in soft hydrated tissues, ${ }^{125-128,132,215,217,218,222}$ and small ions distribution and swelling of these tissues. ${ }^{130,134,136,220,222-225}$ As these models do not explicitly take the solid phase into account, they are not able to account for viscoelastic behaviour of the tissue, which is in part due to intrinsic viscoclasticity of the solid. ${ }^{27,97-99,102,159,168,169,208,239}$ Neither can they be used for the modeling of failure mechanisms of the solid.

Biphasic theories have been reasonably successful in describing compressive behaviour of cartilaginous tissues, treating the collagen-proteoglycan matrix as a solid with the viscoelastic properties depending on all the underlying physico-chemical effects. However, biphasic theories are not equipped to model phenomena such as Donnan osmotic and chemical expansion swelling. These PG charge related phenomena are responsible for the pre-stressed state of the collagen fibres (collagen pre-stress, or elastic stress ${ }^{69,127,128,130}$ ), and increased fluid pressure, inside a tissue sample bathed in a solution of $\mathrm{NaCl}$, when there is no external mechanical load.

The first theory to model the ionic effect explicitly was reported by Lai et al. ${ }^{109}$ Their theory incorporates the Donnan ion distribution and osmotic pressure theory for polyelectrolyte solutions. They define three phases: (1) the interstitial fluid phase and (2) the collagen-proteoglycan solid phase, both immiscible, and a fluid miscible phase: (3) the ionic phase. In this triphasic theory, the stresses in the solid matrix and the chemical potentials for the interstitial fluid and ions are related to the Helmholz energy functions in accordance with the laws of energy balance and entropy. The triphasic theory thus unites the continuum biphasic theories, ${ }^{145,146}$ and macrocontinuum physicochemical theories ${ }^{50,127,128,219,220}$ for the mechanics of cartilaginous tissues. The theory does not distinguish between intra- and extrafibrillar water. According to Lai et al. ${ }^{109}$ the "one-fluid-compartment" Donnan osmotic pressure alone is not sufficient to account for the tissue swelling, and they introduced the chemical expansion stress (electrostatic repulsive forces exerted on the $\mathrm{PG}$-collagen network) to also contribute to the swelling behaviour of cartilage and to its mechanical stiffness in compression.

Snijders ${ }^{200}$ developed a similar triphasic theory, based on the theory of Lai et al.. The main difference between the two theories is that Snijders does not define chemical 
expansion stress, but only uses Donnan theory to account for the swelling. Snijders et al. ${ }^{203}$ also developed a numerical finite element (FE) model based on the triphasic theory with coupled differential equations. They demonstrated the ability of the model (1) to fit confined swelling and compression measurements of annulus fibrosus tissue ${ }^{202}$ and (2) to simulate compression of an axisymmetric intervertebral disc. ${ }^{201}$

To determine the triphasic material parameter values as input for the FE model the confined swelling and compression (CSC) experiment is very suitable. In the experiment a cylindrical sample of annulus material is confined in a cylindrical chamber. Compressive load is conferred to the sample by means of a loading piston that fits tightly in the chamber. The sample is placed in contact with a bathing solution of known osmotic strength by placing it on a filter through which the solution circulates (chemical load). The sample's height variation (piston displacement) in time as a function of mechanical and chemical load is measured. Because of the well defined sample geometry, and mechanical and chemical loads, the CSC experiment is a good tool to determine triphasic material properties, and also serves as a assessment instrument for the triphasic model.

In this study, the AF of the canine IVD was used as an animal model for the human AF. The goal of this study was to analyze experimentally the chemo-mechanical behaviour of AF. Unlike Lai et al., ${ }^{109}$ and Snijders et al., ${ }^{203}$ the analysis was not done on the basis of three phases (solid-water-ions), but on a more advanced approach, distinguishing between intra- and extrafibrillar compartments, the existence of which has been clearly demonstrated by Maroudas and colleagues, ${ }^{93,130,135,232}$ and Urban and McMullin. ${ }^{224,225}$ The swelling and compression phases were fitted with simulations from the one-dimensional computer program of chapter 3 . These fit results were compared with the two-fluid-compartments analysis of the equilibria of the CSC experiments. As a secondary goal the biphasic and triphasic permeability coefficients, and aggregate moduli were determined as a function of radial position.

From the experiments, determinations were made of the following chemo-mechanical properties of the AF: the ions average activity coefficient, the Donnan osmotic coefficicnt, the fraction of intrafibrillar water, the stress free deformation state, and an effective stressstrain relationship as a function of the radial position in the disc.

When the effective stress-strain relationship is estimated from a CSC experiment, the stress free length of the sample needs to be fitted at the same time. The stress free state of a cartilaginous tissue sample represents the state when the Donnan osmotic pressure, the fluid pressure and the mechanical load equal zero. The solid is then unstressed. It is difficult to attain this state experimentally, because in order to reach that state all the water would have to be extracted from the tissue. Theoretically, however, the stress free dimensions of a sample have to be known in a mixture model in order to determine a constitutive model for the effective stress versus strain.

In order to determine the mechanical properties, fixed charge density was measured using the tracer cation method, ${ }^{133}$ and collagen contents was estimated from the hydroxyproline content. ${ }^{6,228}$ Semi-empirical laws and literature data have been incorporated to calculate the ionic activity coefficients, ${ }^{106,121,233}$ osmotic coefficients, and fraction of intrafibrillar water. 


\subsection{Methods}

\subsubsection{Sample preparation}

The lower lumbar spines of 3 German shepherds ( 2 males, 1 female) were harvested postmortern. Body weights were 25, 28, and $40 \mathrm{~kg}$. Ages at death ranged from 1 to 3 years. Within 1-3 h after death the spines were dissected. Spines were sawed off above the lumbar region, and below L7-S1. The ventral sides of the discs were partially freed of muscle tissue, and of the longitudinal anterior ligament, leaving the surface of the discs intact for measurements. After dissection, the spines were sealed in plastic bags, and kept frozen at $-65^{\circ} \mathrm{C}$.

Within two weeks, the frozen spines were sawed, excising in radial direction parts of the discs in more or less rectangular slabs, of dimensions of roughly 15 (radial) $* 8$ (circumferential) ${ }^{*} 8$ (axial) $\mathrm{mm}^{3}$. The slabs were from discs L7-S1 to L2-3, and from either of the ventral (V), ventrolateral (VL), or dorsal (D) regions (Figure 4.1). The length axes of the slabs were approximately perpendicular to the outer surface of the annulus.

Directly after the slabs were sawed, they were glued into perspex cylindrical holders of inner diameter $10.5 \mathrm{~mm}$, or $11.7 \mathrm{~mm}$, with outer diameter $15.0 \mathrm{~mm}$, and height of $25-30$ $\mathrm{mm}$, with a closed bottom. The slabs were glued with the outer surface of the annulus perpendicular to the axis of the holders, the surface sticking out about $1 \mathrm{~mm}$. Tissue-tek (OCT Compound 4583, Miles Diagnostics, Elkhart, IN, 46515 USA) was used to glue the slabs. During gluing, the slabs were kept frozen, using liquid nitrogen $\left(-196^{\circ} \mathrm{C}\right)$. Upon contact with the liquid nitrogen, the tissue-tek solidified.

In case the outer edge of the annulus was polluted with blood, the surface was scraped clean with a scalpel, taking care not to damage it. Remnants of the spinal cord of dorsal specimens that had not been broken off cleanly during sawing, were also removed with a scalpel.

Within 4 days the glued slabs were turned on a turning lathe. Radial samples of approximately $1 \mathrm{~mm}$ height with a $3.9 \mathrm{~mm}$ diameter were turned keeping the chisel and slabs cooled with liquid nitrogen. When possible 2 or even 3 neighbouring samples per slab, with an interspace (lost material from the thickness of the chisel) of $1.0 \mathrm{~mm}$ were produced. The outer samples were either with intact outer annulus surfaces, or these were flattened on the lathe. Loss of material for flattening the outside surface of outer samples amounted to approximately $0.2-1.0 \mathrm{~mm}$. The inner samples were flattened on both sides on the lathe.

Of each sample the approximate distance (frozen state) of the middle to the outer annulus edge was calculated, making it possible to infer quantities such as permeability, fixed charge density, and porosity as a function of the distance to the annulus edge. The determination of this distance was biased by measurement errors that in some cases could amount to $0.5 \mathrm{~mm}$.

The samples were put in aluminum cups and stored to a maximum of 21 days before being used in the experiment.

During all the stages of preparation the samples had been kept frozen, using liquid nitrogen as a coolant. 


\subsubsection{Confined swelling and compression (CSC) setup}

Three identical measurement setups were used in parallel. A description of the testing apparatus (Figure 4.2a) is given in Snijders, ${ }^{200}$ and Drost et al. ${ }^{51}$ The heart of the experimental setup for the confined swelling and compression measurements consisted of a cylindrical stainless steel chamber of diameter $4.030 \pm 0.004 \mathrm{~mm}$. The bottom of this chamber was formed by a sintered glass filter (pore sizes $16-40 \mu \mathrm{m}$, permeability coefficient $10^{-12} \mathrm{~m}^{4} / \mathrm{Ns}$ ). The top of the chamber was closed off by a piston (Figure $4.2 \mathrm{~b})$.

Mechanical loading of the samples was accomplished by a cantilever arm on which the loading piston was mounted. Weights were hung on this arm, the compressive load of which was transferred via the piston. Chemical loading was done by flowing a bathing solution of $\mathrm{NaCl}$ through the filter. The loading arm was connected to a DC operated linear variable displacement transducer (LVDT, Schaevitz) interfaced by a Labmaster 12 bit AD converter (Scientific Solutions Inc., Solon, Ohio, U.S.A.) to an IBM-AT. A vibrator was attached to the setup to overcome sticking of the sample and piston to the chamber wall. $0.5 \mathrm{~s}$ after each sample point, the setup was vibrated at $50 \mathrm{~Hz}$ during $2 \mathrm{~s}$. The data acquisition sampling frequency was $0.125 \mathrm{~Hz}$. To allow for free movernent of the piston the measurement chamber was placed on a film of silicon oil, and the circumference of the piston greased with vaseline.

\subsubsection{Experimental protocol}

Using a scalpel, the frozen sample was freed of irregularities that resulted from the turning. The sample was then placed in the chamber, and the piston placed on top. During the thawing the sample automatically expanded against the wall, filter and piston. Air was expelled from the setup by use of a vacuum pump, which was used as the salt solution circulation was started.

After the three samples had been placed in the cylinders, and the salt solution was circulating, data acquisition was started. Sample height was measured by the LVDT as a function of time. The temperature was kept at $22 \pm 1{ }^{\circ} \mathrm{C}$. The load scheme consisted of 4 stages: the conditioning, swelling, compression, and control stages. The duration of each stage was chosen sufficiently long in order to reach equilibrium (no change in sample height in time). The mechanical and chemical loads for all stages are tabulated in table 5.1.

\begin{tabular}{|c|c|c|c|c|}
\hline & conditioning & swelling & compression & control \\
\hline$\overline{\mathrm{W}}(\mathrm{MPa})$ & 0.0772 or 0.0778 & 0.0772 or 0.0778 & 0.194 & $\overline{0.0772}$ or 0.0778 \\
\hline $\mathrm{C}(\mathrm{mol} / \mathrm{l})$ & $0.469 \pm 0.013$ & $0.159 \pm 0.007$ & $0.159 \pm 0.007$ & $0.469 \pm 0.013$ \\
\hline duration & 5 or $6 \mathrm{~h}$ & 5 or $6 \mathrm{~h}$ & 5 or $6 \mathrm{~h}$ & 5 or $6 \mathrm{~h}$ \\
\hline
\end{tabular}

Table 5.1: The loading stages of the experimental protocol. $\mathrm{W}=$ mechanical load, $\mathrm{C}=$ chemical load. For the chemical load the average $\pm S D$ for all the experiments $(n=23)$ is given. Per experiment more exact values were used.

The bathing solution was either (average of all experiments \pm SD) $(0.159 \pm 0.007) \mathrm{M}$ 
or $(0.469 \pm 0.013) \mathrm{M}$. Exact values per experiment were determined from concentration measurements. To keep the $\mathrm{pH}$ at a sufficiently high constant level the solutions were buffered with $5 \mathrm{mM}$ Tris, $\mathrm{pH}$ 7.9. Computer driven electromagnetic valves switched the circulation from one bathing solution to the other. The changes of weights were done using a PC driven step motor. The total testing time was either $20 \mathrm{~h}$ or $24 \mathrm{~h}$, divided in equal parts per phase. For outermost samples with either intact outer surfaces or a few tenths of a mm taken off for flattening $24 \mathrm{~h}$ experiments were used, for inner samples 20 h. The longer equilibration times for the outermost samples motivates this difference in experiment times.

For a sample to be chosen for further determinations after the CSC experiment the sample itself had to be free of bone, or nucleus material. An experiment was successful, if (1) the four phases were well equilibrated, i.e. the mean absolute height change per $\mathrm{h}$ of the last hour of a plateau amounted to no more than $0.4 \%$ of the height at the end of the plateau, and (2) the height at the end of the control phase differed less than $5 \%$ of the conditioning height. From visual inspection of the time-displacement curves, these demands were regarded acceptable. The average percentage height loss \pm SD of the height from the end of the conditioning to the end of the control stage was $1.72 \pm$ $1.17 \%$ of the end height of the conditioning stage $(n=23)$. The average height change of the last hour in $\% / \mathrm{h}$ of the end height of the plateau was calculated for all stages: conditioning: $0.123 \pm 0.093 \% / \mathrm{h}$, swelling: $0.056 \pm 0.066 \% / \mathrm{h}$, compression: $0.106 \pm$ $0.079 \% / \mathrm{h}$, control: $0.049 \pm 0.050 \% / \mathrm{h}$.

The above criteria resulted in 23 successful experiments from a total of 79 . The 23 samples were distributed among the 3 dogs as follows: 8 from $\operatorname{dog} 1$ (male; $28 \mathrm{~kg}$ ), 2 from $\operatorname{dog} 2$ (female; $25 \mathrm{~kg}$ ), and 13 from dog 3 (male; $40 \mathrm{~kg}$ ).

\subsubsection{Data analyses}

Chemical analyses; dry weight, fluid volume, hydroxyproline, fixed charge density

Wet weight and height (volume), dry weight, hydroxyproline content, and the fixed negative charges (FC) of all samples were measured. From these quantities total water content and fraction, collagen content, intra- and extrafibrillar water content, density of the solid, and fixed charge density ( FCD; symbol: $c^{p g}$ ) were determined. At the end of the experiment the height of the piston was measured, the sample was taken out and blotted to remove adhering water. Directly after this the wet (total) weight was measured. The sample was stored frozen $\left(-65^{\circ} \mathrm{C}\right)$ before it was freeze dried $(24 \mathrm{~h})$ for dry weight determination.

Total water content and fluid fraction at sample height h were determined as follows:

$$
\begin{array}{r}
V^{t}(h)=h * A, \\
m_{e}^{J}=m_{e}^{t}-m^{t}, \\
V^{f}\left(h_{e}\right)=\frac{m_{e}^{f}}{\rho^{f}}, \\
V^{f}(h)=V^{f}\left(h_{e}\right)+\left(h-h_{e}\right) * A,
\end{array}
$$




$$
\phi^{f}=\phi^{f}(h)=\frac{V^{f}(h)}{V^{t}(h)} .
$$

with $V^{t}(h)$ the sample volume at height $h, A$ the area of the sample, $m_{e}^{f}$ the fluid mass at the end of the experiment, $m_{e}^{t}$ the total or wet mass at the end of the experiment, $m^{s}$ the solid or dry mass (after freeze drying), $V^{J}(h)$ the fluid volume at height $\mathrm{h}, h_{\varepsilon}$ the sample height at the end of the experiment, $\rho^{f}$ the density of the fluid, and $\phi^{\prime}(h)$ the fluid fraction at height $h$.

When the fluid fraction $\phi_{0}^{f}$ at height $h_{0}$ is known, the fluid fraction at height $\mathrm{h}$ can be calculated from:

$$
\phi^{f}=1-\frac{1-\phi_{0}^{f}}{J}
$$

with $J=h / h_{0}$ the elongation factor.

After the dry weight was measured, the sample was cut in two, and the dry weights of each half were measured. One half was used for hydroxyproline determination, the other half for FCD measurement.

To determine the collagen content, the \% hydroxyproline on dry weight basis (hyp dw) was measured using a colorimetric assay. ${ }^{207} \mathrm{~A}$ factor of 7.55 was employed to convert hydroxyproline to collagen. ${ }^{6,228}$

FCD was measiured with the tracer cation method, ${ }^{133,219}$ using ${ }^{22} \mathrm{Na}$ (Dupont de Nemours, Mechelen, Belgium) as tracer. The samples were equilibrated for at least 48 $\mathrm{h}$ in a $0.015 \mathrm{M} \mathrm{NaCl}, 25 \% 20,000$ polyethyleneglycol (PEG) solution, spiked with 0.1 $\mu \mathrm{Ci} / \mathrm{ml}^{22} \mathrm{NaCl}$. After dry weight measurement the samples were put in dialysis sacs (molecular weight cut-off 15,000; Spectrum, Laguna Hills, CA, 92653 USA) to prevent PEG from penetrating into the tissue. Equilibrating solutions were sealed off from contact with air to prevent discharge of carboxyl groups. Because of Donnan equilibrium the $c^{p s}$ approximately equals the $\mathrm{Na}^{+}$concentration, measured from the counts of the ${ }^{22} \mathrm{Na}^{219}$

FCD on dry weight basis, $c_{d w}^{p g}$, was calculated as follows. (1) A fraction of known weight was taken from the equilibrating solution. $1 \mathrm{~g}$ of this fraction contained $1.125^{*} 10^{-5} \mathrm{~mol}$ $\mathrm{NaCl}$, yielding $y$ counts per minute. $x \mathrm{cpm}$ from the tissue thus corresponded with $v=$ $x^{*}\left(1.125^{*} 10^{-5} / y\right)$ mol Na+. (2) The fixed charge density on dry weight basis, $\left(c_{d w}^{p g}\right)$, is now given by: $c_{d w}^{p g}=v /(d r y$ weight $)$.

\section{Equilibria}

During an experiment there were 4 equilibrium states: conditioning ("0"), swelling ("sw"), compression, and control. For the first. 3 equilibrium states we determined: (1) intra- and extrafibrillar water content, (2) the FC.D on extrafibrillar water basis, $c_{e x f}^{p g}$, (3) the second Piola-Kirchhoff effective stress as a function of linear strain.

Intra- and extrafibrillar water content. The extrafibrillar water volume, $V_{e x f}^{f}$, is the difference between the amount total water, $V^{\prime}$, and intrafibrillar water $V_{\text {inf }}^{f}$ :

$$
V_{e x f}^{f}=V^{\prime}-V_{\text {inf }}^{f}
$$


Intra- and extrafibrillar water content was determined from the collagen content. Collagen content (in \% of dry weight) was assumed equal to the hydroxyproline content multiplied by $7.55 .^{6,228}$ Intrafibrillar water mass is a factor $\phi_{c i}$ times the collagen mass $\left(\phi_{c i} \mathrm{~g}\right.$ water per $g$ of collagen). ${ }^{130,135,136,224,232}$ Maroudas et al. ${ }^{135}$ performed experiments to determine the dependence of $\phi_{c i}$ on the osmotic pressure difference between extra- and intrafibrillar compartments $\Delta \pi$ (in atm) for articular cartilage. We fitted (least squares) their $\phi_{c^{-}}$ pressure relationship derived from $\mathrm{X}$-ray data with an exponential function:

$$
\phi_{c i}=0.448 e^{-0.328 \Delta \pi}+0.822,
$$

Regarding the equilibrium relations for fluid flow between the external ("ext"), extrafibrillar ("exf") and intrafibrillar ("inf") compartments, we get:

$$
(p-\pi)_{\text {ext }}=(p-\pi)_{e x f}=(p-\pi)_{\text {inf }},
$$

where $p$ is the fluid pressure, and $\pi$ the osmotic pressure. The external and the intrafibrillar solutions only contain $\mathrm{NaCl}$. Equilibrium between the intra- and extrafibrillar compartments requires that the salt concentration in the intrafibrillar compartment is in Donnan equilibrium with the extrafibrillar compartment. Therefore, the intrafibrillar salt concentration equals the external salt concentration, and their osmotic pressures at equilibrium are the same:

$$
\pi_{\text {ext }}=\pi_{\text {inf }} .
$$

The osmotic pressure difference between the extrafibrillar and intrafibrillar compartments is given by:

$$
\Delta \pi=\pi_{e x f}-\pi_{i n f} .
$$

The boundary condition for momentum is given by:

$$
\boldsymbol{\sigma}_{e, e x t} \cdot \boldsymbol{n}-p_{e x t}=\boldsymbol{\sigma}_{e, e x f} \cdot \boldsymbol{n}-p_{e x f}=\boldsymbol{\sigma}_{e, i n f} \cdot \boldsymbol{n}-p_{i n f},
$$

where $\sigma_{e}$ is the effective Cauchy stress tensor, $\boldsymbol{n}$ a unit vector, and $p$ the fluid pressure. The external pressure can be chosen freely. For convenience a zero value is chosen. Then, if we choose $\boldsymbol{n}$ parallel to the loading direction, as in our experiment, the $\boldsymbol{\sigma}_{e, c x t} \cdot \boldsymbol{n}$ equals the minus of the applied mechanical pressure $-F / A$. A zero $p_{\text {ext }}$ together with eqs. (5.5) and (5.6) results into:

$$
\text { Equilibrium: } \quad p_{\text {ext }}=p_{\text {inf }}=0 \text {. }
$$

The osmotic pressure difference between extra- and intrafibrillar compartments is now given by:

$$
\Delta \pi=\pi_{e x f}-\pi_{\text {inf }}=\pi_{e x f}-\pi_{e x t}=p_{e x f} .
$$

As suggested by Maroudas et al. ${ }^{135}$ the osmotic pressure difference $\Delta \pi=p_{e x}$ is used in the pressure- $\phi_{c i}$ relationship (5.4). Substituting the Donnan osmotic pressure difference ${ }^{223}$ for $\Delta \pi$ we get:

$$
\Delta \pi=\Phi_{t x f} R T\left(2 c_{e x f}^{-}+c_{i x \jmath}^{p g}\right)-2 \Phi_{c x t} R T c_{e x t}
$$

where $\Phi_{e x f}$ is the osmotic coefficient in the extrafibrillar compartment, $R$ is the universal gas constant, $T$ the absolute temperature, $c_{\text {ex }}^{-}$the negative ions concentration in the extrafibrillar compartment, $c_{e x f}^{p g}$ the FCD on extrafibrillar water basis, $\Phi_{e x t}$ the osmotic coefficient of the external solution, and $c_{\text {ext }}$ the external salt concentration. 
$\phi_{c i}$ being dependent on $\Delta \pi(5.4), \Delta \pi$ being dependent on the $c_{e x f}^{p g}(5.11)$, and $c_{e x f}^{p g}$ being dependent on $\phi_{c i}$ because the extrafibrillar water content depends directly on $\phi_{c i}$, an iterative procedure is needed to calculate $\phi_{c i}$, and thus $c_{e x f}^{p g}$. Iteration with eqs. (5.4) and (5.11) converges to a $\phi_{c i}$ with matching $c_{e x f}^{p g}$. Convergence was reached when $\left\|\left(\left(\phi_{c i}\right)^{i}-\left(\phi_{c i}\right)^{i-1}\right) /\left(\phi_{c i}\right)^{i}\right\|$ was smaller than 0.00001 , where $\left(\phi_{c i}\right)^{i}$ is the $\mathrm{i}$-th estimation of $\phi_{c i}$. The extrafibrillar water volume is the difference between total water and intrafibrillar water: $V_{\text {exf }}^{f}=V^{f}-V_{\text {inf }}^{f}$.

The FCD on extrafibrillar water basis. The FCD on extrafibrillar water basis, $c_{e x f}^{p g}$, is given by the number of moles of fixed charges FC, divided by $V_{c x f}^{f}$.

From now on, unless otherwise mentioned, whenever reference is made to $\mathrm{c}^{-}$or $\mathrm{c}^{+}$, the concentrations in the extrafibrillar compartment are meant, and all references to inside the tissue pertain to the extrafibrillar compartment.

The effective second Piola-Kirchhoff stress; stress free elongation factors. The effective second Piola-Kirchhoff stress $S$ is defined as the current effective stress (at time t) in the reference state coordinates (at time $t_{0}$ ). We define the reference situation as the situation at the end of the conditioning phase. Quantities pertaining to the reference situation are marked with a " $0^{n}$ suffix. In a one dimensional situation $S$ is related to the current effective Cauchy stress $\sigma_{e}$ by:

$$
S=\frac{1}{J} \sigma_{e}
$$

To determine $\sigma_{e}$ in the equilibrium states, one needs to calculate the osmotic pressure difference $\Delta \pi$ between the extrafibrillar and external solutions. In equilibrium the externally applied mechanical load $F / A$ is balanced by the extrafibrillar effective Cauchy stress, $\sigma_{e, e x f}$, and the fluid pressure $p_{e x f}$ (see eq. (5.8) with $n$ parallel to the loading direction and the discussion below it):

$$
-F / A=\sigma_{e, e x f}-p_{e x f}=\sigma_{e, e x f}-\Delta \pi .
$$

The last equation comes from the equality of $p_{\varepsilon s f}$ and $\Delta \pi(5.10) . \Delta \pi$ is given in (5.11). To determine the $\Delta \pi$ in equilibrium one needs to know the equilibrium $c^{-}$, and the osmotic coefficients. For the equilibrium $c^{-}$the mean activity coefficients have to be known. The equation for the equilibrium $c^{-}$concentration is derived using electroneutrality, and chemical potential equations.

In the extrafibrillar compartment we have $\mathrm{PG}$, and positive and negative ions. From electroneutrality we have:

$$
c^{+}=c^{-}+c_{e x f}^{p g}
$$

Equilibrium requires that the chemical potentials of the ions are equal for the extrafibrillar and external solutions. This translates to the condition of Donnan equilibrium: ${ }^{126}$

$$
\left(\gamma_{\text {exi }}^{ \pm}\right)^{2} c_{\text {ext }}^{2}=\left(\gamma_{\text {exf }}^{ \pm}\right)^{2} c^{+} c^{-}
$$


with $\gamma_{\text {ext }}^{ \pm}$the mean activity coefficient of the ions in the external solution, $c_{\text {ext }}$ the external molal salt concentration, and $\gamma_{e x f}^{ \pm}$the mean activity coefficient of the ions in the extrafibrillar compartment.

From the electroneutrality condition (5.14), and the Donnan equilibrium condition (5.15), the equilibrium $c^{-}$concentration can be derived:

$$
2 c^{-}=-c_{e x f}^{p g}+\sqrt{\left(c_{e x f}^{p g}\right)^{2}+4\left(\frac{\gamma_{e x t}^{ \pm}}{\gamma_{e x f}^{ \pm}}\right)^{2} c_{e x t}^{2}},
$$

The mean activity coefficients depend on the ions concentrations. For free solutions (salt and water), the mean activity coefficient is tabulated. ${ }^{128,184}$ We used a least squares fit of an exponential/polynomial function of graph data from Maroudas ${ }^{128}$ to determine the mean activity coefficients as a function of ion concentration. For $0.15 \mathrm{M} \mathrm{NaCl} \gamma_{\text {ext }}^{ \pm}$equals 0.755 , for $0.46 \mathrm{M} \mathrm{NaCl}$ it is 0.685 . When the ions concentrations inside can be determined, e.g. via partition studies, the mean activity coefficient inside can be determined from (5.15). Maroudas ${ }^{128}$ found that mean activity coefficients in cartilage lie mostly between 0.65 and 0.72 (external solution $0.15 \mathrm{M}$ ), decreasing when FCD (on total fluid volume) increases.

When the mean internal activity coefficient is not determined experimentally, it can be estimated from semi-empirical equations. The most complete treatment is given by Manning. ${ }^{121}$ Manning's equations apply only to low external concentrations. For higher concentrations, including the physiological, Kwak, ${ }^{106}$ and Wells ${ }^{233}$ suggested modifications. According to these modifications the mean activity coefficient is the product of a poly-ion/mobile ion interaction in the absence of salt, $\gamma^{P M}$, and mobile ion-mobile ion interaction, $\gamma^{M M}$ :

$$
\gamma_{e x f}^{ \pm}=\gamma^{P M} * \gamma^{M M}
$$

Manning ${ }^{121}$ related the $\gamma^{P M}$ to the ratio $X$ of the fixed charge density to the concentration of free electrolyte (i.e. at the concentration of the co-ion) in the tissue:

$$
\ln \gamma^{P M}=-0.5 \xi \frac{X}{X+2},
$$

where the factor $\xi$ has the value 0.99 in accordance with the composition of disc proteoglycans. ${ }^{223} \gamma^{M M}$ is defined as the mean activity coefficient of the salt corresponding to the concentration of the co-ion in the polyelectrolyte solution (Wells ${ }^{233}$ ), or at the mean ionic strength of the polyelectrolyte solution (Kwak, ${ }^{106}$ and Freeman and Maroudas ${ }^{59}$ ). Note that the relationships (5.17) and (5.18) were derived for cartilaginous tissue on the basis of total water volumes.

We estimated the mean activity coefficient and $c^{-}$for the extrafibrillar compartment in absence of determined values of $c^{-}$and $c^{+}$as follows. We used eqs. (5.16)-(5.18) for an iterative procedure to estimate $\gamma_{\text {exf }}^{ \pm}$and $c^{-}$. $\gamma^{M M}$ was calculated using the least squares fitted graph data from Maroudas ${ }^{128}$ at the total mean ionic strength (i.e. $0.5^{*}\left(c_{\text {tot }}^{-}+c_{t o t}^{+}\right)$) of all the ions (intra- plus extrafibrillar) in the total fluid volume, where the subscript "tot" refers to all the ions in the total fluid volume.

$\gamma^{P M}$ was calculated substituting for $X$ the ratio of $c_{e x f}^{p s}$ to the concentration of the co-ion $\left(c^{-}\right)$. Because the proteoglycans are restricted to the extrafibrillar space we used a ratio based on extrafibrillar values here. 
The initial value of $\gamma_{\text {exf }}^{ \pm}$for the iterations was 0.70 . Convergence was reached when $\left\|\left(\left(\gamma_{\text {ex } f}^{ \pm}\right)^{i}-\left(\gamma_{\text {ex }}^{ \pm}\right)^{i-1}\right) /\left(\gamma_{\text {exf }}^{ \pm}\right)^{i}\right\|$ was smaller than 0.00001 , where $\left(\gamma_{\text {ex }}^{ \pm}\right)^{i}$ is the $\mathrm{i}$-th estimation of $\gamma_{e x f}^{ \pm}$. This iteration loop was placed inside the iteration loop for the determination of $\phi_{c i}$ and $c_{e x f}^{p g}$.

In order to calculate values of average activity coefficients that are based on total ions concentrations, one can use the equation:

$$
\left(\gamma_{\text {ext }}^{ \pm}\right)^{2} c_{\text {ext }}^{2}=\left(\gamma_{t o t}^{ \pm}\right)^{2} c_{\text {tot }}^{+} c_{\text {tot }}^{-} .
$$

(5.19) relates the external $\left(\gamma_{\text {ext }}^{ \pm}\right)$and total internal $\left(\gamma_{\text {tot }}^{ \pm}\right)$average activity coefficients using the concentrations $\overrightarrow{c_{t o t}}$ and $c_{t o t}^{+}$based on the total internal number of ions and total fluid volume. Compare (5.19) and (5.15). Note that $\gamma_{t o t}^{ \pm}$does not equal the $\gamma^{ \pm}$that would result if there were no distinction made between extra- and intrafibrillar compartments, because the ions concentrations resulting from one-compartment calculations would not equal those in (5.19). Therefore, $\gamma_{\text {tot }}^{ \pm}$is not more than an indication for the average activity coefficient based on one fluid compartment.

The osmotic coefficient in free $\mathrm{NaCl}$ solution is a function of the $\mathrm{NaCl}$ concentration, ${ }^{128}$ decreasing with increasing concentration. For concentrations of $0.15 \mathrm{M}$ to $0.50 \mathrm{M}$ it is almost constant with a value of 0.924 . The internal osmotic coefficient for cartilaginous tissues is split up in two independent components, viz.:

$$
\Phi_{e x I}=\Phi^{P M} * \Phi^{M M} .
$$

$\Phi^{M M}$ is the osmotic coefficient in aqueous solutions, which is taken either at the co-ion concentration, ${ }^{233}$ or at the mean ionic strength. ${ }^{59,106} \Phi^{P M}$ represents the interactions between the poly-ions and mobile ions. Its dependence on $\gamma^{P M}$ is defined by Manning ${ }^{121}$ as:

$$
\Phi^{P M}=1+\ln \gamma^{P M} .
$$

The $\Phi^{M M}$ was calculated from a least squares fit of an exponential/polynomial function of the graph data from Maroudas, ${ }^{128}$ substituting the total mean ionic strength on total fluid volume basis, analogous to the calculation of $\gamma^{M M}$.

Using the formula:

$$
\pi_{t a x f}=\Phi_{i o t} R T\left(c_{t o t}^{-r}+c_{t o t}^{+}\right) \text {, }
$$

one gets an estimate of the osmotic coefficient $\Phi_{t o t}$ for the total internal fluid volume.

Once the values of $\Phi_{e x t}$ and $\Phi_{e x f}$ have been determined, the $\sigma_{e}$ can be determined from (5.10), (5.11), and (5.13), and $S$ from $\sigma_{e}$ with (5.12).

To derive the $S$ as a function of the Green-Lagrange solid strain $E^{s}$, the 3 data points per experiment were fitted with a linear relationship between $S$ and $E^{s}$ :

$$
\begin{array}{r}
E^{s}=\frac{1}{2}\left(\frac{h^{2}}{h_{s f}^{2}}-1\right), \\
S=H_{e} * E^{s},
\end{array}
$$

where $h$ is the sample's height, $h_{s f}$ is the height of the sample in the stress free state, and $H_{e}$ is the sample's effective linear stiffness. The stress free state is defined as the state where the solid experiences no stress in the loading direction. Note that none of 
the observed experimental states is stress free, because osmotic effects always stress the solid. Therefore, $h_{s}$ could not be determined directly from the experiment, and had to be varied along with the $H_{e}$ to get the best least squares fit. After determination of $h_{s}$ by least squares fitting, stress free elongation factors $\left(J_{s f, i}\right)$ defined as the ratio of the stress free height, divided by a particular height (indicated with subscript "i"), e.g., an equilibrium height, could be calculated.

\section{The biphasic permeability coefficient, $K_{b i}$, and aggregate modulus $H_{A}$}

The biphasic permeability coefficient, $K_{b i}$, and aggregate modulus $H_{A}$ from the compression phase were determined by fitting the analytical solution of linear biphasic compression $^{211}$ to the compression phase, using the least squares criterion. A detailed description of this procedure is given in chapter 4 (Houben et al. ${ }^{83}$ ).

\section{In vivo values of parameters}

To determine approximate in vivo values of several paranneters, we used the sample height measurement at the beginning of the experiment. Measurement was started within 2 minutes after thawing of the sample. The sample height at the beginning of the experiment is thus a measure for the in vivo height, $h_{v}$, of the sample. The parameter values, based on $h_{v}$, are indicated with a subscript " $v$ ". We determined in vivo approximations of the fluid fraction, $\phi_{v}$, the stress free elongation factor, $J_{s f, v}$, the \% of hydroxyproline on wet weight basis, $h y p_{w w, v}$, the FCD on wet weight basis, $c_{w w, v}^{p g}$, and on total fluid volume basis, $c_{v}^{p g}$.

\section{The 1D triphasic computer fit of the CSC experiments}

The equilibrium data of the CSC experiments were used as input data for the $1 \mathrm{D}$ computer implementation of the triphasic model presented in chapter 3 . For an outline of the fit procedure the reader is referred to chapter 3 , section 3.5. The data processing of the equilibrium results of the CSC experiments was based on Donnan osmotic theory, with separate extra- and intrafibrillar fluid compartments. The 1D computer simulation program, however, is based on triphasic theory with only one fluid compartment: no dis-tinction between extra- and intrafibrillar water is made here. Referral to the two (fluid) compartments analysis results of the equilibrium situations of the CSC experiments is made with the terms "equilibrium results". The results of one (fluid) compartment computer simulation are referred to as "(computer) fit results" or "(computer) simulation results". The values of quantities based on total water of fixed charge density, $c_{0}^{p g}$, and fluid fraction, $\phi_{0}^{f}$, at the end of the conditioning stage were calculated from the conditioning equilibrium, and used as fixed input in the simulation. The $J_{s f, 0} H_{c}$ (eq. (5.23)), and $\gamma_{\text {tot, }}^{ \pm}$(eq. (5.19)) values derived from the equilibrium results of the CSC experiments were used as starting values for the computer fit. The biphasic permeabilities, $K_{b i}$, were used as starting values for the triphasic permeabilities, $K_{\text {tri }}$. To calculate the approximate permeability coefficient in the in vivo situation, in vivo hydrations were calculated, and from these hydrations the (approximate) in vivo triphasic permeabilities were calculated, using eq. (3.40), and adding the $\Delta K_{\text {tri }}$ that was determined in the computer fit. We thus 
get:

$$
K_{t r i, v}=0.710 *\left(h y d_{v}\right)^{2}-0.482 * h y d_{v}+\Delta K_{\text {tri }},
$$

where $K_{t r i, v}$ is the estimate of the in vivo triphasic permeability coefficient, and $h y d_{v}$ the approximate in vivo hydration. There were no starting values for the parameter for PG loss, $\frac{d^{p g}}{d t}$, nor for the diffusion coefficient, $D_{t r i}$.

Deformation dependencies of $c^{p g}, \phi^{f}, \gamma_{t o t}^{ \pm}, K_{t r i}$, and $D_{t r i}$ are defined in chapter 3 , section 3.3.1. 


\subsection{Results}

Figure 5.1 shows the curves of the sample height versus time of two experiments. The biphasic fits of the compression stages are given by the dashed lines. Figure 5.la shows the result of an outer sample (distance from mid-sample to annulus edge $0.27 \mathrm{~mm}$ ), Figure $5.1 \mathrm{~b}$ of an inner sample (distance $2.42 \mathrm{~mm}$ ). Comparing Figures $5.1 \mathrm{a}$ and b, we see that the outer sample has a descending conditioning phase, and the inner sample an ascending conditioning phase. The compression phase in Figure 5.1a shows relative to the conditioning equilibrium height more height loss for the outer sample. If we define outer samples as those with distance from mid-sample to annulus edge $\leq 0.6 \mathrm{~mm}$, and inner samples with distances greater than that, we have 4 outer samples, and 19 inner samples. 3 of those 4 outer samples have a descending conditioning phase, 1 of the 19 inner samples has a descending conditioning phase.
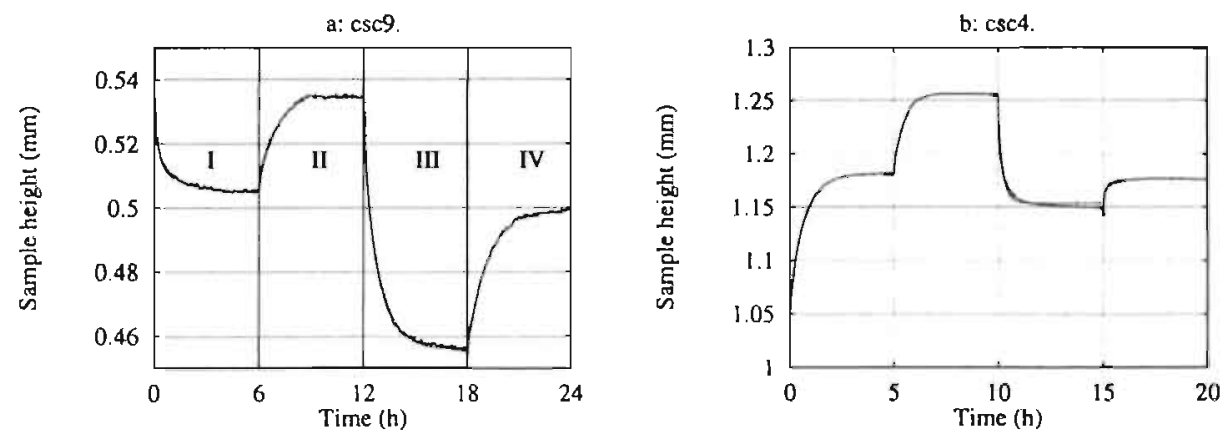

Figure 5.1: Sample height versus time for two experiments: a. csc9, outer sample, b. csc4, inner sample. The dashed line in the compression phase is the biphasic fit. In (a) the 4 stages of the CSC experiment are indicated: $I=$ conditioning, II = swelling, III = compression, IV = control.

\subsubsection{Parameters as a function of distance to the annulus edge}

As a function of the distance of the middle of the sample to the annulus edge we determined the fixed charge density on dry weight basis, $c_{d w}^{p g}$ (Figure $5.2 \mathrm{a}$ ), the biphasic permeability $K_{b i}$ (Figure $5.2 \mathrm{~b}$ ), the biphasic aggregate modulus $H_{A}$ (Figure $5.2 c$ ), the stress free elongation factor relative to the conditioning equilibrium, $J_{s f, 0}$ (Figure $5.2 \mathrm{~d}$ ), and the swelling equilibrium, $J_{s f_{s} s w}$, the $\%$ of hydroxyproline on dry weight basis, $h y p_{d w}$ (Figure 5.2e), and the solid density, $\rho^{s}$. We also calculated as an estimation for the in vivo values the following parameters: the fluid fraction, $\phi_{v}^{J}$, the stress free elongation factor, $J_{s f . w}$, the $\%$ of hydroxyproline on wet weight basis, $h y p_{w w, v}$, the FCD on wet weight basis, $c_{t \prime m, v}^{p g}$, and on fluid volume basis, $c_{v}^{p g}$. These in vivo approximate values are given in table 5.3.

For the biphasic permeability the results of only 15 out of the 23 samples were used, 
a.

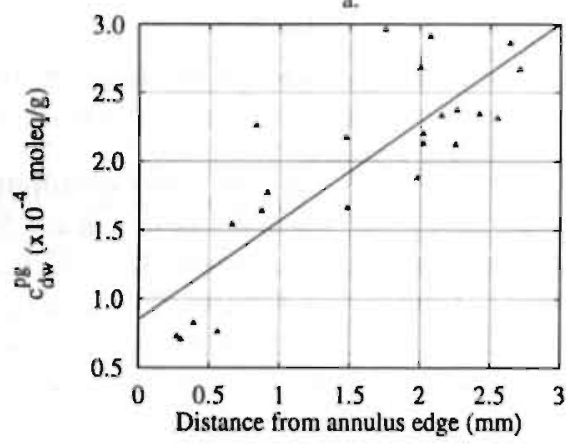

c.

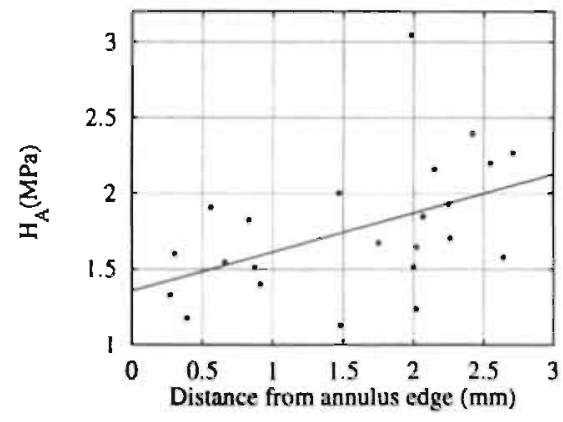

e.

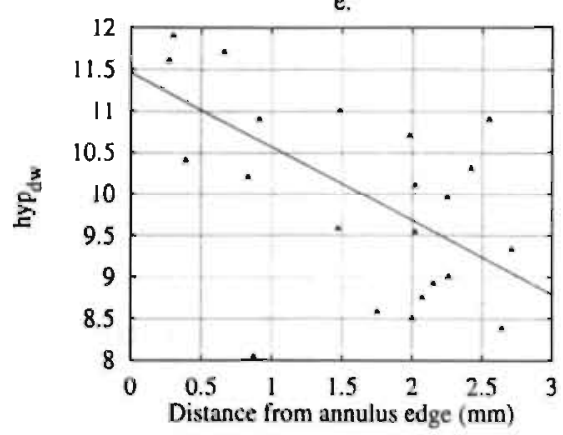

b.

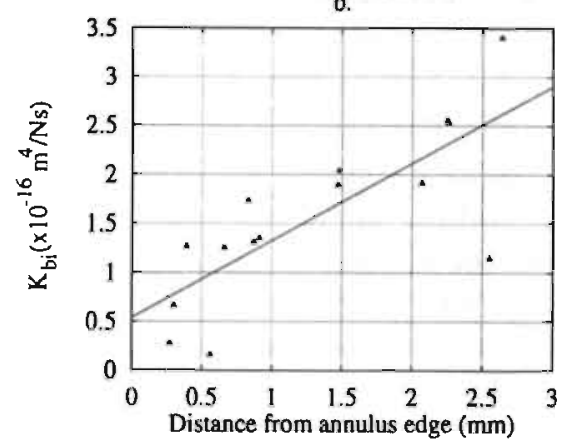

d.

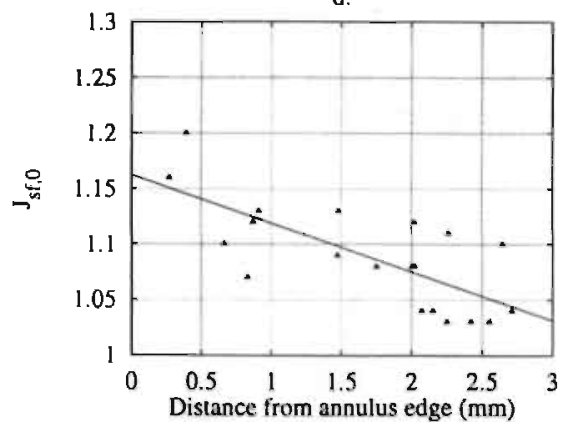

Figure 5.2: FCD an dry weight basis, $c_{d w}^{p g}(a)$, biphasic permeability, $K_{b i}(b)$, biphasic aggregate modulus: $H_{A}(c)$, stress free elongation factor, $J_{, j, 0}$ (d), and \% hydroxyproline on dry weight basis, $h y_{d w}$ (e) as a function of the distance of the center of the sample to the annulus edge. For $e_{d w}^{3 s}, H_{A}$, and $h y p_{d w} \mathrm{n}=23$, for $J_{s f, 0} \mathrm{n}=20$, and for $K_{b i} \mathrm{n}=15$. A reduced number for $J_{s,, 0}$ has been used, because 3 experiments yielded an unphysical stress strain relationship (decreasing strain with increasing stress), and thus no value of $J_{s, 0}$ could be calculated. Only 15 experiments for $K_{b i}$ have been used because of the demand of a relative error in the biphasic time constant of less than $5 \%$. The plots have been fitted with linear least squares fits.

because we required the relative least squares estimation fault of the curve fit to be under $5 \%$. For the stress free elongation factors $J_{s f, 0} J_{s f, s w}$, and $J_{s f_{s},}$ only 20 experiments were used, because 3 experiments yielded effective stress-elongation factor results with a decreasing strain for increasing stress. Therefore, no physically plausible linear stress: 
strain relationship could be fitted, and thus no value of $J_{s /}$ could be calculated.

We see the $c_{d w}^{p g}, K_{b i}$, and $H_{A}$ rise with distance from the annulus edge, and the $J_{s f, 0}$ and $h y p_{d w}$ decrease with distance. The $c_{w w, v}^{p g}$, and $c_{v}^{p g}$ (not plotted) also increased with distance from the annulus edge. We fitted (least squares) the plotted quantities of Figure 5.2 with linear functions, and plotted these functions in the graph. In table 5.2 the coefficients and statistics of the fits are given.

\begin{tabular}{|c|c|c|c|c|}
\hline parameter & $\mathrm{a}$ & $\overline{\mathrm{b}}$ & $\overline{\mathrm{R}}^{2}$ & $p_{a}$ \\
\hline$c_{d w}^{p g}$ & $(7.2 \pm 1.0)^{*} 10^{-5}(\mathrm{moleq} / \mathrm{g}) / \mathrm{mm}$ & $(8.5 \pm 1.8)^{*} 10^{-5} \mathrm{moleq} / \mathrm{g}$ & 0.70 & 0.0000 \\
\hline$K_{b i}$ & $(0.79 \pm 0.18)^{*} 10^{-16}\left(\mathrm{~m}^{4} / \mathrm{Ns}\right) / \mathrm{mm}$ & $(0.54 \pm 0.28)^{*} 10^{-16} \mathrm{~m}^{4} / \mathrm{Ns}$ & 0.59 & 0.0008 \\
\hline$H_{A}$ & $0.26 \pm 0.11 \mathrm{MPa} / \mathrm{mm}$ & $1.36 \pm 0.19 \mathrm{MPa}$ & 0.22 & 0.0243 \\
\hline$J_{s f, 0}$ & $-0.044 \pm 0.010 \mathrm{~mm}^{-1}$ & $1.163 \pm 0.018$ & 0.52 & 0.0003 \\
\hline$h y p_{d w}$ & $-0.89 \pm 0.28 \% / \mathrm{mm}$ & $11.46 \pm 0.50 \%$ & 0.32 & 0.0047 \\
\hline
\end{tabular}

Table 5.2: Linear least squares regression for parameters $\left(\mathrm{n}=23,15\right.$ for $K_{b i}, 20$ for $\left.J_{4,0}\right)$ as a function of distance $d$ in $\mathrm{mm}$ of the mid-sample to the annulus edge: parameter $=\mathrm{a}^{*} d+\mathrm{b}$. In columns 2 and 3 the estimates of the coefficients $\mathrm{a}$ and $\mathrm{b} \pm \mathrm{SD}$ are given. Column 4 lists $\mathrm{R}^{2}$, the coefficient of determination, and finally column 5 gives the two-tailed Student's $t$ probability $p_{a}$, that the slope of the regression line is 0, i.e. that there is no linear relationship between parameter and distance.

For $\phi_{v}^{f}, \rho^{s}, J_{s f, s w}, J_{s f, v}$, and $h y p_{w w, v}$, a linear least squares fit gave no correlation with distance $\left(\mathrm{p}_{a}>0.05\right)$. The average values $\pm S D$, and the extrema of the parameters above, plus those of the distance to the annulus edge and the percentage height loss $\left(\Delta h_{c c}\right)$ of the end of the control phase in \% of the height of the end of the conditioning phase (Figure 5.1) are listed in table 5.3.

\begin{tabular}{|c|c|c|c|}
\hline parameter & average \pm SD & minimum & maximum \\
\hline distance $(\mathrm{mm})$ & $1.59 \pm 0.79$ & 0.27 & 2.71 \\
\hline$c_{d w}^{p g}\left(* 10^{-4} \mathrm{moleq} / \mathrm{g}\right)$ & $1.99 \pm 0.68$ & 0.706 & 2.960 \\
\hline$c_{w u, v}^{\bar{p} g}\left({ }^{*} 10^{-4}\right.$ moleq/g) & $0.80 \pm 0.28$ & 0.283 & 1.170 \\
\hline$c_{v}^{p s}(\mathrm{~mol} / \mathrm{l})$ & $0.135 \pm 0.047$ & 0.0045 & 0.202 \\
\hline$h y p_{d w}(\%$ of dry weight) & $10.1 \pm 1.2$ & $8 . \overline{04}$ & 12.80 \\
\hline$h y p_{w w, v}(\%$ of wet weight) & $4.07 \pm 0.80$ & 2.64 & 6.94 \\
\hline $\bar{K}_{b i}\left({ }^{*} 10^{-16} \mathrm{~m}^{4} / \mathrm{N} s\right)$ & $1.56 \pm \overline{0.84}$ & 0.160 & 3.400 \\
\hline$H_{A}(\mathrm{MPa})$ & $1.77 \pm 0 . \overline{44}$ & 1.127 & 3.044 \\
\hline$H_{\varepsilon}(\mathrm{MPa})$ & $1.09 \pm 0.66$ & 0.208 & 2.550 \\
\hline$J_{s f, 0}$ & $1 . \overline{089} \pm 0.046$ & 1.030 & 1.200 \\
\hline$J_{s\}, s w}$ & $1.013 \pm 0.039$ & 0.961 & $1 . \overline{124}$ \\
\hline$J_{s \delta, v}$ & $1.194 \pm 0.097$ & 0.994 & 1.333 \\
\hline$\phi_{v}^{j}$ & $0.728 \pm 0.068$ & 0.531 & 0.847 \\
\hline$\rho^{s}\left(\mathrm{~g} / \mathrm{mm}^{3}\right)$ & $0.00191 \pm 0.00048$ & 0.00122 & 0.00313 \\
\hline$\Delta h_{c c}(\%)$ & $1.7 \pm 1.2$ & 0.16 & 3.90 \\
\hline
\end{tabular}

Table 5.3: Average \pm SD, and minimum and maximum of the parameter values. $\mathrm{n}=$ 23,15 for $K_{b i}, 20$ for $H_{e}, J_{s, j, i} J_{s f, w}$, and $J_{s, v}$. 


\subsubsection{The mean activity and osmotic coefficients}

For the extrafibrillar mean activity and osmotic coefficients the following average values were found:

conditioning $(\mathrm{n}=23): \gamma_{\text {exf } f}^{ \pm}=0.623 \pm 0.022 ; \Phi_{\text {exf }}=0.801 \pm 0.033$, swelling $(\mathrm{n}=23): \gamma_{\operatorname{exf}}^{ \pm}=0.586 \pm 0.025 ; \Phi_{\text {exf }}=0.710 \pm 0.038$, compression $(\mathrm{n}=23): \gamma_{\operatorname{exf}}^{ \pm}=0.555 \pm 0.024 ; \Phi_{\text {exf }}=0.663 \pm 0.040$, total average $(\mathrm{n}=69): \gamma_{\text {ex } J}^{ \pm}=0.588 \pm 0.037 ; \Phi_{\text {exf }}=0.725 \pm 0.068$.

The estimates for the values of these parameters on total fluid volume using equations (5.19) and (5.22) yielded:

conditioning $(\mathrm{n}=23): \gamma_{\text {tot }}^{ \pm}=0.663 \pm 0.010 ; \Phi_{\text {tot }}=0.879 \pm 0.025$, swelling $(\mathrm{n}=23): \gamma_{\text {tot }}^{ \pm}=0.640 \pm 0.025 ; \Phi_{\text {tot }}=0.850 \pm 0.055$, compression $(n=23): \gamma_{\text {tot }}^{ \pm}=0.623 \pm 0.024 ; \Phi_{\text {tot }}=0.875 \pm 0.103$, total average $(\mathrm{n}=69): \gamma_{\text {iot }}^{ \pm}=0.642 \pm 0.026 ; \Phi_{\text {tot }}=0.868 \pm 0.070$.

Whereas the average activity coefficients are reasonably constant over the stages, there is quite a large variation in osmotic coefficient. From conditioning to compression $\Phi_{e x f}$ shows a decrease of 0.801 to $0.663(17 \%)$ It can be seen that the values on total volume basis for both the activity and osmotic coefficients are higher than their extrafibrillar counterparts. The total average of the mean activity coefficient increases from 0.588 to 0.642 , while the osmotic coefficient increases from 0.725 to 0.868 .

\subsubsection{The intrafibrillar water factor: $\phi_{c i}$}

The iteratively calculated $\phi_{c i}$ values per phase are (mean $\pm \mathrm{SD} ; \mathrm{n}=23$ ): 1 . Conditioning: $\phi_{c i}=1.21 \pm 0.05 \mathrm{~g} / \mathrm{g}, 2$. Swelling: $\phi_{c i}=1.16 \pm 0.04 \mathrm{~g} / \mathrm{g}, 3$. Compression: $\phi_{c i}=1.11 \pm$ $0.05 \mathrm{~g} / \mathrm{g}$. The mean value for all phases is $\phi_{c i}=1.16 \pm 0.06(\mathrm{n}=69)$. The above results indicate that the collagen fibres bind most water in the conditioning phase (highest $\phi_{c i}$ ), and least water in the compression phase.

Taking the factor $\phi_{c i}$ as a function of the osmotic pressure difference, and iteratively calculating it, leads to different solutions of $c_{e x f}^{p g}$ and $V_{e x f}^{f}$, than when $\phi_{c i}$ is taken a constant. We have plotted $c_{e x j}^{p g}$ as a function of the elongation factor $J$ relative to the conditioning state for the iteratively calculated $\phi_{c i}$ (Figure 5.3a), and for a fixed (average of the 3 equilibrium states) $\phi_{c i}$ of 1.16 (Figure 5.3b). In the figure there are per experiment 3 points, joined by 2 line segments. The elongation factor was calculated with reference to the conditioning equilibrium. The swelling equilibrium has a $J>1$, the compression equilibrium has $J<1$. 

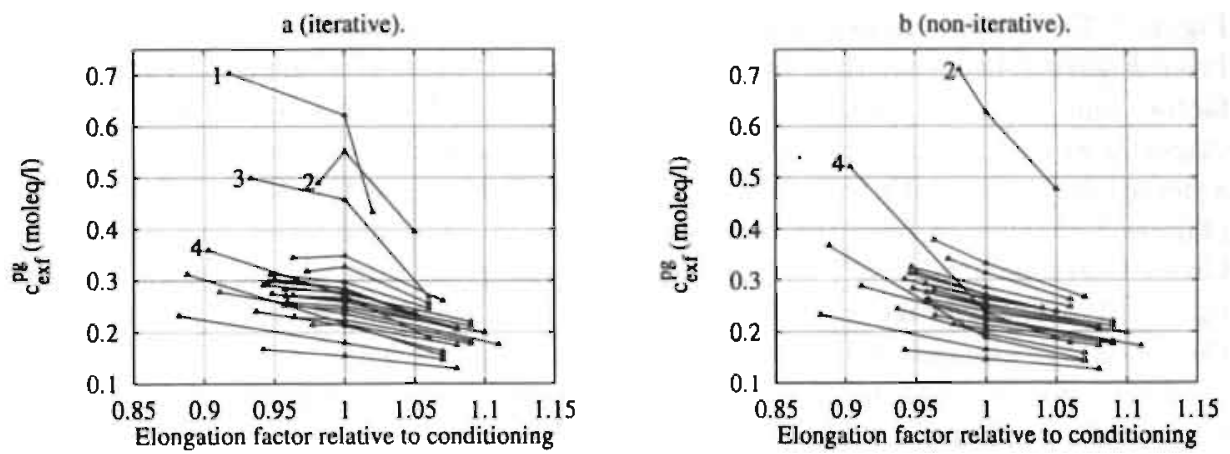

Figure 5.3: The $e_{e x s}^{n g}$ as a function of the elongation factor $J$ relative to the conditioning height. In (a), $\phi_{\mathrm{ci}}$ is iteratively calculated for each phase separately (indicated with the term "iterative" in the header). In (b), $\phi_{c i}$ is taken as the average of the iteratively calculated $\phi_{c i}$ of the 3 equilibrium stages: 1.16 (indicated with the term "non-iterative" in the header). For comparison labels 1-4 are placed in (a), and labels 2 and 4 in (b). Experiments 1 and 3 are missing from (b) because application of the constant $\phi_{c i}$ of 1.16 resulted in unphysical results for these experiments.

In Figure 5.3 we see that the results for iteratively (a) and non-iteratively (b) calculated $\phi_{c i}$ differ. One difference is that the $c_{e x f}^{p g}$ values from the compression equilibrium for non-iteratively calculated $\phi_{c i}$ are higher. For comparison labels $1-4$ have been placed in Figure 5.3a, and in 5.3b labels 2 and 4. Experiments 1 and 3 are missing from the constant $\phi_{c i}$ (non-iterative; b) results because the results were unphysical.

\subsubsection{Effective stress}

The effective 2nd Piola-Kirchhoff stress $S$ was calculated for the 3 equilibrium states. In Figure $5.4 \mathrm{a} S$ is given as a function of the elongation factor. The same 4 experiments

a.

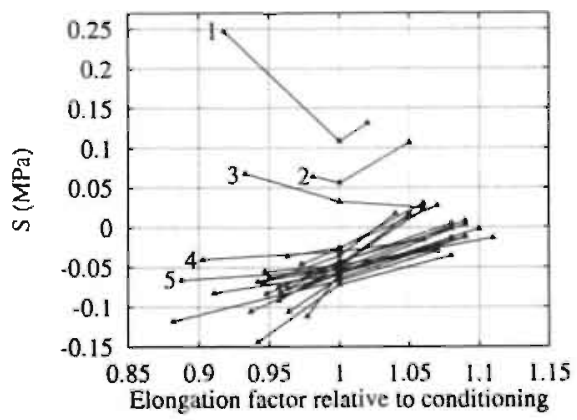

b.

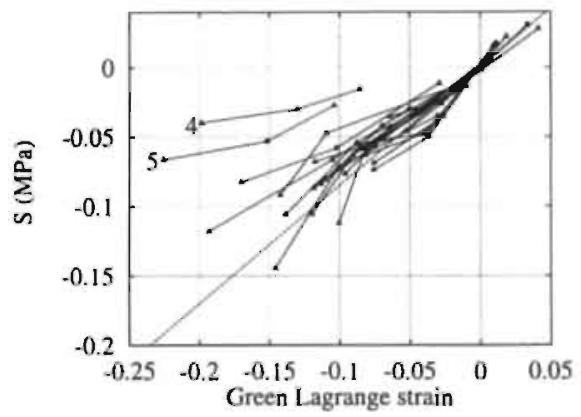

Figure 5.4: (a) The second Piola-Kirchioff stress $S$ as a function of the elongation factor for all experiments $(n=23)$. (b) $S$ versus the Green-Lagrange strain for a linear fit of the experiments that had an increasing stress, with strain $(n=20)$. The average linear fit (doited line) is also plotted in $b$ (slope $=$ $1.09 \mathrm{MPa}$ ). Outliers have been labelled. Experiments $1-3$ are left out in $\mathrm{b}$ because these yielded negative regression coefficients.

as in Figure 5.3 have been labelled, plus experiment 5. Experiments 1-3 are missing from 
Figure $5.4 \mathrm{~b}$ because these yielded negative regression coefficients.

From Figure 5.4a we see that the effective stress increases as a function of the elongation factor, and that it is negative for the compression $(J<1)$ and conditioning $(J=1)$ stages in all but 3 experiments. Experiments 1-3 show from compression to conditioning a decreasing stress with an increasing $J$. They all have positive stress values. We fitted (Figure 5.4b; dashed line) the 20 experiments with increasing stress as a function of the Green-Lagrange strain, $E^{s}$, with a linear function going through the origin according to eq. (5.23). The average $\pm \mathrm{SD}$ values $(\mathrm{n}=20)$ for the effective linear stiffness, $H_{e}$, and the stress free elongation factors, $J_{s f, i}$, are given in table 5.3.

There was a slight increase of $H_{\epsilon}$ from outer to inner annulus. Linear regression yielded: $H_{e}=(0.160 \pm 0.053)^{*} d+0.448 \pm 0.098 \mathrm{MPa}$, where $d$ is the distance from the annulus edge in $\mathrm{mm} ; \mathrm{R}^{2}=0.33, \mathrm{p}_{a}=0.0076$. For the 8 paired samples, the inner sample always had a bigher $H_{\epsilon}$.

\subsubsection{The 1D triphasic computer fits of the CSC experiments}

In chapter 3 the results of two computer fits of CSC experiments are shown. In this section the individual results of 6 more experiments are shown, as well as the triphasic permeability coefficient results of the computer fits (for $n=15$, and $n=23$ ), and the effective stiffness and stress free heights $(n=20)$. In Figure 5.5 the graphs of sample height versus time for the $6 \mathrm{CSC}$ experiments, with their computer simulations are shown. Tables 5.4-5.6 show the parameter values of the 6 CSC experiments. In table 5.4 the mechanical and chemical loads are given. 

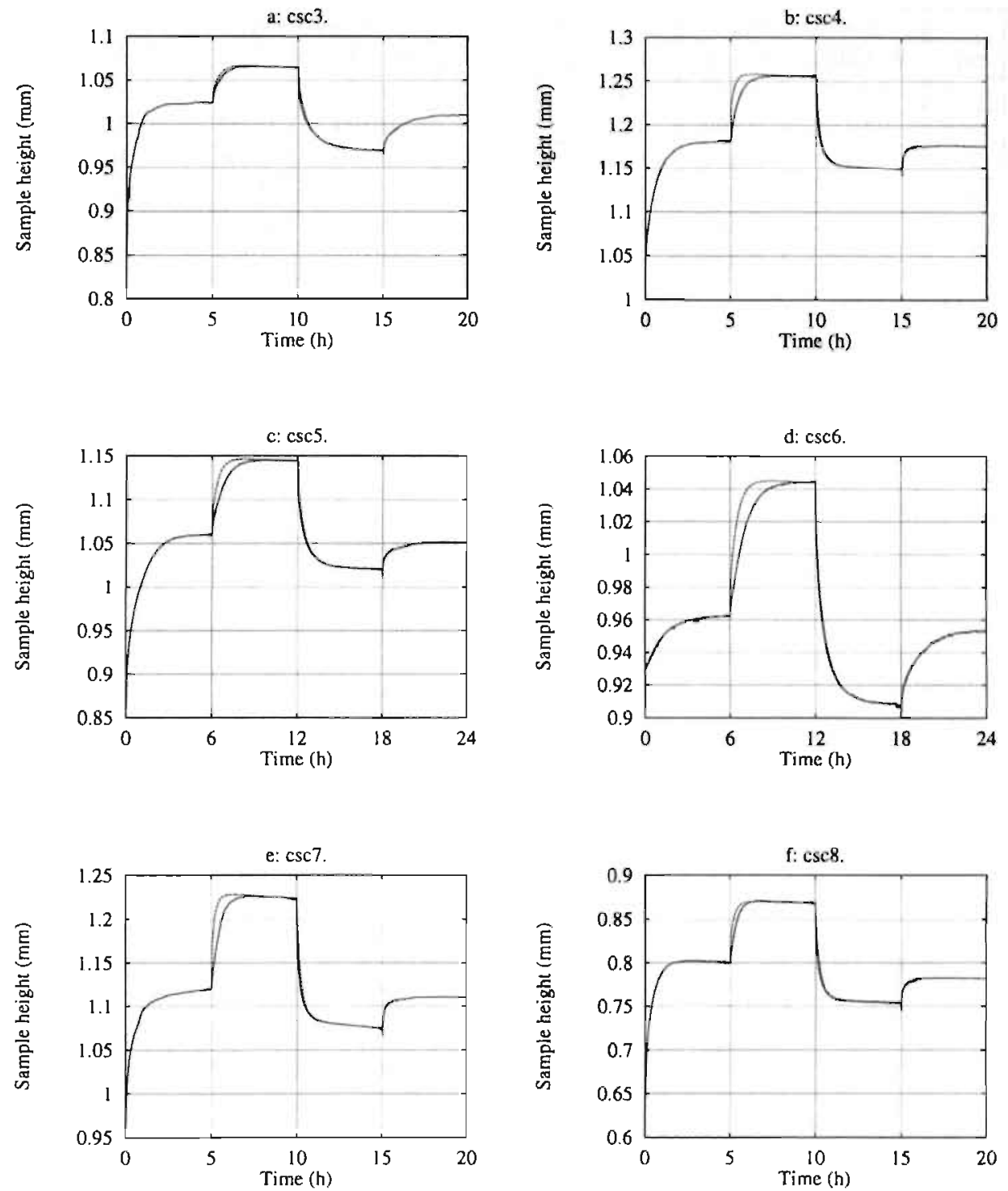

Figure 5.5: Triphasic computer model fits of the swelling and compression phases of six CSC experiments ( $\csc 3-\csc 8)$. The solid line shows the experiment, the dashed line the fit. 


\begin{tabular}{|c||c|c||c|c||c|c||c|c|}
\hline \multicolumn{1}{|c||}{} & \multicolumn{2}{c||}{ conditioning } & \multicolumn{2}{c||}{ swelling } & \multicolumn{2}{c||c}{ compression } & \multicolumn{2}{c}{ control } \\
\hline exp. & W (MPa) & $\mathrm{C}(\mathrm{M})$ & W $(\mathrm{MPa})$ & $\mathrm{C}(\mathrm{M})$ & W $(\mathrm{MPa})$ & $\mathrm{C}(\mathrm{M})$ & W $(\mathrm{MPa})$ & $\mathrm{C}(\mathrm{M})$ \\
\hline csc3 & 0.0778 & 0.447 & 0.0778 & 0.167 & 0.194 & 0.167 & 0.0778 & 0.447 \\
\hline $\csc 4$ & 0.0772 & 0.460 & 0.0772 & 0.148 & 0.193 & 0.148 & 0.0772 & 0.460 \\
\hline $\operatorname{csc5}$ & $\mathbf{0 . 0 7 7 2}$ & 0.458 & 0.0772 & 0.146 & 0.193 & 0.146 & 0.0772 & 0.458 \\
\hline $\operatorname{csc6}$ & 0.0772 & 0.477 & 0.0772 & 0.161 & 0.193 & 0.161 & 0.0772 & 0.477 \\
\hline $\operatorname{csc7}$ & 0.0778 & 0.480 & 0.0778 & 0.164 & 0.194 & 0.164 & 0.0778 & 0.480 \\
\hline $\csc 8$ & 0.0772 & 0.480 & 0.0772 & 0.164 & 0.193 & 0.164 & 0.0772 & 0.480 \\
\hline
\end{tabular}

Table 5.4: The mechanical (W) and chemical loads $(\mathrm{C}: \mathrm{M}=\mathrm{mol} / \mathrm{l})$ of $6 \mathrm{CSC}$ experiments, $\csc 3-\csc 8$.

For $c_{0}^{p g}, \frac{d c^{p g}}{d t}, \phi_{0}^{J}, J_{s f, 0}$ and $H_{e}$, both values determined from the equilibrium states of the experiment ("eql."; used as starting values for the computer fit), and final values determined from the computer fit ("fit"), are shown in table 5.5 .

\begin{tabular}{|c||c||c||c||c|c||c|c|}
\hline & \multicolumn{1}{|c||}{$c_{0}^{p g}(\mathrm{moleq} / \mathrm{l})$} & $\frac{d c^{p g}}{d t}\left(\mathrm{moleq} /\left(\mathrm{l}^{*} \mathrm{~h}\right)\right)$ & \multicolumn{1}{c||}{$\phi_{0}^{f}$} & \multicolumn{2}{|c||}{$J_{s f, 0}$} & \multicolumn{2}{c|}{$H_{e}(\mathrm{MPa})$} \\
\hline exp. & eql. \& fit & fit & eql. \& fit & eql. & fit & eql. & fit \\
\hline csc3 & 0.127 & $-0.89^{*} 10^{-3}$ & 0.82 & 1.027 & 1.004 & 0.97 & 1.21 \\
\hline $\csc 4$ & 0.142 & $-0.56^{*} 10^{-3}$ & 0.72 & 1.029 & 1.013 & 0.90 & 1.12 \\
\hline $\operatorname{csc5}$ & 0.126 & $-0.42^{*} 10^{-3}$ & 0.76 & 1.071 & 1.035 & 0.76 & 0.88 \\
\hline $\csc 6$ & 0.090 & $-0.28^{*} 10^{-3}$ & 0.85 & 1.119 & 1.077 & 0.91 & 0.87 \\
\hline $\csc 7$ & 0.153 & $-0.56^{*} 10^{-3}$ & 0.78 & 1.085 & 1.006 & 0.74 & 0.67 \\
\hline $\csc 8$ & 0.152 & 0 & 0.77 & 1.077 & 0.979 & 0.63 & 0.54 \\
\hline
\end{tabular}

Table 5.5: Parameter values for the CSC fit: "eql." indicates the values determined from the equilibrium data of the experiments, "fit" indicates the values determined from the computer simulation. The subscript " 0 " refers to the conditioning equilibrium.

\begin{tabular}{|l||c|c||c|c||c|c|}
\hline \multicolumn{1}{|c||}{} & \multicolumn{3}{c||}{$K_{\text {tri }}\left({ }^{*} 10^{-16} \mathrm{~m}^{4} / \mathrm{Ns}\right)$} & \multicolumn{2}{c||}{$D_{\text {tri }}\left({ }^{*} 10^{-7} \mathrm{~m}^{2} / \mathrm{s}\right)$} & \multicolumn{2}{|c|}{$\gamma_{\text {tol }}^{ \pm}$} \\
\hline exp. & swel. & compr. & swel. & compr. & swel. & compr. \\
\hline $\csc 3$ & 1.79 & 1.35 & 4.93 & 4.59 & 0.681 & 0.678 \\
\hline $\csc 4$ & 4.53 & 4.12 & 3.41 & 3.06 & 0.680 & 0.676 \\
\hline $\operatorname{csc5}$ & 2.63 & 2.03 & 4.09 & 3.64 & 0.685 & 0.680 \\
\hline $\csc 6$ & 2.09 & 1.44 & 5.75 & 5.29 & 0.696 & 0.690 \\
\hline $\csc 7$ & 6.31 & 5.51 & 4.45 & 3.94 & 0.676 & 0.671 \\
\hline $\csc 8$ & 3.71 & 3.01 & 4.20 & 3.65 & 0.676 & 0.670 \\
\hline
\end{tabular}

Table 5.6: The equilibrium values of $K_{t r i}, D_{t r i}$ and $\gamma_{i o t}^{ \pm}$of the swelling and compression phases determined from the computer simulation.

The equilibrium values for the swelling and compression stage: of the fit for $K_{t r i}$, $D_{t r i}$, and $\gamma_{t o t}^{ \pm}$are given in table 5.6. The values for $D_{t r i}$ in table 5.6 were calculated using the formula of Mackie and Meares, ${ }^{119}$ equation (3.42). For all the simulations a $D_{f r e e}^{\mathrm{NaCl}}$ of $1^{*} 10^{-7} \mathrm{~m}^{2} / \mathrm{s}$ was chosen, which is 62 times the value of the diffusion of $\mathrm{NaCl}$ in an aqueous solution $\left(1.61^{*} 10^{-9} \mathrm{~m}^{2} / \mathrm{s}\right)$.

From the computer fits we determined the triphasic permeahility coefficients during the swelling and compression stages for the same 15 experiments as were used for the deter- 

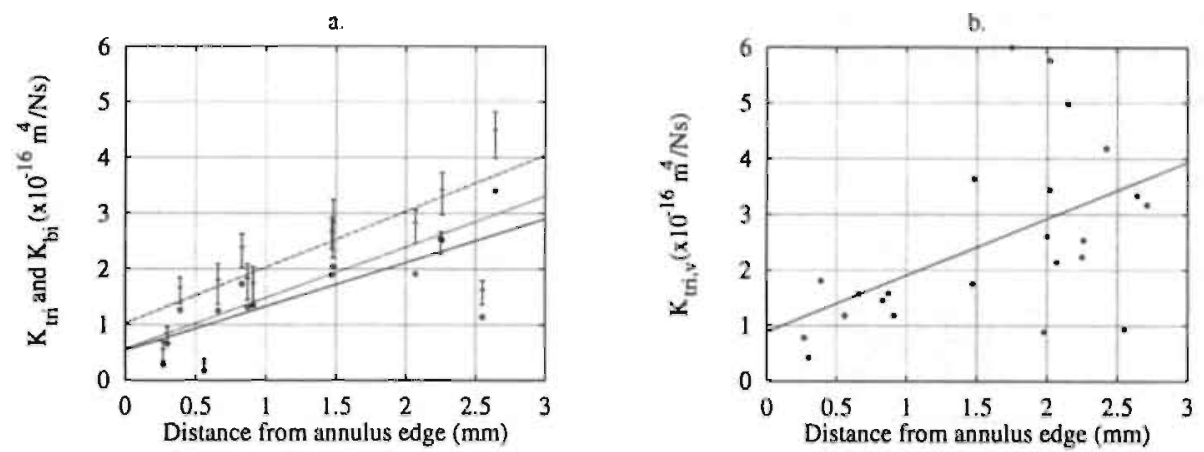

Figure 5.6: (a) the bi- and triphasic permeability coefficients, $K_{b i}(0)$, and $K_{t r i}$ (error-bars) $(\mathrm{n}=15)$ as a function of distance of the mid-sample to the annulus edge. The extrema of the triphasic permeability coefficient correspond with the swelling (maximum) and compression (minimum) equilibrium values of the fit. The bottom solid line corresponds with a linear regression through the biphasic results, the middle dotted line with the compression $K_{t r i, c}$, and the top dot-dashed line with the swelling $K_{\text {tri,s. }}$ (b) the in vivo triphasic permeability coefficients, $K_{t r i, v}$, as a function of the distance of the mid-sample to the annulus edge, along with the linear regression fit $(n=23)$.

mination of the biphasic permeability coefficients. The maximum values for the triphasic permeability coefficients in the computer simulation are found during the swelling equilibrium, the minima during the compression equilibrium. The averages over the swelling and compression stages, and the extrenna are shown in Figure 5.6a. For comparison, the biphasic results are reproduced in Figure 5.6a. The in vivo triphasic permeability coefficients, $K_{\text {tri, }}$, were determined for all 23 experiments. All compression stages of the experiments could be fitted satisfyingly with the computer simulation (in contrast to the determination of $K_{b i}$, for which only 15 experiments passed). The result of $K_{t r i, v}$ as a function of distance of the mid-sample to the annulus edge is given in Figure 5.6b.

The average values of the permeability coefficients $(n=15)$ are: triphasic swelling: $K_{\text {tri,s }}=(2.3 \pm 1.1)^{*} 10^{-16} \mathrm{~m}^{4} / \mathrm{Ns}$, triphasic compression: $K_{\text {tri, }}=(1.8 \pm 1.0)^{*} 10^{-16} \mathrm{~m}^{4} / \mathrm{Ns}$, biphasic: $K_{b i}=(1.6 \pm 0.8)^{*} 10^{-16} \mathrm{~m}^{4} / \mathrm{Ns}$.

The averages for the in vivo triphasic permeabilities, for $n=15$, and $n 1=23$ are: triphasic in vivo $(\mathrm{n}=15): K_{t r i, v}=(1.8 \pm 0.9)^{*} 10^{-16} \mathrm{~m}^{4} / \mathrm{Ns}$, triphasic in vivo $(\mathrm{n}=23): K_{t r i, t}=(2.5 \pm 1.5)^{*} 10^{-16} \mathrm{~m}^{4} / \mathrm{Ns}$.

From Figure $5.6 \mathrm{a}$ it can be seen that for most experiments the triphasic permeabilities of both the swelling and compression equilibria are greater than the biphasic permeability. Of the averages, only the $K_{t r i, s}$ is significantly greater than $K_{b i}$ (2-tailed, exact $\mathrm{P}=$ 0.0408 ; Mann-Whitney). In Figure 5.6a and $\mathrm{b}$ we also see that the permeability coefficients rise with distance from the annulus edge:

swelling: $K_{\text {tri,s }}=(1.01 \pm 0.26)^{*} d+(1.02 \pm 0.40) ; \mathrm{R}^{2}=0.54 ; p_{a}=0.0017$ compression: $K_{\text {tri,c }}=(0.91 \pm 0.21)^{*} d+(0.57 \pm 0.32) ; \mathrm{R}^{2}=0.60 ; \mathrm{p}_{a}=0.0007$, biphasic: $K_{b i}=(0.79 \pm 0.18)^{*} d+(0.54 \pm 0.28) ; \mathrm{R}^{2}=0.59 ; \mathrm{p}_{a}=0.0008$, triphasic in vivo $(\mathrm{n}=23): K_{\text {tri,v }}=(1.02 \pm 0.36)^{*} d+(0.89 \pm 0.65) ; \mathrm{R}^{2}=0.27 ; \mathrm{P}_{a}=$ 0.0109 , where $K_{\text {tri }}, K_{6 i}$ are in units of $10^{-16} \mathrm{~m}^{4} / \mathrm{Ns}$, and $d$ is the distance of the mid- 
sample to the annulus edge in $\mathrm{mm} . \mathrm{p}_{a}$ is the probability of rejection that the slope of the regression line does not equal zero. We see that all permeability coefficients rise signifcantly with distance to the annulus edge on a $5 \%$ level.

\begin{tabular}{|c|c|c|c|}
\hline & eql. & fit & P \\
\hline$J_{\text {sf }, 0}$ & $1.089 \pm 0.046$ & $1.033 \pm 0.039$ & 0.0003 \\
\hline$H_{e}(\mathrm{MPa})$ & $1.09 \pm 0.66$ & $0.83 \pm 0.18$ & 0.1344 \\
\hline$\gamma_{\text {tot, }}^{ \pm}$ & $0.640 \pm 0.025$ & $0.687 \pm 0.012$ & 0.0000 \\
\hline$\gamma_{\text {tot, }, \text { _ }}^{ \pm}$ & $0.623 \pm 0.024$ & $0.681 \pm 0.013$ & 0.0000 \\
\hline
\end{tabular}

Table 5.7: Comparison of values from equilibrium analysis of CSC experiments ("eql."), with values, determined from the computer fit ("fit"). The 2-tailed, exact P-values are given in the right column. $\gamma_{t o t}^{ \pm}$, is the average value of $\gamma_{t o t}^{ \pm}$for the swelling equilibrium state, $\gamma_{t o t, c}^{ \pm}$ditto for the compression equilibrium. For $J_{s,, 0}$ and $H_{e} \mathrm{n}=20$, for $\gamma_{\text {tot, }}^{ \pm}$and $\gamma_{\text {iot,c }}^{ \pm} \mathrm{n}=23$.

the equilibrium values from the experiments.

Linear regression of $J_{s f, 0}$ as a function of the distance of the mid-sample to the annulus edge, $d$, through the computer fit results $(\mathrm{n}=20)$ resulted in:

$J_{s f, 0}=(-0.040 \pm 0.007)^{*} d+(1.000 \pm 0.010) ; \mathrm{R}^{2}=0.62 ; \mathrm{p}_{a}=0.0000$.

For the equilibrium results the linear fit for $J_{s f, 0}$ was:

$J_{s f, 0}=(-0.044 \pm 0.010)^{*} d+(1.163 \pm 0.018) ; R^{2}=0.52 ; \mathrm{p}_{a}=0.0003$.

From table 5.7 we can see that the values of $J_{s f, 0}$ for the fit are significantly lower than their equilibrium counterparts. The same holds for the average activity coefficients. The average $H_{e}$ for the fit is smaller than that, for the equilibrium results, but because of the large standard deviation, there is no significant difference. 


\subsection{Discussion}

This study introduces a protocol to determine mechanical properties of IVD annulus using porous media concepts and Donnan osmosis (triphasic theory; Lai et al., ${ }^{109}$ Snijders et al. ${ }^{203}$ ), dividing the fluid compartment into intra- and extrafibrillar compartments based on the findings of Maroudas and colleagues, ${ }^{93,130,135,232}$ and Urban and McMullin. ${ }^{224,225}$ This extension increases the number of constituents in the mixture from 3 to 4 (an extra ions phase in the intrafibrillar compartment). The possibility to derive a linear relationship for the effective stiffness has been demonstrated. The average linear effective stiffness between the effective 2nd Piola-Kirchboff stress and Green-Lagrange strain was found to be $1.087 \pm 0.657 \mathrm{MPa}$.

The introduction of extra- and intrafibrillar compartments, requires a new interpretation of ionic activity and osmotic coefficients. Determinations in the past of these quantities ${ }^{126,128,131}$ have been based on Donnan osmotic theory for the total fluid compartment. This means that new experiments have to be designed to determine quantities such as $\gamma^{M M}, \gamma^{P M}, \Phi^{M M}$, and $\Phi^{P M}$ for the extrafibrillar compartment. The laws that relate the above quantities to fixed charge density and ions concentrations (see equations (5.16)-(5.18), (5.20), and (5.21)) are semi-empirical and the interpretation of these laws in terms of what concentrations should be substituted has been subject of discussion. ${ }^{28}$ We used concentrations $\left(c^{+}+c^{-}\right) / 2$ based on the total volume for the determination of $\gamma^{M M}$ and $\Phi^{M M}$, whilst for the poly-mobile ions interactions we chose concentrations on the basis of the extrafibrillar compartment. With this choice, the 2nd PK effective stress increased as a function of Green-Lagrange strain.

From the results of the coefficients per equilibrium stage we infer that especially the osmotic coefficient is quite sensitive to changes in the chemical and mechanical loads. These results indicate that for the determination of the osmotic coefficient it is important to accurately define the experimental conditions.

It appears that the activity coefficients determined for the extrafibrillar compartment are smaller than the literature values for cartilage. For the mean activity coefficient for the ions in cartilage Maroudas found for an external solution of $0.15 \mathrm{M} \mathrm{NaCl}$ the values to be in the range of $0.65-0.72 .{ }^{128}$ For the extrafibrillar compartment we found an average over the 3 equilibrium stages of $0.588 \pm 0.037$. After correction for the total volume the value of $0.642 \pm 0.026$ was found which lies at the bottom of the range found by Maroudas for cartilage. Note, however, that the correction for total volume does not correspond with directly determining quantities for one fluid compartment, as clarified in the methods section. Further differences are that the mean activity coefficient for the ions from our experiments is inferred from canine data in an experiment where mechanical loads were applied. For the determination of activity coefficients in cartilage, no mechanical load was applied in Maroudas' experiments. ${ }^{131}$

For the osmotic coefficient we found for the extrafibrillar and total volume values respectively: $0.725 \pm 0.068$ and $0.868 \pm 0.070$. Maroudas ${ }^{126}$ inferred from cartilage data the relationship

$\Phi_{\text {cartilage }} / \Phi_{\text {ext. sointion }} \approx 0.8$. For an external $\Phi_{e x t}$ of 0.942 (which is the value for molalities over $0.15 \mathrm{~mol} / \mathrm{kg}$ ) this would mean an internal osmotic coefficient of $\approx 0.74$, which lies closest to our extrafibrillar value. We have no explanation why in this case the osmotic coefficient for cartilage lies closer to the extrafibrillar value. 
The results of the stress-strain relationship proved to be quite sensitive to the ionic activity and osmotic coefficients, and the pressure- $\phi_{c i}$ relationship. It is therefore important that these quantities in future studies be determined accurately. E.g., activity coefficients (for a single fluid compartment) can be determined from partition studies.

\subsubsection{Position dependent parameters}

Fixed charge density, hydroxyproline, and water content. To our knowledge, no data on $F C D$ of canine IVD were available prior to this study. All fixed charge density quantities $\left(c_{d w}^{p g}, c_{w w, v}^{p g}, c_{v}^{p g}\right)$ increased with distance to the annulus edge. For the fixed charge density on wet weight basis we found an average of $(0.80 \pm 0.28)^{*} 10^{-4} \mathrm{moleq} /(\mathrm{g}$ wet weight). Urban and Maroudas ${ }^{219}$ found for human intervertebral discs in the outer regions of the annulus values between $0.7^{*} 10^{-4}$ and $1.3^{*} 10^{-4} \mathrm{moleq} /(\mathrm{g}$ wet weight). They also found an increase of $c_{w w, v}^{p g}$ going from the outer annulus inwards.

For hydroxyproline and collagen we found relatively high values: hydroxyproline on dry weight basis $10.1 \pm 1.2 \%$. On wet weight basis the estimate of the hydroxyproline content in vivo was $4.07 \pm 0.86 \%$. For a conversion factor of 7.55 the collagen average on dry weight basis was: $75.9 \pm 9.4 \%$. Ghosh et al. ${ }^{64}$ examined collagen content in canine IVD as a function of age, spinal level and breed. They found no variation of collagen content with age, or spinal level for the AF. For chondroclystrophoid and nonchondrodystrophoid breeds they found the AF collagen content to be around $55 \%$ (using a conversion factor of 7.4). Skaggs et al. ${ }^{199}$ found for human lumbar annulus fibrosis for the outer anterior annulus $62.6 \pm 7.7 \%(\mathrm{n}=9)$, for the inner anterior annulus $59.3 \pm$ $6.0(\mathrm{n}=8)$, for the outer posterior annulus $63.0 \pm 12.9 \%(\mathrm{n}=9)$, and for the inner posterior annulus $66.6 \pm 5.9 \%(\mathrm{n}=9)$. They found no correlation with radial position for collagen. Best et al. ${ }^{17}$ calculated for human discs the hydroxyproline per wet weight. They reported a decrease of hydroxyproline per wet weight from outer to inner annulus, and lower values of hydroxyproline in the dorsal-lateral regions compared to the ventral region. The overall mean value on wet weight basis was $2.5 \pm 1.0 \%$.

The increase of $c_{w w, v}^{p g}$ with distance from the annulus edge is in accordance with the findings of Urban and Maroudas. ${ }^{219}$ For the $\%$ hydroxyproline ( $\%$ collagen) there is no uniformity in experimental studies. We found a decrease with distance to the annulus edge of $h y p_{d w}$, but no correlation for $h y p_{i w w, v}$, whilst, albeit for human discs, Best et al. found a decrease of $h y p_{w w, y}$. Skaggs et al. ${ }^{199}$ found no correlation of $h y p_{d w}$ with radial position.

We did not find a radial dependence of water content $\left(\phi_{v}^{f}\right)$ for the pooled results of all the samples. It is however generally accepted that the water content of AF decreases from outer to inner annulus. ${ }^{17,199,219}$ Of the 5 pairs of neighbouring samples (10 out of 23 samples) we found 4 with higher $\phi_{v}^{f}$ of the inner sample, corroborating the trend of increasing water content from outer to inner annulus. The absence of radial dependence of the pool of samples might therefore be due to a large scatter in the values of hydration per disc and disc region.

The results above indicate that it is best to compare hydroxyproline values on a dry weight basis, because water content has been shown to have large fluctuations between discs and regions, so as to obscure variation as a function of radial position. 
Biphasic permeability coefficient and aggregate modulus Our average biphasic permeability coefficient $K_{b i}$ was $(1.56 \pm 0.84)^{*} 10^{-16} \mathrm{~m}^{4} / \mathrm{Ns}$. The radial permeability coefficient of human disc nucleus and annulus was determined by Urban and Maroudas. ${ }^{222}$ They also used creep data to determine the permeability coefficient, and equilibrated the samples in a salt solution (molarity is not indicated) prior to testing. As a function of hydration they found values for annulus from (0.5-11.5)*10 $10^{-16} \mathrm{~m}^{4} / \mathrm{Ns}$ (results from one disc), with permeability rising with hydration. It is not clear, however, what measure they took for the hydration. They used the "original hydration", but this could be the hydration of the sample prior to the equilibration in the salt solution corresponding to the in vivo value, or after the equilibration, just before the application of the load.

We did not find a correlation between the permeability coefficient and in vivo hydration, but did find an increase of the permeability coefficient with swelling equi-

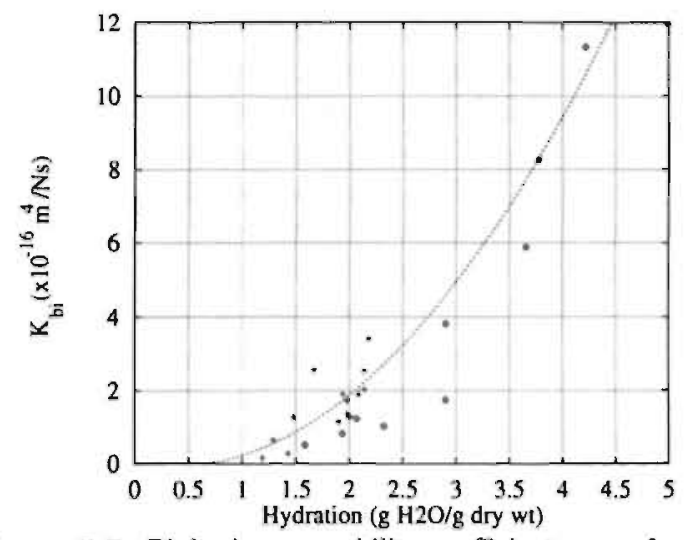

Figure 5.7: Biphasic permeability coefficients as a function of hydration: 0 : data from Urban and Maroudas, ${ }^{222}$ + : data from the present experiments using swelling equilibrium hydrations. The dashed line represents a least squares fit for the data from the present experiments: $y=$ $0.734 * x^{2}-0.948 * x$

annulus specimens (from outer to inner annulus) and found $K_{b i}=(2.5 \pm 1.1)^{*} 10^{-16}$ $\mathrm{m}^{4} / \mathrm{Ns}$. We can conclude that the order of magnitude of our canine $K_{b i}$ corresponds with those of human annulus measured by Urban and Maroudas, ${ }^{222}$ and the human biphasic permeability coefficients determined by Best et al..

We again found a decrease of $K_{b}$ with distance from the outer annulus edge, as in earlier experiments, ${ }^{\overline{8} 3}$ as can be seen in Figure $5.2 \mathrm{~b}$. This finding is not corroborated by the findings of Best et al. ${ }^{17}$ In another study by Houben et al. ${ }^{83}$ possible explanations for this discrepancy is given. The nature of the samples (intact edge versus non-intact edge, canine versus human), and the experimental protocols used (constant strain by Best et al. versus constant stress by Houben et al.) are the most plausible.

The biphasic aggregate modulus $H_{A}$ was found to increase from outer to inner samples, i.e. inner samples were (biphasically) stiffer than outer ones under the combination of $0.078 \mathrm{MPa}$ mechanical load and $0.159 \mathrm{M}$ chemical load (the loads in the swelling phase). This increased stiffness of inner samples can well primarily be caused by a higher osmotic 
stiffness of inner samples, as their $c^{p g}$ is higher than that of outer samples. In a previous study, Houben et al. ${ }^{83}$ found that the biphasic aggregate modulus (compressive modulus) was greater for outer samples. Best et al. ${ }^{17}$ found a trend for the outer samples to be stiffer. In Houben et al. these aggregate moduli were determined from a compression from an equilibrium state of $0.08 \mathrm{MPa}$ and $0.16 \mathrm{M}$. Best et al. used varying mechanical loads $(0.02-0.11 \mathrm{MPa})$ and $0.15 \mathrm{M} \mathrm{NaCl}$. We compared the $H_{A}$ of the outer samples of the previous biphasic study, ${ }^{83}$ with that of the outer samples of this study (distance of mid-sample to annulus edge $<1 \mathrm{~mm} ; \mathrm{n}=8$ ), and found that these values did not differ significantly: $H_{A}=1.56 \pm 0.34 \mathrm{MPa}\left(\mathrm{n}=24\right.$; biphasic previous study) versus $H_{A}=1.54$ $\pm 0.23 \mathrm{MPa}\left(\mathrm{n}=8\right.$; this study). The inner samples had significantly higher values $\left(\mathrm{p}_{a}\right.$ $=0.004): 1.31 \pm 0.47(\mathrm{n}=13$; previous study) versus $1.89 \pm 0.47 \mathrm{MPa}(\mathrm{n}=15$; this study).

Using eq. (5.13) to determine the relative contribution of the extrafibrillar effective (auchy stress $\sigma_{e, t r f}$, and the extrafibrillar fluid pressure, $p_{e x f}$, to the bearing

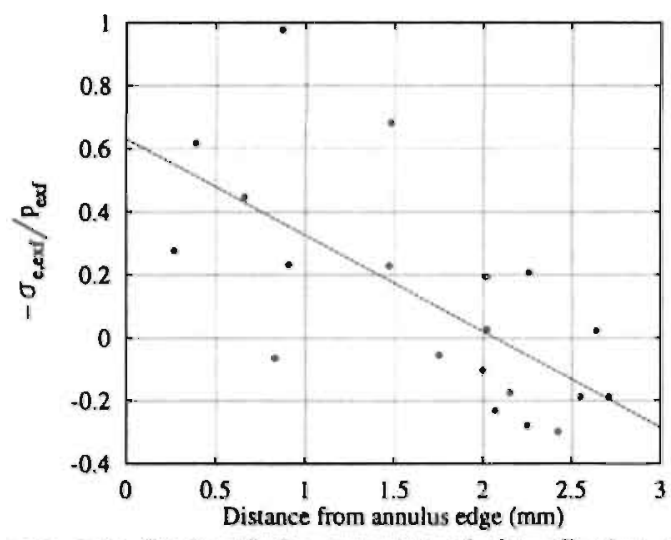

Figure 5.8: Ratio of the negative of the effective extrafibrillar Cauchy stress $\left(-\sigma_{e, e x j}\right)$ and the fluid pressure in the extrafibrillar compartment $\left(p_{e x j}\right)$ for the swelling equilibrium phase as a function of the distance of the mid-sample to the annulus edge. A linear fit yielded: $y=$ $-0.305^{*} \mathrm{x}+0.631, \mathrm{R}^{2}=0.457, \mathrm{P}=0.0011$. weight. We also found that the absolute value of the effective Cauchy stress decreased, and that the extrafibrillar fluid pressure increased for the swelling phase equilibrium going from the outer annulus inward.

The increased aggregate modulus (total stiffness) of inner versus outer samples is thus caused by the increase of osmotic pressure. Whether the aggregate modulus increases or decreases from the outer annulus inward depends therefore on the absolute and relative magnitudes of the effective and osmotic stresses, both a function of radial position. It should be borne in mind that the effective and osmotic stiffnesses both depend on the deformation of the tissue, and that these parameters do not per se correlate with distance from the annulus edge in every equilibrium state. In fact, the swelling equilibrium state was the only state where we found a correlation. 


\subsubsection{Effective stress-strain law and stress free state of the sam- ple}

A linear relation between the radial stress and strain has been fitted, resulting in an average effective stiffness coefficient $H_{e}$ of $1.09 \pm 0.66 \mathrm{MPa}(\mathrm{n}=20)$. In order to obtain a relationship between the stress and strain, the stress free height of the sample, i.e. the height where the solid is stress free in the loading direction, also had to be fitted. This height corresponds with zero strain. From Figure $5.4 \mathrm{~b}$ it can be seen that most GreenLagrange strains were negative. This means that in most equilibrium states the solid was under compression. For the sample's state at the beginning of the experiment, which is an approximation of the in vivo state, the stress free elongation factor $J_{s j, v}$ was greater than unity (table 5.3) for 19 out of 20 experiments, meaning that the stress free height of the sample was greater than the approximate in vivo height. Thus it seems that in vivo the solid is under compression in radial direction. Note that this conclusion is based on the supposition that the stress-strain relationship (2nd PK stress and Green-Lagrange strain) is linear. For other relationships this is not necessarily true.

A remarkable finding is that $H_{e}$ increased from the outer annulus inwards. Also for the paired samples the inner sample always had a greater $H_{\epsilon}$ than the outer one. In tensile tests of AF it was found by Galante ${ }^{61}$ for annulus specimens in circumferential direction, and by Skaggs et al. ${ }^{199}$ for single lamelar AF specimens that the stiffness decreased going from the outer annulus inwards. However, these results refer to tensile (mainly solid) stiffness in the circumferential direction of the annulus, and not to compressive stiffness in the radial direction. Furthermore, it is impossible to determine the effective stiffness $H_{e}$ by direct measurement, because in a direct measurement the sample's stiffness is determined by the effective stress and fluid pressure. The effective stress $\sigma_{e}$ equals the total stress minus the fluid pressure, and is not equal to the intrinsic solid stress, because the $\sigma_{e}$ is based on the total mixture surface.

We have opted for the linear approach as a first and simple approximation for the determination of the effective stress because we had only 3 equilibrium points to infer a relationship from. Further research into the elastic response of the material is needed to reveal a more exact relationship. Getting more data points per experiment to fit a stress-strain curve is however difficult because of the long equilibration times needed per point, and the deterioration (loss of $\mathrm{PG}$ and autolysis) of the sample in the course of the experiment.

\subsubsection{The influence of $\phi_{c i}$ on calculated results}

We calculated the $\phi_{c i}$ by using a curve fit of the pressure- $\phi_{c i}$ relationship based on articular cartilage data from Maroudas et al. ${ }^{135}$ because we had no pressure- $\phi_{c i}$ relationship for IVD AF. As pressure variable the osmotic pressure difference between the extra- and intrafibrillar compartments was taken, as was suggested by Wachtel and Maroudas. ${ }^{231}$ However, we had different experimental conditions (confined swelling and compression) than those under which Maroudas and colleagues determined the cartilage pressure- $\phi_{a i}$ relationship. They either used unconfined compression, or osmotic compression in dialysis sacs.

We found an average value of $1.16 \pm 0.06(n=69)$ for the 3 equilibrium states for $\phi_{c i}$, 
which compares reasonably with the value of 1.33 determined by Urban and McMullin. ${ }^{224}$ From the results of Figure 5.3 we can conclude that the extrafibrillar $c^{p g}$ is susceptible to changes in $\phi_{c i}$. We also compared results using much lower values of $\phi_{c i}\left(\phi_{c i}=0.86\right)$, and found that there was much less scatter in the stress-strain results, and that all 23 2nd PK effective stresses were negative. In view of the importance of a well determined $\phi_{c i}$ for the understanding of IVD mechanics, further research into $\phi_{c i}$-pressure relationships under various experimental situations for IVD is necessary.

\subsubsection{The 1D triphasic computer fits of the CSC experiments}

Fitting the CSC experiments with the computer simulations has been found to be a. relatively easy task, once an equilibria analysis has been performed. With measured values for the fixed charge density, and the fluid fraction, the equilibrium heights of the swelling and compression stages could be fitted by varying the stress free length ratio, $J_{s f, 0}$ the effective stiffness, $H_{c}$, and the PG loss parameter, $\frac{d c^{p g}}{d t}$. The values of $J_{s f, 0}$ and $H_{e}$, derived from the equilibrium (two-fluid-compartment) results of the CSC experiments were good starting values for the fit of the equilibria in the computer simulation (table 5.7). The stress free elongation factors from the fit were systematically smaller (around $5 \%$ ) than the results from the equilibrium analysis. The $H_{\epsilon}$ of the fit were normally around $20 \%$ smaller than the $H_{\epsilon}$ of the equilibrium analysis of the experiments.

The biphasic permeability coefficients were good estimators for the triphasic permeability coefficients. $K_{b i}$ was systematically around $14 \%$ smaller than $K_{\text {tri,s. }}$. The average activity coefficient of the ions was calculated automatically according to the dependencies of Manning. ${ }^{121}$ The estimate of its starting value (from the conditioning phase) had no influence on its values during the swelling and compression equilibria in the computer simulation, because these values were iteratively calculated, concurrently with the values of the ions concentrations. The activity coefficient of the fluid was kept constant at a value of 1 , which was no impediment for fitting the experiments.

The results of Figure 5.5 show that the equilibria of the swelling and compression stages, as well as the compression transient were fitted very well. The swelling transient, however, could not be fitted concurrently with the compression transient. It was possible, by decreasing the value of $K_{t r i}$, to get a reasonable fit of the swelling transient, but even then the value of the diffusion coefficient was higher than the value of the diffusion coefficient for diffusion of $\mathrm{NaCl}$ in an aqueous solution $\left(1.61^{*} 10^{-9} \mathrm{~m}^{2} / \mathrm{s}\right)$. A high diffusion coefficient slows down the swelling of the material in response to a step change in the external salt solution. The finding that extremely high (unphysical) diffusion coefficients do not result in a decrease in swelling speed indicates that the triphasic model, albeit qualitatively correct, is not capable of quantitatively predicting swelling transient behaviour. The triphasic model ignores the Donnan potential, which is present across the boundary of a medium with fixed charges in contact with a salt solution. This Donnan potential might play a role in slowing down the swelling of the porous medium in response to a step change in the external salt solution.

Biphasic and triphasic permeability coefficients The triphasic permeability coefficients were found to be greater than the biphasic ones. The fact that $K_{b i}$ and $K_{\text {tri }}$ are different is not surprising; $K_{b i}$ is based on the gradient in the fluid pressure, $\nabla p$, while 
$K_{t r i}$ is based on the gradient of the fluid pressure minus the osmotic pressure, $\nabla(p-\pi)$. A greater $K_{t r i}$ (assurning equal fluid flows) would thus mean that $\|\nabla p\|>\|\nabla(p-\pi)\|$. All permeability coefficients $\left(K_{t r i, s}, K_{t r i, c}\right.$, and $\left.K_{t r i, v}\right)$ rose with distance of the mid-sample to the annulus edge. This finding corroborates the conclusion of chapter 4 that in vivo permeabilities do rise with distance to the annulus edge. However, some caution is warranted with this conclusion: it should be borne in mind that in vivo hydrations, and thus the $K_{t r i, v}$, are only approximately known, because (1) measurements did not always start right after the thawing of the samples; they started somewhere between 0 and 2 minutes, and (2) the assumption that the sample state right after the thawing in the CSC setup represents the in vivo state is an approximation.

Activity coefficients As was reported in chapter 3, a value of 1 for the activity coefficient of the fluid, $\gamma^{f}$, was chosen. This value, which corresponds with an infinitely diluted solution, was chosen for lack of data on this coefficient. The fit results (especially the equilibrium heights) were quite sensitive to changes in this parameter. The osmotic coefficient (not defined in the numerical formulation) is linked to the activity coefficient of the fluid, but can not be directly calculated from it. It is thus impossible to use the values of the osmotic coefficients, calculated from the equilibrium results, for the determination of $\gamma^{J}$. Because of the sensitivity of the fit results to $\gamma^{j}$ (different values of $\gamma$ lead to different values of $J_{s J, 0}$ and $H_{\epsilon}$ ), it is important to determine this parameter independently.

The average activity coefficient of the ions, $\gamma_{\text {tot }}^{ \pm}$, was found to be reasonably close to the values found from the equilibrium analysis. The values of the average activity coefficient were calculated using the equations of Manning. ${ }^{121}$ The attentive reader will have noticed that the input for the equations of Manning was not the same for the (twofluid-compartments) equilibrium analysis, and the (one-fluid-compartment) computer fit (compare sections 3.3.1 and 5.3.4). This can be justified by remarking that the average activity coefficients for the two-fluid-compartments theory are based on extrafibrillar values of the ions concentrations, while for the one-fluid-compartment theory, they are based on total fluid volume ions concentrations. Separation of the fluid compartment in an extra- and intrafibrillar compartment requires a new definition of activity coefficients for the ions in dependence of the extrafibrillar concentrations of these ions.

\subsubsection{Conclusions}

Confined swelling and compression experiments can be combined with a number of physico-chemical measurements to separate the elastic, osmotic, and viscous contributions of annulus fibrosus to the overall behaviour of the intervertebral disc. Results have been observed to be highly sensitive to values of activity and osmotic coefficients. To obtain a reliable set of chemo-mechanical parameter values, it is important that those parameters that can be measured independently, are measured independently. E.g., the activity coefficients for the ions can be measured from partition studies, although this method only yields results for a single fluid compartment. Also, the dependence of the fraction of intrafibrillar water on the various loading conditions, mechanical and chemical will have to be revealed. The combination of triphasic mixture theory with intra- and 
extrafibrillar compartments has been proven to yield results that differ from the triphasic view with one fluid compartment.

Results of the in vivo triphasic permeability coefficients indicate that this coefficient increases, as does the biphasic permeability coefficient, from outer to inner annulus.

After equilibrium analysis, the computer simulation based on one-fluid-compartment triphasic theory has been used to fit the swelling and compression equilibria, and compression transient of a CSC experiment. The triphasic model, however shows shortcoming in the fitting of the swelling transient. A thorough investigation into the determination of fluid and ions activity coefficients, based on two-fluid-compartments models will have to be conducted to get more insight in the separation of solid (stress free dimensions, effective stiffness) and osmotic contributions to the mechanical behaviour of porous swelling materials.

In view of the fact that the existence of two different fluid compartments has been proven incontestably by Maroudas and others, ${ }^{135}$ it seems that integration of the two compartments into mixture theory is necessary for the correct describing of chemo-mechanical loading of cartilaginous tissues.

\section{Acknowledgements.}

The authors wish to thank Dr. Jill Urban for her advise concerning FCD measurements, and Paul Willems and Arjan Frijns for their assistance with the experiments. 


\section{Chapter 6}

General discussion, conclusions and recommendations 


\subsection{Introduction}

At present, it is commonly accepted that cartilaginous tissues such as the intervertebral disc do not primarily sustain mechanical loads by means of elastic and viscous forces, ${ }^{145,146,190,192,195,197,204,206}$ but that osmotic, or alternatively, electrochemical forces fulfill an important role in the mechanical behaviour. ${ }^{109,125,128,200,221-223}$ For the understanding of the etiology of disc diseases (e.g. disc herniation), and the process of disc degeneration (aging), it is of vital importance to separate the various components that contribute to the mechanical behaviour of the disc. E.g., in order to know the stresses the solid fibres experience, the magnitude of the osmotic pressure has to be known.

Also, for the construction of artificial intervertebral discs, a detailed knowledge of the different constituents is required. Only then the mechanical properties of the real discs can be mimicked.

In the light of the requirements above this study has focused on (1) the development of experimental methods and a protocol to separate the elastic, osmotic, and viscous contributions to the overall mechanical behaviour of the IVD, and (2) to develop a numerical formulation and computer program to implement these features in a numerically economic fashion.

A protocol to determine the separate chemo-mechanical properties from confined swelling and compression (CSC) experiments, and measurements of fixed charge density (FCD) and hydroxyproline, is presented in chapter 5. With this protocol it is possible to find a relationship between the effective stress (a measure for the solid stress), and the strain. The radial variation of the permeability and other quantities in the disc is investigated in chapters 4 and 5. A model and numerical formulation based on the chemical potentials of fluid and salt molecules, with continuous boundary values are presented in chapters 2 and 3 , respectively.

In this chapter the most important findings of this study are discussed. Topics of discussions of previous chapters are summarized, or broadened. Recommendations for future research in the field of swelling mechanics of porous, charged media, with specific reference to the canine intervertebral disc annulus fibrosus, are given where appropriate.

\subsection{The determination of chemo-mechanical quanti- ties of the IVD AF}

\subsubsection{Fitting the equilibrium situations of the CSC experiments using Donnan osmosis and introducing extra- and intrafib- rillar fluid compartments}

This thesis presents a protocol to determine chemo-mechanical parameters of radial samples of annulus fibrosus (chapter 5). CSC experiments and measurements of water content, FCD, and hydroxyproline served as a basis for the determinations. The analysis of equilibrium situations of the CSC experiments was based on the separation of the fluid compartment in an intra- and extrafibrillar compartment. Donnan osmotic theory was applied to determine osmotic pressures. Using the semi-empirical relations of Manning, ${ }^{121}$ values for the average ions activity coefficients, and osmotic coefficients were calculated. 
The size of the extrafibrillar compartment was calculated based on the osmotic pressure difference between the compartments, using articular cartilage data of Maroudas. ${ }^{135}$ Concentrations, activity coefficients, and osmotic coefficients in the intrafibrillar compartment were assumed to equal those in the external bath. Among the results were the effective stress and stress free height of the samples. The effective stress equals the solid stress times the solid volume fraction when there is no fluid flow. The stress free height yields information about the dimensions of the unstressed solid fibres, relative to the in vivo situation. Approximate in vivo values were determined from extrapolations of the equilibria data, making use of the sample height at the beginning of the CSC experiment, which is a measure of the in vivo height.

\section{Extra- and intrafibrillar fluid compartments; activity and osmotic coefficients} The separation of cartilaginous tissue water into extra- and intrafibrillar water compartments was postulated by Maroudas and colleagues. They demonstrated that osmotic forces in cartilaginous tissues can be predicted from osmotic data of pure proteoglycan solutions, if it is assumed that part of the water is shielded from the proteoglycans by the collagen. This part is called the intrafibrillar part. ${ }^{93,130,135,232}$ This finding upsets the view of a single fluid compartment, on which current bi- and triphasic models are based. ${ }^{109,195,203,205}$ In order to incorporate the two-fluid-compartments model into mixture models of cartilaginous tissues, it is essential to (1) determine the size of the extrafibrillar compartment accurately, and (2) to define separate activity coefficients for fluid and ions, as well as osmotic coefficients, in the intra- and extrafibrillar fluid compartments. With regards to (1) Maroudas and colleagues determined the size of the intrafibrillar compartment for cartilage by means of a coefficient, $\phi_{c i}$, that gives the weight of intrafibrillar water in g per $g$ of collagen. ${ }^{135}$ It was concluded that $\phi_{c i}$ depends on the pressure that is applied to the cartilage sample. $\phi_{c i}$ decreases, both for increase in mechanical and increase in osmotic pressure. From unconfined mechanical and osmotic compression experiments they deduced a pressure- $\phi_{c i}$ relationship, which could be fitted with an exponential function. However, neither for intervertebral disc, nor under the circumstances under which we performed our experiments (confined swelling and compression), do data on $\phi_{c i}$ exist. Hence, firstly, experiments need to be done to reveal the pressure- $\phi_{c i}$ relationship for IVD under various combinations of mechanical and chemical loads.

Once a pressure- $\phi_{c i}$ relationship for IVD has been established, and two-fluidcompartments theory is used in the context of a mixture model, it is not possible to use the definitions of activity coefficients and the osmotic coefficient that are based on a single fluid compartment. The activity coefficient for ions is defined to indicate the fraction that is "active", i.e., chemically not inert. Alternatively, it is defined to account for non-ideality of the Donnan osmotic theory. ${ }^{33}$ For a uni-univalent salt solution (e.g. NaCl) the average activity coefficient decreases with increasing concentration. For cartilaginous tissues, the average activity coefficient of $\mathrm{NaCl}$, defining a single fluid compartment, can be obtained from partition studies assuming an ion distribution according to the Donnan theory. ${ }^{13 !}$ With the introduction of extra- and intrafibrillar compartments, the average activity coefficient of the salt in the extrafibrillar compartment differs from that of the intrafibrillar compartment. In the intrafibrillar compartment there are no PG, with which the ions can interact. Therefore, a simple partition study does not suffice to determine the average ions activity coefficient in the extrafibrillar compartment: assumptions concerning the division 
of ions activity between the two compartments have to be made. A first approximation would be to treat the intrafibrillar compartment equivalent to the external solution. In the interpretation of intra-tissue osmotic coefficients we are confronted with the same problem.

Lacking data on the pressure- $\phi_{c i}$ relationship, and the extrafibrillar activity and osmotic coefficients, assumptions had to be made concerning their values and dependencies on concentrations. The results, however, demonstrate that it is possible with this limited knowledge, to get an estimate of the various chemo-mechanical parameters.

\subsubsection{The computer fit of the swelling and compression stages of the CSC experiments with a single fluid compartment}

Next to the equilibrium analysis, that was based on two fluid compartments, a computer fit of the swelling and compression stages of the CSC experiments was performed, using the numerical formulation of chapter 3 , and defining only one fluid compartment: concentrations of ions and FCD were calculated based on the total fluid volume. Equilibrium analysis data, and biphasic permeability coefficients were used as starting input for the computer simulation. The two-fluid-compartments equilibrium analysis results were found to be good starting values for the fit (section 5.4.5). However, both the equilibrium analysis, and the computer fit were performed with incomplete knowledge of the parameter values (osmotic and ions average activity coefficients for the equilibrium analysis, the fluid activity coefficient for the computer fit). This means that at this point the exact quantitative difference between a one-fluid-compartment and a two-fluid-compartments approach is not known.

It was easy to fit the swelling and compression equilibria with the computer simulation, as well as the compression transient. However, the computer simulations of CSC experiments in chapters 3 and 5 (sections 3.5 and 5.4.5, Figures 3.4 and 5.5, show a faster swelling transient than was found in reality. The diffusion coefficient, $D_{\text {tri }}$, had to be chosen so (unrealistically) high (in order to slow down the swelling response) that further increase of $D_{t r i}$ did not result in slowing down the swelling speed.

The problem, encountered in fitting the swelling transient may have its origin in the fact that the streaming and diffusion potentials in the triphasic model are neglected, as is discussed below. The definition of only one fluid compartment in the computer model, in stead of two, is also a possible cause for the fast swelling response of the model.

The approach used in the numerical model in this thesis for tissue swelling is based on the assumption that the driving forces for salt molecules movement and fluid flow are proportional to the respective gradients of their chemical potentials. Assuming a dilute solution, the gradient of the fluid chemical potential equals the gradient of the hydrostatic fuid pressure $p$ minus the Donnan osmotic pressure $\pi$. Hence the dependence of fluid flow and thus swelling behaviour on Donnan osmotic pressure.

The generation of an electrical potential gradient in the mixture due to polarization of the ionic species during fluid flow, or ion diffusion, is not accounted for in the present work. It is arguable, that the introduction of an electrical potential field, or equivalently, the separate electrochemical potential for cations and anions, slows down the swelling response in the simulation, because of the viscous drag the counterions (cations) exert on the fluid. In order to account for the electrical potential, the chemical potential of 
the salt molecules thus should be replaced by electrochemical potentials of the separate ions. ${ }^{179}$ The introduction of the electrochemical potential for the separate ions changes the triphasic theory into a quadriphasic theory.

The use of chemical expansion next to Donnan osmosis to predict swelling pressures as introduced by Lai et al. ${ }^{109}$ seems superfluous, as Donnan osmotic theory alone, albeit with the use of osmotic and activity coefficients, is capable of quantitatively predicting swelling pressures (chapter 5). The problern, however, remains that the osmotic and activity coefficients need to be determined. For one-fluid-compartment theory, the Poisson-Boltzmann model may be used to estimate activity and osmotic coefficients in the Donnan model. ${ }^{33}$ For two-fluid-compartments theory, it remains to be seen whether there is a simple solution to the problem.

\subsubsection{The CSC experiment as an instrument to determine chemo- mechanical quantities of the intervertebral disc}

The confined swelling and compression experiment is a useful tool to determine chemomechanical properties of cartilaginous tissues, especially IVD tissue. Firstly, the exact geometry of the sample is known at all times, so that FCD and fluid fraction can be calculated at any given moment, as a function of the deformation. Secondly, the mechanical and chemical loads are applied in a controlled fashion, so that the loads are also known at all times. Despite the evident advantages of the CSC experiment to test IVD tissue, one should be aware of the limitations of the method.

In vivo parameter values from in vitro CSC experiments In the procedure for the preparation of samples that were tested in the CSC setup, it was the aim to safeguard the in vivo characteristics. The spinal columns were removed within a few hours after death. After removal, the columns were frozen at $-65^{\circ} \mathrm{C}$, and the material was kept frozen during the various stages of treatment. In such a deep-frozen state, the mechanical properties of articular cartilage have been proven to stay the same for several months. ${ }^{107}$ Deep frozen storage time of the material was kept within a maximum of one month.

As a result, the sample is expected to be in its in vivo state at the beginning of the CSC experiment, after thawing. Its height is the in vivo height. Fluid content, fixed charge density, and permeability coefficients have in vivo values. The CSC experiment can thus be used to determine in vivo values of chemo-mechanical parameters, and assess models that include tissue swelling.

The starting procedure of a CSC experiment, however, takes some time: valves have to be opened, air has to be removed from the tubing. To avoid measurement of noise from the starting procedure, measurement of the sample height was sometimes delayed 1 or 2 minutes, as a result of which the initially measured sample height did not equal the in vivo height, but was an approximation of this height.

Loss of PG Because of the long times needed for equilibration of the material (5-10 h for samples of thickness 0.7-1.5 mm), the experiment duration is so long that loss of PG (due to autolysis) is unavoidable..$^{17}$ Because the minimum number of equilibrium situations is 3 , the minimum time needed for an experiment is $15 \mathrm{~h}$. Within $15-20 \mathrm{~b}$ the loss in height 
of the samples (as a result of material loss) is usually within reasonable bounds (chapters 4 and 5), but after $30 \mathrm{~h}$, severe loss of material has occurred. The number of equilibrium situations that can be realized is thus limited to 4 (this includes the control phase). This leaves for the determination of the effective stress-strain relationship according to the protocol described in chapter 5 a maximum number of 3 points per experiment.

The height loss in the control phase of the experiment is expected to be mainly due to the reduced capacity of the tissue to attract water by means of osmosis, because of loss of fixed charges, and not so much because of the direct loss of solid material (PG molecules). The reason for this is the fact that PG molecules bind amounts of water that exceed the volume of the PG molecule itself many times: e.g., the average fluid fraction of the annulus samples of chapter 5 is 0.73 .

The loss of fixed charges was modeled in chapter 4 (section 4.5.5) as a linear loss in sample height at $5 \mathrm{~h}(4.5 \mathrm{~h})$ after the beginning of the experiment. Results for $K_{b i}$ and $H_{A}$ were compared with and without linear correction of sample height decrease. Values of permeability coefficients and aggregate moduli when including the linear loss differed only a few per cent from values when neglecting PG loss. For the computer fit of CSC experiments in chapter 5 (section 5.4.5) a linear decrease of $c_{\text {tot }}^{p g}$, beginning at the end of the conditioning phase $\left(5\right.$ or $6 \mathrm{~h}$ ) was assumed. ${ }^{\text {a }}$ This improved the quality of the fits.

The one-dimensional nature of the CSC experiment A limitation of the CSC experiment is the fact that it is a one dimensional method. The three-dimensional constitutive behaviour of the material can not be evaluated by means of one-dimensional tests. The samples are assumed homogeneous in properties, while IVD tissue is in fact very inhomogeneous. One thus gets an average of the mechanical properties over the volume of the sample. The results of the CSC experiments are dependent on the orientation of the sample in the $\operatorname{disc}^{51}$ (e.g., in the direction of the fibres, perpendicular to the fibre direction).

\subsubsection{Conclusions}

The procedure used in chapter 5 for the determination of chemo-mechanical parameters from equilibrium situations of CSC experiments demonstrates that it is possible to distill solid and osmotic properties separately from CSC experiments, dividing the fluid between extra- and intrafibrillar compartments. The computer model (chapter 3) of the triphasic mixture theory with Donnan osmosis was found to fit equilibrium situations and compression transients of CSC experiments easily. However, swelling transients could not be fitted properly. The diffusion coefficient was unrealistically high. An important problem in using the Donnan osmotic model for tissue swelling is the determination of activity and osmotic coefficients. The separation of the fluid compartment into intra- and extrafibrillar compartments poses extra problems, both theoretically and experimentally, with regards to the determination of separate activity and osmotic coefficients for the two compartments. How should the intrafibrillar compartment be defined? Do the values of activity and osmotic coefficients of the intrafibrillar compartment correspond with their

aNote that linear correction for sample height loss as performed in chapter 4 does not necessarily correspond with a linear decrease in $c_{\text {tot }}^{p g}$ as assumed in chapter 5. 
counterparts in the external bath? We did not find indications for the introduction of a chemical expansion stress, next to Donnan osmosis to account for tissue swelling.

The CSC experiment is a valuable instrument to determine mechanical properties of cartilaginous tissues, because of the well controlled loads and sample geometry. However, long experimentation times lead to PG loss which limits the validity of the experiments. Taking PG loss into account in the model overcomes this problem in part. CSC experiments can be used for the estimation of in vivo chemo-mechanical parameters of cartilaginous tissues.

\subsubsection{Recommendations}

Extra- and intrafibrillar fluid compartments; activity and osmotic coefficients The division of the fluid into extra- and intrafibrillar compartments requires quantification of additional activity and osmotic coefficients. These coefficients need to be determined, separately for extra- and intrafibrillar compartments, as a function of the size of the extrafibrillar compartment, the FCD, and the external ions concentrations. Thus, a relationship between the collagen content, FCD and external ions concentration, and the amount of fluid in the extrafibrillar compartment has to be determined. A first approximation to the intrafibrillar compartment with respect to activity and osmotic coefficients is to treat it as the external solution. The Poisson-Boltzmann model can be used to give estimates of the activity and osmotic coefficients for the Donnan model. Finally, quadriphasic theory and two-fluid-compartments theory should be merged into one model.

The computer fit of the swelling and compression stages of the CSC experiments with a single fluid compartment The existing triphasic model with Donnan osmosis needs to be improved, so that it is capable of predicting quantitatively the swelling properties of cartilaginous tissues with a realistic diffusion coefficient. The introduction of the electrochemical potential for the cations and anions separately (quadriphasic theory), together with the definition of two fluid compartments are possible improvements of the computer model.

The CSC setup and measurements; in vivo parameters The results of in vivo values of chemo-mechanical parameters of IVD tissue should be used for the assessment of chemo-mechanical models of cartilaginous tissues. The protocol given in chapter 5 can be used as a basis for this assessment. In order to determine in vivo parameters from CSC experiments it is advised to speed up the starting procedure and start the measurement of sample height before the sample has thawed.

Because of the existence of electrical potentials (Donnan, streaming, and diffusional potentials) in the mixture during experiments, one should be aware of the influence the environment may have on these potentials, and thus on the fluid flow and ion diffusion across the boundaries of the sample. Using electrically conducting surfaces of the walls and piston in a CSC setup may well influence the mechanical behaviour of the sample, and cause the boundary conditions to be essentially non-one-dimensional. It may therefore be better to use electrically non-conducting materials to construct a CSC setup. 
Knowledge of the effective (solid) stress, and stress free height can ultimately enable the researcher to predict situations in which solid failure can occur. The in vivo values of the parameters are useful to aid the designer of artificial materials that mimic the mechanical properties of the real biological materials, e.g., an artificial disc.

\subsection{Dependence on radial position of chemo-mechanic parameters and properties of the annulus fibro- sus of the canine intervertebral disc}

Measurements of various chemo-mechanical quantities have revealed radial dependencies in the annulus fibrosus. Dependencies of FCD, hydroxyproline, and fluid fraction are more or less corroborated by literature data. The increase of the bi- and triphasic permeability coefficients from outer to inner annulus, however, is not in accordance with any other experimental study.

\subsubsection{Fixed charge density, hydroxyproline, and fluid fraction}

For the FCD an increase was found from outer to inner annulus on dry weight basis, on wet weight basis, and on fluid volume basis $\left(c_{d w}^{p g}, c_{w w, v}^{p g}, c_{v}^{p g}\right.$; sections 5.4.1, 5.5.1). For the FCD on wet weight basis (human material) this finding is corroborated by Urban and Maroudas. ${ }^{219}$ For hydroxyproline (sections $5.4 .1,5.5 .1$ ) there is no general agreement. We found a decrease of the hydroxyproline content on dry weight basis (hypdw) from outer to inner annulus, but not on wet weight basis $\left(h y p_{w w, v}\right)$. Best et al. ${ }^{17}$ found for human discs a decrease of $h y p_{w w, v}$ from outer to inner annulus. Skaggs et al., ${ }^{199}$ also for human discs, found no correlation with radial position for hyp $p_{d w}$. Although there is general consent on the decrease of fluid fraction (sections 5.4.1,5.5.1) from the outer annulus to the inner (e.g. for human discs, Urban and Maroudas ${ }^{219}$ ), we did not find such a result for pooled samples from different discs and regions. However, for neighbouring samples from the same disc, we did find a higher fluid fraction for the inner sample for 4 out of 5 pairs.

\subsubsection{Permeability coefficient}

When the permeability coefficient of a porous material is discussed, it is very important to define exactly what permeability coefficient is meant, and how it was measured. In this thesis we have defined several permeability coefficients (section 3.3.1). From fits of time-displacement data during the compression stage of CSC experiments we determined biphasic (chapters 4 and 5 ) and triphasic permeability coefficients (sections 3.3.1, 5.3.4). The biphasic permeability coefficient, $K_{b i}$, is defined in an analytical solution of the compression of a porous medium consisting of a fluid and a solid phase (section 4.3.4). It relates fluid flow to the gradient in hydrostatic fluid pressure. For the analytical solution an infinitesimal strain $(<1 \%)$ is assumed. Furthermore, $K_{b i}$ is assumed constant.

In the CSC experiment, neither of these assumptions is satisfied. The strains are in the order of $10 \%$. Because $\bar{K}_{b i}$ depends on the pore size, and the pore size depends on the deformation, which is considerable, $K_{b i}$ is actually not a constant. The values found for $K_{b i}$ are therefore dependent on the deformational state of the sample before the testing. 
The triphasic permeability coefficient, $K_{t r i}$, relates fluid flow to the gradient of fluid pressure minus osmotic pressure. In contrast to the constant $K_{b i}$ defined in chapter 4 , it is chosen deformation dependent: every deformational state is identified with a unique $K_{\text {tri }}$.

For the biphasic permeability coefficient that was determined from the transients going from the conditioning (section 4.3.4), or swelling (section 5.3.4) equilibrium to the compression equilibrium, a dependence on the radial position of the sample in the disc was found (section 4.4, Figure 4.4, section 5.4, Figure 5.2b): $K_{b i}$ was higher for more inward annulus samples. ${ }^{b}$ However, $K_{b i}$ also increased as a function of the strain of the conditioning phase, $\epsilon_{\text {cond }}$ (section 4.4, Figure 4.5). The following question arises: could the increase of $K_{b i}$ with distance to the annulus edge after conditioning be caused by the conditioning of the sample? In other words: is there still a radial dependence of $K_{b i}$ before conditioning, i.e., in the in vivo configuration of the sample? It was found that conditioning the sample was indeed at least in part responsible for the decrease of $K_{b i}$ of outer samples (section 4.4, Figure 4.5). Bilinear regression with the distance $d$ of the mid-sample to the annulus edge, and the conditioning strain, $\epsilon_{\text {cond }}$ (section 4.4) revealed that $K_{b i}$ increased significantly with both $d$ and $\epsilon_{c o n d}$. The conclusion therefore is that the biphasic permeability in the in vivo state increases with distance from the annulus edge.

For the triphasic permeability coefficients, $K_{\text {tri }}$, determined from the transient of the swelling to the compression equilibrium by means of a computer fit (sections 5.3.4, 5.4.5), a similar radial dependence was found for the $K_{t r i}$ of the swelling and compression equilibria (section 5.4.5, Figure 5.6a). Extrapolation to the in vivo situation also showed a significantly increasing $K_{t r i, v}$ (section 5.4.5, Figure 5.6b).

The results of both bi- and triphasic permeability coefficients thus strongly suggest that the inner annulus is more permeable than the outer for fluid flow in the in vivo situation.

\subsubsection{Conclusions}

A radial dependence of FCD, hydroxyproline, and permeability coefficients was found for pooled samples of different levels and regions. For FCD, the increase with distance from annulus edge, was corroborated by results for human material. For hydroxyproline, decrease with distance to the annulus edge was found on dry weight basis, but not on wet weight basis. There was no general agreement of findings in the literature. The fluid content for pooled samples showed no correlation with distance to the annulus edge. However, for 4 out of 5 pairs of neighbouring samples, the inner sample had a higher fraction of fluid. The bi- and triphasic permeability coefficients increased from outer to inner annulus, which had not been found previously.

\footnotetext{
${ }^{b}$ Note that the conditioning equilibrium conditions used in the experiments of chapter $4(0.0772$, or $0.0778 \mathrm{MPa}, 0.16 \pm 0.1 \mathrm{M}$ ), equal the swelling equilibriurn conditions of chapter $5(0.0772$, or 0.0778 $\mathrm{MPa}, 0.159 \pm 0.007 \mathrm{M}$ ). The compression equilibrium conditions for both series of experiments are also equal $(0.194 \mathrm{MPa}, 0.16 \mathrm{M})$.
} 


\subsection{Relevance of this study and future use}

This study represents a step towards a better understanding of the chemo-mechanical behaviour of cartilaginous tissues, specifically the intervertebral disc annulus fibrosus. The intervertebral disc (IVD) is an important mechanical sub-unit of the spine, making possible complex movements of the back. The processes that affect the disc in normal aging and abnormal use, or abuse (traumas) are at present not completely understood. In order to understand what processes are of importance in the mechanical functioning of the disc, a physically realistic model is needed. The triphasic model used in this thesis is an important step towards such a model. It comprises a separation of elastic, osmotic, and viscous forces.

Why is it so important to use a physically realistic model for the intervertebral disc? Why would a phenomenological model not suffice? A phenomenological model might be capable of modeling mechanical behaviour, but does not elucidate the underlying physical principles. An example of a phenomenological model is a spring-damper model. The elastic force of the solid, together with the osmotic swelling force, are represented in the spring. The viscosity of the material is represented in the damper. The osmotic swelling pressure depends on the concentration of the external salt, and the fixed charge density. The spring-damper model is not able to predict concentration-dependent swelling phenomena, which are important for the disc in maintaining its integrity. E.g., swelling plays a role in the diurnal variation of the disc's height. A spring-damper model would neither be able to predict the consequences of aging, because these comprise changes in the water and proteoglycan content, which influences the osmotic pressure. Possibly also changes in the stiffness of the collagen fibres in the disc are involved.

Another example is the matter of traumas to the disc. In, e.g., a disc hernia, it is the solid fibre that fails under a certain combination of stress and strain. It is possible, that the rate of change of the fibre strain is an important factor in the damaging process. Thus, a solid component is needed to gain insight in the process of disc hernia. Another important factor that influences the choice of model is the desire to make synthetic discs that can replace the IVD in case of dysfunctioning, or after a trauma. In order to mimic the IVD's properties, it is imperative to have a physically realistic model.

Where does this study stand in the light of the requirements above? This study is still at a pretty fundamental stage, in the process of unraveling the mechanical secrecies of the intervertebral disc. The study has focused on the determination of the permeability coefficient for radial fluid flow, and the estimation of elastic, viscous, and osmotic forces that play a role in IVD mechanics. The permeability coefficient is an important parameter for the mechanics of the IVD, as it determines the rate of fluid flow through the disc. Because the disc is an avascular structure, nutrition of the disc, and the removal of waste products out of the disc, depend on the permeability. The matter of radial variation in disc permeability (chapters 4 and 5), which has not been found previously, will give reason for discussion on the interpretation of the results, and the experimental techniques used.

This study is also the first to present a protocol for determination of chemo-mechanical parameters of the IVD annulus based on a poro-elastic view with Donnan osmosis (triphasic model) in combination with a model for the separation of the fluid into an extra- and intrafibrillar compartment. This combination of models improves the quantification of 
the osmotic force, and thus also the elastic and viscous forces.

The triphasic (Donnan osmosis) model has been proven to be insufficient in the prediction of swelling transient behaviour of annulus fibrosus (although it works satisfactorily for equilibria and compression transients). For swelling due to changes in external jons concentrations, a transition to the quadriphasic model (possibly in combination with extra- and intrafibrillar fluid compartments) seems necessary. The quadriphasic model is able to model the effects of electrical potentials by using the electrochemical potentials of the separate ions. Electrical potentials are present in any process involving separation and flow of electrical charges. The Donnan potential is present against any gradient of chemical potential of an ionic species; the streaming and diffusion potentials are generated during fluid flow and ion diffusion in cartilaginous tissues.

Besides the expected advantage in the mechanics modeling, there is another reason for introducing a model that incorporates electrical phenomena. Many biological processes are controlled by electrical potential differences. Nerves and muscles work on electricity, transports of small ions across cell membranes depend on the gradients of the electrochemical potential. During physiological functioning there is always fluid flow in the disc. Because of the gravity and muscle forces on the disc, the IVD is compressed during normal daily activity. During bed rest, the disc swells due to an osmotic swelling pressure that exceeds the mechanical pressure on the disc. Therefore, streaming and diffusion potentials are expected to be normally present in the disc. These potentials might have an influence on the membrane potentials of the cells in the tissue, and thus might play a role in the vital processes of these cells. These processes involve intake of nutrients, and production of new proteoglycan and collagen molecules. One might also speculate on the role that electrical phenomena play in the aging process. The aging process of an intervertebral disc is associated with a change of composition (less water and proteoglycans) and a subsequent change of mechanical properties of the disc. How do these changes come about, and is it possible that electrical potentials play a role in them? Although these aspects are outside the scope of this study, further research can be motivated to find answers to these questions.

One question the researcher should never stop asking himself: into what detail should one go in making models of the biological tissue for a functional understanding of the tissue. In other words: to what depths should an investigator go in unraveling the chemo(electro-)mechanical mysteries of tissues like the IVD, while still calling the research relevant for medical science? Where does relevance for medical purposes border on the satisfaction of the curiosity of the researcher? 


\section{References}

1. Acaroglu ER, Iatridis JC, Setton LA, Foster RJ, Mow VC, Weidenbaum M. Degeneration and aging affect the tensile behaviour of human lumbar anulus fibrosus. Spine 1995;20:2690-2701.

2. Adams MA, Dolan P, Hutton WC. Diurnal variations in the stresses on the lumbar spine. Spine 1987;12:130-173.

3. Adams MA, Dolan P, Hutton WC, Porter RW. Diurnal changes in spinal mechanics and their clinical significance. J Bone Joint Surg Br 1990;72:266-270.

4. Adams MA, Hutton WC. The effect of posture on the fluid content of intervertebral discs. Spine 1983;8:665-671.

5. Adams $\mathrm{P}$, Eyre DR, Muir $\mathrm{H}$. Biochemical aspects of development and ageing of human lumbar intervertebral discs. Rheumatol Rehabil 1977;16:22-.

6. Adams $\mathrm{P}, \mathrm{Muir} \mathrm{H}$. Qualitative changes with age of proteoglycans of human lumbar discs. Ann Rheum Dis 1976;35:289-296.

7. Alcalay M, Chartier I, Garrouste O, Roualdes G, De Cursay GL, Azais I, Debiais F, Bontoux D. [chemonucleolysis of disk herniation with low back pain as the single symptom: 20 cases]: Chimionucleolyse des hernies discales avec lombalgie pure: vingt cas. Rev Rhum Ed Fr 1994;61:839-844.

8. Anderson HC, Sajdera SW. The fine structure of bovine nasal cartilage: Extraction as a technique to study proteoglycans and collagen in cartilage matrix. J Cell Biol 1972;49:650-663.

9. Andersson GBJ, Oertengren R, Nachemson A. Intradiskal pressure, intra-abdominal pressure and myoelectric back muscle activity related to posture and loading. Clin Orthop 1977;129:156-164.

10. Aspden RM, Hickey DS, Hukins DWL. Determination of collagen fibril orientation in the cartilage of vertebral end plate. Connect Tissue Res 1981;9:83-.

11. Basser PJ, Grodzinsky AJ. The Donnan model derived from microstructure. Biophysical Chemistry 1993;46:57-68.

12. Bathe KJ. Finite element procedures in engineering analysis:first ed. Englewood Cliffs, New Jersey:Prentice Hall Inc:1982. ISBN: 0-13-317305-4.

13. Bayliss MT, Johnstone B, O'Brian JP. Proteoglycan synthesis in human intervertebral disc. variation with age, region and pathology. Spine 1988;13:972-981.

14. Belytschko T, Kulak RF, Schultz AB, Galante JO. Finite element stress analysis of an intervertebral disc. J Biomechanics 1972;7:277-285.

15. Bernick S, Walker JM, Paule WJ. Age changes to the anulus fibrosus in human intervertebral discs. Spine 1991;16:520-524. 
16. Berthet-Colominas C, Miller A, Herbage D, Ronziere MC, Tocchetti D. Structural studies of collagen fibres from intervertebral disc. Biochim Biophys Acta $1982 ; 706: 50-64$.

17. Best BA, Guilak F, Setton LA, Zu W, Saed-Nejad F, Ratcliffe A, Weidenbaum M, Mow VC. Compressive mechanical properties of the human anulus fibrosis and their relationship to biochemical composition. Spine 1994;19:212-221.

18. Biot MA. General theory of three dimensional consolidation. J App Phys $1941 ; 26: 155-164$.

19. Biot MA. Theory of elasticity and consolidation for a porous anisotropic solid. J App Phys 1955;26:182-185.

20. Bishop PB. Proteoglycans and degenerative spondylosis. J Manipulative Physiol Ther 1988;11:36-40.

21. Blumenkrantz N, Sylvest J, Asboe-Hansen G. Local low collagen content may allow herniation of intervertebral disc: biochemical studies. Biochem Med 1977;18:283290 .

22. Boos N, Wallin A, Schmucker T, Aebi M, Boesch C. Quantitative MR imaging of lumbar intervertebral disc and vertebral bodies: methodology, reproducibility, and preliminary results. Magn Res Imaging 1994;12:577-587.

23. Borenstein D. Low back pain: epidemiology, etiology, diagnostic evaluation, and therapy. Curr Opin Rheumatol 1991;3:207-217.

24. Bowen RM. Toward a thermodynamics and mechanics of mixtures. Arch Rat Mech Ana! 1967;24:370-403.

25. Bowen RM. Incompressible porous media models by use of the theory of mixtures. Int J Engng Sci 1980;18:1129-1148.

26. Brickley-Parsons D, Glimcher MJ. Is the chemistry of collagen in intervertebral discs an expression of Wolff's law? Spine 1984;9:148-163.

27. Broberg KB. Slow deformation of intervertebral discs. J Biomechanics 1993;26:501512.

28. Buckwalter JA. The fine structure of human intervertbral disc. In: A A White, M M Panjabi, eds. Clinical biomechanics of the spine. 1st ed. Philadelphia, Toronto: J B Lippincott Co.:1978:ch. 9, pp. 108-143.

29. Buckwalter JA, Cooper RR, Maynard JA. Elastic fibres in human intervertebral discs. J Bone and Joint Surgery 1976;58-A:73-76.

30. Buckwalter JA, Cooper RR, Maynard JA. Ultrastructure of human intervertebral disc-new findings. Trans Ortho Res Soc 1976;1:130- 
31. Buckwalter JA, Pedrini-Mille A, Pedrini V, Tudisco C. Proteoglycans of human infant intervertebral disc. Electron microscopic and biochemical studies. J Bone Joint Surg Am 1985;67:284-294.

32. Buckwalter JA, Roughley PJ, Rosenberg LC. Age-related changes in cartilage proteoglycans: quantitative electron microscopic studies. Microsc Res Tech $1994 ; 28: 398-408$.

33. Buschmann MD, Grodzinsky AJ. A molecular model of proteoglycan-associated electrostatic forces in cartilage mechanics. J Biomech Eng 1995;117:179-192.

34. Bushell GR, Ghosh P, Taylor TKF, Akeson WH. Proteglycan chemisrtry of the intervertebral discs. Clin Orthop Relat Res 1977;129:115-.

35. Carlslaw HS, Jaeger JC. Conduction of heat in solids:first ed. Oxford, UK:Clarendon Press:1974.

36. Cassisi JE, Sypert GW, Lagana L, Friedman EM, Robinson ME. Pain, disability, and psychological functioning in chronic low back pain subgroups: myofascial versus herniated disc syndrome. Neurosurgery 1993;33:379-385.

37. Center for Health Statistics N. Limitation of activity due to chronic conditions. United States, 1969 and 1970, Series 10, No. $801973 ;: 277-285$.

38. Chang R. Physical chemistry with applications to biological systems:2 ed. New York:MacMillan Publishing Co., Inc.:1981. ISBN: 0-02-979050-X.

39. Chiu KY, Luk KD. Cord compression caused by multiple disc herniations and intraspinal cyst in scheuermann's disease. Spine 1995;20:1075-1079.

40. Cole TC, Burkhardt D, Frost L, Ghosh P. The proteoglycans of the canine intervertebral disc. Biochim Biophys Acta 1985;839:127-138.

41. Cole TC, Ghosh P, Taylor TKF. Variations of the proteoglycans if the canine intervertebral disc with ageing. Biochim Biophys Acta 1986;880:209-219.

42. Comper WD, Laurent TC. Physiological function of connective tissue polysacharides. Physiological Reviews 1978;58:255-315.

43. Cooper RG, Freemont AJ, Hoyland JA, Jenkins JP, West CG, Illingworth KJ, Jayson MI. Herniated intervertebral disc-associated periradicular fibrosis and vascular abnormalities occur without inflammatory cell infiltration. Spine 1995;20:591-598.

44. Cooper RR, Misol S. Tendon and ligament insertion: A light and electron microscopic study. J Bone and Joint Surgery 1970;52-A:1-21.

45. Creemers MR, Leijendeckers PHH, van Maarschalkerwaart MCM, Rijnsdorp JE, Tysma S. Polytechnisch zakboekje:40 ed. Arnhem, the Netheriands:Koninklijke PBNA:1983. 
46. Crock HV, Goldwasser M. Anatomic studies of the circulation in the region of the intervertebral end-plate in adult greyhound dog. Spine 1984;9.

47. Crock HV, Goldwasser M, Yoshizawa H. Vascular anatomy related to the intervertebral disc. In: P Ghosh, ed. The biology of the intervertebral disc, vol. 1. 1st ed. Boca Raton, Florida: CRC Press Inc.:1988:ch. 4, pp. 109-133.

48. De Palma AF, Rothman RH. The intervertebral disc. In: Philadelphia: W.B. Saunders Co.:1970:pp. 26-30.

49. Dickson IR, Happey F, Pearson CH, Naylor A, Turner RL. Variation in protein components of the human intervertebral disc with age. Nature 1967;215:52-53.

50. Donnan FG. The theory of membrane equilibria. Chemical Review 1924;1:73-90.

51. Drost MR, Willems P, Snijders H, Huyghe JM, Janssen JD, Huson A. Confined compression of canine annulus fibrosus under chemical and mechanical loading. J Biomech Eng 1995;117:390-396.

52. Eisenberg SR, Grodzinsky AJ. Swelling of articular cartilage and other connective tissues: electromechanical forces. J Orthop Res 1985;3:148-159.

53. Eisenberg SR, Grodzinsky AJ. The kinetics of chemically induced nonequilibrium swelling of articular cartilage and and corneal stroma. J Biomech Eng 1987;109:7989.

54. Eyre DR. Biochemistry of the intervertebral disc. Int Rev Connect Tissue Res $1979 ; 8: 227-291$.

55. Eyre DR. Collagens of the disc. In: P Ghosh, ed. The biology of the intervertebral disc, vol. 1. 1st ed. Boca Raton, Florida: CRC Press Inc.:1988:ch. 7, pp. 171-188.

56. Eyre DR, Muir H. Types I and II collagen in intervertebral disc. Biochem J 1976;157:267-.

57. Eyre DR, Muir H. Quantitative analysis of types I and II collagens in intervertebral disc at various ages. Biochim Biophys Acta 1977;492:29-.

58. Eyring EJ. The biochemistry and physiology of the intervertebral disc. Clin Orthop $1969 ; 67: 16-28$.

59. Freeman WDSC, Maroudas A. Charged group behaviour in cartilage proteoglycans in relation to ph. Ann Rheum Dis 1975;34, Suppl 2:44-.

60. Fung YC. Biomechanics, mechanical properties of living tissue:1 ed. New York:Springer-Verlag:1981.

61. Galanta JO. Tensile properties of the human lumbar annulus fibrosus. Acta Orthop Scan Suppl 1967;100:5-91.

62. Ghosh P, ed. The biology of the intervertebral disc. Boca Raton, Florida:CRC Press, Inc:1988. 2 volumes: vol. 1: ISBN 0-8493-6711-5, vol. 2: ISBN 0-8493-6712-3. 
63. Ghosh P, Bushell GR, Taylor TKF, Akeson WK. Collagens, elastin and noncollagenous protein of the intervertebral disc. Clin Orthop Relat Res 1977;1:9:124-.

64. Ghosh P, Taylor TK, Braund KG, Larsen LH. The collagenous and non-collagenous protein of the canine intervertebral disc and their variation with age, spinal level and breed. Gerontology 1976;22:124-134.

65. Goel VK, Monroe BT, Gilbertson LG, Brinckmann P. Interlaminar shear stresses and laminae separation in a disc. Finite element analysis of the 13-14 motion segment subjected to axial compressive loads. Spine 1995;20:689-698.

66. Gower WE. Pedrini V. Ag'-related variations in protein-polysaccharide from human nucleus pulposus, annulus fibrosus and costal cartilage. J Bone and Joint Surgery 1969;51A:1154-.

67. Graven R. Meting van radiale uitbolling aan een tussenwervelschijf. Tech. Rep. WFW 93.146:Eindhoven University of Technology, Department of Mechanical Engineering:Eindhoven, The Netherlands:1993. Measurement of the radial bulge of an intervertebral disc: in Dutch.

68. (irodzinsky AJ. Electromechanical and physicochemical properties of connective tissue. CRC critical reviews in biomedical engineering 1983;9:133-199.

69. (Grodzinsky AJ, Roth V, Myers E, Grossman WK, Mow VC. The significance of electromechanical and osmotic forces in the nonequilibrium swelling behavior of articular cartilage in tension. J Biomech Eng 1981;103:221-231.

70. ( ' W WY, Lai WM, Mow VC. Transport of fluid and ions 1.brough a porous-permeable charged-hydrated tissue, and streaming potential data on normal bovine articular cartilage. J Biomechanics 1993;26:709-723.

71. Gu WY, Lai WM, Mow VC. Transport of fluid and ions through a porous-permeable charged-hydrated tissue, and streaming potential data on normal bovine articular cartilage. J Biomechanics 1993;26:709-723.

72. Hascall VC, Hascall GK. Proteoglycans. In: E D Hay, ed. Cell biology of extracellular matrix, 1st ed. Plenum:1983:pp. 39-60.

73. Hayes WC, Keer LM, Hermann G, Mockros LF. A mathematical analysis for indentation tests of articular cartilage. J Biomechanics 1972;5:541-551.

74. Helfferich F. Ion exchange:1 ed. McGraw-Hill:1962.

75. Hickey DS, Hukins DW. Collagen fibril diameters and elastic fibres in the annulus fibrosus of human fetal intervertebral disc. J Anat 1981:133:351 -.

76. Hickey DS, Hukins DW. Aging changes in the macromolecular organization of the intervertebral disc: an $x$-ray diffraction and electron microscopic study. Spine $1982 ; 7: 234-242$. 
77. Hickey DS, Hukins DWL. Effect of methods of preservation on the arrangement of collagen fibrils in connective tissue matrices: an $\mathrm{x}$-ray diffraction study on annulus fibrosis. Conn Tissue Res 1979;6:223-228.

78. Hickey DS, Hukins DWL. Relation between the structure of the anulus fibrosus and the function and failure of the intervertebral disc. Spine 1979;5:106-116.

79. Hickey DS, Hukins DWL. X-ray diffraction studies of the arrangement of collagenous fibres in human fetal intervertebral disc. J Anat 1980;131:81-90.

80. Holmes MH. Finite deformation of soft tissue: analysis of a mixture model in uniaxial compression. J Biomech Eng 1986;108:372-381.

81. Hoimes MH, Lai WM, Mow VC. Singular perturbation analysis of the nonlinear, flow-dependent compressive stress relaxation behavior of articular cartilage. $\mathrm{J}$ Biomech Eng 1985;107:206-218.

82. Hormel SE, Eyre DR. Collagen in the ageing human intervertebral disc: an increase in covalently bound fluophores and chromophores. Biochim Biophys Acta 1991;1078:243-250.

83. Houben GB, Drost MR, Huyghe JM, Janssen JD, Huson A. Non-homogeneous permeability of canine anulus fibrosus. Spine 1997; Publication in January 1997.

84. Hukins DWL. Disc structure and function. In: P Ghosh, ed. The biology of the intervertebral disc, vol. 1. 1st ed. Boca Raton, Florida: CRC Press Inc.:1988:ch. 1, pp. 1-37.

85. Humzah MD, Soames RW. Human intervertebral disc: structure and funcion. The Anat Record 1988;220:337-356.

86. Inoue $\mathrm{H}$. Three-dimensional architecture of lumbar intervertebral discs. Spine 1981;6:139-

87. Johnson EF, Berryman H, Mitchell R, Wood WB. Elastic fibres in the annulus fibrosus of adult human lumbar intervertebral disc. A preliminary report. J Anat $1985 ; 143: 57-63$.

88. Johnson EF, Caldwell RW, Berryman HE, Miller A, Chetty K. Elastic fibres in the annulus fibrosus of the dog intervertebral disc. Acta Anat 1984;118:238-.

89. Johnstone B, Bayliss MT. The large proteoglycans of the human intervertebral disc. changes in their biosynthesis and structure with age, topography and pathology. Spine 1995;20:674-684.

90. Jonsson $\mathrm{B}$, Stromqvist $\mathrm{B}$. The straight leg raising test and the severity of symptoms in lumbar disc herniation. A preoperative evaluation. Spine 1995;20:27-30.

91. Kaapa E, Han X, Holm S, Peltonen J, Takala T, Vanharanta H. Collagen synthesis and types I, III, IV, and VI collagens in and animal model of disc degeneration. Spine 1995;20:59-66. 
92. Katchalsky A, Curran PF. Nonequilibrium thermodynamics in biophysics:2 ed. Cambridge, Massachusetts:Harvard University Press:1967.

93. Katz EP, Wachtel EJ, Maroudas A. Extrafibrillar proteoglycans osmotically regulate the molecular packing of collagen in cartilage. Biochim Biophys Acta 1986;882:136139.

94. Kazarian L. Creep characteristirs of the human spinal column. Orth Clin North Am 1975;6:3-18.

95. Kazarian L. Injuries to the human spinal column: biomechanics and infjury classification. Exerc Sport Sci Rev 1981;9:297-352.

96. Keith DA, Paz MA, Gallop PM, Glimcher MJ. Histologic and biochemical identification and characterization of elastin in cartilage. J Histochem Cytochem $1977 ; 25: 1154-1162$.

97. Keller TS, Hansson TH, Holm SH, Pope MM, Spengler DM. In vivo creep behaviour of the normal and degenerated porcine intervertebral disc: a preliminary report. J Spinal Disord 1988;1:267 278.

98. Keller TS, Holm SH, Hansson TH, Spengler DM. The dependence of intervertebral disc mechanical properties on physiologic conditions. Spine 1990;15:751-761. 1990 Volvo award in experimental studies.

99. Keller TS, Spengler DM, Hansson TH. Mechanical behaviour of the human lumbar spine. I. Creep analysis during static compressive loading. J Orthop Res 1987;5:46478.

100. Kelsey JL. Ideopathic low back pain. Magnitude of the problem. In: A A White III, S L Gordon, eds. Symposium on low back pain. St Louis - Toronto - London: The C V Mosby Company:1982:ch. 1, pp. 5-8.

101. Kitano T, Zerwekh JE, Usui Y, Edwards ML, Flicker PL, Mooney V. Biochemical changes associated with the symptomatic human intervertebral disc. Clin Orthop $1993 ; 293: 373-377$.

102. Koeller W, Funke F, Hartman F. Biomechanical behaviour of human intervertebral discs subjected to long lasting axial loading. Biorheology 1984;21:675-686.

103. Kraemer J, Kolditz D, Gowin R. Water and electrolyte content of human ivd under variable load. Spine 1983;10:69-71.

104. Krajickova J, Polakova R, Smetana K, Vytasec R. Age-dependent changes in proteoglycan biosynthesis in human intervertebral dises. Folia Biol Praha 1995;41:41-51.

105. Kurowski $\mathrm{P}$, Kubo A. The relationship of degeneration of the intervertebral disc to mechanical loading conditions on lumbar vertebrae. Spine 1986;11:726-731. 
106. Kwak JCT. Mean activity coefficients for the simple electrolyte in aequeous mixtures of polyelectrolytes and simple electrolytes. the system sodium polystyrenesulfonatesodium chloride. J Phys Chem 1973;77:2790-3793.

107. Kwan MK, Hacker SA, Woo SLY, Wayne JS. The effect of storage on the biomechanical behaviour of articular cartilage - a large strain study. J Biomech Eng 1992;114:149-153.

108. Kwan MK, Lai WM, Mow VC. A finite deformation theory for cartilage and other soft hydrated connective tissues. J Biomechanics 1990;23:145-155.

109. Lai WM, Hou JS, Mow VC. A triphasic theory for the swelling and deformation behaviors of articular cartilage. J Biomech Eng 1991;113:245-258.

110. Lai WM, Mow VC, Roth V. Effects of non-linear strain dependent permeability and rate of compression on the stress behavior of articular cartilage. J Biomech Eng $1981 ; 103: 61-66$.

111. Laible JB, Pflaster DS, Krag MH, Simon BR, Haugh LD. A poroelastic-swelling finite element model with application to the intervertebral disc. Spine 1993;18:659670 .

112. Lanir Y. Biorheology and fluid flux in swelling tissues. I Bicomponent theory for small deformations, including concentration effects. Biorheology 1987;24:173 187.

113. Lanir Y. Biorheology and fluid flux in swelling tissues. II Analysis of unconfined compressive response of transversely isotropic cartilage disc. Biorheology 1987;24:189205.

114. Lawrence JS. Rheumatism in populations: 1 ed. London:William Heinemann Medical Books Ltd.:1977.

115. Li ST, Katz EP. Electrostatic properties of reconstituted collagen fibrils. In: C T Brighton, J Black, S R Pollack, eds. Electrical properties of bone and cartilage. 1st ed. New York: Grune and Stratton:1979:pp. 119-.

116. Lim TH, Goel VK, Weinstein JN, Kong W. Stress analysis of a canine spinal motion segment using the finite element technique. J Biomechanics 1994;27:1259-1269.

117. Lipson SJ, Muir H. Experimental intervertebral disc degeneration: morphologic and proteoglycan changes over time. Arthritis Rheum 1981;24:12-21.

118. Lyons G, Eisenstein SM, Sweet MB. Biochemical changes in intervertebral disc degeneration. Biochim Biophys Acta 1981;673:443 4;3.

119. Mackie JS, Meares P. Diffusion of electrolyt $\cdot$ in cation exchange resin. Proc $K$ Soc London Ser A 1955:232:498.

120. Mak AF. The apparent viscoelastic behaviour of articular cartilage- The contributions from the intrinsic matrix viscoelasticity and interstitial fluid flows. J Biomech Eng 1980;108:123-130. 
121. Manning GS. Limiting laws and counterion condensation in polyelectrolyte solutions. J Chem Physics 1969;51:924-.

122. Mansour JM, Mow VC. The permeability of articular cartilage under compressive strain and high pressures. J Bone and Joint Surgery 1976;58-A:509-516.

123. Marchand F, Ahmed AM. Mechanical properties and pressure mechanisms of the lumbar disc anulus. Trans Ortho Res Soc 1989;14:355.

124. Marchand F, Ahmed AM. Investigation of the laminate structure of lumbar disc anulus fibrosus. Spine 1990;15:402-410.

125. Maroudas A. Physicochemical properties of cartilage in the light of ion exchange theory. Biophys J 1968;8:575-595.

126. Maroudas A. Biophysical chemistry of cartilaginous tissues with special reference to solute and fluid transport. Biorheology 1975;12:233-248.

127. Maroudas A. Fluid transport in cartilage. Ann Rheum Dis Suppl 1975;34:77-81.

128. Maroudas A. Physicochemical properties of articular cartilage. In: M A R Freeman, ed. Adult articular cartilage. 2nd ed. Tunbridge Wells, Kent, UK: Pitman medical:1979:ch. 4, pp. 215-290.

129. Maroudas A. Nutrition and metabolism of the intervertebral disc. In: $P$ Ghosh, ed. The biology of the intervertebral disc, vol. 1. 1st ed. Boca Raton, Florida: CRC Press Inc.:1988:ch. 9, pp. 1-38.

130. Maroudas A, Bannon C. Measurement of swelling pressure in cartilage and comparison with the osmotic pressure of constituent proteoglycans. Biorheology $1981 ; 18: 619-632$.

131. Maroudas A, Evans H. A study of ionic equilibria in cartlage. Connect Tissue Res $1972 ; 1: 69-77$.

132. Maroudas A, Stockwell RA, Nachemson A, Urban J. Factors involved in the nutrition of the human lumbar intervertebral disc: cellularity and diffusion of glucose in vitro. J Anat 1975;120:113-130.

133. Maroudas A, Thomas H. A simple physicochemical micromethod for determining fixed anionic groups in connective tissue. Biochim Biophys Acta 1970;215:214-216.

134. Maroudas A, Urban J. In vitro methods for studying articular cartilage and intervertebral disc. In: A S Kunin, D J Simmons, eds. Skeletal research: an experimental approach, vol. 2. 1st ed. London: Academic Press Inc.:1983:ch. 6, pp. 135-182.

135. Maroudas A, Wachtel E, Grushko G, Katz EP, Weinberg P. The effect of osmotic and mechanical pressures on water partitioning in articular cartilage. Biochim Biophys Acta $1991 ; 1073: 285-294$. 
136. Maroudas A, Ziv I, Weisman N, Venn M. Studies of hydration and swelling pressure in normal and osteoarthritic cartilage. Biorheology 1985;22:159-169.

137. McGill CM. Industrial back problems. J Occup Med 1986;10:174-178.

138. McNally DS, Adams MA. Internal intervertebral disc mechanics as revealed by stress profilometry. Spine 1992;17:66-73.

139. Melrose J, Ghosh P. The noncollagenous proteins of the intervertebral disc. In: P Ghosh, ed. The biology of the intervertebral disc, vol. 1. 1st ed. Boca Raton, Florida: CRC Press Inc.:1988:ch. 8, pp. 189-237.

140. Melrose J, Ghosh P, Taylor TK, Hall A, Osti OL, Vernon-Roberts B, Fraser RD. A longitudinal study of the matrix changes induced in the intervertebral disc by surgical damage to the annulus fibrosus. J Orthop Res 1992;10:665-676.

141. Melrose J, Gurr KR, Cole TC, Darvodelsky A, Ghosh P, Taylor TK. The influence of scoliosis and ageing on proteoglycan heterogeneity in the human intervertebral disc. J Orthop Res 1991;9:68-77.

142. Mikhael MA, Paige ML, Widen AL. Magnetic resonance imaging and the diagnosis of avascular necrosis of the femoral head. Comput Radiol 1987;11:157-163.

143. Minns RJ, Soden PD, Jackson DS. The role of the fibrous components and ground substance in the mechanical properties of biological tissues: a preliminary investigation. J Biomechanics 1973;6:153-.

144. Mow VC, Holmes MH, Lai WM. Fluid transport and mechanical properties of articular cartilage. J Biomechanics 1984;17:377-394.

145. Mow VC, Kuei SC, Lai WM, Armstrong CG. Biphasic creep and stress relaxation of articular cartilage in compression: theory and experiments. J Biomech Eng $1980 ; 102: 73-84$.

146. Mow VC, Kwan MK, Lai WM, Holmes MH. A finite deformation theory for nonlinearly permeable cartilage and other soft hydrated connective tissues. In: S L-Y Woo, G Schmidd-Schonbein, B Zweifach, eds. Frontiers in biomechanics. 1st ed. New York: Springer-Verlag:1986:pp. 153-179.

147. Mow VC, Schoonbeck JM. Contribution of donnan osmotic pressure towards the biphasic compressive modulus of articular cartilage. Trans Ortho Res Soc $1984 ; 9: 262-$.

148. Muir H. Proteoglycans as organizers of the extracellular matrix. Biochem Soc Trans 1983;11:613-622.

149. Muir H, Bullough P, Maroudas A. The distribution of collagen in human articular cartilage with some of its physiological implications. J Bone and Joint Surgery 1970;52-B:554-563. 
150. Myers ER, Lai WM, Mow VC. A continuum theory and an experiment for the ioninduced swelling behavior of articular cartilage. J Biomech Eng 1984;106:151-158.

151. Nachemson AL. Disc pressure measurements. Spine 1981;6:93-97.

152. Nachemson AL, Lewin T, Maroudas A, Freeman MA. In vitro diffusion of dye through the end-plates and the annulus fibrosus of human lumbar inter-vertebral discs. Acta Orthop Scand 1970;41:589-607.

153. Nachemson AL, Schultz AB, Berkson MH. Mechanical properties of human lumbar spine motion segments: influence of age, sex, disc level and degeneration. Spine 1979;4:1-8.

154. Natali AN. A hyperelastic and almost incompressible material model as an approach to intervertebral disc analysis. J Biomech Eng 1991;13:163-168.

155. Natali AN, Meroi E. Cnonlinear analysis of intervertebral disk under dynamic load. J Biomech Eng 1990;112:358-363.

156. Naylor $\mathrm{A}$. The biochemical changes in the human intervertebtral disc in degeneration and nuclear prolapse. Orth Clin North Am 1971;2:343-358.

157. Oegema TR, Bradford DS, Cooper KM, Hunter RE. Comparison of the biochemistry of proteoglycans isolated from normal, ideopathic scoliotic and cerebral palsy spines. Spine 1983;8:378-384.

158. Ogata $\mathrm{K}$, Whiteside LA. Nutritional pathways of the intervertebral disc, an experimental study using hydrogen washout technique. Spine 1981;6:211-216. 1980 Volvo award winner in basic science.

159. Ohshima H, Tsuji H, Hirano N, Ishihara H, Katoh Y, Yamada H. Water diffusion pathway, swelling pressure, and biomechanical properties of the intervertebral disc during compression load. Spine 1989;14:1234-1244.

160. Olczyk K. Age-related changes in collagen of human intervertebral discs. Gerontology 1992;38:196-204.

161. Olczyk K. Age-related changes in glycosaminoglycans of human intervertebral discs. Folia Histochem Cytobiol 1994;31:215-220.

162. Olczyk K. Changes in macromolecular components of prolapsed intervertebral dises. Ann Biol Clin Paris 1994;52:711-716.

163. Oomens CWJ, de Heus HJ, Huyghe JM, Nelissen L, Janssen JD. Validation of triphasic mixture theory for a mimic of intervertebral disc tissue. Biomimetics 1996; Accepted.

164. Oomens CWJ, van Campen DH, Grootenboer HJ. A mixture approach to the mechanics of skin. J Biomechanics 1987;20:877-885. 
165. Osti OA, Vernon-Roberts B, Fraser RD. Anular tears and intervertebral disc degeneration. Spine 1990;15:762-767.

166. Overbeek JTG. The donnan equilibrium. Prog Biophys Biophys Chem 1956;6:58-84.

167. Panagiotacopulos ND, Knauss WG, Bloch R. On the mechanical properties of human intervertebral disc material. Biorheology 1979;16:317-330.

168. Panagiotacopulos ND, Pope MH, Bloch R, Krag MH. Water content in human intervertebral discs. Part I. measurement by magnetic resonance imaging. Spine 1987;12:912-917.

169. Panagiotacopulos ND, Pope MH, Bloch R, Krag MH. Water content in human intervertebral discs.Part II. Viscoelastic behaviour. Spine 1987;12:918-924.

170. Panjabi MM, Krag M, Summers D, Videman T. Biomechanical time-tolerance of fresh cadaveric human spine specimens. J Orthop Res 1985;3:292-300.

171. Pearson CH, Happey F, Naylor A, Osborn JM, Turner RL. Lysosomal enzymes and degradation of the proteoglycans of the human intervertebral disc. Biochem J $1972 ; 129: 44-$.

172. Piez KA. Primary structure. In: G N Ramachandran, A H Reddi, eds. Biochemistry of collagen. 1st ed. New York: Plenum Press:1976:ch. 1, pp. 1-.

173. Poole AR, Pidoux I, Reiner A, Rosenberg L. An immunoelectron microscope study of the organisation of the proteoglycan monomer, link protein, and collagen in the matrix of articular cartilage. J Cell Biol 1982;93:921-.

174. Preston BN, Snowdon JM, Houghton KT. Model connective tissue systems: the effect of proteoglycans on the distribution of small non-electrolytes and micro-ions. Biopolymers 1972;11:1645-1659.

175. Pueschell J. Der wassergehalt normaler und degenerierter zwischenwirbelscheiben. Beitr Path Anat 1930;84:123.

176. Pustaver MR. Mechanical low back pain: etiology and conservative management. J Manipulative Physiol Ther 1994;17:376-384.

177. Rabischong P, Louis R, Vergraud J, Masacre C. The intervertebral disc. Anat Clin $1978 ; 1: 5.5-64$.

178. Rao AA, Dumas GA. Influence of material properties on the mechanical behaviour of the $15-\mathrm{s} 1$ intervertebral disc in compression: a nonlinear finite element study. J Biomech Eng 1991;13:139-151.

179. Richards EG. An introduction to the physical properties of large molecules in solution:1 ed. Cambridge:Cambridge University Press:1980. ISBN: 0-521-23110-8.

180. Roberts S, Beard HK, O'Brian JP. Biochemical changes of intervertebral discs in patients with spondylolisthesis or with tears of the posterior annulus fibrosus. Ann Rheum Dis 1982;41:78-85. 
181. Roberts S, Caterson B, Evans H, Eisenstein SM. Proteoglycan components of the intervertebral disc and cartilage end plate: an immunolocalization study of animal and human tissues. Histochem $\mathrm{J}$ 1994;26:402-411.

182. Roberts S, Menage J, Eisenstein SM. The cartilage end-plate and intervertebral disc in scoliosis: calcification and other sequellae. J Orthop Res 1993;11:747-757.

183. Roberts S, Menage J, Urban JP. Biochemical and structural properties of the cartilage end-plate and its relation to the intervertebral disc. Spine 1989;14:166-174.

184. Robinson RA, Stokes RH. Electrolyte solutions. London, England:Butterworths:1968.

185. Scott JE, Bosworth TR, Cribb AM, Taylor JR. The chemical morphology of agerelated changes in human intervertebral disc glycosaminoglycans from cervical, thoracic, and lumbar nucleus pulposus and annulus fibrosus. J Anat 1994;184:73-82.

186. Shirazi-Adl A. On the fibre composite material models of disc annulus-comparison of predicted stresses. J Biomechanics 1989;22:357-365.

187. Shirazi-Adl A. Strain in fibers of a lumbar disc. analysis of the role of lifting in producing disc prolapse. Spine 1989;14:96-103.

188. Shirazi-Adl A. Finite-element simulation of changes in the fluid content of human lumbar discs. mechanical and clinical implications. Spine 1992;17:206-212.

189. Shirazi-Adl A. Biomechanics of the lumbar spine in sagittal/lateral moments. Spine 1994;19:2407-2414.

190. Shirazi-Adl A, Ahmed AM, Shrivastava SC. A finite element study of a lumbar motion segment subjected to pure sagittal plane moments. J Biomechanics 1986;19:331350 .

191. Shirazi-Adl A, Ahmed AM, Shrivastava SC. Mechanical response of a lumbar motion segment in axial torque alone and combined with compression. Spine 1986;11:914927.

192. Shirazi-Adl A, Shrivastava SC, Ahmed AM. Stress analysis of the lumbar discbody unit in compression. A three-dimensional nonlinear finite element study. Spine $1984 ; 9: 120-134$.

193. Silberberg A. The mechanics and thermodynamics of separation flow through porous, molecular disperse, solid media. The Poiseille Lecture. Biorheology 1982;19:111-127.

194. Silberberg A. Transport through deformable matrices. Biorheology 1989;26:291-313.

195. Simon BR, Gaballa MA. Poroelastic finite element models for the spinal motion segement including ionic swelling. In Computational methods in bioengineering (1988):ASME:pp. 93-99. 
196. Simon BR, Wu JS, Carlton MW, Evans JH, Kazarian LE. Structural models for human spinal motion segments based on a poroelastic view of the intervertebral disk. J Biomech Eng 1985;104:327-335.

197. Simon BR, Wu JS, Carlton MW, Kazarian LE, France EP, Evans JH, Zienkiewicz OC. Poroelastic dynamic structural models of rhesus spinal motion segments. Spine 1985;10:494-507.

198. Simon BR, Zienkievicz OC, Paul DK. Evaluation of $u-w$ and $u-\pi$ finite element models for the dynamic response of saturated porous media using one-dimensional models. Int J Num Ana Meth Geomech 1986;10:461-482.

199. Skaggs DL, Weidenbaum MD, Iatridis JC, Ratcliff A, Mow VC. Regional variation in tensile properties and biochemical composition of the human lumbar anulus fibrosus. Spine 1994;19:1310-1319.

200. Snijders H. The triphasic mechanics of the intervertebral disc. PhD dissertation:Limburg University:Department of Movement Sciences:1994.

201. Snijders H, Houben GB, Drost MR, Huyghe JM, Janssen JD, Huson A. Osmotic prestressing of a spinal motion segment. In Topics in applied mechanics (Dordrecht, The Netherlands, 1993):J. F. Dijksman and F. T. M. Nieuwstadt, Eds.:Kluwer Academic Publishers:pp. 321-330.

202. Snijders H, Huyghe JM, Janssen JD. A theory for the chemo-mechanical behaviour of annulus fibrosus tissue. Submitted:1994.

203. Snijders H, Huyghe JM, Janssen JD. Triphasic finite element model for swelling porous media. Int J Num Meth Fluids 1995;20:1039-1046.

204. Spilker RL, Daugirda DM, Schultz AB. Mechanical response of a simple finite element model of the intervertebral disc under complex loading. J Biomechanics $1984 ; 17: 103-112$.

205. Spilker RL, Jacobs DM, Schultz AB. Material constants for a finite element model of the intervertebral disk with a fiber composite annulus. J Biomech Eng 1986;108:111 .

206. Spilker RL, Maxian TA. A mixed-penalty finite element formulation of the linear biphasic theory of cartilaginous tissues. Int J Num Meth Eng 1990;30:1063-1082.

207. Stegemann H. Microbestimmung von hydroxyprolin mit chloramin-t und $\mathrm{p}$ - dimethylaminobenzaldehyd. Zeitschrift fuer Physiologische Chemie 1958;311:41-45.

208. Stokes IAF. Surface strain on human intervertebral discs. J Orthop Res 1987;5:348355.

209. Swezey RL. Pathophysiology and treatment of intervertebral disk disease. Rheum Dis Clin North Am 1993;19:741-758. 
210. Terzaghi K. Erdbaumechanik auf Bodenphysikalischen Grundlagen. Wien:Deuticke:1925.

211. Terzaghi KV. Die Berechnung des Durchlaessigkeitsziffer des Tones aus dem Verlauf der hydrodynamischen Spannungserscheinungen. Akademie der Wissenschaften in Wien. Matematisch naturwissenschapfliche Klasse 1923;123:125-138.

212. Timpl R, Wolff I, Weiser M. A new class of structural proteins from connective tissue. Biochim Biophys Acta 1968;168:168-.

213. Truesdell C, Toupin R. The classical field theories. In: S Fluegge, ed. Encyclopedia of physics III/1. Berlin: Springer:1960.

214. Ueno K, Liu YK. A three-dimensional nonlinear finite element model of lumbar intervertebral joint in torsion. J Biomech Eng 1987;109:200-209.

215. Urban JP, Holm S, Maroudas A, Nachemson A. Nutrition of the intervertebral disc: effect of fluid flow to solute transport. Clin Orthop 1982;170:296-302.

216. Urban JPG. Fluid and solute transport in the intervertebral disc. PhD dissertation:London University:London, England:1977.

217. Urban JPG, Holm S, Maroudas A. Diffusion of small solutes into the intervertebral disc: as in vivo study. Biorheology 1978;15:203-223.

218. Urban JPG, Holm S, Maroudas A, Nachemson A. Nutrition of the intervertebral disc. an in vivo study of solute transport. Clin Orthop 1977;129:101-114.

219. Urban JPG, Maroudas A. The measurement of fixed charge density in the intervertebral disc. Biochim Biophys Acta 1979;586:166-178.

220. Urban JPG, Maroudas A. Swelling of intervertebral disk in vivo. Connect Tissue Res 1979;9:1-10.

221. Urban JPG, Maroudas A. The chemistry of intervertebral disc in relation to its physiological function and requirements. Clin Rheum Dis 1980;9:1-10.

222. Urban JPG, Maroudas A. Measurement of swelling pressure and fluid flow in the intervertebral disc with reference to creep. J Mech Eng 1980;c132/80:63-69.

223. Urban JPG, Maroudas A, Bayliss MT, Dillon J. Swelling pressures of proteoglycans at the concentrations found in cartilaginous tissues. Biorheology 1979;16:447-464.

224. Urban JPG, McMullin JF. Swelling pressures of the intervertebral disc: influence of proteoglycan and collagen contents. Biorheology 1985;22:145-157.

225. Urban JPG, McMullin JF. Swelling pressures of the lumbar intervertebral discs: influence of age, spinal level, composition and degeneration. Spine 1988;13:179-187.

226. Valkenburg HA, Haanen HCM. The epidemiology of low back pain. In: A A White III, S L Gordon, eds. Symposium on low back pain. St Louis - Toronto - London: The C V Mosby Company:1982:ch. 2, pp. 9-22. 
227. Vasan NS, Gutteling EW, Lee CK, Cibischino M, Parsons JR. A preliminary study of mechanically stress-induced changes in the extracellular matrix of the canine intervertebral disc. Spine 1991;16:317-320.

228. Venn MF, Maroudas A. Chemical composition and swelling of normal and osteoarthritic femoral head cartilage. Ann Rheum Dis 1977;36:399-407.

229. Vermeer J. Klinische betekenis van degeneratieve afwijkingen van de lumbale wervelkolom. Verzekeringskundige aspecten bij de beoordeling van arbeidsongeschiktheid. Ned Tijdschr Geneesk 1983;12:1383-1438.

230. Virgin WJ. Experimental investigation into the physical properties of the intervertebral discs. J Bone and Joint Surgery 1951;33:607-611.

231. Wachtel E, Maroudas A. Characterization of the packing of collagen in cartilage using X-ray scattering. In: A Maroudas, K Kuettner, eds. Methods in cartilage research. 1st ed. London: Academic Press Inc.:1990:ch. 56, pp. 227-232.

232. Wachtel E, Maroudas A, Sclineiderman R. Age-related changes in collagen packing of human articular cartilage. Biochim Biophys Acta 1995;1243:239-243.

233. Wells JD. Salt activity and osmotic pressure in connective tissue. Proc R Soc Lond B 1973;183:399-.

234. White AA, Panjabi MM. Clinical biomechanics of the spine:first ed. PhiladelphiaToronto:J B Lippincott Company:1978. ISBN 379-50388-1.

235. Woltring HJ. A fortran package for generalised, cross validatory spline smoothing and differentiation. Adv Eng Software 1986;8:104-107.

236. Wu HC, Yao RF. Mechanical behaviour of the human anulus fibrosus. J Biomechanics 1976;9:1-7.

237. Wu JJ, Eyre DR, Slayter HS. Type VI collagen of the intervertebral disc. Biochemical and electron-microscopic characterization of the native protein. Biochem J 1987;284:373-381.

238. Zienkiewicz OC. The finite element method:third ed. Maidenhead, Berkshire, England:McGraw-Hill Ltd:1977. ISBN: 0-07-084072-5.

239. Zimmerman MC, Vuono-Hawkins M, Parsons JR, Carter FM, Gutteling E, Lee C, Langrana NA. The mechanical properties of the canine lumbar disc and motion segment. Spine 1992;17:213-220. 


\section{Summary}

The etiology of low back pain is often unclear. It is not always possible to link the occurrence of low back pain to abnormalities in the intervertebral disc. In the case of disc herniation sometimes a direct relationship with pain symptoms can be found. For an understanding of the factors that cause mechanical defects in the disc, micromodels including chemical and mechanical features of the intervertebral disc may be helpful. The intervertebral disc does not primarily sustain mechanical loads by means of elastic and viscous forces, but osmotic, or alternatively, electro-chemical forces fulfill an important role in the mechanical behaviour. For the understanding of the etiology of dise diseases, and the process of disc degeneration (aging), it is of vital importance to distinguish between the contributions of these three forces. In this way it is possible to make an accurate estimate of, e.g., the solid stress, which is most possibly of importance for the occurrence of disc hernia.

In the light of the requirements above, this study has focussed on the development of (1) a numerical model that accounts for elastic, osmotic, and viscous contributions to the mechanical functioning of intervertebral disc tissue, and (2) an experimental protocol separating these three contributions. The experimental protocol leads to quantitative estimates of model parameters, such as elastic stress, strain, activity and osmotic coefficients in the in vivo tissue. As such, it is a valuable new tool for assessment of mixture models of intervertebral disc.

The numerical model is based on mixture theory, and discriminates between three components (phases): solid, fluid, and salt molecules (ions). The choice of the degrees of freedom is based on the requirement that discontinuities in the ions or fixed charge concentrations do not lead to discontinuities in the degrees of freedom. As a result, the continuity of the degrees of freedom at the boundaries of the material is ensured.

The theory of the triphasic mixture model is described in chapter 2. The interaction between the three components is governed by the fundamental physics conservation laws. For the derivation of the differential equations, the first (conservation of energy) and second (increase of entropy) laws of thermodynamics are combined, where the increase of entropy is applied to the mixture as a whole, not on the components separately. Donnan osmosis is used as a model for material swelling. The basic assumptions for the mixture are as follows: the constituents are assumed to be intrinsically incompressible; the absolute temperature is assumed to be constant in time and place; the volume fraction of the ions is neglected compared to those of the solid and the fluid; mass transfer, and moment of momentum supply from one component to the other are neglected; inertia forces and body forces in the momentum equation are neglected.

Three coupled differential equations describing the motion of the solid, fluid and ions result from the triphasic model. These differential equations can not be solved analytically. Therefore, a finite element model is constructed. The degrees of freedom are the position vector of the solid, and the chemical potentials of the fluid and the salt molecules (chapter 3). For the simulation of one-dimensional confined swelling and compression experiments, a one-dimensional computer model has been developed. In a confined swelling and compression experiment, the height of a sample in time is measured as a function of the mechanical and chemical (osmotic) loads. The sample is confined in a cylinder, contacting a salt solution (chemical load) via a filter at the bottom, and mechanically 
loaded by means of a piston, placed on top of the sample. From the computer fits of the confined swelling and compression experiments, chemo-mechanical parameters, such as activity coefficients, permeability and diffusion coefficients, effective stress and stress free height, are determined.

The equilibrium situations and the compression transient of the experiments are fitted easily. However, it is not possible to fit the swelling transient concurrently with the compression transient. It appears that the diffusion coefficient is overestimated, whereas the rest of the parameter values is within the physiological range.

The radial variation of the permeability in the annulus fibrosus of the intervertebral disc has been investigated in chapter 4 . Fitting a biphasic analytical solution to the compression transient of a confined swelling and compression experiment, results in a biphasic permeability coefficient, and aggregate modulus. The biphasic permeability coefficient increases from outer to inner annulus. This result is corroborated by results from chapter 5. For the aggregate modulus as a function of radial position there is no conclusive finding: the aggregate modulus can either decrease (chapter 4), or increase (chapter 5) from outer to inner annulus. The biphasic permeability coefficient and aggregate modulus are deformation dependent.

In chapter 5 a series of experiments is designed to determine the in vivo chemomechanical properties of canine annulus fibrosus. The protocol for the determination of these properties is based on the division of tissue fluid into extra- and intrafibrillar water (two fluid compartments), and the use of Donnan osmotic theory for the determination of the osmotic pressure. The experiments used in the protocol are: one-dimensional confined swelling and compression experiments, measurement of fixed charges using radioactive labelling, measurements of hydroxyproline and fluid content. Research has revealed that part of the tissue water is bound to the collagen fibrils. This fraction is not available for the proteoglycans and is called the intrafibrillar fraction. The size (fluid content) of the intrafibrillar compartment is determined by the collagen (hydroxyproline) content and the osmotic pressures.

From the equilibrium situations of the confined swelling and compression experiments, a relationship between the effective stress (a measure for the solid stress), and the strain is determined. In vivo values of chemo-mechanical quantities, such as fixed charge density, fluid fraction, collagen content, osmotic and activity coefficients, effective stress and stress free length, are determined. Bi- and triphasic permeability coefficients are determined from fits of the compression transient, using the biphasic analytical solution (chapter 4), and the one-dimensional computer model of chapter 3 respectively. For the triphasic permeability coefficient also an estimate of the iñ vivo value is made. The triphasic computer model is also used for the determination of chemo-mechanical parameters based on a single fluid compartment, as was done in chapter 3 . The computer model, in contrast: to the analysis protocol for the equilibrium situations, does not discriminate between extra- and intrafibrillar water.

For the stress-strain relationship, we have chosen for a linear relationship between the effective 2nd Piola-Kirchhoff stress and the Green-Lagrange strain. This choice has important consequences for the stress and strain that are found for the in vivo situation. Using the linear relation, it is found that in the in vivo situation, the solid is under compression in the radial direction. The analysis based on extra- and intrafibrillar compartments yields different results from the single fluid compartment results of the 
computer simulation. The extent of the difference depends on the amount of water that is bound by the collagen fibrils. The analysis of the results give no indication for the introduction of chemical expansion stress to account for material swelling. The triphasic in vivo permeability coefficient also increases from outer to inner annulus, as does the biphasic permeability coefficient.

The protocol presented in chapter 5 is a first approach to gain insight in the separate influence of chemo-mechanical quantities on the mechanical behaviour of cartilaginous tissues. The division of the tissue fluid in two fluid compartments requires the definition of additional activity and osmotic coefficients. The fluid in the intrafibrillar compartment can as a fist approach be defined as having identical activity and osmotic coefficients as the external fluid, as was done in this study. The existing triphasic model, based on Donnan osmosis, needs to be improved, so that it is capable of fitting swelling transients with realistic diffusion coefficients. A start to this is the introduction of electro-chemical potentials for the cations and anions separately (quadriphasic theory). Subsequently, the concept of separation of the tissue water into two fluid compartments can be combined with the quadriphasic theory. 


\section{Samenvatting}

De oorzaken van lage rugpijn zijn in veel gevallen onduidelijk. Het is niet altijd mogelijk om een link te leggen tussen het ontstaan van lage rugpijn en het optreden van abnormaliteiten in de tussenwervelschijf. In het geval van een hernia kan soms een direct verband worden gevonden tussen de pijnsymptomen en de defecte tussenwervelschijf. Om te doorgronden hoe het mogelijk is dat bepaalde mechanische defecten aan de tussenwervelschijf optreden, is het nuttig om gebruik te maken van chemo-mechanische micromodellen van de tussenwervelschijf. De tussenwervelschijf draagt zijn mechanische belasting niet alleen via elastische en viskeuze krachten, maar osmotische, of electrochemische krachten spelen hierbij een belangrijke rol. Voor een goed begrip van de etiologie van tussenwervelschijfziekten en het proces van degeneratie van de tussenwervelschijf, is het van belang de bijdragen van deze drie krachten te kunnen scheiden. Op deze manier kan bijvoorbeeld een goede inschatting gemaakt worden van de spanning die optreedt in de vaste stof, wat zeer waarschijnlijk een belangrijke rol speelt bij hernia.

In het kader van het bovenstaande, heeft het onderzoek beschreven in dit proefschrift zich gericht op de ontwikkeling van (1) een numeriek model dat de elastische, osmotische en viskeuze krachten scheidt en (2) een experimenteel protocol, waarmee de bijdragen van de verschillende krachten bepaald kunnen worden. Het is mogelijk om met het protocol schattingen te bepalen van in vivo waarden van modelparameters, zoals elastische spanning, rek, activiteits- en osmotische coëfficiënten. Dit maakt het een nieuw waardevol instrument voor het testen van mengselmodellen van de tussenwervelschijf.

Het numerieke model is gebaseerd op de mengseltheorie, waarbij onderscheid gemaakt wordt tussen drie componenten (fasen): vaste stof, vloeistof of water en zoutmoleculen (ionen). Het kiezen van de vrijheidsgraden is gebaseerd op de vereiste, dat discontinuiteiten in de ionenconcentraties of gebonden ladingsdichtheid, niet leiden tot discontinuiteiten in de vrijheidsgraden. Hiermee is de continuiteit van de vrijheidsgraden aan de grenzen van het materiaal verzekerd.

De drie fasen mengseltheorie wordt beschreven in hoofdstuk 2. De interactie tussen de drie componenten is onderworpen aan de fundamentele behoudswetten van de fysica. Voor het aflejden van de differentiaalvergelijkingen van het mengsel worden de eerste (behoud van energie) en tweede (toename van entropie) hoofdwet van de thermodynamica gebruikt, waarbij de toename van entropie van toepassing is op het totale mengsel, niet op de afzonderlijke componenten. Als model voor het zwellen van het materiaal wordt Donnan osmose gebruikt. De belangrijkste aannames t.a.v. het model zijn: de componenten zijn intrinsiek incompressibel; de absolute temperatuur is constant in tijd en plaats; de volumefractie van de ionen wordt verwaarloosd in vergelijking met de volumefracties van de vaste stof en vloeistof; overdracht van massa en impulsmoment van de ene component op de andere wordt verwaarloosd; traagheids- en lichaamskrachten in de impulsvergelijking worden verwaarloosd.

Uit het drie fasen mengselmodel resulteren drie gekoppelde differentiaalvergelijkingen, die de beweging van de vaste stof, vloeistof en ionen beschrijven. Deze differentiaalvergelijkingen kunnen niet analytisch opgelost worden. Voor het oplossen van de vergelijkingen is derhalve gekozen voor een eindige-elementenmodel. De vrijheidsgraden zijn de verplaatsingsvector van de vaste st of en de chemische potentialen van de vloeistof en de zoutmoleculen (hoofdstuk 3). Voor de simulatie van een-dimensionale zwel- en 
consolidatie-experimenten is een een-dimensionaal computermodel ontwikkeld. In een zwel- en consolidatie-experiment wordt de hoogte van een proefstuk in de tijd gemeten als functie van de mechanische en chemische (osmotische) belasting. Het proefstuk zit opgesloten in een cylinder en is via een filter aan de onderkant in aanraking met een zoutoplossing (chemische belasting). Via een zuiger, die op het proefstuk rust, wordt het proefstuk mechanisch belast. Uit de computerfits van de zwel- en consolidatie-experimenten worden chemo-mechanische parameters, zoals activiteitscoëfficiënten, permeabiliteits- en diffusiecoëfficiënt, effectieve spanning e.l spanningsvrije lengte bepaald.

De evenwichtssituaties en de consolidatietransiënt van de experimenten kunnen makkelijk gefit worden. Het blijkt echter niet mogelijk om de zweltransiënt gelijktijdig met de consolidatietransiënt te fitten. Het blijkt ook dat de diffusiecoëfficiënt overschat wordt, terwijl de rest van de parameterwaarden zich in het fysiologische bereik bevinden.

De radiale variatie van de permeabiliteit in de annulus fibrosus van de tussenwervelschijf is onderzocht in hoofdstuk 4. De consolidatietransiënt van zwel-en consolidatie-experimenten is gefit met een analytische twee-fasen oplossing. Uit die oplossing zijn de twee-fasen permeabiliteitscoëfficiënt en de compressiemodulus (aggregate modulus) bepaald. De permeabiliteitscoëfficiënt neemt toe in radiale richting van buiten naar binnen. Dit resultaat wordt versterkt door de resultaten uit hoofdstuk 5, waar hetzelfde gevonden wordt. Voor de compressiemodulus spreken de resultaten van hoofdstuk 4 en 5 elkaar tegen. De resultaten van hoofdstuk 4 geven een toename van binnen naar buiten, terwijl uit de resultaten van hoofdstuk 5 het tegenovergestelde volgt. De twee-fasen permeabiliteit en de compressiemodulus zijn afhankelijk van de vervorming.

In hoofdstuk 5 zijn een serie experimenten gedaan om de in vivo chemo-mechanische eigenschappen van de annulus van tussenwervelschijf van de hond te bepalen. Uitgangspunten in het protocol voor de bepaling van deze eigenschappen zijn de opdeling van de vloeistof in extra- en intrafibrillair water (dubbel vloeistof compartiment) en het gebruik van de theorie van Donnan voor de berekening van de osmotische druk. De experimenten die aan de basis van het protocol liggen zijn: een-dimensionale zwel- en consolidatie-experimenten, meting van gebonden ladingen m.b.v. radioactieve labelling, meting van hydroxyproline en hoeveelheid vloeistof. Onderzoek heeft aangetoond, dat een deel van het weefselwater gebonden is aan de collageen fibrillen. Deze fractie water is niet toegankelijk voor de proteoglycanen en wordt de intrafibrillaire fractie genoemd. De grootte (hoeveelheid vloeistof) van het intrafibrillaire compartiment wordt bepaald door het collageen (hydroxyproline) gehalte en de asmotische drukken.

Uit de evenwichtssituaties van de zwel- en consolidatie-experimenten is een relatie tussen de effectieve spanning (een maat voor de vaste stof spanning) en de rek bepaald. Daarnaast zijn in vivo waarden van chemo-mechanische grootheden, zoals gebonden ladingsdichtheid, vloeistoffractie, collageen gehalte, osmotische en activiteitscoëfliciënten, effectieve spanning en spanningsvrije lengte, bepaald. Uit de consolidatietransiënten zijn waarden voor de twee- en drie-fasen permeabiliteitscoëficiënten bepaald. Voor de twee-fasen permeabiliteitscoëfficiënt is de twee-fasen analytische oplossing uit hoofdstuk 4 gebruikt, voor de drie-fasen permeabiliteitscoëfficiënt de een-dimensionale computersimulatie uit hoofdstuk 3. Voor de drie-fasen permeabiliteitscoëfficiënt is ook een schatting voor de in vivo waarde gemaakt. De computersimulatie is tevens gebruikt om andere chemo-mechanische parameters voor een enkel vloeistofcompartiment te bepalen, als in hoofdstuk 3. In tegenstelling tot het analyseprotocol voor de evenwichtssituaties, maakt 
het computermodel geen onderscheid tussen extra- en intrafibrillair water.

$\mathrm{Bij}$ de bepaling van de relatie tussen spanning en rek is gekozen voor een lineair verband tussen de effectieve 2de Piola-Kirchhoff spanning en de Green-Lagrange rek. De keuze van het spannings-rekverband heeft een belangrijke invloed op de spanning en rek, die berekend worden voor de in vivo situatie. In het geval van een lineaire relatie, blijkt dat in de in vivo situatie de vaste stof onder compressie is in de radiale richting. De opdeling van vloeistof in twee (extra- en intrafibrillaire) vloeistofcompartimenten, levert verschillende resultaten op in vergelijking met de resultaten op basis van een enkel vloeistofcompartiment (computersimulatie). De grootte van het verschil is afhankelijk van de hoeveelheid water, die gebonden is aan de collageenfibrillen. De analyse van de resultaten geeft geen aanleiding tot de introductie van een chemische expansie spanning voor het zwellen van het materiaal. Evenals de twee-fasen permeabiliteit, neemt de driefasen permeabiliteit in radiale richting in de annulus toe van buiten naar binnen.

Het protocol dat gepresenteerd is in hoofdstuk 5 is een eerste aanzet om inzicht. te krijgen in de gescheiden invloed van chemo-mechanische grootheden op het mechanisch gedrag van kraakbeenachtige weefsels. De opdeling van de weefselvloeistof in twee vloeistofcompartimenten vereist de definitie van additionele activiteits- en osmotische coëfficiënten. De vloeistof in het intrafibrillaire compartiment kan als eerste benadering met identieke activiteits- en osmotische coëfficiënten in vergelijking met de externe vloeistof gedefinieerd worden. Deze benadering is in dit proefschrift gebezigd. Het bestaande drie-fasen model, op basis van Donnan osmose, moet verbeterd worden, zodat het in staat is om zweltransiënten te fitten met realistische diffusiecoëfficiënten. Een aanzet hiertoe is de definitie van electrochemische potentialen voor de kationen en anionen afzonderlijk (vier-fasen theorie). De vier-fasen theorie kan vervolgens met het concept van de scheiding van het weefselwater in twee vloeistofcompartimenten uitgebreid worden. 


\section{Nawoord}

Met het grote risico mensen over het hoofd te zien (en dat kunnen er heel wat zijn in een periode van 5 jaar), wil ik dit nawoord gebruiken om een aantal mensen te bedanken, voor hun directe of indirecte bijdrage aan de totstandkoming van dit proefschrift. Het is niet de bedoeling om een volgorde van belangrijkheid te volgen. Als je jouw naam in dit stukje hoopt te vinden, dan kan dat dus overal zijn, of niet, in het geval ik het ben vergeten, of omdat je simpelweg de drempel niet gehaald heb.

Met een aantal collega's bij BW heb ik mij in de beginperiode prima kunnen vermaken middels de vele fietstochtjes in het voorjaar en in de zomer. Een aantal hebben indertijd vooral kennis gemaakt met mijn achterkant, gezien mijn neiging om voorop te willen sleuren; Harold en Maarten: bedankt dat jullie mij zo tactisch voorop hebben laten rijden (of ging het toch gewoon te hard?). Hans Snijders bezit helaas ook 'sleur-genen', maar die heeft me op een andere manier een stuk vooruit geholpen. Hans, bedankt voor al het voorwerk wat je hebt verricht. Mede daardoor is dit project uiteindelijk op een geslaagde manier afgerond. O.a. wat betreft het Unix-gebeuren wil ik Josv met name bedanken. Jos en ik hebben heel wat uurtjes samen gespendeerd in het 'SUN-hok'. Jos was nooit te beroerd om te helpen bij een praktisch probleem of te discussiëren over een wetenschappelijke vraag.

Nadat Harold en ik het ruige AIO-leven een beetje voor gezien hadden, hebben we ons nog jaren kunnen vermaken met onze 'nibble' manie. Insiders weten waar het hier om gaat (AIO-vijand nummer 1). Harold, ik wil je vooral bedanken voor je luisterend oor in sommige zware tijden, en de gezelligheid, die we in ons konijnenhok en daarbuiten hebben mogen meemaken. Met Maarten heb ik vele gezellige etentjes gehad, waar de hartige taart tot een synoniern voor eten met Maarten werd.

Voor de dagelijkse begeleiding van mijn project heeft in eerste instantie Maarten directe verantwoordelijkheid gedragen, daarna heeft Jacques dat overgenomen. Ik wil jullie allebei bedanken voor jullie bijdrage in het onderzoek. Jacques heeft voornamelijk het schrijfwerk gesuperviseerd en heeft dat voortreffelijk gedaan. Zijn suggesties en inzichten hebben zeker bijgedragen tot een verhoging van het nivo van het proefschrift en wat schrijven betreft heb ik veel van hem geleerd.

Voor het experimentele werk heb ik veel te danken gehad aan Paul Willems, die de opstelling heeft ontworpen en mij heeft bijgestaan bij het opstarten van de experimenten en het verwerken van de data. Gerrit van Kranenburg had gelukkig al een protocol voor hydroxyproline bepaling klaarliggen en heeft me met raad en daad in het biochemisch lab bijgestaan. Harry, bedankt voor het met raad en daad bijstaan bij mijn soft- en hardwareproblemen.

Andere AIO's, tot dusver nog niet vernoemd, hebben bijgedragen tot de gezelligheid op de vakgroep en daarbuiten. Bij Hennie heb ik een aantal gezellige etentjes met meestal stimulerende, soms tot droevig nivo zakkende conversatie meegemaakt. Jons, alhoewel geen lid van de vakgroep, toch een beetje kind aan huis bij $\mathrm{BW}$ heb ik vooral buiten de Uni beter leren kennen. Jons, het was een plezier om samen te discussiëren, sporten en er gewoon te zijn, zeker niet minder nadat Syl en Gebbe in jouw leven kwamen.

Op de vakgroep liepen soortgenoten rond met een (bijna) immer positieve instelling: Matthijs, René, Lars. Jongens, keep on smiling!

De mensen die mij geholpen hebben bij het voorbereiden en uitvoeren van de experi- 
menten wil ik bedanken. Eugène Raatschilders van anatomie, Chris Hannen, Huib Jussen en Jan Aerts van de mechanische werkplaats, Jos Kop van het Radionuclidenlab, Arjan Frijns als stagiair bij Jacques Huyghe.

Mijn promotores, Jan Janssen en Toon Huson hebben ieder op hun eigen manier hun invloed doen gelden op het proefschrift. Jan heeft de 'lijn' van het onderzoek in de gaten gehouden en op bepaalde momenten nuttig bijstuurwerk verricht. Toon wil ik vooral bedanken voor zijn steun en begrip in moeilijke tijden.

In de afgelopen 5 jaar zijn een aantal stagiaires de revue gepasseerd. Dank voor jullie bijdrage aan het onderzoek. Alhoewel de resultaten van het stagewerk niet direct zijn gebruikt voor dit boekje, is er toch waardevol werk verricht. (Gedeeltelijk) onder mijn supervisie hebben Paul Meurs, Marcel Deurhof, Ralph Graven en Arjan Frijns hun bijdrage verricht aan het tussenwervelschijfonderzoek.

Mijn acrobatiekclub heeft een belangrijke rol gespeeld in mijn leven vanaf het moment dat ik begonnen ben met het promotieonderzoek. Beste acrobatiekvrienden en -vriendinnen, heel hartelijk bedankt dat ik jullie al die jaren heb mogen tillen (snoekje, plankje, bolkje) en voor die paar momenten die ik als boven heb mogen meemaken.

Last but not least bedank ik mijn ouders en broer en schoonzus voor hun steun door de jaren heen, en Ingrid, voor jouw positieve invloed het laatste half jaar. 


\section{Curriculum Vitae}

Gerard Boudewijn Houben werd geboren op 19 oktober 1961 te Zaandam. Na een aantal omzwervingen (4 lagere scholen) doorliep hij het Gymnasium B op het Jansenius College te Hulst (1974-1980). In augustus 1980 toog hij naar Eindhoven om aldaar aan de TH Technische Natuurkunde te studeren. Deze studie werd in juni 1988 afgerond toen de TH inmiddels TU heette. Voor het afstudeerwerk (het meten van vloeistofdrukken in biologische weefsels) bij de vakgroep Biofysica van de Geneeskunde Faculteit van de RL was hij naar Maastricht verhuisd. Na het afstuderen volgde een periode van vervangende dienst. bij de vakgroep $\mathrm{K}$ (eel) N(eus) en O(orheelkunde), alwaar onderzoek naar het meten van stembandtrillingen gedaan werd (1989-90). Van 1991 tot 1996 is Gerard bezig geweest met het onderzoek op de Rijksuniversiteit Limburg, Faculteit der Gezondheidswetenschappen, Vakgroep Bewegingswetenschappen, dat leidde tot dit proefschrift. 

